\title{
Direct and Inverse Kinematics of a New Class of Serial-Parallel Hybrid Manipulator
}

\author{
by \\ James D. Robinson

\begin{abstract}
A Thesis submitted to
the Faculty of Graduate Studies and Research

in partial fulfilment of

the requirements for the degree of
\end{abstract} \\ Master of Applied Science \\ Ottawa-Carleton Institute for \\ Mechanical and Aerospace Engineering \\ Department of Mechanical and Aerospace Engineering \\ Carleton University \\ Ottawa, Ontario, Canada
}

January 2008

Copyright (C)

2008 - James D. Robinson 


$\begin{array}{ll}\begin{array}{l}\text { Library and } \\ \text { Archives Canada }\end{array} & \begin{array}{l}\text { Bibliothèque et } \\ \text { Archives Canada }\end{array} \\ \begin{array}{l}\text { Published Heritage } \\ \text { Branch }\end{array} & \begin{array}{l}\text { Direction du } \\ \text { Patrimoine de l'édition }\end{array} \\ \begin{array}{l}\text { 395 Wellington Street } \\ \text { Ottawa ON K1A 0N4 }\end{array} & \begin{array}{l}\text { 395, rue Wellington } \\ \text { Ottawa ON K1A ON4 }\end{array} \\ \text { Canada } & \begin{array}{l}\text { Canada } \\ \end{array}\end{array}$

Yourfile Votre référence

ISBN: 978-0-494-36832-9

Our file Notre référence

ISBN: 978-0-494-36832-9

NOTICE:

The author has granted a nonexclusive license allowing Library and Archives Canada to reproduce, publish, archive, preserve, conserve, communicate to the public by telecommunication or on the Internet, loan, distribute and sell theses worldwide, for commercial or noncommercial purposes, in microform, paper, electronic and/or any other formats.

The author retains copyright ownership and moral rights in this thesis. Neither the thesis nor substantial extracts from it may be printed or otherwise reproduced without the author's permission.
AVIS:

L'auteur a accordé une licence non exclusive permettant à la Bibliothèque et Archives Canada de reproduire, publier, archiver, sauvegarder, conserver, transmettre au public par télécommunication ou par l'Internet, prêter, distribuer et vendre des thèses partout dans le monde, à des fins commerciales ou autres, sur support microforme, papier, électronique et/ou autres formats.

L'auteur conserve la propriété du droit d'auteur et des droits moraux qui protège cette thèse. $\mathrm{Ni}$ la thèse ni des extraits substantiels de celle-ci ne doivent être imprimés ou autrement reproduits sans son autorisation.
In compliance with the Canadian

Privacy Act some supporting forms may have been removed from this thesis.

While these forms may be included in the document page count, their removal does not represent any loss of content from the thesis.
Conformément à la loi canadienne sur la protection de la vie privée, quelques formulaires secondaires ont été enlevés de cette thèse.

Bien que ces formulaires aient inclus dans la pagination, il n'y aura aucun contenu manquant.

\section{Canadä}




\section{Abstract}

Increasing the stiffness-to-weight ratio of serial manipulators is important in applications where a stiff manipulator with a large workspace and low weight is required. Traditional six degree-of-freedom (DOF) serial manipulators often utilize six revolute joints (6R-manipulator) and possess a large workspace, however in order to make them stiff the addition of structural weight is required in both the joint and link structure. Six DOF parallel manipulators, such as the Stewart-Gough platform (SGP), are very rigid with a high stiffness-to-weight ratio but possess a significantly smaller workspace. Hybrid manipulators attempt to use the positive characteristics of both types of manipulators to produce a manipulator with a high stiffness-to-weight ratio and a large workspace. The Griffis-Duffy platform (GDP) is a special configuration of SGP that is, in most configurations, subject to self-motions. Self-motions occur when a manipulator can move without actuator input, a usually undesirable characteristic. In this thesis a special configuration of GDP possessing well defined one DOF self-motion is used as a kinematic pair, replacing the revolute joints of a $6 \mathrm{R}$-manipulator in an attempt to increase the stiffness of the manipulator without a significant increase in structural weight. The kinematic pair constructed using the GDP is called an A-pair and a manipulator utilizing $n$ A-pairs as joints is called an $n \mathrm{~A}$-manipulator.

Implementing a novel joint architecture requires an investigation of the manipulator kinematics. This thesis explores the direct and inverse kinematics problem of Amanipulators. It is shown that the direct kinematics of A-manipulators is no more complicated than that of R-manipulators while the inverse kinematics problem is more challenging. In this thesis an algorithm for solving the inverse kinematics problem, that is obtaining all sets of joint variables that place the end effector (EE) of the manipulator in a desired 
pose, using kinematic mapping is proposed. The algorithm, which is applied to 4A- and $6 \mathrm{~A}$-manipulators, theoretically breaks the manipulators into two sub-chains $(2 \mathrm{~A}$ - and $3 \mathrm{~A}$ chains respectively). For each new chain a technique called kinematic mapping is used to represent the displacements of the EE of each sub-chain as points in a seven dimensional projective space, $P^{7}$. The set of all points achievable by any chain is called the constraint variety. By intersecting the constraint varieties of the sub-chains in $P^{7}$, the solution of the inverse kinematics problem for the A-manipulator is obtained. 
This thesis is dedicated to my family and friends for all of their support. 


\section{Acknowledgments}

I would like to acknowledge several people who have provided help and support throughout my thesis work.

- I would like to start off by thanking my supervisor Prof. John Hayes for his advice and support throughout all of my thesis work. He provided me with an interesting topic to develop and provided advice, guidance and encouragement when I needed it. He also helped organized the trip of my lifetime to Austria and for that I am forever in his debt.

- Many thanks are also in order for Manfred Husty for his help via email and during my stay at the University of Innsbruck. His help was invaluable at every step and without his guidance I don't know if I would have been able to complete my research. I also appreciated his hospitality when he took me to his home and Semriach to experience great food, great wine and Wiesswurst.

- Everyone at Institute for Geometry and CAD at the University of Innsbruck, Austria. To Martin Pfurner and Hans-Peter Schröcker for their technical knowledge, their hospitality, introducing me to their families, taking me on hikes into the mountains, the ski trip on my birthday (which I will never forget) and helping me feel at home even though I was far from it. To Patrick Taschwer for sharing his office with me, taking me on ski trips, fixing my bike and, of course, introducing me to Fleischkaessemmel. To Carina Wibmer for helping me with life in Austria (and my trips to other cities) and to Dominic Walter for his help with my mathematics problems especially with Gröbner bases. 
- My friends from MAAE at Carleton for the creation of many things that made life a lot more interesting, including 3284ME and Beer O'clock, Coupling, Family Guy lunches, tennis, hockey and the $\pi \ln$ 's. This includes (by office blog nicknames, in no particular order) BIC, awall, Mittens, Pancake D., The Conquistador, PMW, The Polish Pickle, Daemon, Captain Obvious, Hooligan, Miss T., The guy with the accent and anyone else I may have forgotten. Also to those in my "research group": Pin, Mark, Andrew and Alexis.

- My friends at home and abroad, especially all of my many roommates over the years and my ultimate frisbee teams (Mayday Malones, Discrepencies, Ultimate4play). A Special thanks to Rob and Lynea for letting me stay in their new home.

- And finally to my family. Thank you to my parents Gwen and Don and my stepparents Andy and Karen for all their support over my many years at university. Also to my brothers Tom, Cody, Casey, Kevin and Craig. Without the support of my family I do not think I could have stayed in school this long. 


\section{Table of Contents}

$\begin{array}{ll}\text { Abstract } & \text { ii }\end{array}$

Acknowledgments $\quad$ v

Table of Contents $\quad$ vii

List of Tables $\quad$ xi

List of Figures $\quad$ xii

1 Introduction $\quad 1$

1.1 Problem Statement . . . . . . . . . . . . . . . 3

1.2 Organization of Thesis . . . . . . . . . . . . . . 3

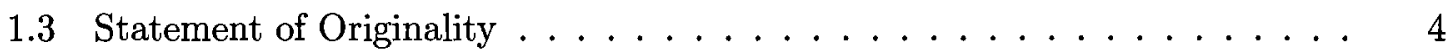

2 Required Background Theory $\quad 6$

2.1 Degree-of-Connectivity and Degrees-of-Freedom . . . . . . . . . . 6

2.2 Kinematic Pairs . . . . . . . . . . . . . . . . . 7

2.3 Serial, Parallel and Hybrid Manipulators . . . . . . . . . . . . . 10

2.3 .1 Serial Manipulators . . . . . . . . . . . . . 10

2.3.2 Parallel Manipulators . . . . . . . . . . . . . . . . 11

2.3.3 Hybrid Manipulators . . . . . . . . . . . . . . . 13

2.4 Representations of Spatial Displacements . . . . . . . . . . . . . . 14

2.4 .1 Homogeneous Coordinates . . . . . . . . . . . . . . . . 14 
2.4.2 Displacements in $E_{3} \ldots \ldots \ldots \ldots \ldots \ldots$

2.4 .3 Groups ............................... 16

2.4.4 Quaternions and Representation of Rotations in $E_{3} \ldots \ldots 17$

2.4.5 Dual Quaternions and Representing Displacements in $E_{3} \ldots \ldots$

2.4.6 Kinematic Mapping . . . . . . . . . . . . . . . . 22

2.4.7 Derivation of Study Parameters . . . . . . . . . . . . . 23

2.5 Denavit Hartenberg Parameters and Direct Kinematics . . . . . . . . 25

2.5.1 Assigning Reference Frames and DH-Parameters . . . . . . . . 25

2.5 .2 Direct Kinematics of $n$ R-Chains $\ldots \ldots \ldots \ldots$

2.6 Effect of Transformations in $E_{3}$ on Points in $P^{7} \ldots \ldots \ldots \ldots$

2.6.1 Transformation in the Base Frame . . . . . . . . . . . . . 29

2.6.2 Transformations in the Moving Frame . . . . . . . . . 30

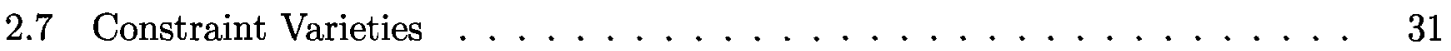

2.8 Tangent of the Half-angle Substitution $\ldots \ldots \ldots \ldots \ldots$

2.9 Resultant Elimination . . . . . . . . . . . . . . . . . . 34

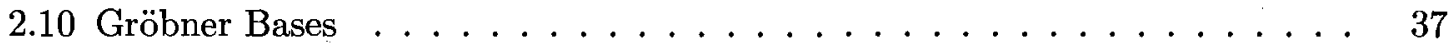

2.10 .1 Introduction to Gröbner Bases . . . . . . . . . . . . . . 38

2.10 .2 Obtaining the Gröbner Bases $\ldots \ldots \ldots \ldots$

2.11 Polynomial Homotopy and Continuation Methods . . . . . . . . . 46

2.11 .1 Introduction to Continuation Methods . . . . . . . . . . . . 46

2.11 .2 Selecting a Start System . . . . . . . . . . . . . . . 47

2.11 .3 Projective Transformation . . . . . . . . . . . . . . 49

2.11 .4 Path Following . . . . . . . . . . . . . . 50

\section{Review of Previous Research $\quad 52$}

3.1 Overview of Griffis-Duffy Platforms . . . . . . . . . . . . . . 52

3.1.1 Description of GDP and Possible Configurations . . . . . . . . 52

3.1 .2 Self-motions of GDPs $\ldots \ldots \ldots \ldots \ldots \ldots$

3.2 Inverse Kinematics of 6 R-Manipulators $\ldots \ldots \ldots \ldots \ldots$ 
3.2.1 Overview of Some Methods of Solving the Inverse Kinematics Problem 59

3.2.2 Inverse Kinematics of a 6R-manipulator Utilizing Kinematic Mapping 61

4 Introduction to A-Pairs and A-Chains 65

4.1 The A-Pair Using a GDP as a Kinematic Pair . . . . . . . . . . . . . . . 65

4.2 Setup of A-Chains . . . . . . . . . . . . . 68

4.2 .1 A-Chain Configuration . . . . . . . . . . . . . 69

4.2 .2 The DH-Parameters of an A-Chain . . . . . . . . . . 69

4.3 Direct Kinematics of A-Chains . . . . . . . . . . . . 71

4.4 Obtaining the Constraint Varieties of Canonical A-Chains . . . . . . . 73

4.4.1 Constraint Variety of a Single A-pair . . . . . . . . . . . 74

4.4.2 Constraint Variety of Canonical 2A-Chains . . . . . . . 76

5 Algorithm for the Inverse Kinematics of A-Chains $\quad 80$

5.1 Inverse Kinematics of a 4 A-Manipulator . . . . . . . . . . . . 81

5.1.1 Theory Behind 4A-manipulator Inverse Kinematic Algorithm . . . 81

5.1.2 Numeric Example of Inverse Kinematics for a 4A-manipulator . . 89

5.2 Inverse Kinematics of a 6 A-Manipulator $\ldots \ldots \ldots \ldots$

5.2.1 Theory Behind the 6A-manipulator Inverse Kinematic Algorithm . . 95

5.2.2 Numeric Example of Inverse Kinematics for a 6A-manipulator . . . 102

6 Conclusions and Future Research $\quad 109$

6.1 Concluding Remarks . . . . . . . . . . . . . . . . 109

6.2 Suggestions for Future Research . . . . . . . . . . . . . . 110

$\begin{array}{ll}\text { List of References } & 112\end{array}$

Appendix A Equations of Constraint Variety for a Canonical 2A-chain in

$\begin{array}{ll}\text { Terms of } u_{i+1} & 116\end{array}$

Appendix B Equations for 4A-manipulator Numeric Example 118

B.1 Left 2A-chain Constraint Variety . . . . . . . . . . . . 118 
B.2 Right 2A-chain Constraint Variety . . . . . . . . . . . . . 119

B.3 Joint Variables of Left 2A-chain . . . . . . . . . . . . . . . . . 121

B.4 Joint Variables of Right 2A-chain . . . . . . . . . . . . . 123

Appendix C Equations for 6A-manipulator Numeric Example 126

C.1 Left 3A-chain Constraint Variety . . . . . . . . . . . . . . 126

C.2 Right 3A-chain Constraint Variety . . . . . . . . . . . . 133 


\section{List of Tables}

3.1 Coordinates of anchor points for special midline-to-vertex configuration. . . 56

4.1 DH-parameters for 3A-manipulator direct kinematics numeric comparison. . 72

5.1 DH-parameters for 4A-manipulator inverse kinematics numeric example. . . 89

5.2 Target joint variables for 4A-manipulator inverse kinematics numeric example. 90

5.3 Comparison of target joint variables with algorithm results for 4Amanipulator inverse kinematics numeric example. . . . . . . . . . . . 94

5.4 DH-parameters for 6A-manipulator inverse kinematics numeric example. . . 102

5.5 Target joint variables for 6A-manipulator inverse kinematics numeric example.103

5.6 Comparison of target joint variables with one set of the resulting joint variables for 6A-manipulator inverse kinematics numeric example. . . . . . . . . 107 


\section{List of Figures}

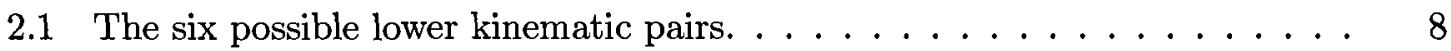

2.2 Example of a serial manipulator from KUKA Robotics. (www.kuka.com) . 11

2.3 Example of a parallel manipulator from CAE. (www.cae.com) . . . . . . . . 12

2.4 Sketch of a redundant hybrid manipulator from the Shenyang Institute for

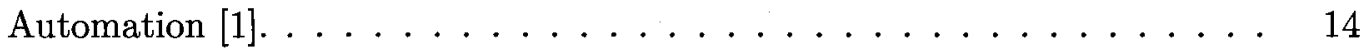

2.5 Link with reference frames and DH-parameters. . . . . . . . . . . . 27

2.6 Projection of the constraint of an arbitrary planar $2 \mathrm{R}$-chain to a three dimensional subspace of the kinematic mapping image space. . . . . . . . . . 32

3.1 Example of the vertex-to-vertex configuration of GDP. . . . . . . . . . . 54

3.2 Example of the midline-to-vertex configuration of GDP. . . . . . . . . 55

3.3 Midline-to-vertex GDP with coordinate systems and variables defined. . . . 57

3.4 Illustration of inverse kinematics problem for a general 6R-manipulator. . . 62

3.5 Illustration of left and right 3R-chains with reference frames. . . . . . . 62

4.1 Example application of A-pair jointed manipulator where the R-pair joints of the International Space Station robot arm (left) are replaced (right) with

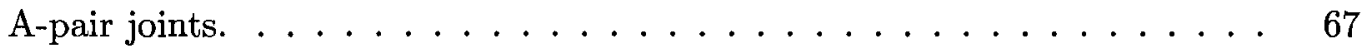

4.2 The DH-parameters applied to a link in an A-chain. . . . . . . . . . 70

4.3 Canonical 1A-pair with reference frames $\Sigma_{i-1}$ and $\Sigma_{i}^{\prime}$. . . . . . . 75

4.4 Canonical 2A-chain with reference frames $\Sigma_{i-1}, \Sigma_{i}^{\prime}, \Sigma_{i}$ and $\Sigma_{i+1}^{\prime} \ldots \ldots 77$

5.1 4A-manipulator showing the theoretical break between the left and right 2A-

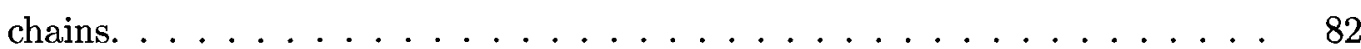

5.2 2A-pair illustrating the addition of the second link to the left chain with $\Sigma_{L} . \quad 84$ 
5.3 2A-pair illustrating the addition of the fourth link to the canonical $2 \mathrm{~A}$-chain in order to obtain the right chain with $\Sigma_{R}$ and the target frame $\Sigma_{E E}$ relative to the base frame $\Sigma_{0} \ldots \ldots \ldots \ldots \ldots \ldots$

5.4 6A-manipulator showing the theoretical break between the left and right 3A-

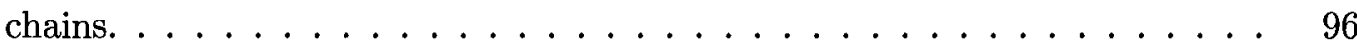

5.5 3A-pair illustrating the addition of the third joint and third link to the left chain with $\Sigma_{L} \ldots \ldots \ldots \ldots \ldots \ldots \ldots \ldots$

5.6 3A-pair illustrating the addition of joint 4 and link 4 to the left chain with $\Sigma_{L} .99$ 


\section{Chapter 1}

\section{Introduction}

Many applications of serial robotic manipulators require a lightweight construction. Perhaps the most high profile example of this is the Canadarm, used on the space shuttle and International Space Station. Even in terrestrial applications lower weight is a useful characteristic because smaller motors and less materials are required, thus reducing costs. The problem with simply reducing the weight of the components in an existing serial manipulator is that the overall stiffness is sacrificed.

Before continuing, it is important to understand the three main classes of manipulators and their characteristics with regards to workspace and stiffness-to-weight ratio. These classes of manipulators are discussed in more detail in Section 2.3 but are introduced here. The terms reachable workspace and dextrous workspace are used here and throughout this thesis. Reachable workspace refers to the volume of space in which the end effector (EE) of a manipulator (often referred to as the tool) can be placed in at least one orientation by actuating the joints of the manipulator. Dextrous workspace is a subspace of the reachable workspace and refers to the volume of space, if any, that the EE can be placed in any orientation.

Serial Manipulator: The EE of the manipulator is connected to the base by links which each connect to exactly two other links (with the exception of the base and EE links, which only connect to one other link). These connections are made by joints which are actuated to control the EE position. There is exactly one path between the EE 
and the base. This is what many consider to be a traditional robot arm, they are often constructed to resemble a human arm with shoulder, elbow and wrist joints. The reachable and dextrous workspace are often, but not always large, however a large amount of weight is often required to maintain stiffness, decreasing the stiffnessto-weight ratio.

Parallel Manipulator: The EE is connected to the base by multiple serial manipulators or legs. There are two or more paths between the EE and base. The legs are actuated to control the position of the end effector. The traditional flight simulator platform is a common example. The stiffness-to-weight ratio is increased relative to the serial manipulator by the many connections between the base and end effector since the load can be distributed through multiple paths. The workspace is greatly decreased by factors such as legs interfering with each other.

Hybrid Manipulator: A manipulator that is constructed by connecting parallel manipulators in series with other parallel or serial manipulators. A common configuration using multiple parallel platforms is where the EE of one parallel manipulator becomes the base of the next, and so on. By actuating the legs of each parallel manipulator the position of the EE can be controlled. This allows for a compromise of the increased work space of a serial manipulator with the stiffness of a parallel manipulator.

In many configurations of parallel manipulators the EE is controlled by actuating one or more of the joints in each leg, such as changing the length of the legs through a prismatic joint. In some special configurations the parallel manipulator is subject to self-motions, a situation where the EE can be moved without actuator input. In normal operations of a parallel manipulator this is an undesired trait, however this thesis proposes using such self-motions and the stiffness of parallel manipulators to possibly enhance the stiffness of serial manipulators, thus creating a special form of hybrid manipulator by replacing the revolute joints of a manipulator with a special configuration of a parallel manipulator that is subject to self-motions. It is not explicitly shown in this thesis that such an architecture results in a manipulator with a higher stiffness-to-weight ratio, however the effect on the 
manipulator kinematics of the substitution of the special parallel manipulator into a serial chain are explored.

\subsection{Problem Statement}

The purpose of this thesis is to examine the effect on the direct and inverse kinematics of replacing the revolute joints of a six revolute joint serial manipulator with a special configuration of parallel manipulator that is subject to self-motions. In this thesis the parallel manipulator being employed possesses self-motions characterized by a well-defined one degree-of-freedom (DOF) motion that couples rotation and translation. The hybrid manipulator presented utilizes this parallel manipulator as the joints of a serial chain.

The term direct kinematics refers to knowing the position and orientation, the pose, of the $\mathrm{EE}$ for a given set of joint variable values, while the term inverse kinematics refers to determining the required sets of joint variable values to achieve a desired EE pose.

The goal of this thesis is to show the development of novel algorithms for the direct and inverse kinematics of a hybrid manipulator using four and six kinematic pairs constructed using a specific configuration of a parallel manipulator that is subject to self-motions as joints between links of a serial chain. The configuration of this new class of hybrid manipulator will be discussed in detail within this thesis.

\subsection{Organization of Thesis}

This thesis begins with an overview of the background theory required to understand the new work presented. The first background theory sections present several topics dealing with kinematics, including a more detailed examination of serial, parallel and hybrid ma-

nipulators; the types of joints commonly used in these manipulators; a look at methods of defining displacements in space, focusing on kinematic mapping and the kinematic image space; the use of Denavit Hartenbeg notation to quantify the kinematic geometry of a serial manipulator; the effects of Euclidean transformations on the image space; and the concept 
of constraint varieties. This is followed by a look at algebraic and geometric concepts that are required to understand the contents of this thesis. This includes an overview of resultant elimination, Gröbner bases, and polynomial homotopy continuation methods.

The concepts of the background theory sections are used to review the existing research that motivated this thesis and on which this thesis topic is based. This includes an examination of the self-motions of a special configuration of parallel manipulator called the Griffis-Duffy platform, and some of the existing methods of solving the inverse kinematics of serial manipulators with six revolute pairs as joints.

The original contributions of this thesis begin with a definition of the construction of the hybrid manipulator built using a novel kinematic pair on which this thesis is based. The next sections describe how the topics of the background theory sections are used to obtain the direct kinematics equations and determine the constraint varieties for chains with increasing numbers of joints. These constraint varieties are then used to set up an algorithm to obtain the solution to the inverse kinematics problem for chains with four and six of the special joints. Numerical examples are presented for both the four and six jointed manipulators. The final section examines some remaining numerical issues with the inverse kinematics of the six-jointed chain.

The thesis concludes with a summary of the results and a discussion of potential future research.

\subsection{Statement of Originality}

To the best of the knowledge of the author of this thesis the following list identifies contributions presented in this thesis that stem from original ideas, or results.

1. The use of Griffis-Duffy platforms that are subject to self-motions as a kinematic pair (called A-pairs) in a serial chain (called an A-chain).

2. Obtaining the forward kinematics equations for a general $n \mathrm{~A}$-chain.

3. Constructing the sets of equations that describe the constraint varieties for a single 
A-pair, a 2A-chain and a 3A-chain.

4. An algorithm for obtaining the inverse kinematics of a 4A-manipulator using kinematic mapping.

5. The modifications to an algorithm for the inverse kinematics of a $6 \mathrm{R}$-manipulator using kinematic mapping presented by Pfurner [2] to make it applicable to a $6 \mathrm{~A}$ manipulator.

6. Examples of the inverse kinematics of a $4 \mathrm{~A}$-manipulator and a $6 \mathrm{~A}$-manipulator. 


\section{Chapter 2}

\section{Required Background Theory}

This chapter reviews the basic background theory required to understand the previous and new research presented in this thesis. The first sections focus on the mechanical systems and kinematics while the later sections look at the mathematical and numerical techniques required.

\subsection{Degree-of-Connectivity and Degrees-of-Freedom}

A kinematic chain is often defined, as by Shigley and Uicker [3], as a collection of rigid bodies (links) connected by mechanical constraints (joints) such that there is relative motion between links. The term degree-of-connectivity (DOC) of a link describes the number of other links joined to it.

The degree-of-freedom (DOF) of a kinematic chain is defined to be an integer value corresponding to the minimum number of independent coordinates required to fully describe, geometrically, an arbitrary configuration of the chain. A kinematic chain constitutes a mechanism if its DOF is a positive value; a statically determinant structure if the DOF is zero; and a hyper-static (statically under-determined, or over-constrained) structure if the DOF is a negative value. An unconstrained rigid body in 3-dimensional space would have six DOF (three translational DOF and three rotational DOF). Each constraint applied by a joint reduces the DOF by one.

A typical, yet occasionally misleading, method for obtaining the DOF of a mechanical 
system uses the Chebyshev-Gubler-Kutzbach method which is discussed by Brunnthaler [4] and Pfurner [2]. In this method the the DOF is given by the formula

$$
\mathrm{DOF}=d(l-1)-m-\sum_{i=1}^{j} \mu_{i}
$$

where $d$ is the maximum DOF in the working space ( $d=3$ for planar or $d=6$ for spatial), $l$ is the number of links including the fixed base, $j$ is the number of joints, $\mu_{i}$ is the number of constraints imposed by joint $i$ (different joint types are discussed in section 2.2) and $m$ is the number of idle DOF in the chain. An idle DOF is one that does not affect the transmission of motion in a chain, such as a link with spherical joints at either end such that it can spin freely about the line connecting the two spherical joints. Some authors do not include $m$ in this analysis because to fully represent the manipulator even the idle DOF are required.

Equation (2.1) must be used carefully since in some mechanisms, such as the Bennett mechanism, which is described by Brunnthaler in [5] and [4], the equation produces misleading results. The Bennet mechanism is a closed spatial kinematic chain consisting of four links connected by four revolute joints with joint axes that are all skew relative to each other. Equation (2.1) suggests that this is a hyper-static rigid structure with DOF $=-2$. Despite this, the Bennet mechanism possesses one DOF.

\subsection{Kinematic Pairs}

Within the study of kinematics the joint between two links in a mechanism is usually classified as either a higher or lower kinematic pair. The term kinematic pair, or just pair refers to the two different links somehow being connected at a joint. Higher pairs are generally instances where two links meet with point, line or curve contact. Examples of these are cam and followers, spur gears or a rack and pinion. Higher pairs are not pertinent to this thesis. Lower pairs are characterized by surface contact between the two links. There are six possible lower pair joints. Each joint type has $n$ DOF, where $n$ describes 
the number of parameters needed to fully describe the state of the joint, the number of constraints applied by the joint is equal to $6-n$ in three-dimensional space, or $3-n$ in the plane. Figure 2.1 illustrates the six possible lower kinematic pairs.

Revolute

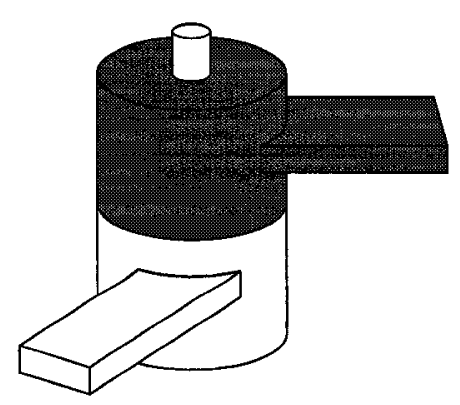

Cylindrical

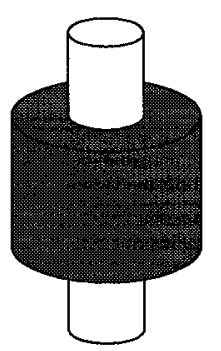

Helical

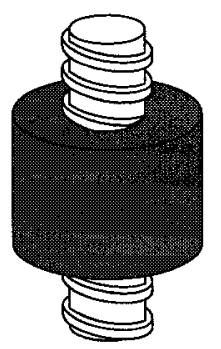

\section{Prismatic}

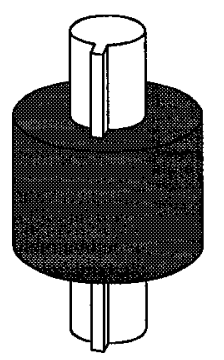

Spherical

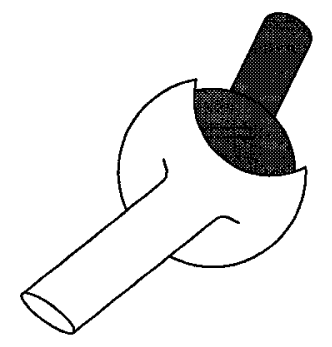

Planar

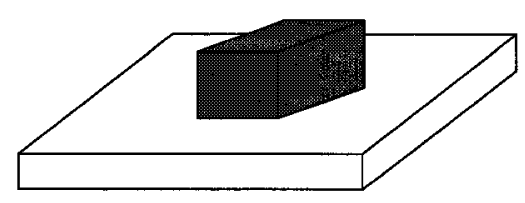

Figure 2.1: The six possible lower kinematic pairs.

Revolute: (R-pair) A basic hinge where the angle of one link relative to the other can be controlled. The R-pair has $1 \mathrm{DOF}$, the angle of rotation about the joint axis.

Prismatic: (P-pair) One link can slide or translate relative to the other. Such as a piston 
with a keyway or other non-cylindrical cross-section that prevents rotation. The Ppair has $1 \mathrm{DOF}$, the translation along the joint line-of-action (often erroneously called the "joint axis").

Cylindrical: (C-pair) One link can translate and rotate relative to the other. Examples include cylindrical pistons such as bicycle air pumps, or other mechanisms with two concentric cylinders. The C-pair has 2 DOF, rotation about common axis of cylinders and translation along the line of the axis.

Spherical: (S-pair) Commonly called a ball-joint. A sphere is surrounded by a concentric 'shell' sphere. The S-pair has 3 DOF, rotation about the three intersecting orthogonal axes.

Helical: (H-pair) Commonly called a screw-pair. Two mated helical surfaces move relative to each other such that one link has a coupled translation and rotation relative to the other. A screw with pitch $S$ translates $S$ units with each complete rotation of the joint. A common example is a matching nut and bolt. The H-pair has 1 DOF, either the translation along the joint line-of-action or rotation angle about the centreline of the helix fully describes the joint because they are related by $S$.

Planar: (E-pair) Links contact at a common plane and can slide relative to each other without lifting. Any planar object (eg. a book) sliding on a table is an example of an E-pair. The E-pair has 3 DOF, translation in the $x$ - and $y$-directions and rotation about an axis parallel to the $z$-axis, where the $z$-direction is the normal to the common plane. The ' $E$ ' label comes from embene, the German word for plane.

A detailed description of lower pairs is given by Hunt in [6]. Many more complex joints may be represented by two or more of the six basic lower pairs, such as a Universal-joint (U-joint) which may be represented by two R-pairs with intersecting axes. An S-pair may also be simplified by representing it as three R-pairs with mutually orthogonal intersecting axes. Understanding the characteristics of lower pairs is important to the kinematic analysis of robotic manipulators. 


\subsection{Serial, Parallel and Hybrid Manipulators}

This section examines in more detail the serial, parallel and hybrid classes of manipulators. Focus is placed on the defining features and nomenclature, examples of common configurations and characteristics with regards to workspace, stiffness to weight ratio and kinematic control.

\subsubsection{Serial Manipulators}

A serial manipulator can be described as an open chain where each link is connected to exactly two others $(\mathrm{DOC}=2)$, with the exception of the $\mathrm{EE}$ which is connected to only one other link $(\mathrm{DOC}=1)$. The common method of connecting the links is using lower pair joints, and more specifically R- and P-pairs, though others may be used. A serial manipulator consisting of links connected by $n \mathrm{R}$-pairs is referred to as an $n \mathrm{R}$-manipulator. Similar naming conventions can be used for the other joint types, or a combination of different types. The most well known serial manipulators are industrial robot arms. An example of a serial manipulator is shown in Figure 2.2.

In general, a $6 \mathrm{R}$-manipulator has $6 \mathrm{DOF}$ at the $\mathrm{EE}$ in most configurations. That means that in certain positions in space it can achieve any orientation. This set of positions is its dextrous workspace. Because serial manipulators have only one connection to the base or ground, in general the dextrous workspace, if it exists, is large compared to their parallel counterpart. It is important to note that not all 6R-manipulators posses a dexterous workspace, it is dependant on the joint and link geometry. This single connection to the base is also a disadvantage because the manipulator essentially becomes a cantilever beam, meaning stiffness is lower than if there were more connections with the base. To increase the stiffness of such a manipulator weight is added in the form of stiffer links and joints, leading to a low stiffness-to-weight ratio.

As will be shown later in this thesis, obtaining the direct kinematics for serial manipulators has a well known and simple solution, but the inverse kinematics problem is not 


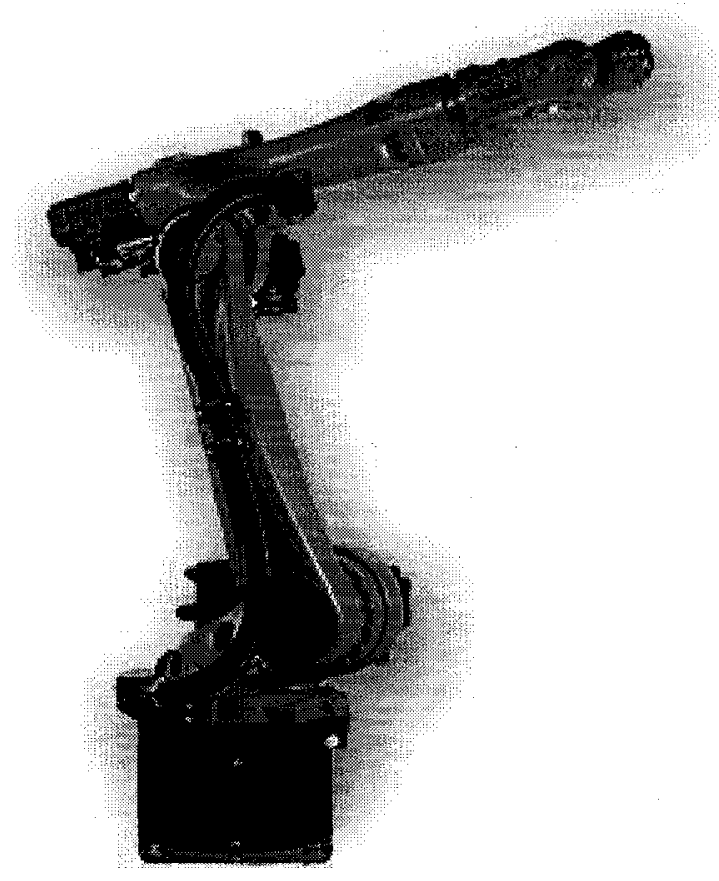

Figure 2.2: Example of a serial manipulator from KUKA Robotics. (www.kuka.com)

as trivial, requiring a complex algorithm to obtain the solution, and for some serial manipulators with lower pair joints it is still an open problem. The inverse kinematics of 6R-manipulators is addressed in more detail in Section 3.2.

\subsubsection{Parallel Manipulators}

A parallel manipulator is characterized by having two or more serial manipulators working simultaneously on the EE. The serial chains work together to influence the motion of the EE.

There exist many configurations of parallel manipulator, however the most relevent to this thesis is the Stewart-Gough Platform (SGP). In this configuration six legs connect the fixed base to the moving EE platform, the fixed base and moving platform have $\mathrm{DOC}=6$. The legs are often SPS, UPU, SPU or UPS configuration or a combination of S and U 
joints surrounding a $\mathrm{P}$ joint. Parallel manipulators are covered in detail by Merlet [7]. The moving platform is controlled by independently actuating the $\mathrm{P}$ joints in each of the legs. The multiple legs connecting the moving EE platform to the fixed base means that the parallel manipulator is inherently more stiff than a serial chain since the EE is no longer at the end of a cantilevered arm. This leads to the parallel manipulator having a higher stiffness-to-weight ratio than its serial counterpart as is discussed by Carbone and Ceccarelli [8]. The additional legs also have a negative aspect, they lead to self-collisions where the legs interfere with each other and the rest of the manipulator structure, this means there is a restricted reachable workspace and often no dextrous workspace at all. Figure 2.3 shows a flight simulator from CAE. The motion platform for the simulator is a Stewart-Gough platform.

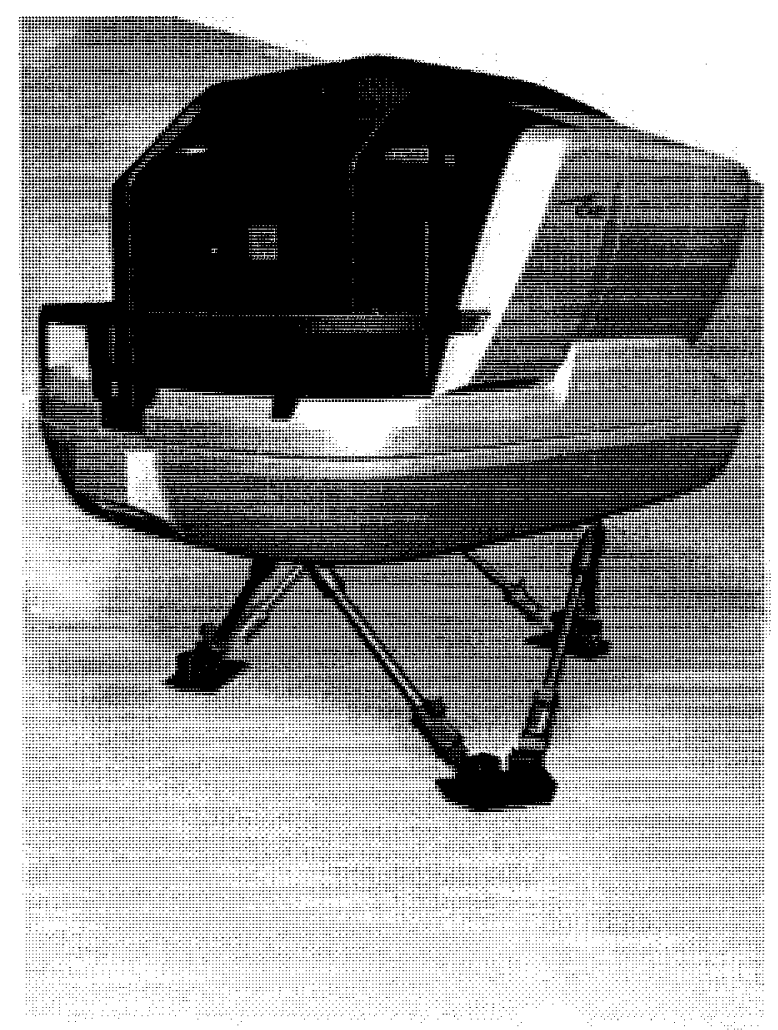

Figure 2.3: Example of a parallel manipulator from CAE. (www.cae.com)

The inverse kinematics of a parallel manipulator provides a simple unique solution for the leg lengths for every desired EE pose. On the other hand, the direct kinematics for a general 
SGP were an unsolved problem until a solution was provided by Husty [9]. Husty's algorithm for the direct kinematics of general SGPs obtains a $40^{\text {th }}$-order univariate polynomial that is solved to obtain the EE pose for given joint parameters. This means that in general, for each set of six leg lengths there are up to 40 possible EE poses.

\subsubsection{Hybrid Manipulators}

Hybrid manipulators are typically characterised by combining parallel and serial manipulators or concatenating parallel manipulators such that the moving platform of one parallel manipulator becomes the fixed base of the next, creating a serial chain of parallel manipulators. The intent of hybrid manipulators is to take advantage of the high stiffness of parallel manipulators and the typically larger workspace of serial manipulators.

One common example of a hybrid architecture is to concatenate two parallel manipulators with 3 DOF such that the moving platform of the second platform has 6 DOF relative to the fixed base of the first manipulator. Such manipulators are presented by Zheng, Bin and Luo [10] and Romdhane [11]. Another hybrid architecture involves attaching a serial manipulator onto the moving platform of a parallel manipulator. An example of this is given by Carbone and Ceccarelli [8] where a 2 DOF serial manipulator is attached to the moving platform of a 3 DOF parallel manipulator. Zhao et al. [1] and Ibrahim and Khalil [12] discuss the kinematics and dynamics of hybrid manipulators constructed by assembling any number of 3 DOF parallel manipulators in series resulting in redundant manipulators. The term redundant refers to the fact that for many poses in the workspace there is an infinite number of joint configurations that achieve the pose or that there are more actuated joints than DOF. Figure 2.4 shows a sketch of a redundant hybrid manipulator from the Shenyang Institute for Automation [1].

Each new configuration of hybrid manipulator has a different kinematics and control scheme. The kinematics must be re-evaluated for each new architecture. 


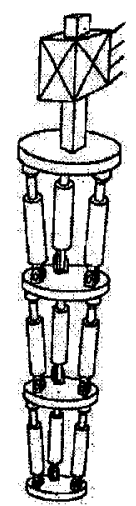

Figure 2.4: Sketch of a redundant hybrid manipulator from the Shenyang Institute for Automation [1].

\subsection{Representations of Spatial Displacements}

Critical to understanding the work of this thesis is understanding how displacements in three dimensional Euclidean space can be represented as points in a seven dimensional projective geometric space. This section introduces the tools and concepts required to work with this representation.

\subsubsection{Homogeneous Coordinates}

A cartesian coordinate system with origin $O$ and orthogonal axes $x, y, z$ is established in Euclidean space, $E_{3}$. A point $S$ in this space, and the ray passing through $O$ and $S$ are described by the cartesian coordinates $(x, y, z)$. If another distinct point $Q$ is selected on this ray away from the origin, it can be represented by $(\mu x, \mu y, \mu z)$. With this representation if $\mu \rightarrow \infty$, then $Q$ becomes the seemingly meaningless triple $(\infty, \infty, \infty)$. If homogenous coordinates are introduced, some meaning can be brought to the point at infinity. A homogenizing coordinate is introduced such that

$$
x=\frac{x_{1}}{x_{0}}, \quad y=\frac{x_{2}}{x_{0}}, \quad z=\frac{x_{3}}{x_{0}},
$$

where $x_{0} \neq 0$. The homogenous coordinates of $S$ are now written as the ratios $\left(x_{0}: x_{1}\right.$ : $\left.x_{2}: x_{3}\right)$. Note that the European convention has been used here, that is the homogenizing 
coordinate $x_{0}$ is the first element of the coordinate group, as opposed to the North American notation that uses $\left(x_{1}: x_{2}: x_{3}: x_{4}\right)$, where $x_{4}$ is the homogenizing coordinate. The European convention will be used throughout this thesis because by assigning $x_{0}$ as the homogenizing coordinate means that points in any $n$ dimensional projective space will have the homogenizing coordinate in the first position. An example of use of the European convention is Pfurner [2], and an example of the North American convention is Hayes, Zsombor-Murray and Chen [13].

In this representation if $x_{0}=1$, then the original cartesian coordinates of $S$ are recovered. If two proportional sets of homogenous coordinates are given, i.e. $\left(x_{0}: x_{1}: x_{2}: x_{3}\right)$ and $\left(\lambda x_{0}: \lambda x_{1}: \lambda x_{2}: \lambda x_{3}\right)$, where $\lambda \neq 0$, they represent the same point in space. If $x_{0} \rightarrow 0$, this is similar to when $\mu \rightarrow \infty$ in cartesian coordinates, the difference now is that $\left(0: x_{1}: x_{2}: x_{3}\right)$ describes the point at infinity on the line $O S$.

\subsubsection{Displacements in $E_{3}$}

When looking at relative displacements, one must consider two bodies with coordinate reference frames affixed to them. Craig [14] defines a reference frame as a set of four vectors providing position and orientation information. One vector describes the position relative to some coordinate system and the other three vectors are mutually orthogonal unit vectors along the principle $X, Y$ and $Z$ axes of the frame. The frame is a description of one coordinate system relative to another. This being said, consider reference frame $\Sigma$ affixed to one of the bodies, which is called the base and will remain fixed for the purposes of this discussion. Reference frame $\Sigma^{\prime}$ is affixed to the second body which moves relative to $\Sigma$.

Using cartesian coordinates, a point $\mathbf{p}^{\prime}$, given by a $3 \times 1$ position vector in $\Sigma^{\prime}$ can be transformed to a point $\mathbf{p}$, represented by a $3 \times 1$ position vector in $\Sigma$, by utilizing

$$
\mathbf{p}=\mathbf{A} \mathbf{p}^{\prime}+\mathbf{d}
$$

where $\mathbf{d}$ represents the $3 \times 1$ position vector of the origin of $\Sigma^{\prime}$ with respect to $\Sigma$, and $\mathbf{A}$ is a $3 \times 3$ orthogonal rotation matrix. There are many ways to represent the orientation and 
the method used in this thesis will be discussed in the following subsections.

Utilizing homogenous coordinates it is possible to use one single matrix to produce the same results as Equation 2.3. If $\mathbf{p}$ and $\mathbf{p}^{\prime}$ are now represented by homogenous coordinates, the equation becomes

$$
\mathbf{p}=\mathbf{T} \mathbf{p}^{\prime}
$$

which is now linear, where $\mathbf{T}$ takes the form

$$
\mathbf{T}=\left[\begin{array}{cccc}
1 & 0 & 0 & 0 \\
d_{1} & a_{11} & a_{12} & a_{13} \\
d_{2} & a_{21} & a_{22} & a_{31} \\
d_{3} & a_{31} & a_{32} & a_{33}
\end{array}\right]
$$

$d_{i}, i=1, \ldots, 3$ are the elements of position vector $\mathbf{d}$ and $a_{j k}, j, k=1, \ldots, 3$ are the elements of the rotation matrix $\mathbf{A}$. The group of all displacements in $E_{3}$ is called $S E(3)$ and is represented by this transformation.

One possible parametrization of the rotations is given using Euler angles. This method uses three rotations about the moving coordinate axes to define the rotation. This method is not used in this thesis, but more information is provided by Bottema and Roth [15]. Another representation utilizes quaternions which are used to provide a singularity-free representation of rotations in $E_{3}$. In order to understand this an overview of quaternions and how they are used to represent rotations in $E_{3}$ is required and is provided in Section 2.4.4, but first the concept of groups must be introduced.

\subsubsection{Groups}

Before introducing quaternions a quick introduction to groups is required. A group $G(\mathcal{G}, *)$ is a set $\mathcal{G}$ of elements that are all related by the binary operation $*$ that has the following properties [4].

- if $a, b \in G$, then $a * b \in G$; 
- if $a, b, c \in G$, then $a *(b * c)=(a * b) * c$;

- the identity $i$ of $G$ under $*$, exists such that $a * i=i * a=a$ for $a \in G$; and

- for all $a \in G$, the inverse of $a$ under $*, a^{-1} \in G$, exists such that $a * a^{-1}=a^{-1} * a=i$.

\subsubsection{Quaternions and Representation of Rotations in $E_{3}$}

Introduced by W.R. Hamilton [16], quaternions were originally intended to describe the relative position of two vectors in three dimensional space. They are essentially the three dimensional equivalent to complex numbers in the plane. The use of quaternions to represent rotations is described by Pfurner in [2].

A quaternion is represented by an ordered quadruple of real numbers, such as $P=$ $\left(p_{0}, p_{1}, p_{2}, p_{3}\right), p_{i} \in \mathbb{R}$ and $i \in 0 \ldots, 3$, with $\mathbb{R}$ being the set of all real numbers. The set of all such quadruples is called $\mathbb{H}$. The $p_{0}$ element of $P$ is called the scalar component and the remaining components form the vector component, $\mathbf{p}=\left(p_{1}, p_{2}, p_{3}\right)$. A quaternion is called vectorial if the scalar component is equal to zero.

To illustrate the definitions of quaternion operations, let $P=\left(p_{0}, p_{1}, p_{2}, p_{3}\right)$ and $Q=$ $\left(q_{0}, q_{1}, q_{2}, q_{3}\right)$. Addition and subtraction are component wise, while multiplication is defined by

$$
\begin{aligned}
P * Q= & \left(p_{0} q_{0}-p_{1} q_{1}-p_{2} q_{2}-p_{3} q_{3}, p_{0} q_{1}+p_{1} q_{0}+p_{2} q_{3}-p_{3} q_{2}\right. \\
& \left.p_{0} q_{2}-p_{1} q_{3}+p_{2} q_{0}+p_{3} q_{1}, p_{0} q_{3}+p_{1} q_{2}-p_{2} q_{1}+p_{3} q_{0}\right)
\end{aligned}
$$

An alternative representation of a quaternion is

$$
P=p_{0} \mathbf{1}+p_{1} \mathbf{i}+p_{2} \mathbf{j}+p_{3} \mathbf{k}=p_{0}+\mathbf{p}
$$

where $\mathbf{1}=(1,0,0,0), \mathbf{i}=(0,1,0,0), \mathbf{j}=(0,0,1,0)$ and $\mathbf{k}=(0,0,0,1)$. It is important to note that $\mathbf{i}, \mathbf{j}$ and $\mathbf{k}$ are defined in quaternion algebra where the fundamental special products are $\mathbf{i}^{2}=\mathbf{j}^{2}=\mathbf{k}^{2}=\mathbf{i j k}=-1, \mathbf{i j}=\mathbf{k}=-\mathbf{j i}, \mathbf{j k}=\mathbf{i}=-\mathbf{k} \mathbf{j}$, and $\mathbf{k i}=\mathbf{j}=-\mathbf{i k}$. Defining the conjugate of a quaternion, which is analogous to the conjugate of a complex 
number, as

$$
\widetilde{P}=p_{0} \mathbf{1}-p_{1} \mathbf{i}-p_{2} \mathbf{j}-p_{3} \mathbf{k}
$$

leads to the norm being defined as

$$
\|P\|=\sqrt{P * \widetilde{P}}=\sqrt{p_{0}^{2}+p_{1}^{2}+p_{2}^{2}+p_{3}^{2}}
$$

and the inverse defined as

$$
P^{-1}=\frac{\widetilde{P}}{\|P\|^{2}} .
$$

when $\|P\|=1$, the quaternion is said to be normalized and $P^{-1}=\widetilde{P}$.

With an understanding of the basic quaternion operations it is possible to understand how they are used to describe rotations. Euler used what amounts to normalized quaternions to define the rotation of vector $\mathbf{q}$ in $E_{3}$ about an axis with direction given by $\mathbf{p}$ as

$$
\operatorname{Ad} P(\mathbf{q})=P * \mathbf{q} * \widetilde{P}=P * \mathbf{q} * P^{-1}
$$

where $\operatorname{Ad} P(\mathbf{q})$ is defined as the quaternion rotation operator, $P$ is a normalized quaternion and $\mathbf{q}$ is a vectorial quaternion. $P$ may be represented by $P=\cos \left(\frac{\theta}{2}\right)+\mathbf{p} \sin \left(\frac{\theta}{2}\right)$ and describes a rotation of $\mathbf{q}$ about $\mathbf{p}$ by angle $\theta$.

The orthogonal rotation matrix representing the group of rotations, $S O(3)$, can now be obtained by rotating the unit vectors $\mathbf{i}, \mathbf{j}$ and $\mathbf{k}$ about the axis described by the normalized quaternion $X=x_{0} \mathbf{1}+x_{1} \mathbf{i}+x_{2} \mathbf{j}+x_{3} \mathbf{k}$. First looking at the rotation of each axis the following is obtained:

$$
\begin{aligned}
X * \mathbf{i} * \widetilde{X} & =\left(x_{0} \mathbf{1}+x_{1} \mathbf{i}+x_{2} \mathbf{j}+x_{3} \mathbf{k}\right) \mathbf{i}\left(x_{0} \mathbf{1}-x_{1} \mathbf{i}-x_{2} \mathbf{j}-x_{3} \mathbf{k}\right) \\
& =\left(x_{0} \mathbf{i}-x_{1} \mathbf{1}-x_{2} \mathbf{k}+x_{3} \mathbf{j}\right)\left(x_{0} \mathbf{1}-x_{1} \mathbf{i}-x_{2} \mathbf{j}-x_{3} \mathbf{k}\right) \\
& =\left(x_{0}^{2}+x_{1}^{2}-x_{2}^{2}-x_{3}^{2}\right) \mathbf{i}+2\left(x_{1} x_{2}+x_{0} x_{3}\right) \mathbf{j}+2\left(x_{1} x_{3}-x_{0} x_{2}\right) \mathbf{k} \\
X * \mathbf{j} * \widetilde{X} & =2\left(x_{1} x_{2}-x_{0} x_{3}\right) \mathbf{i}+\left(x_{0}^{2}-x_{1}^{2}+x_{2}^{2}-x_{3}^{2}\right) \mathbf{j}+2\left(x_{2} x_{3}+x_{0} x_{1}\right) \mathbf{k} \\
X * \mathbf{k} * \widetilde{X} & =2\left(x_{1} x_{3}+x_{0} x_{2}\right) \mathbf{i}+2\left(x_{2} x_{3}-x_{0} x_{1}\right) \mathbf{j}+\left(x_{0}^{2}-x_{1}^{2}-x_{2}^{2}+x_{3}^{2}\right) \mathbf{k}
\end{aligned}
$$


The coefficients of the resulting quaternions in Equation (2.7) can now be assembled into a matrix that represents $\mathrm{SO}(3)$. This matrix is

$$
A d X=\left[\begin{array}{ccc}
x_{0}^{2}+x_{1}^{2}-x_{2}^{2}-x_{3}^{2} & 2\left(x_{1} x_{2}-x_{0} x_{3}\right) & 2\left(x_{1} x_{3}+x_{0} x_{2}\right) \\
2\left(x_{1} x_{2}+x_{0} x_{3}\right) & x_{0}^{2}-x_{1}^{2}+x_{2}^{2}-x_{3}^{2} & 2\left(x_{2} x_{3}-x_{0} x_{1}\right) \\
2\left(x_{1} x_{3}-x_{0} x_{2}\right) & 2\left(x_{2} x_{3}+x_{0} x_{1}\right) & x_{0}^{2}-x_{1}^{2}-x_{2}^{2}+x_{3}^{2}
\end{array}\right]
$$

where the parameters $x_{i}, i=0, \ldots, 3$ are known as Euler-Rodrigues parameters.

This is one representation of rotations about the origin. A method for obtaining the Euler-Rodrigues parameters will be shown in Section 2.4.6, but first a parametrization of $\mathrm{SE}(3)$ is required. The concept of dual quaternions can be used to do this.

\subsubsection{Dual Quaternions and Representing Displacements in $E_{3}$}

As is shown by Bottema and Roth [15] and Pfurner [2], $S E(3)$ can be represented in a way that is free from parametrization singularities by using dual quaternions, also known as biquaternions. In this section the necessary characteristics of dual quaternions will be defined and then used to show the parametrization of general displacements in $E_{3}$.

The dual quaternion is created by substituting dual numbers for the coefficients of a quaternion. A dual number is written as $a_{i}=x_{i}+\varepsilon y_{i}$, where $\varepsilon^{2}=0$. At this time it is useful to look at the algebra of dual numbers as described by Bottema and Roth [15]. The dual numbers posses the following characteristics if $a_{1}$ and $a_{2}$ are both dual numbers:

- $0 \varepsilon=\varepsilon 0=0$

- $a \varepsilon=\varepsilon a$

- $\left(x_{1}+\varepsilon y_{1}\right)+\left(x_{2}+\varepsilon y_{2}\right)=x_{1}+x_{2}+\varepsilon\left(y_{1}+y_{2}\right)$

- $\left(x_{1}+\varepsilon y_{1}\right)\left(x_{2}+\varepsilon y_{2}\right)=x_{1} x_{2}+\varepsilon\left(x_{1} y_{2}+x_{2} y_{1}\right) ;$ and

- if $x_{1}+\varepsilon y_{1}=x_{2}+\varepsilon y_{2}$, then $x_{1}=x_{2}$ and $y_{1}=y_{2}$.

Division of dual numbers is not always possible because the product of two dual numbers 
may be zero even if neither dual number is equal to zero. This is because of the fact that $\varepsilon$ was defined such that $\varepsilon^{2}=0$, therefore $\left(\varepsilon y_{1}\right)\left(\varepsilon y_{2}\right)=0$ for any $y_{1}$ and $y_{2}$.

Substituting a dual number into a quaternion as previously defined gives

$$
\begin{aligned}
P & =\left(x_{0}+\varepsilon y_{0}\right) \mathbf{1}+\left(x_{1}+\varepsilon y_{1}\right) \mathbf{i}+\left(x_{2}+\varepsilon y_{2}\right) \mathbf{j}\left(x_{3}+\varepsilon y_{3}\right) \mathbf{k} \\
& =\mathbf{a}+\mathbf{p}
\end{aligned}
$$

where $\mathbf{a}=x_{0}+\varepsilon y_{0}$ and $\mathbf{p}=\mathbf{p}_{1}+\varepsilon \mathbf{p}_{2}$ with $\mathbf{p}_{1}=\left(x_{1}, x_{2}, x_{3}\right)$ and $\mathbf{p}_{2}=\left(y_{1}, y_{2}, y_{3}\right)$. All elements of a dual quaternion are dual numbers.

The product of dual quaternions $P=\mathbf{a}+\mathbf{p}$ and $Q=\mathbf{b}+\mathbf{q}$ is

$$
P * Q=\mathbf{a b}-\mathbf{p q}+\mathbf{a p}+\mathbf{b q}+\mathbf{p} \times \mathbf{q}
$$

where $\mathbf{p} \times \mathbf{q}$ is the cross product of vectors $\mathbf{p}$ and $\mathbf{q}$. Dual quaternions can be conjugated in two different ways,

$$
\widetilde{P}=x_{0}+\varepsilon y_{0}-\mathbf{p}_{1}-\varepsilon \mathbf{p}_{2}
$$

and

$$
P_{\varepsilon}=x_{0}-\varepsilon y_{0}+\mathbf{p}_{\mathbf{1}}-\varepsilon \mathbf{p}_{\mathbf{2}}
$$

The norm of the dual quaternion is defined as

$$
\|P\|=\sqrt{P * \widetilde{P}}
$$

where the product under the root is

$$
\begin{aligned}
P * \widetilde{P} & =\mathbf{a}^{2}+\mathbf{p} \mathbf{p} \\
& =x_{0}^{2}+2 \varepsilon x_{0} y_{0}+\mathbf{p}_{\mathbf{1}} \mathbf{p}_{1}+2 \varepsilon \mathbf{p}_{1} \mathbf{p}_{\mathbf{2}} \\
& =x_{0}^{2}+\mathbf{p}_{\mathbf{1}} \mathbf{p}_{\mathbf{1}}+2 \varepsilon\left(x_{0} y_{0}+\mathbf{p}_{\mathbf{1}} \mathbf{p}_{\mathbf{2}}\right) \\
& =x_{0}^{2}+x_{1}^{2}+x_{2}^{2}+x_{3}^{2}+\varepsilon\left(x_{0} y_{0}+x_{1} y_{1}+x_{2} y_{2}+x_{3} y_{3}\right)
\end{aligned}
$$

As with quaternions, the condition for a dual quaternion to be called normalized is $\|P\|=1$, 
which means $x_{0}^{2}+x_{1}^{2}+x_{2}^{2}+x_{3}^{2}=1$ and $x_{0} y_{0}+x_{1} y_{1}+x_{2} y_{2}+x_{3} y_{3}=0$.

When it is defined that $X=\left(x_{0}, x_{1}, x_{2}, x_{3}\right), Y=\left(y_{0}, y_{1}, y_{2}, y_{3}\right) \in \mathbb{H}$ then it can be said that $P=X+\varepsilon Y$. If $\mathbb{V}$ is defined as the set of all dual quaternions with the form $1+\varepsilon \mathbf{r}$ with $\mathbf{r}$ a vector of $\mathbb{R}^{3}$, then the mapping from $\mathbb{V}$ to $\mathbb{H}$ is

$$
\begin{aligned}
A d_{e} P(\mathbf{r}) & =P_{\varepsilon} \mathbf{r} \widetilde{P} \\
& =(X-\varepsilon Y)(1+\varepsilon \mathbf{r})(\widetilde{X}+\varepsilon \widetilde{Y}) \\
& =1+\varepsilon(X \mathbf{r} \widetilde{X}+X \tilde{Y}-Y \widetilde{X}) .
\end{aligned}
$$

Pfurner [2] shows that $A d_{e}$ is a mapping of the set of normalized dual quaternions, $\mathbb{U}_{d}$, into the group $S E(3) . \mathbb{U}_{d}$ is a group with the inverse element being the conjugate quaternion. The $X \mathbf{r} \tilde{X}$ term is the quaternion description of a rotation about the origin and the $X \tilde{Y}-Y \tilde{X}$ term represents the translational part of a displacement in $E_{3}$. Expanding the translational component yields

$$
\begin{gathered}
X \tilde{Y}-Y \tilde{X}=2\left(-x_{0} y_{1}+x_{1} y_{0}-x_{2} y_{3}+x_{3} y_{2}\right) \mathbf{i}+2\left(-x_{0} y_{2}+x_{1} y_{3}+x_{2} y_{0}-x_{3} y_{1}\right) \mathbf{j} \\
+2\left(-x_{0} y_{3}-x_{1} y_{2}+x_{2} y_{1}+x_{3} y_{0}\right) \mathbf{k}
\end{gathered}
$$

Utilizing homogenous coordinates and Equation (2.8), the matrix representation of displacements in $S E(3)$ is

$$
\mathbf{A}=\left[\begin{array}{cccc}
1 & 0 & 0 & 0 \\
l & x_{0}^{2}+x_{1}^{2}-x_{2}^{2}-x_{3}^{2} & 2\left(x_{1} x_{2}-x_{0} x_{3}\right) & 2\left(x_{1} x_{3}+x_{0} x_{2}\right) \\
m & 2\left(x_{1} x_{2}+x_{0} x_{3}\right) & x_{0}^{2}-x_{1}^{2}+x_{2}^{2}-x_{3}^{2} & 2\left(x_{2} x_{3}-x_{0} x_{1}\right) \\
n & 2\left(x_{1} x_{3}-x_{0} x_{2}\right) & 2\left(x_{2} x_{3}+x_{0} x_{1}\right) & x_{0}^{2}-x_{1}^{2}-x_{2}^{2}+x_{3}^{2}
\end{array}\right]
$$

where

$$
\begin{aligned}
l & =2\left(-x_{0} y_{1}+x_{1} y_{0}-x_{2} y_{3}+x_{3} y_{2}\right) \\
m & =2\left(-x_{0} y_{2}+x_{1} y_{3}+x_{2} y_{0}-x_{3} y_{1}\right) \\
n & =2\left(-x_{0} y_{3}-x_{1} y_{2}+x_{2} y_{1}+x_{3} y_{0}\right)
\end{aligned}
$$

To obtain the matrix representation of the displacements of $S E(3)$, the normalizing 
condition may be removed and left as $x_{0}^{2}+x_{1}^{2}+x_{2}^{2}+x_{3}^{2} \neq 0$ and $\mathbf{A}$ becomes

$$
\mathbf{A}=\frac{1}{\Delta}\left[\begin{array}{cccc}
x_{0}^{2}+x_{1}^{2}+x_{2}^{2}+x_{3}^{2} & 0 & 0 & 0 \\
l & x_{0}^{2}+x_{1}^{2}-x_{2}^{2}-x_{3}^{2} & 2\left(x_{1} x_{2}-x_{0} x_{3}\right) & 2\left(x_{1} x_{3}+x_{0} x_{2}\right) \\
m & 2\left(x_{1} x_{2}+x_{0} x_{3}\right) & x_{0}^{2}-x_{1}^{2}+x_{2}^{2}-x_{3}^{2} & 2\left(x_{2} x_{3}-x_{0} x_{1}\right) \\
n & 2\left(x_{1} x_{3}-x_{0} x_{2}\right) & 2\left(x_{2} x_{3}+x_{0} x_{1}\right) & x_{0}^{2}-x_{1}^{2}-x_{2}^{2}+x_{3}^{2}
\end{array}\right]
$$

where $\Delta=x_{0}^{2}+x_{1}^{2}+x_{2}^{2}+x_{3}^{2}$. The parameters $x_{i}, y_{i}, i=0, \ldots, 3$ are called the Study Parameters or soma coordinates. Using the algebra of dual quaternions means that the mapping $A d_{e}$ is 2 to 1 . This leads to the fact that there are two dual quaternions, $P$ and $-P$, describing every displacement in $E_{3}$. As noted by Brunnthaler [4] the terms $X \tilde{Y}-Y \tilde{X}$ and $X \mathbf{r} \widetilde{X}$ are vector quaternions which are equal to their negative quaternions. The concept of kinematic mapping is now introduced and the issue of 2 to 1 mapping will be addressed within this new concept.

\subsubsection{Kinematic Mapping}

The representation of $E_{3}$ displacements in $P^{7}$ utilizes the Study parameters $\left(x_{0}, x_{1}, x_{2}, x_{3}, y_{0}, y_{1}, y_{2}, y_{3}\right)$ which must satisfy the condition

$$
x_{0} y_{0}+x_{1} y_{1}+x_{2} y_{2}+x_{3} y_{3}=0 \text {. }
$$

This includes the trivial case $x_{0}=x_{1}=x_{2}=x_{3}=0$, called the exceptional generator, which does not represent a displacement in $E_{3}$ because all of the Euler parameters representing the rotation about the origin would be zero, a situation having no physical meaning. Equation (2.12) with the exceptional generator removed is called the Study quadric, represented by $S_{6}^{2}$ and can be viewed as a hyperboloid in $P^{7}$. Any displacement in $E_{3}$ maps to a single unique point on $S_{6}^{2}$. The mapping of displacements from $S E(3)$ to $S_{6}^{2}$ is called kinematic mapping [15].

In the previous section it was stated the mapping is 2 to 1 , this becomes obvious here because normalized dual quaternions $P$ and $-P$ describe the same point on $S_{6}^{2}$. This is 
resolved by looking at the eight terms of the dual quaternions as the homogenous coordinates of a point in seven-dimensional projective space $P^{7}$, called the kinematic image space. The displacements in $E_{3}$ are now represented one to one in $P^{7}$.

Kinematic mapping is used in this thesis to represent the displacements obtainable by a mechanism as a set of related points in the kinematic image space. This is the concept of constraint varieties which will be presented in detail in Section 2.7, but for this section it is important to understand that there is a need to map from displacements in $E_{3}$ represented by the matrix $\mathbf{T}$ in Equation (2.4) to the points lying on $S_{6}^{2}$. A method for obtaining the Study parameters for a displacement represented by $\mathbf{T}$ is therefore required.

\subsubsection{Derivation of Study Parameters}

Two methods of obtaining the Study parameters are presented here. The first is the most commonly used method employing skew symmetric matrices and the second is the method developed by Study [17]. Study's method is used throughout the remainder of this thesis as its use is very straightforward and overcomes a major shortcoming of the common method. The skew symmetric matrix method is shown for completeness and its use produces equivalent results to Study's method.

The theorem of Cayley states that any orthogonal matrix A may be decomposed such that

$$
\mathbf{A}=(\mathbf{I}-\mathbf{S})^{-1}(\mathbf{I}+\mathbf{S})
$$

where $\mathbf{I}$ is the $3 \times 3$ identity matrix and $\mathbf{S}$ is a skew symmetric matrix of the form

$$
\mathbf{S}=\left[\begin{array}{ccc}
0 & -b_{3} & b_{2} \\
b_{3} & 0 & -b_{1} \\
-b_{2} & b_{1} & 0
\end{array}\right]
$$

For an orthogonal matrix $\mathbf{A}$ with eigenvalues not equal to $-1, \mathbf{S}$ can be computed with $\mathbf{S}=(\mathbf{A}-\mathbf{I})(\mathbf{A}+\mathbf{I})^{-1}$. The entries of $\mathbf{S}$ describe the rotation in $E_{3}$ of angle $\phi$ about the axis represented by the vector $\left(b_{1}, b_{2}, b_{3}\right)$, where $\tan \left(\frac{\phi}{2}\right)=\sqrt{b_{1}^{2}+b_{2}^{2}+b_{3}^{2}}$. When the $b_{i}, i=1,2,3$ 
terms are made homogenous such that $b_{i}=\frac{x_{i}}{x_{0}}$ they become the Euler-Rodrigues parameters. From Craig [14] it is shown that the Euler-Rodrigues parameters are given by:

$$
\begin{aligned}
& x_{0}=\cos \frac{\phi}{2}, \\
& x_{1}=b_{1} \sin \frac{\phi}{2}, \\
& x_{2}=b_{2} \sin \frac{\phi}{2}, \\
& x_{3}=b_{3} \sin \frac{\phi}{2} .
\end{aligned}
$$

The property $x_{0}^{2}+x_{1}^{2}+x_{2}^{2}+x_{3}^{2} \neq 0$ must always hold.

In the event that the rotation angle $\phi$ approaches any multiple of $\pi$ this method no longer holds because an eigenvalue of $\mathbf{A}$ is -1 and $\tan \left(\frac{\phi}{2}\right)$ goes to infinity. In such situations, an alternative method is required to obtain the Euler-Rodrigues parameters, such as using limits when $\phi$ approaches a singularity.

The Study method for obtaining the Euler-Rodrigues parameters is very straightforward. The ratios

$$
\begin{aligned}
x_{0}: x_{1}: x_{2}: x_{3} & =1+a_{11}+a_{22}+a_{33}: a_{32}-a_{23}: a_{13}-a_{31}: a_{21}-a_{12} \\
& =a_{32}-a_{23}: 1+a_{11}-a_{22}-a_{33}: a_{12}+a_{21}: a_{31}+a_{13} \\
& =a_{13}-a_{31}: a_{12}+a_{21}: 1-a_{11}+a_{22}-a_{33}: a_{23}+a_{32} \\
& =a_{21}-a_{12}: a_{31}+a_{13}: a_{23}+a_{32}: 1-a_{11}-a_{22}+a_{33} .
\end{aligned}
$$

are found from the $3 \times 3$ lower-right sub-matrix of $\mathbf{T}$ in Equation (2.4). The derivation of these ratios is shown by Pfurner in [2]. In general the four ratios of Equation (2.14) will yield the same results, but in special cases, such as when $\phi$ is a multiple of $\pi$ one or more, but not all of the ratios may be $0: 0: 0: 0$. Thus the Euler-Rodrigues parameters can always be found directly from $\mathbf{T}$.

The remaining Study parameters, $y_{0}, y_{1}, y_{2}, y_{3}$, can now be found from the set of equations that includes the $l, m, n$ terms of $\mathbf{A}$ in Equation (2.11) set equal to the corresponding terms of $\mathbf{T}$ in Equation (2.4) and the Study quadric of Equation (2.12). Solving this set for 
the remaining Study parameters gives

$$
\begin{aligned}
& y_{0}=\frac{1}{2}\left(d_{1} x_{1}+d_{2} x_{2}+d_{3} x_{3}\right) \\
& y_{1}=\frac{1}{2}\left(-d_{1} x_{0}+d_{3} x_{2}-d_{2} x_{3}\right) \\
& y_{2}=\frac{1}{2}\left(-d_{2} x_{0}-d_{3} x_{1}+d_{1} x_{3}\right) \\
& y_{3}=\frac{1}{2}\left(-d_{3} x_{0}+d_{2} x_{1}-d_{1} x_{2}\right),
\end{aligned}
$$

where $d_{1}, d_{2}$ and $d_{3}$ are defined in Equation (2.4).

\subsection{Denavit Hartenberg Parameters and Direct Kinematics}

When working with serial manipulators it is necessary to have a unambiguous mathematical description of the kinematic chain. This can be obtained using Denavit-Hartenberg parameters or more simply DH-parameters. The procedure presented here is for labeling an $n$ R-chain, the interested reader is referred to Denavit and Hartenberg [18], Craig [14] or Shigley and Uicker [3] for a more detailed description.

\subsubsection{Assigning Reference Frames and DH-Parameters}

The labeling procedure begins by numbering each link in the kinematic chain with the base being link 0 , link 1 being the next link, and so on up to link $n$. Next, each joint axis in the chain and the common normal between each axis are established. The axes are numbered sequentially from 1 to $n$, starting with the joint between the base and link 1 .

Each link is assigned one reference frame, and the assignment of this reference frame depends on the two axes at the ends of the link. For the purposes of this description, each link $i, i=1, \ldots, n$ describes a rigid link that lies on the common normal between axes $i$ and $i+1$, irrespective of the actual shape of the physical link in the manipulator, which is irrelevant to the kinematic analysis presented in this thesis. The special cases of intersecting or parallel axes will be addressed as needed.

Let frame $i$ be denoted $\Sigma_{i}$. The origin of $\Sigma_{i}$ established where link $i$ intersects the joint axes $i$. The $Z_{i}$-axis of $\Sigma_{i}$ points along the $i$ axis, the direction is arbitrary, but with 
experience is selected to ease future calculations. The $X_{i}$-axis points along the common perpendicular towards the origin of $\Sigma_{i+1}$. If the axes $i$ and $i+1$ intersect, the axis $X_{i}$ is parallel to the normal of to the intersecting axes, the direction is again selected to ease

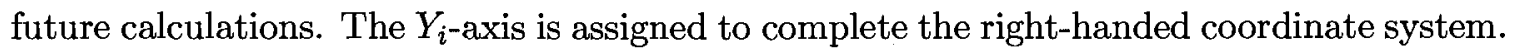
This procedure works well for intermediate links, however the base and EE frames, $\Sigma_{0}$ and $\Sigma_{n}$ respectively, are often selected to ease calculations by setting as many DH-parameters to zero as possible.

Once the link frames have been established the four DH-parameters are defined as follows:

Link length $a_{i}$, the directed distance from axis $i-1$ to $i$ along $X_{i}$;

Link twist $\alpha_{i}$, the directed angle from axis $i-1$ to $i$ about $X_{i}$;

Joint offset $d_{i}$, the directed distance from the origin of $\Sigma_{i-1}$ to the intersection of axis $i-1$ and $X_{i}$ along $Z_{i-1}$;

Joint angle $\theta_{i}$, the directed angle from $X_{i-1}$ to $X_{i}$ about $Z_{i-1}$.

Figure 2.5 shows a typical link with reference frames and the DH-parameters identified.

In an $n \mathrm{R}$-chain, the values $\theta_{i}, i=1 \ldots n$ are variable as the links rotate about the joint, and called the joint variables, while the remaining three parameters are fixed and are called the link and joint parameters. For a P-pair, the $d_{i}$ parameter becomes the joint variable.

With the four DH-parameters assigned for each link, the kinematic chain is unambiguously defined and the direct kinematic equations can be obtained.

\subsubsection{Direct Kinematics of $n$ R-Chains}

For each link in an $n \mathrm{R}$ chain, a matrix is established using the link parameters that represent the geometry of the link. The following method for assembling the DH-parameters into matrices and obtaining the direct kinematics equations is well known, see Husty, et al. [19], for example. For link $i, i=1, \ldots, n$ the matrix of link parameters, $\mathbf{G}_{\mathbf{i}}$, is assembled as 


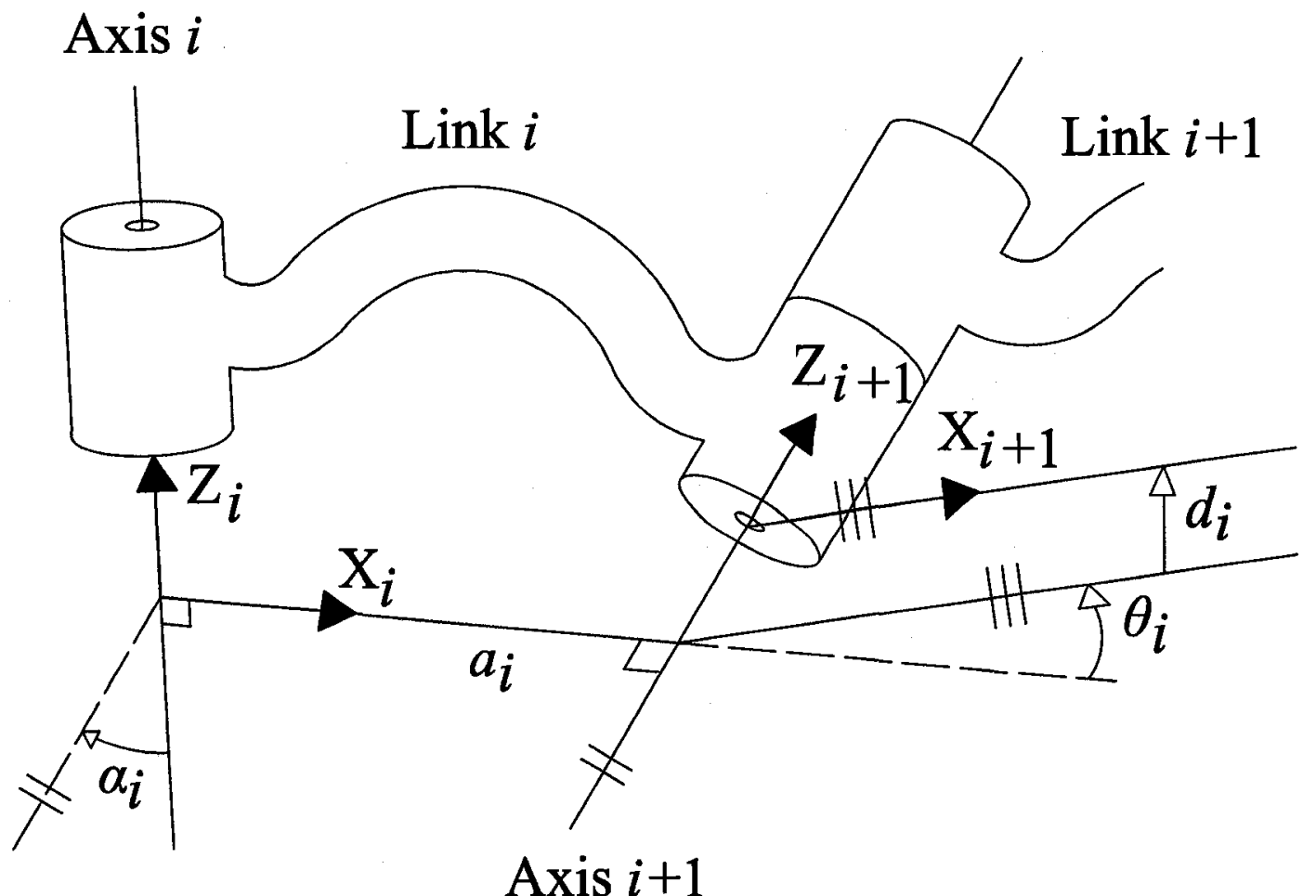

Figure 2.5: Link with reference frames and DH-parameters.

follows:

$$
\mathbf{G}_{\mathbf{i}}=\left[\begin{array}{cccc}
1 & 0 & 0 & 0 \\
a_{i} & 1 & 0 & 0 \\
0 & 0 & \cos \left(\alpha_{i}\right) & -\sin \left(\alpha_{i}\right) \\
d_{i} & 0 & \sin \left(\alpha_{i}\right) & \cos \left(\alpha_{i}\right)
\end{array}\right]
$$

The joint variables are assembled into matrices, $\mathbf{M}_{\mathbf{i}}$, for each joint as follows:

$$
\mathbf{M}_{\mathbf{i}}=\left[\begin{array}{cccc}
1 & 0 & 0 & 0 \\
0 & \cos \left(\theta_{i}\right) & -\sin \left(\theta_{i}\right) & 0 \\
0 & \sin \left(\theta_{i}\right) & \cos \left(\theta_{i}\right) & 0 \\
0 & 0 & 0 & 1
\end{array}\right]
$$

With these matrices defined it is now possible to obtain the direct kinematic equations. 
In matrix form the equations are

$$
{ }^{0} \mathbf{T}_{\mathbf{n}}=\mathbf{B M}_{1} \mathbf{G}_{1} \ldots \mathbf{M}_{\mathbf{i}} \mathbf{G}_{\mathbf{i}} \ldots \mathbf{M}_{\mathbf{n}} \mathbf{G}_{\mathbf{n}}
$$

where ${ }^{0} \mathbf{T}_{\mathbf{n}}$ is the transformation matrix which describes the pose of $\Sigma_{n}$, the EE reference frame, relative to the base reference frame $\Sigma_{0}$ and $\mathbf{B}$ is the transformation from $\Sigma_{1}$ to $\Sigma_{0}$.

${ }^{0} \mathbf{T}_{\mathbf{n}}$ is the matrix representation of the direct kinematic equations in the homogenous coordinates of $E_{3}$.

\subsection{Effect of Transformations in $E_{3}$ on Points in $P^{7}$}

In this thesis it is necessary to understand the effect of a coordinate transformation in $E_{3}$ on the Study parameters in $P^{7}$. That is, if the Study parameters of a displacement are known for a specific coordinate system and that system changes, what is the effect on the Study parameters? The two important transformations are transformations in the base reference frame, which results in a change of the fixed coordinate system, and in the moving reference frame, which results in a change of the $\mathrm{EE}$ coordinate system. Each type of transformation has a different influence on the the Study parameters in $P^{7}$ and must be examined separately.

In both cases the following two matrices are used. The first, $\mathbf{A}$, describes $\Sigma_{E E}$ relative to $\Sigma_{0}$ and is built by substituting the Study parameters $\mathbf{a}=\left(a_{0}, a_{1}, a_{2}, a_{3}, a_{4}, a_{5}, a_{6}, a_{7}\right)^{T}$ for $\left(x_{0}, x_{1}, x_{2}, x_{3}, y_{0}, y_{1}, y_{2}, y_{3}\right)^{T}$ in Equation (2.11). Similarly, the coordinate transformation is described by matrix $\mathbf{T}$ which is built by substituting $\mathbf{t}=\left(t_{0}, t_{1}, t_{2}, t_{3}, t_{4}, t_{5}, t_{6}, t_{7}\right)^{T}$ for $\left(x_{0}, x_{1}, x_{2}, x_{3}, y_{0}, y_{1}, y_{2}, y_{3}\right)^{T}$. A can be thought of as the result of Equation (2.18), with a being the Study parameters of $\mathbf{A}$, while $\mathbf{T}$ represents some form of change in the kinematic architecture of a kincmatic chain, such as moving the entire manipulator relative to the fixed coordinate system or changing the architecture of the links and altering the DH-parameters. Moving the whole manipulator to a new position represents a transformation in the base frame, while altering the DH-parameters is a transformation in the moving frame. The 
elements of $\mathbf{t}$ are the Study parameters of $\mathbf{T}$.

\subsubsection{Transformation in the Base Frame}

A transformation in the base frame is described by the matrix product TA. After the matrix multiplication the Study parameters are found using Study's method as presented in Section 2.4.6. The resulting Study parameters are

$$
\mathbf{t}_{b}=\Delta\left(\begin{array}{c}
a_{0} t_{0}-a_{1} t_{1}-a_{2} t_{2}-a_{3} t_{3} \\
a_{0} t_{1}+a_{1} t_{0}+a_{3} t_{2}-a_{2} t_{3} \\
a_{0} t_{2}+a_{2} t_{0}+a_{1} t_{3}-a_{3} t_{1} \\
a_{0} t_{3}+a_{3} t_{0}+a_{2} t_{1}-a_{1} t_{2} \\
a_{0} t_{4}-a_{1} t_{5}-a_{2} t_{6}-a_{3} t_{7}+a_{4} t_{0}-a_{5} t_{1}-a_{6} t_{2}-a_{7} t_{3} \\
a_{0} t_{5}+a_{1} t_{4}-a_{2} t_{7}+a_{3} t_{6}+a_{4} t_{1}+a_{5} t_{0}-a_{6} t_{3}+a_{7} t_{2} \\
a_{0} t_{6}+a_{1} t_{7}+a_{2} t_{4}-a_{3} t_{5}+a_{4} t_{2}+a_{5} t_{3}+a_{6} t_{0}-a_{7} t_{1} \\
a_{0} t_{7}-a_{1} t_{6}+a_{2} t_{5}+a_{3} t_{4}+a_{4} t_{3}-a_{5} t_{2}+a_{6} t_{1}+a_{7} t_{0}
\end{array}\right)
$$

where

$$
\Delta=\frac{a_{0} t_{0}-a_{1} t_{1}-a_{2} t_{2}-a_{3} t_{3}}{\left(a_{0}^{2}+a_{1}^{2}+a_{2}^{2}+a_{3}^{2}\right)\left(t_{0}^{2}+t_{1}^{2}+t_{2}^{2}+t_{3}^{2}\right)}
$$

The $\Delta$ term may be ignored because homogeneous coordinates are being used. The subscript $b$ indicates that the transformation is in the base frame. 
Equation (2.19) can be written as $\mathbf{T}_{b} \mathbf{a}$, where

$$
\mathbf{T}_{b}=\left[\begin{array}{cccccccc}
t_{0} & -t_{1} & -t_{2} & -t_{3} & 0 & 0 & 0 & 0 \\
t_{1} & t_{0} & -t_{3} & -t_{2} & 0 & 0 & 0 & 0 \\
t_{2} & t_{3} & t_{0} & -t_{1} & 0 & 0 & 0 & 0 \\
t_{3} & -t_{2} & t_{1} & t_{0} & 0 & 0 & 0 & 0 \\
t_{4} & -t_{5} & -t_{6} & -t_{7} & t_{0} & -t_{1} & -t_{2} & -t_{3} \\
t_{5} & t_{4} & -t_{7} & t_{6} & t_{1} & t_{0} & -t_{3} & t_{2} \\
t_{6} & t_{7} & t_{4} & -t_{5} & t_{2} & t_{3} & t_{0} & -t_{1} \\
t_{7} & -t_{6} & t_{5} & t_{4} & t_{3} & -t_{2} & t_{1} & t_{0}
\end{array}\right] .
$$

With this representation, the effect of a transformation in the base frame on the Study parameters in $P^{7}$ can be found using the Study parameters of the transformation matrix.

\subsubsection{Transformations in the Moving Frame}

The same procedure as for the transformation in the base frame is used in the case of a transformation in the moving frame, however the transformation is now described by the matrix product AT. A similar matrix to $\mathbf{T}_{b}$ is developed called $\mathbf{T}_{m}$, where the subscript $m$ indicates the moving frame, such that the transformed Study parameters are given by $\mathbf{T}_{m} \mathbf{a}$, where

$$
\mathbf{T}_{m}=\left[\begin{array}{cccccccc}
t_{0} & -t_{1} & -t_{2} & -t_{3} & 0 & 0 & 0 & 0 \\
t_{1} & t_{0} & t_{3} & -t_{2} & 0 & 0 & 0 & 0 \\
t_{2} & -t_{3} & t_{0} & t_{1} & 0 & 0 & 0 & 0 \\
t_{3} & t_{2} & -t_{1} & t_{0} & 0 & 0 & 0 & 0 \\
t_{4} & -t_{5} & -t_{6} & -t_{7} & t_{0} & -t_{1} & -t_{2} & -t_{3} \\
t_{5} & t_{4} & t_{7} & -t_{6} & t_{1} & t_{0} & t_{3} & -t_{2} \\
t_{6} & -t_{7} & t_{4} & t_{5} & t_{2} & -t_{3} & t_{0} & t_{1} \\
t_{7} & t_{6} & -t_{5} & t_{4} & t_{3} & t_{2} & -t_{1} & t_{0}
\end{array}\right]
$$

In [2], Pfurner shows and proves many useful properties of the $\mathbf{T}_{b}$ and $\mathbf{T}_{m}$ matrices. 
The most important results include the fact that under a transformation in $E_{3}$ the quadric $S_{6}^{2}$ and the exceptional generator remain unchanged and the inverse of $\mathbf{T}_{b}$ or $\mathbf{T}_{m}$ can be found by substituting $\left(t_{0},-t_{1},-t_{2},-t_{3}, t_{4},-t_{5},-t_{6},-t_{7}\right)$ for $\left(t_{0}, t_{1}, t_{2}, t_{3}, t_{4}, t_{5}, t_{6}, t_{7}\right)$ which results in no change for $t_{0}$ and $t_{4}$.

To describe the matrix $\mathbf{T}_{b}$ or $\mathbf{T}_{m}$ for a displacement described by a $4 \times 4$ matrix, for example $\mathbf{M}_{i}$, the form $\mathbf{T}_{b}\left(\mathbf{M}_{i}\right)$ is used, meaning the $\mathbf{T}_{b}$ matrix is populated by the Study parameters associated with the matrix $\mathbf{M}_{i}$.

\subsection{Constraint Varieties}

Constraint varieties are used in this thesis to describe the displacements, represented by points in $P^{7}$, that a mechanism bound by mechanical constraints can achieve. The EE of a mechanism is constrained to move within its reachable workspace and in the kinematic image space the displacement from the base frame to any unique EE pose is represented by a point. If, as shown in Section 2.4.6, every point on $S_{6}^{2}$ excluding the exceptional generator maps to a displacement, then the set of displacements of a constrained mechanism is a subset of all points on $S_{6}^{2}$. Then, as noted by Hayes, Zsombor-Murray and Chen [13], because a continuous motion is a continuous set of displacements, a constrained motion will map to a continuous set of points on $S_{6}^{2}$ in $P^{7}$. Depending on the nature of the mechanical constraints the set of points in $P^{7}$ obtainable by a mechanism may be represented by a line, surface or higher dimensional algebraic variety.

A definition of varieties is useful at this time, but first the nomenclature for polynomials must be defined. The set of all polynomials in $n$ variables is denoted by $k\left[x_{1}, \ldots, x_{n}\right]$, such that $k$ is any field (for example real numbers, $\mathbb{R}$, natural numbers, $\mathbb{N}$, or complex numbers, $\mathbb{C}$ ) and a polynomial is a finite sum of terms having the form $a x_{1}^{\beta_{1}} \cdots x_{n}^{\beta_{n}}$ with $\beta_{i} \in \mathbb{N}, i=1, \ldots, n$ and $a \in k$. For a finite set of polynomials $\left\{f_{1}, f_{2}, \ldots, f_{s}\right\} \in k\left[x_{1}, \ldots, x_{n}\right]$, the set of all solutions to the system $f_{1}=0, f_{2}=0, \ldots, f_{s}=0$ is called the variety defined by $\left\{f_{1}, f_{2}, \ldots, f_{s}\right\}$, and is represented by $V\left(f_{1}, f_{2}, \ldots, f_{s}\right)$. This leads to the term constraint variety. In this thesis constraint varieties will be given as the intersection of a 
set of polynomials and the intersections occur at the point or set of points in $P^{7}$ where all polynomials in the set are identical to zero. That is, the variety defined by the set of polynomials that result from the mechanical constraints.

The number of polynomials in the set depends on the number of constraints. In $P^{7}$ any point is represented by seven homogeneous parameters as shown in Sections 2.4.4 and 2.4.6 meaning that seven parameters must be defined to define a displacement. If a single constraint is provided, defined by a joint variable, a variable joint angle for an example, with the remaining joint parameters defined constants, then the constraint variety is defined by six polynomials ( $S_{6}^{2}$ and five others.) In some cases, such is in Hayes, Zsombor-Murray and Chen [13], Brunnthaler [4] or Pfurner [2] the constraint varieties may be defined by the intersection of some geometric entity with $S_{6}^{2}$ but for the purposes of this thesis the constraint variety is given as the intersection of a set of polynomials.

A constraint variety may be visualized as a surface in the kinematic mapping image space. The points of the surface represent all possible displacements of the end effector reference frame given the constraints imposed by the kinematic chain. For a planar $2 \mathrm{R}$ chain the constraint variety is a hyperboloid in a three dimensional subspace of the kinematic mapping image space, as illustrated in Figure 2.6.

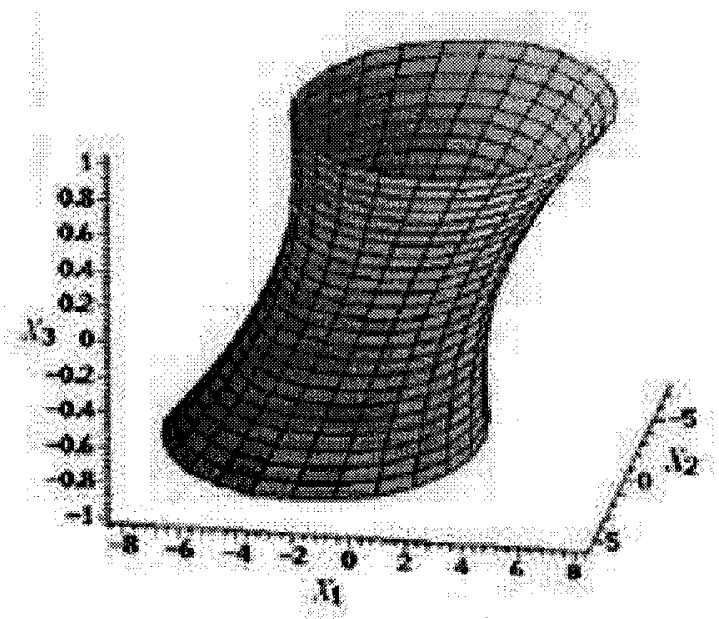

Figure 2.6: Projection of the constraint of an arbitrary planar 2R-chain to a three dimensional subspace of the kinematic mapping image space. 


\subsection{Tangent of the Half-angle Substitution}

Analysis of mechanical systems that possess design or motion parameters described by angles involves equations containing trigonometric functions. Computer algebra systems are generally more efficient with algebraic equations than ones containing trigonometric functions, therefore a method of converting the trigonometric functions to algebraic terms is desired. The process of tangent of the half-angle substitution is commonly used to accomplish this.

Tangent of the half-angle substitution is based on the trigonometric identities

$$
\sin (\phi)=\frac{2 \tan \left(\frac{\phi}{2}\right)}{1+\tan ^{2}\left(\frac{\phi}{2}\right)}
$$

and

$$
\cos (\phi)=\frac{1-\tan ^{2}\left(\frac{\phi}{2}\right)}{1+\tan ^{2}\left(\frac{\phi}{2}\right)},
$$

where $\phi \neq(2 k+1) \pi$ and $k \in\{0,1, \ldots\}$. Substitution of a new variable

$$
u=\tan \left(\frac{\phi}{2}\right)
$$

into the identities provides the following identities

$$
\sin (\phi)=\frac{2 u}{1+u^{2}}
$$

and

$$
\cos (\phi)=\frac{1-u^{2}}{1+u^{2}}
$$

As noted by Pfurner [2] these identities define a mapping of the points of a unit circle parameterized by $\phi$ to the set of real numbers. The inverse mapping is given by

$$
\phi=2 \arctan (u)
$$

From these mappings it can be seen that when $u=0$ then $\phi=0$, when $u=1$ then $\phi=\frac{\pi}{2}$ 
and as $u$ goes to infinity $\phi$ approaches $\pi$.

The tangent of the half-angle substitution technique is utilized throughout this thesis to convert trigonometric functions to rational algebraic functions, a form more suitable for analysis with algebraic software.

\subsection{Resultant Elimination}

An important aspect of this thesis is the intersection of constraint varieties. These intersections are found by solving the system of polynomials describing the intersecting constraint varieties. A commonly employed algebraic method of doing this is by using resultants to eliminate variables from the set of equations in order to obtain a univariate polynomial. This polynomial of one variable can then be solved and back substitution used to obtain the remaining unknown variables for each solution, thus solving the set of equations and yielding intersection of the constraint varieties. The process of using resultants to solve a system of polynomials is provided by Cox, Little and O'Shea [20] and a useful summary of aspects important to constraint variety is given by Pfurner [2].

For the two polynomials

$$
\begin{array}{ll}
f(x)=a_{0} x^{l}+\ldots+a_{l-1} x^{1}+a_{l}, & a_{0} \neq 0, \quad l>0, \\
g(x)=b_{0} x^{m}+\ldots+b_{m-1} x^{1}+b_{m}, & b_{0} \neq 0, \quad m>0,
\end{array}
$$

where the coefficients $a_{i}, i \in\{0, \ldots, l\}$ and $b_{i}, i \in\{0, \ldots, m\}$ may be constants or polynomials in terms of variables other than $x$, the resultant of $f$ and $g$ with respect to $x$ is defined 
to be

$$
\operatorname{Res}_{x}(f, g)=\operatorname{det}\left[\begin{array}{ccccccccc}
a_{0} & & & & b_{0} & & & \\
a_{1} & a_{0} & & & b_{1} & b_{0} & & \\
a_{2} & a_{1} & \ddots & & b_{2} & b_{1} & \ddots & \\
\vdots & a_{2} & \ddots & a_{0} & \vdots & b_{2} & \ddots & b_{0} \\
a_{l} & \vdots & \ddots & a_{1} & b_{m} & \vdots & \ddots & b_{1} \\
& a_{l} & & a_{2} & & b_{m} & & b_{2} \\
& & \ddots & \vdots & & & \ddots & \vdots \\
& & & a_{l} & & & & b_{m}
\end{array}\right],
$$

where the empty elements of the matrix are filled with zeros. The dimensions of the matrix in Equation 2.27 are $(l+m) \times(l+m)$ and the correct number of columns are used to fill these dimensions. If $f$ and $g$ have a common factor then $\operatorname{Res}_{x}(f, g)=0$.

Pfurner uses some examples to illustrate how resultants are used to eliminate variables from systems of equations to solve the systems. The first example illustrates resultant elimination for a system in two equations and two unknowns while the second example is a system of three equations with three variables.

Consider the problem of obtaining the intersection(s) of the two equations

$$
\begin{aligned}
& f(x, y)=x^{2} y-x^{2}-2 x y+x+y-1 \\
& f(x, y)=3 x y-x+5 .
\end{aligned}
$$

The first step is to select a variable to eliminate, in this case $x$, and factor the equations to the form of Equation (2.26) such that they become

$$
\begin{aligned}
& f(x, y)=(y-1) x^{2}+(-2 y+1) x+(y-1) \\
& g(x, y)=(3 y-1) x+5
\end{aligned}
$$


where the coefficients of the powers of $x$ are functions of $y$ with $l=2$ and $m=1$. Substitution into Equation (2.27) yields

$$
\operatorname{Res}_{x}(f, g)=\operatorname{det}\left[\begin{array}{ccc}
y-1 & 3 y-1 & 0 \\
-2 y+1 & 5 & 3 y-1 \\
y-1 & 0 & 5
\end{array}\right]=9 y^{3}+15 y^{2}+7 y-21
$$

The resultant is univariate in $y$ and the roots can be found to be $(0.824,-1.245-$ $1.131 I,-1.245+1.131 I)$. These are also the values of $y$ corresponding to the common roots of $f$ and $g$ and the intersection of the two polynomials. The corresponding values of $x$ are obtained by back substitution of each value of $y$ in turn into the original equations and solving for $x$. The values of $x$ corresponding to the set of $y$ values are $(-3.395,0.698-0.500 I, 0.698+0.500 I)$.

This technique can be expanded to larger sets of polynomials containing more variables. In such cases more elimination steps are required and the results must be validated in all of the polynomials. An example illustrates this. Consider the set of three polynomials

$$
\begin{aligned}
& f(x, y, z)=x^{2}-2 x y+y^{2}-z^{2} \\
& g(x, y, z)=x-3 y^{2}+y z-1 \\
& h(x, y, z)=x z-y z+3 z^{2} .
\end{aligned}
$$

Three resultants are required to obtain the univariate polynomial. Though the procedure may vary at this point, one possibility is to first use the resultant of $f$ and $g$ and the resultant of $g$ and $h$, both with respect to $z$ to obtain

$$
\begin{gathered}
\operatorname{Res}_{z}(f, g)=\left(2 y^{2}+x y+1-x\right)\left(-4 y^{2}+x y-1+x\right), \\
\operatorname{Res}_{z}(g, h)=-\left(x-3 y^{2}-1\right)\left(x y+8 y^{2}-3 x+3\right),
\end{gathered}
$$

which are both independent of $z$. The third resultant used is the resultant of $\operatorname{Res}_{z}(f, g)$ and 
$\operatorname{Res}_{z}(g, h)$ with respect to $y$ to obtain the univariate polynomial

$$
64(-1+x)^{4}\left(3 x^{2}-x+1\right)^{4}=0 .
$$

From this polynomial three solutions are obtained, each with multiplicity four, which are $x=1$ and $x=\frac{1 \pm \sqrt{11} I}{6}$. These three results must each be back substituted into the earlier equations. Substituting $x=1$ into $\operatorname{Res}_{z}(f, g)$ and $\operatorname{Res}_{z}(g, h)$ and solving for $y$ yields $y \in$ $\left\{-\frac{1}{2}, \frac{1}{4}, 0,0\right\}$ and $y \in\left\{\frac{1}{8}, 0,0,0\right\}$ respectively. $y=0$ is the only solution to both and upon substitution of $x=1$ and $y=0$ into $f, g$ and $h$ and solving for $z$ yields no common roots, thus $x=1$ is not part of a valid solution to the original system.

Similar back substitution of the remaining roots of Equation (2.28) yields the common solutions to $f, g$ and $h$ of

$$
\begin{aligned}
& x=\frac{1+\sqrt{11} I}{6}, \quad y=\frac{1+\sqrt{11}}{6}, \quad z=0 \text {, } \\
& x=\frac{1-\sqrt{11} I}{6}, \quad y=\frac{1-\sqrt{11}}{6}, \quad z=0 \text {. }
\end{aligned}
$$

This means that only the factor $\left(3 x^{2}-x+1\right)$ of Equation (2.28) yields accurate solutions and the other factors are artifacts of the method.

The resultant method of elimination is used in this thesis to find the solutions to sets of equations representing the intersection of constraint varieties. The polynomials of these equations may have more than three variables but the general procedure is the same. Care must always be taken to ensure only the accurate solutions are used.

\subsection{Gröbner Bases}

Gröbner Bases are used in this thesis to simplify sets of equations such that they are more manageable and to determine if there are redundant equations within a set. This section introduces Gröbner Bases and explores important characteristics and an algorithm for obtaining reduced Gröbner Bases. The required background information and algorithms for obtaining Gröbner Bases are presented by Adams and Loustaunau [21]. 


\subsubsection{Introduction to Gröbner Bases}

An ideal generated by $f_{1}=0, f_{2}=0, \ldots, f_{s}=0$, denoted by $\left\langle f_{1}=0, f_{2}=0, \ldots, f_{s}=0\right\rangle$ is defined as

$$
\left\langle f_{1}, f_{2}, \ldots, f_{s}\right\rangle=\left\{\sum_{i=1}^{n} u_{i} f_{i} \mid u_{i} \in k\left[x_{1}, \ldots, x_{n}\right], i=1, \ldots, s\right\} .
$$

It is defined that if $I=\left\langle f_{1}=0, f_{2}=0, \ldots, f_{s}=0\right\rangle$ is an ideal in $k\left[x_{1}, \ldots, x_{n}\right]$ then for $f, g \in I$ so does $f+g$, and for $f \in I$ and $h$, any polynomial in $k\left[x_{1}, \ldots, x_{n}\right], h f \in I$. The set $\left\{f_{1}, f_{2}, \ldots, f_{s}\right\}$ is called the generating set of $I$ and the purpose of obtaining the Gröbner Bases is to find a "better" generating set for the ideal. It is shown in [21] that $V(I)=V\left(f_{1}, f_{2}, \ldots, f_{s}\right)$, so for any generating set of $I$ the variety is unchanged. That being said, a "better" generating set of $I$ is one that allows for an understanding of the algebraic structure of $I=\left\langle f_{1}=0, f_{2}=0, \ldots, f_{s}=0\right\rangle$. Obtaining the Gröbner Bases provides this "better" generating set.

\subsubsection{Obtaining the Gröbner Bases}

For special sets of polynomials it may be easy to obtain a "better" generating set without using Gröbner Bases. These cases are outlined in [21] and include linear polynomials and polynomials in one variable. The methods used work only for these special cases and a more general method is required.

\section{Setting the Term Order}

For the algorithms for obtaining the Gröbner Bases to run smoothly some basic preparation is required. It is important that the generating set of polynomials used to construct $I$ have a standardized order of terms. With polynomials of one variable this is easy: the terms are ordered either from highest degree to lowest degree or vice versa. Linear polynomials are also simple because one must only pick the term order and remain consistent. For a general polynomial each term consists of a constant part and a power product part where the set 
of power products is given in [21] to be

$$
\mathbb{T}^{n}=\left\{x_{1}^{\beta_{i}} \cdots x_{n}^{\beta_{n}} \mid \beta_{i} \in \mathbb{N}, i=1, \ldots, n\right\}
$$

Another useful representation gives $x_{1}^{\beta_{i}} \cdots x_{n}^{\beta_{n}}$ as $\mathbf{x}^{\boldsymbol{\beta}}$, where $\boldsymbol{\beta}=\left(\beta_{1}, \ldots, \boldsymbol{\beta}_{n}\right) \in \mathbb{N}^{n}$.

It is possible to order the terms of a general polynomial in many different ways. The number of ways depending on the number of terms and degree of the polynomial. The degree of each term is the sum of $\beta_{i}, i=1, \ldots, n$ for that term and the degree of the polynomial is equal to that of the term with the maximum degree. When developing a logical term order it is useful to look ahead at what is required. Obtaining Gröbner Bases will require divison of polynomials and individual terms. If $\mathbf{x}^{\alpha}$, with $\alpha=\left(\alpha_{1}, \ldots, \alpha_{n}\right) \in \mathbb{N}^{n}$ is to divide $\mathrm{x}^{\boldsymbol{\beta}}$ then the term order should result in $\mathrm{x}^{\boldsymbol{\alpha}} \leq \mathrm{x}^{\boldsymbol{\beta}}$, which means $\alpha_{i} \leq \beta_{i}$ for all $i \in\{1, \ldots, n\}$. The term order must allow for the comparison of any two power products it must be possible to determine if $\mathbf{x}^{\boldsymbol{\alpha}}$ is greater than, less than or equal to $\mathbf{x}^{\boldsymbol{\beta}}$. One last condition that must be satisfied is that the terms are well-ordered, that is there is no infinite descending chain $\mathrm{x}^{\boldsymbol{\alpha}_{1}}>\mathrm{x}^{\boldsymbol{\alpha}_{2}}>\mathrm{x}^{\boldsymbol{\alpha}_{3}}>\cdots$. Using these requirements Adams and Loustaunau define three examples of term order.

The first is lexicographical order, abbreviated "lex", on $\mathbb{T}^{n}$ with $x_{1}>x_{2}>\cdots>x_{n}$. It is defined for this case that $\mathbf{x}^{\alpha}<\mathbf{x}^{\beta}$ if the first element $\alpha_{i}$ of $\boldsymbol{\alpha}$ from the left that is not equal to the equivalent element $\beta_{i}$ of $\boldsymbol{\beta}$ satisfies $\alpha_{i}<\beta_{i}$. In the case of two variables $x_{1}>x_{2}$ with order lex the term order is

$$
1<x_{2}<x_{2}^{2}<x_{2}^{3}<\cdots<x_{1}<x_{2} x_{1}<x_{2}^{2} x_{1}<\cdots<x_{1}^{2}<\cdots
$$

The second term order presented is degree lexicographical order, abbreviated "deglex", on $\mathbb{T}^{n}$ with $x_{1}>x_{2}>\cdots>x_{n}$. In this case it is defined that $\mathbf{x}^{\boldsymbol{\alpha}}<\mathbf{x} \boldsymbol{\beta}$ if $\sum_{i=1}^{n} \alpha_{i}<\sum_{i=1}^{n} \beta_{i}$ or in the case of $\sum_{i=1}^{n} \alpha_{i}=\sum_{i=1}^{n} \beta_{i}$ then $\mathbf{x}^{\boldsymbol{\alpha}}<\mathbf{x}^{\boldsymbol{\beta}}$ with respect to lex with $x_{1}>x_{2}>$ $\cdots>x_{n}$. In the case of two variables $x_{1}>x_{2}$ with order deglex the term order is

$$
1<x_{2}<x_{1}<x_{2}^{2}<x_{1} x_{2}<x_{1}^{2}<x_{2}^{3}<x_{1} x_{2}^{2}<x_{1}^{2} x_{2}<x_{1}^{3}<\cdots
$$


The final term ordering method presented by Adams and Loustaunau is degree reverse lexicographical order, abbreviated "degrevlex", on $\mathbb{T}$ with $x_{1}>x_{2}>\cdots>x_{n}$. It is defined for this case that $\mathbf{x}^{\boldsymbol{\alpha}}<\mathbf{x}^{\boldsymbol{\beta}}$ if $\sum_{i=1}^{n} \alpha_{i}<\sum_{i=1}^{n} \beta_{i}$ or in the case of $\sum_{i=1}^{n} \alpha_{i}=\sum_{i=1}^{n} \beta_{i}$ then $\mathrm{x}^{\alpha}<\mathrm{x}^{\beta}$ if the first element $\alpha_{i}$ of $\alpha$ from the right that is not equal to the equivalent element $\beta_{i}$ of $\boldsymbol{\beta}$ satisfies $\alpha_{i}>\beta_{i}$. With this definition the term order of the two variable case is no different from deglex, but when three or more variables are used then the difference becomes evident as is illustrated using three variables: with respect to deglex with $x_{1}>x_{2}>x_{3}$ the inequality $x_{1}^{2} x_{2} x_{3}>x_{1} x_{2}^{2}$ holds, however with respect to degrevlex with $x_{1}>x_{2}>x_{3}$ the inequality becomes $x_{1}^{2} x_{2} x_{3}<x_{1} x_{2}^{2}$.

In [21] Adams and Loustaunau prove that these three term orders satisfy the conditions described above, that is that if $\mathbf{x}^{\alpha}$ is to divide $\mathrm{x}^{\boldsymbol{\beta}}$ then the term order should result in $\mathbf{x}^{\boldsymbol{\alpha}} \leq \mathbf{x}^{\boldsymbol{\beta}}$, and that the term order is well-ordering.

After applying a legitimate term ordering algorithm such as one of the three just presented, a polynomial $f \in k\left[x_{1}, \ldots, x_{n}\right]$ may be written as

$$
f=a_{1} \mathbf{x}^{\alpha_{1}}+a_{2} \mathbf{x}^{\alpha_{2}}+\cdots+a_{r} \mathbf{x}^{\alpha_{r}}
$$

where $0 \neq a_{i} \in k, \mathbf{x}^{\boldsymbol{\alpha}_{i}} \in \mathbb{T}^{n}$ and $\mathrm{x}^{\boldsymbol{\alpha}_{1}}>\mathrm{x}^{\boldsymbol{\alpha}_{2}}>\cdots>\mathrm{x}^{\boldsymbol{\alpha}_{\boldsymbol{r}}}$. From this, the following functions are defined: the leading power product of $f, \operatorname{lp}(f)=\mathrm{x}^{\alpha_{1}}$; the leading coefficient of $f, \operatorname{lc}(f)=a_{1} ;$ and the leading term of $f, \operatorname{lt}(f)=a_{1} \mathbf{x}^{\alpha_{1}}$.

\section{Division of Polynomials}

To define Gröbner Bases it is necessary to have an algorithm for dividing polynomials. Such an algorithm is present in [21] and is summarized here.

For three polynomials $f, g$ and $h$ in $k\left[x_{1}, \ldots, x_{n}\right]$ with $g \neq 0$ the expression

$$
f \stackrel{g}{\longrightarrow} h
$$

means $f$ reduces to $h$ modulo $g$ if and only if $\operatorname{lp}(g)$ divides a non-zero term in $f$, called $X$ 
and

$$
h=f-\frac{X}{\operatorname{lt}(g)} g .
$$

The ability of $g$ to reduce polynomial $f$ may be dependant on the term order. The polynomial $h$ can be thought of as the remainder of the polynomial division. $f$ is fully reduced by $g$ when no term in $h$ is divisible by $\operatorname{lp}(g)$.

In the multivariate case it is possible to divide by more than one polynomial at a time. For polynomials $f, h$ and $f_{1}, \ldots, f_{s}$ in $k\left[x_{1}, \ldots, x_{n}\right]$ with $f_{i} \neq 0$ for all $i \in 1, \ldots, s$ and $F=\left\{f_{1}, \ldots, f_{s}\right\}$ the expression

$$
\stackrel{F}{\longrightarrow}+h
$$

means that $\mathrm{f}$ reduces to $\mathrm{h}$ modulo $\mathrm{F}$ if and only if there exists a sequence of indices $i_{1}, \ldots, i_{t} \in$ $\{1, \ldots, s\}$ and a sequence of polynomials $h_{1}, \ldots h_{t-1} \in k\left[x_{1}, \ldots, x_{n}\right]$ such that

$$
f \stackrel{f_{i_{1}}}{\longrightarrow} h_{1} \stackrel{f_{i_{2}}}{\longrightarrow} h_{2} \stackrel{f_{i_{3}}}{\longrightarrow} \cdots \stackrel{f_{i_{t-1}}}{\longrightarrow} h_{t-1} \stackrel{f_{i_{t}}}{\longrightarrow} h .
$$

A polynomial $r$ is said to be reduced with respect to $F$ if $r=0$ and no term in $r$ is divisible by any $\operatorname{lp}\left(f_{i}\right), i \in\{1, \ldots, s\}$ of $F$.

An algorithm for reducing a polynomial by division is given in [21] and is as follows. The goal of the algorithm is to find for a polynomial $f$ and a set of $s$ non-zero polynomials $F=\left\{f_{1}, \ldots, f_{s}\right\}$ the polynomials $u_{1}, \ldots, u_{s}$ and $r$ such that $f=u_{1} f_{1}+\cdots+u_{s} f_{s}+r$, where $r$ is reduced with respect to $F$ and $\max \left(\operatorname{lp}\left(u_{1}\right) \operatorname{lp}\left(f_{1}\right), \ldots, \operatorname{lp}\left(u_{s}\right) \operatorname{lp}\left(f_{s}\right), \operatorname{lp}(r)\right)=\operatorname{lp}(f)$. Note that a term order must be specified. The algorithm is initialized with $u_{i}=0$ for all $i \in\{1, \ldots, s\}, r=0$ and a polynomial $h=f$.

The algorithm then loops through the following statements until $h=0$, at which point $r$ is reduced with respect to $F$. If there is an index $i \in 1, \ldots, s$ such that $\operatorname{lp}\left(f_{i}\right)$ divides $\operatorname{lp}(h)$ then select the smallest $i$ that satisfies that condition and set

$$
u_{i}=u_{i}+\frac{\operatorname{lt}(h)}{\operatorname{lt}\left(f_{i}\right)}
$$


and

$$
h=h-\frac{\operatorname{lt}(h)}{\operatorname{lt}\left(f_{i}\right)} f_{i}
$$

Otherwise, if there is no such indices $i$ then set

$$
r=r+\operatorname{lt}(h)
$$

and

$$
h=h-\operatorname{lt}(h)
$$

There is some ambiguity in the division algorithm that depends on the ordering of the polynomials in $F$ since if the leading power product of more than one polynomial in $F$ divides $\operatorname{lp}(h)$ then the one with the smallest index is used. This will be addressed later using S-polynomials.

From the statement $f=u_{1} f_{1}+\cdots+u_{s} f_{s}+r$ it can be seen that $f-r \in\left\langle f_{1}, \ldots, f_{s}\right\rangle$, so if $r=0$ then $f \in\left\langle f_{1}, \ldots, f_{s}\right\rangle$, though the opposite is not always true. In cases where $r \neq 0$ but $f \in\left\langle f_{1}, \ldots, f_{s}\right\rangle$ it can be said that $f_{1}, \ldots, f_{s}$ is not the "best" generating set of the ideal. This is a situation where Gröbner bases are useful.

\section{Definition of Gröbner Bases}

A set of non-zero polynomials $G=\left\{g_{1}, \ldots, g_{t}\right\}$ in ideal $I$ is a Gröbner basis if all non-zero polynomials in $I$ can be reduced with respect to $G$.

To further describe characteristics of $G$ the following definition is required. The leading term ideal of a set of polynomials $S$ is called $\operatorname{lt}(S)$ and

$$
\operatorname{lt}(S)=\langle\operatorname{lt}(s) \mid s \in S\rangle
$$

That is, $\operatorname{lt}(S)$ is an ideal whose generating set is the leading terms of $S$.

It can now be said that the following four statements are equivalent. The proofs are provided in [21]. Note that $h \in k\left[x_{1}, \ldots, x_{n}\right]$. 
- $G$ is a Gröbner basis for $I$,

- $f \in I$ if and only if $f \stackrel{G}{\longrightarrow}+0$,

- $f \in I$ if and only if $f=\sum_{i=1}^{t} h_{i} g_{i}$ with $\operatorname{lp}(f)=\max \left(\operatorname{lp}\left(h_{i}\right) \operatorname{lp}\left(g_{i}\right)\right)$ for $i \in\{1, \ldots, t\}$, and

- $\operatorname{lt}(G)=\operatorname{lt}(I)$

In [21] it is also proven that $G$ is a generating set for $I$ and that every non-zero ideal $I$ has a Gröbner basis.

\section{Computing Gröbner Bases}

Before the algorithm for obtaining the Gröbner basis for an ideal is presented some definitions must be made. For two power products $X$ and $Y$ the least common multiple is the power product $L$ such that both $X$ and $Y$ divide $L$ and if another power product $Z$ is divisible by $X$ and $Y$, then $L$ always divides $Z$. This is denoted $L=\operatorname{lcm}(X, Y)$.

The S-polynomial of polynomials $f$ and $g$ is defined as

$$
S(f, g)=\frac{L}{\operatorname{lt}(f)} f-\frac{L}{\operatorname{lt}(g)} g
$$

where $L=\operatorname{lcm}(\operatorname{lp}(f), \operatorname{lp}(g))$. The S-polynomial can be used to cancel leading terms in a set of polynomials to eliminate the ambiguity of the polynomial division algorithm. This is shown in [21]. The S-polynomial is useful with regards to obtaining Gröbner bases because of Buchberger's Theorem which states that a non-zero set of polynomials $G=\left\{g_{1}, \ldots, g_{t}\right\} \in$ $k\left[x_{1}, \ldots, x_{n}\right]$ is a Gröbner basis for the ideal $I$ for which $G$ is a generating set if and only if for all indices $i \neq j$ where $i, j \in\{1, \ldots, t\}$

$$
S\left(g_{i}, g_{j}\right) \stackrel{G}{\longrightarrow}+0
$$

The proof of this theorem is provided in [21] and it is shown that the S-polynomial of two 
polynomials belongs to the ideal generated by those polynomials, that is

$$
S\left(f_{1}, f_{2}\right) \in\left\langle f_{1}, f_{2}\right\rangle
$$

Using Buchberger's theorem it is now possible to construct an algorithm to compute a Gröbner basis for an ideal. This is done by obtaining all possible S-polynomials from the generating set of the ideal and reducing with respect to the polynomials in the ideal. If the remainder of the division is not zero then the remainder is added to the generation set until all S-polynomials reduce to zero. The algorithm to do this is called Buchberger's algorithm, and the proof that this algorithm computes a Gröbner basis for an ideal is given in [21]. Buchberger's algorithm is as follows.

The input to the algorithm is a set of non-zero polynomials $F=\left\{f_{1}, \ldots, f_{s}\right\}$ and the intended output is a Gröbner basis $G$ for the ideal $\left\langle f_{1}, \ldots, f_{s}\right\rangle$. Initially $G$ is set equal to $F$ and a set of pairs of polynomials $\mathcal{G}$ is built such that $\mathcal{G}=\left\{\left\{f_{i}, f_{j}\right\} \mid f_{i} \neq f_{j} \in F\right\}$.

The algorithm then loops through the following until $\mathcal{G}=0$. A pair of polynomials $\{f, g\}$ is selected from $\mathcal{G}$ and eliminated from the set of polynomial pairs such that $\mathcal{G}=\mathcal{G}-\{\{f, g\}\}$. Buchberger's Theorem is now used to check if the current iteration of $G$ might be a Gröbner basis for $I$. This is done by reducing $S(f, g)$ with respect to $G$ and examining the remainder $h$, written mathematically as

$$
S(f, g) \stackrel{G}{\longrightarrow}+h .
$$

If $h=0$ then $G$ might be a Gröbner basis for $I$ (depending on the outcome of subsequent passes through the loop,) otherwise if $h \neq 0$ then $h$ must be added to $G$ and the set of $\mathcal{G}$ must be updated to include the new sets of polynomial pairs that can be made using $h$ and the polynomials in $G$, written mathematically in the proper programming logic order

$$
\mathcal{G}=\mathcal{G} \cup\{\{u, h\} \forall u \in G\}
$$

then

$$
G=G \cup\{h\}
$$


Upon completing this loop $G$ will be a Gröbner basis for $I$.

The result of Buchberger's Algorithm is not necessarily unique because the order of the polynomials in $F$ may vary, as could the ordering of the polynomial pairs in $\mathcal{G}$. The computed Gröbner basis is also dependant on the term order. To obtain a unique Gröbner basis for any ideal $I$ minimal and reduced Gröbner bases are used.

A minimal Gröbner basis $G=\left\{g_{1}, \ldots, g_{t}\right\}$ is one where all $\operatorname{lc}\left(g_{i}\right)=1$ and $\operatorname{lp}\left(g_{i}\right)$ does not divide $\operatorname{lp}\left(g_{j}\right)$ for all $i \neq j \in\{1, \ldots, t\}$. A minimal Gröbner basis is found by eliminating all $g_{i}$ that has $\operatorname{lp}\left(g_{i}\right)$ divisible by another $\operatorname{lp}\left(g_{j}\right)$ for $i \neq j$ and dividing the each of polynomials $g_{i}$ in the remaining set by $\operatorname{lc}\left(g_{i}\right)$ for $i$ from 1 to the number of polynomials in the resulting set. Minimal Gröbner bases are not unique, however all minimal Gröbner bases for a given ideal have the same number of terms and if reordered properly, the same leading terms. The proof of this is provided in [21].

To provide a unique Gröbner basis for any ideal (proven in [21]) reduced Gröbner bases are used. A reduced Gröbner basis $G=\left\{g_{1}, \ldots, g_{t}\right\}$ has for all $i \in\{1, \ldots, t\}, \operatorname{lc}\left(g_{i}\right)=1$ and for all polynomials $g_{i}$ in $G$ no term is divisible by the leading power product of any other polynomial in $G$. Another way of stating this is that for all $i \in\{1, \ldots, t\}, g_{i}$ is reduced with respect to $G-\left\{g_{i}\right\}$.

The reduced Gröbner basis for ideal $I$ is obtained by starting with a minimal Gröbner basis $G=\left\{g_{1}, \ldots, g_{t}\right\}$ and using the following reduction process:

$$
\begin{aligned}
& g_{1} \stackrel{H_{1}}{\longrightarrow}+h_{1}, \text { where } H_{1}=\left\{g_{2}, \ldots, g_{t}\right\} \\
& g_{2} \stackrel{H_{2}}{\longrightarrow}+h_{2}, \text { where } H_{2}=\left\{h_{1}, g_{3}, \ldots, g_{t}\right\} \\
& g_{3} \stackrel{H_{3}}{\longrightarrow}+h_{3}, \text { where } H_{3}=\left\{h_{1}, h_{2}, g_{4}, \ldots, g_{t}\right\} \\
& \vdots \\
& g_{t} \stackrel{H_{t}}{\longrightarrow}+h_{t}, \text { where } H_{t}=\left\{h_{1}, h_{2}, h_{3}, \ldots, h_{t-1}\right\} .
\end{aligned}
$$

The set of polynomials $H=\left\{h_{1}, \ldots, h_{t}\right\}$ is the unique Gröbner basis for $I$. The proof of this is provided in [21].

The Gröbner basis provides a simpler generating set for a given ideal. The variety of 
the Gröbner basis is the same as for a more complex generating set and allows for easier computations and a better understanding of the geometry of the problem which may allow for simple solutions.

\subsection{Polynomial Homotopy and Continuation Methods}

In Section 2.9 elimination of variables using resultants is presented as a way to algebraically solve a system of polynomials. In some situations, such as systems with very large polynomials like those with many variables or of high total degree, this algebraic method becomes impractical because the resultants become even larger equations. In such situations computer algebraic software may not be capable of performing the analysis, therefore a numerical method is often required to find the solution to the set of polynomials. Using polynomial homotopy continuation methods one can numerically find all the solutions to a set of equations. This section provides a brief introduction to polynomial homotopy continuation methods, the interested reader is referred to Sommese and Wampler [22], $\mathrm{Li}$ [23] and Raghavan and Roth [24].

The degree of each term of a polynomial is equal the sum of the exponents on the variables in that term and the degree of a polynomial is equal to that of the term with the maximum degree in the polynomial. In a system of polynomials the total degree is the product of the degrees of all polynomials in the system. Bezout's theorem states that the Bezout number is equal to the total degree of a system of polynomials and that the number of solutions to the system is not greater than the Bezout number.

\subsubsection{Introduction to Continuation Methods}

The idea behind homotopy continuation is as follows. For a system $P(x)$ of $n$ polynomials

$$
\begin{array}{cl}
p_{1}\left(x_{1}, \ldots, x_{n}\right) & =0 \\
& \vdots \\
p_{n}\left(x_{1}, \ldots, x_{n}\right) & =0
\end{array}
$$


with $n$ unknown variables $x_{i}$ for $i \in\{1, \ldots, n\}$ it is desired to find the set of all solutions that satisfy all polynomials. To do this one starts by selecting a suitable simple system of equations, $Q(x)$, with known or easily obtained solutions that has the same number of solutions as $P(x)$. Utilizing the fact that small changes in the parameters of a system cause small changes in the numerical value of the solutions to the system, the known solutions are used to work towards the solutions of $P(x)$ by gradually modifying the coefficients of $Q(x)$, numerically tracking the change to each root until the roots of $P(x)$ are obtained. The parallel nature of this method, that is that following the path taken by each root from the starting solution to the finial solution needs no knowledge of the other solutions, means that this method is well suited to computation on parallel or separate processors because each path can be analyzed independently.

The most basic method of tracking solutions uses the set of equations

$$
H(x, t)=(1-t) Q(x)+t P(x)=0
$$

where $t$ varies from 0 to 1 . When $t=0$, then $H(x, 0)=Q(x)$, the starting system with known solutions and when $t=1$, then $H(x, 1)=P(x)$, the system for which the roots are desired. For a correctly chosen $Q(x)$ each root can be followed as $t$ increments from 0 to 1 , this will be discussed later. The first step is to select a start system $Q(X)$.

\subsubsection{Selecting a Start System}

$\mathrm{Li}$ [23] proposes that the start system must posses three important properties: triviality, smoothness, and accessibility. Triviality refers to the fact that the solutions to $Q(x)=0$ must be known, smoothness requires that for $H(x, t)=0$ for $0 \leq t<1$ the solution set consists of a finite number of smooth paths parameterized by $t$, and accessibility means that every solution to the system $H(x, 1)=P(x)$ is achieved by a path originating from $H(x, 0)=Q(x)$. 
A typical choice for the start system $Q(x)$ for the system of $n$ polynomials $P(x)$ is

$$
q_{i}=a_{i} x_{i}^{d_{i}}-b_{i}
$$

where $i \in\{1, \ldots, n\}, d_{i}$ is the degree of $p_{i}$ and the constants $a_{i}$ and $b_{i}$ are assigned random non-zero values $\in \mathbb{C}$. Using this start system guarantees that the Bezout number of $Q(x)$ is equal to that of $P(x)$. This starting system satisfies all three properties of a good start system listed above: triviality, smoothness and accessibility. With this being said one could continue with continuation methods to find the solutions to $P(x)$, however this may be be prohibitively inefficient. The reason for this is that $P(x)$ may have fewer independent solutions then the Bezout number suggests. Such a system is called deficient. The number of starting solutions for $Q(x)$ built using Equation (2.30) is equal to the Bezout number, however if $P(x)$ is deficient the number of finite solutions of $P(x)$ is less than that of $Q(x)$ and some of the solution paths will diverge to infinity. These divergent paths are called extraneous and result in wasted computing power. It is therefore desirable to reduce the number of paths while maintaining triviality, smoothness and especially important when reducing the number of start solutions and paths, accessibility.

One method of reducing the number of starting solutions involves multi-homogeneous (m-homogenous) systems and the m-homogeneous Bezout number. For the polynomials $p_{1}, \ldots, p_{n}$ of system $P(x)$ the unknowns $x_{1}, \ldots, x_{n}$ may be divided into $m$ groups $\left\{x_{11}, \ldots, x_{1 k_{1}}\right\}, \ldots,\left\{x_{m 1}, \ldots, x_{1 k_{m}}\right\}$, where $k_{i}$ is the number of elements in group $i$ with $i \in\{1, \ldots, m\}$ and $k_{1}+\ldots+k_{m}=n$. Depending on the size of the system in question there are many possible groupings of the unknowns. For each polynomial $l, l \in\{1, \ldots, n\}$ the degree with respect to the variables of group $j, j \in\{1, \ldots, m\}$ is assigned to $D_{j l}$. The m-homogeneous Bezout number is equal to the coefficient of $\prod_{j=1}^{m} \alpha_{j}^{k_{j}}$ in the product $\prod_{l=1}^{n}\left(\sum_{j=1}^{m} d_{j l} \alpha_{j}\right)$. The m-homogeneous Bezout number provides a better upper bound on the number of finite solutions to a system of polynomials. Changing the grouping of unknowns may have an effect on the m-homogeneous Bezout number. A simple example from Raghavan and Roth [24] helps illustrate how to obtain the m-homogeneous Bezout number. 
Consider the system of polynomials in the unknowns $x$ and $y$ with constant coefficients

$$
\begin{aligned}
& f_{1}: a_{11} x y+a_{12} x+a_{13}=0 \\
& f_{2}: a_{21} x y+a_{22} x+a_{23}=0 .
\end{aligned}
$$

The degree of both polynomials is 2 , so the Bezout number of this system is $2 \times 2=4$. There are two possible groupings of the unknowns: one group $\{x, y\}$ or two groups $\{x\}$ and $\{y\}$, for this example the second option of two groups is used, therefore $m=2$ and $k_{1}=k_{2}=1$. Substitution into the product $\prod_{l=1}^{n}\left(\sum_{j=1}^{m} d_{j l} \alpha_{j}\right)$ yields $\left(\alpha_{1}+\alpha_{2}\right)\left(\alpha_{1}+\alpha_{2}\right)$ and the $\mathrm{m}$-homogeneous number is the coefficient of $\prod_{j=1}^{m} \alpha_{j}^{k_{j}}=\alpha_{1}^{2} \alpha_{2}^{2}$ which in this case is 2 . As can be seen the $\mathrm{m}$-homogeneous Bezout number less than the Bezout number and provides a bound on the number of solution that is less than the Bezout number and helps to reduce the estimate on the number of finite roots of a system, giving the basis for a better starting system $Q(x)$ leading fewer extraneous paths and greater computational efficiency.

The start system $Q(X)$ for an m-homogeneous system must have the same $\mathrm{m}$ homogeneous form and m-homogeneous Bezout number as $P(x)$. Raghavan and Roth [24] suggest that this can be done by assembling a set of $n$ polynomials $q_{i}(x), i \in\{1, \ldots, n\}$, where each is a product of factors given by $\prod_{j=1}^{m} p_{j l}\left(x_{j 1}, \ldots, x j k\right)=0$, and the degree of $p_{j l}$ is equal to that of the corresponding equation $p_{i}(x)$. From the example above the factor corresponding to the group $\{x\}$ may be of the form $(a x-b)$, where $a$ and $b$ are random non-zero complex numbers.

Selecting appropriate and efficient starting systems is an area of active research and the interested reader is referred to the authors listed at the start of this section for more insight.

\subsubsection{Projective Transformation}

In order to successfully track solutions at infinity a projective transformation is used. Raghavan and Roth [24] provide a concise summary of this topic while Sommese and Wampler [22] discuss the topic in more detail.

For each variable group of the m-homogeneous grouping $x_{i j}$ is replaced with 
$\frac{x_{i j}}{y_{i 0}}$ for $i \in\{1, \ldots, m\}$ and $j \in\left\{1, \ldots, k_{m}\right\}$ such that the groups become $\left\{x_{11}, \ldots, x_{1 k_{1}}, y_{10}\right\}, \ldots,\left\{x_{m 1}, \ldots, x_{m k_{m}}, y_{m 0}\right\}$ and there are now $n+m$ unknowns in a system of $n$ polynomials. The fact that the new system is indeterminate is resolved by introducing linear equations for each homogeneous group. The equations are of the form

$$
c_{i 0} y_{i 0}+c_{i 1} x_{i 1}+\cdots+c_{i k_{m}} x_{i k_{m}}=0
$$

for $i \in\{1, \ldots, m\}$, where the coefficients $c_{i, j}$ are random non-zero complex numbers. This adds additional equations to the set and the starting system must be adjusted accordingly. At the conclusion of the path tracking if $y_{i 0}=0$ then the corresponding solution of $P(x)$ at the end of the path is at infinity. Conversely, if $y_{i 0}$ is finite then the corresponding solution at the end of the path is finite and is given by $\frac{x_{i j}}{y_{i 0}}$ for $i \in\left\{1, \ldots, k_{m}\right\}$.

\subsubsection{Path Following}

With the start system $Q(x)$ and its solutions identified and the corresponding $H(x, t)$ of Equation (2.29) assembled it is time to start the path tracking of the polynomial continuation method. This is done by using the known solutions to $Q(x)$ as starting points to numerically obtaining the solution to $H(x, t)$ with $t$ equal to some small step from zero towards one. The new solutions are iteratively used as starting estimates as $t$ is slowly incrementing from $t=0$ towards $t=1$. At $t=1$ the solutions for the system $P(x)$ are obtained.

A path tracking algorithm provided by Wampler, Morgan and Sommese [25] is summarized here. A known solution to $H(x, 0)$ is assigned to $\left(x_{0}, t_{0}\right)$, where $x_{0}$ is one of the known solutions to $H(x, 0)$ for $t=t_{0}$. The value $t$ is then increased by some small, predetermined value $\Delta t$ and the values of $x_{0}$ are used as an prediction of the solution of $H\left(x, t_{0}+\Delta t\right)$. Newton's method is used to correct the prediction of the solution $x$. The application of this method requires the Taylor expansion for $\mathrm{H}(\mathrm{x}, \mathrm{t})$ with small increments of $\Delta x$ and $\Delta t$ which is

$$
H(x+\Delta x, t+\Delta t) \approx H(x, t)+J_{x} \Delta x+J_{t} \Delta t
$$


where $J_{x}$ and $J_{t}$ are the Jacobians of $H(x, t)$ with respect to $x$ and $t$ respectively. Setting the results of Equation (2.33) equal to zero and rearranging to get the correction to the prediction, $\Delta x$, by

$$
\Delta y=-J_{y}^{-1} J_{t} \Delta t
$$

Because of the approximations used, it is possible to set $t=t_{0}+\Delta t$ and repeat the correction several times until an acceptable error is achieved. At that point $t$ is again incremented by $\Delta t$ and the procedure starts over. This is repeated until $t=1$ and the resulting solution is one of the solutions to $H(x, 1)=P(x)$. The entire iterative path following procedure must be performed for every starting solution of $Q(x)$. A path tracker may make the process more efficient by halving $\Delta t$ if too many corrections are required at each step to achieve the desired accuracy or increasing $\Delta t$ if very few corrections are required. The fact that each path may be followed independently of the others is what makes this method attractive for computation on parallel processors or separate processors.

Morgan and Sommese [26] provide numerical examples of polynomial homotopy continuation for geometric modeling, chemical equilibrium and mechanism kinematics applications that help illustrate the technique. Of particular interest to this thesis are the geometric modeling example which obtains the intersection of two quadric surfaces and a plane, and the mechanism problem which is a numerical solution to the inverse kinematics problem.

The work presented in this thesis that relates to polynomial homotopy continuation was performed using software called PHCpack. A complete description of this software as well as instructions on how to obtain it are provided by Verschelde [27]. 


\section{Chapter 3}

\section{Review of Previous Research}

This chapter presents a review of the research that is the basis for the original work presented in this thesis. The first part of this chapter introduces the Griffis-Duffy Platform (GDP) and the characteristics of GDPs that are relevant to this thesis. Focus is given to the special configuration of GDP used later in this thesis and the phenomenon of self-motions. The second section of this chapter examines techniques for solving the inverse kinematics problem for serial manipulators, and more specifically $6 \mathrm{R}$-manipulators. A brief overview of some historical methods for solving the inverse kinematics is provided followed by a more detailed look at a novel method introduced by Pfurner [2] that utilizes kinematic mapping.

\subsection{Overview of Griffis-Duffy Platforms}

The GDP is a special configuration of the six-legged Stewart-Gough platform patented by Griffis and Duffy in 1993 [28]. This section defines the GDP and describes the special configuration of GDP used in this thesis, followed by a discussion of the self-motion phenomenon and how it affects the special GDP configuration.

\subsubsection{Description of GDP and Possible Configurations}

The GDP is a Stewart-Gough platform characterized by a planar fixed base and planar moving platform each with six specially placed spherical joint anchor points for the six legs of the manipulator. Six anchor points lie on the perimeter of a triangle on each of the fixed 
base and moving platform. Six anchor points are located on each of the vertices of the two triangles and the remaining six being located one on each edge such that each leg has one anchor point on the fixed base and one anchor point on the moving platform.

The patent by Griffis and Duffy proposes controlling the pose of the platform by actively changing the length of each leg using linear actuators. As will be shown, controlling the leg lengths may not provide for full control of the EE pose for all GDPs because many anchor point architectures lead to the phenomenon of self-motion, which means the moving platform can move relative to the fixed base in a way that is not controllable by actuating the leg lengths. It is these self-motions which are exploited in this thesis, not the changing of leg lengths.

There are many different GDP architecture configurations that are possible with different combinations of edge and vertex connections between the fixed base and moving platform. One additional common condition, though not necessary, is that each edge connection be on the midpoints of the line segments between vertices of the triangle. With this condition there are still many possible configurations, but two important groups are vertex-to-vertex and midline-to-vertex. In the vertex-to-vertex configuration, shown in Figure 3.1, a leg with a vertex anchor point on the base has an vertex anchor point on the platform, with the order of the leg end points around the perimeters of the triangles being the same on both the fixed base and moving platform. The midline anchor points are between the same legs on both the fixed base and moving platform. The midline-to-vertex configuration, shown in Figure 3.2 , connects a vertex anchor point on the fixed base with a midline anchor point on the moving platform and vice versa, maintaining the same order of legs around the perimeter of the fixed base and moving platform.

Throughout this thesis the midline-to-vertex configuration will be used with the added conditions that the fixed base and moving platform anchor points form congruent equilateral triangles. The midline-to-vertex configuration is subject to self-motions, as is shown in Section 3.1.2. This configuration is used because, as will be shown, the self-motions of the platform are simple and the moving platform remains parallel to the fixed base throughout the self-motions when the legs are of equal length. 


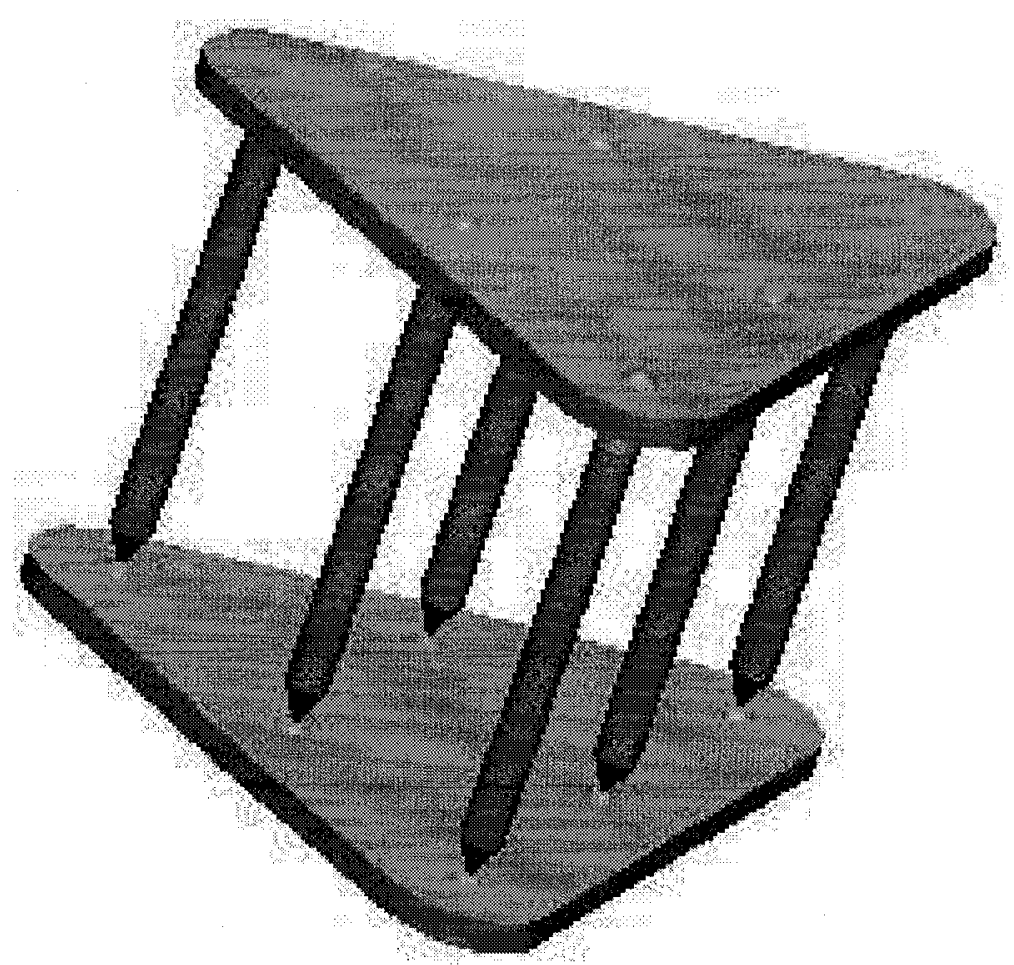

Figure 3.1: Example of the vertex-to-vertex configuration of GDP.

\subsubsection{Self-motions of GDPs}

In [29], Husty and Karger examine self-motions of GDPs. The term self-motion refers to the ability of the EE of a mechanism to move without actuator input. In the GDP this means that the moving platform can move relative to the fixed base without joint actuation. In other words, without changing the length of the legs. Husty and Karger's procedure for obtaining the self-motions of a GDP is summarized here. Discussions on the self-motions of all Stewart-Gough platforms are provided buy Karger and Husty [30] and Husty and Karger [31].

Husty shows in [9] that a set of seven quadratic equations governs the direct kinematics for all Stewart-Gough platforms. One of the equations is $S_{6}^{2}$, Equation (2.12), and the 


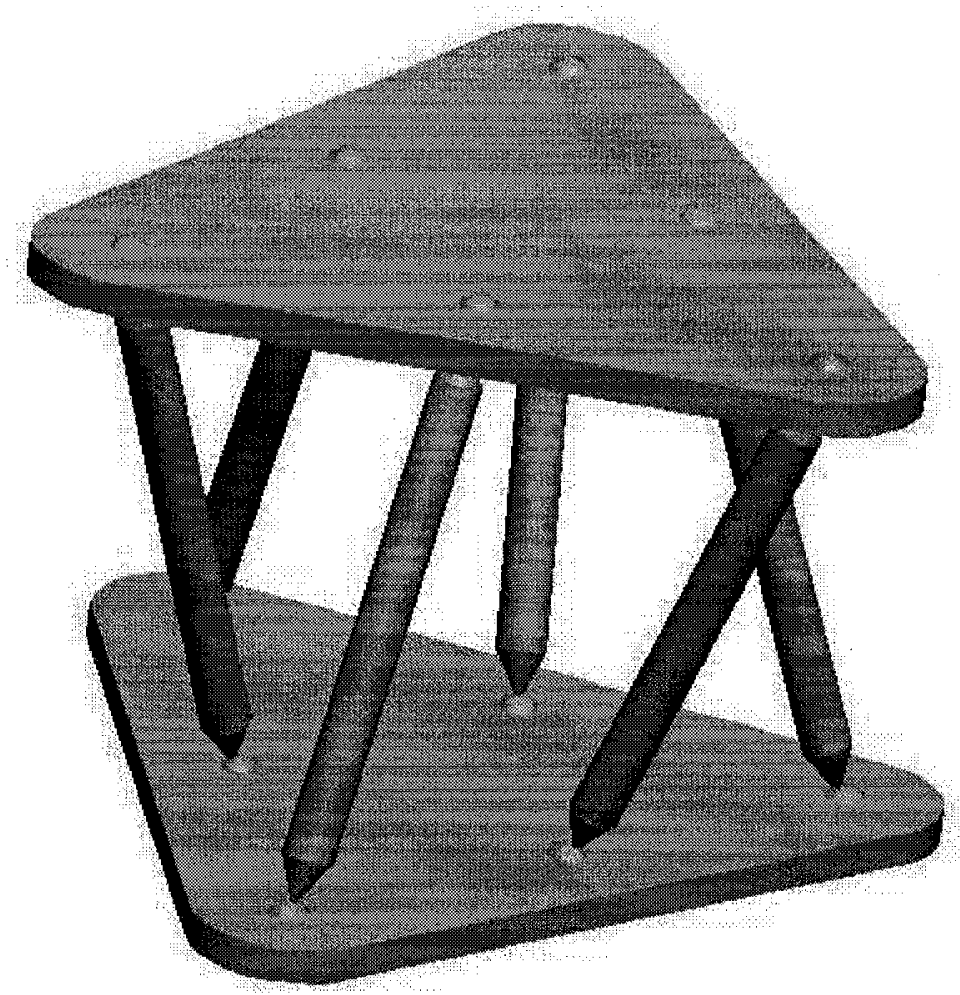

Figure 3.2: Example of the midline-to-vertex configuration of GDP.

remaining six are of the form

$$
\begin{aligned}
h_{i}= & R_{i}\left(x_{0}^{2}+x_{1}^{2}+x_{2}^{2}+x_{3}^{2}\right)+4\left(y_{0}^{2}+y_{1}^{2}+y_{2}^{2}+y_{3}^{2}\right)-2 x_{0}^{2}(A a+B b+C c)+ \\
& 2 x_{1}^{2}(-A a+B b+C c)+2 x_{2}^{2}(A a-B b-C c)+2 x_{3}^{2}(A a+B b+C c)+ \\
& 2 x_{3}^{2}(A a+b b-C c)+4\left(x_{0} x_{1}(B c-C b)+x_{0} x_{2}(C a-A c)+x_{0} x_{3}(A b-B a)-\right. \\
& x_{1} x_{2}(A b+B a)-x_{1} x_{3}(A c+C a)-x_{2} x_{3}(B c+C b)+\left(x_{0} y_{1}-y_{0} x_{1}\right)(A-a)+ \\
& \left(x_{0} y_{2}-y_{0} x_{2}\right)(B-b)+\left(x_{0} y_{3}-y_{0} x_{3}\right)(C-c)+\left(x_{1} y_{2}-y_{1} x_{2}\right)(C-c)- \\
& \left.\left(x_{1} y_{3}-y_{1} x_{3}\right)(B-b)+\left(x_{2} y_{3}-y_{2} x_{3}\right)(A-a)\right)=0,
\end{aligned}
$$

with $i=1, \ldots 6$. The terms $(a, b, c)$ are the coordinates of the anchor point for leg $i$ moving with the moving platform of the manipulator in a reference frame fixed to the moving platform, $(A, B, C)$ are the coordinates of the corresponding anchor point for leg $i$ on the fixed base of the manipulator in a reference frame affixed to the fixed base, and 
$R_{i}=A^{2}+B^{2}+C^{2}+a^{2}+b^{2}+c^{2}+r_{i}^{2}$, with $r_{i}, i=1, \ldots, 6$ being the joint parameter or length of leg $i . x_{j}$ and $y_{j}, j \in\{0, \ldots 3\}$ are the Study parameters. It is convenient to assign the base and platform reference frames such that the equations are as simple as possible.

For general Stewart-Gough type manipulators the solution to the set of seven quadratic equations is a set of discrete points on $S_{6}^{2}$. There are however platform configurations including, as Husty and Karger show, many GDP configurations where the solution variety is a curve or surface on $S_{6}^{2}$, meaning that the platform can move independent of the joint parameters which are assigned fixed values for a particular pose. In this thesis the additional constraints that the fixed base and moving platform are congruent meaning $p=q$ in Figure 3.3 and that all legs are of the same length means $r_{i}=r$ for all $i \in\{1, \ldots, 6\}$.

In [29] Husty and Karger use the midline-to-vertex configuration with the coordinates given in Table 3.1. The coordinate systems are shown in Figure 3.3, wherein variables $p$

Table 3.1: Coordinates of anchor points for special midline-to-vertex configuration.

\begin{tabular}{|c||c|c|c||c||c|c|c|}
\hline & $A$ & $B$ & $C$ & & $a$ & $b$ & $c$ \\
\hline$P_{1}$ & $-p$ & 0 & 0 & $p_{1}$ & $\frac{q}{2}$ & $\frac{q \sqrt{3}}{2}$ & 0 \\
\hline$P_{2}$ & 0 & 0 & 0 & $p_{2}$ & 0 & $q \sqrt{3}$ & 0 \\
\hline$P_{3}$ & $p$ & 0 & 0 & $p_{3}$ & $-\frac{q}{2}$ & $\frac{q \sqrt{3}}{2}$ & 0 \\
\hline$P_{4}$ & $\frac{p}{2}$ & $\frac{p \sqrt{3}}{2}$ & 0 & $p_{4}$ & $-q$ & 0 & 0 \\
\hline$P_{5}$ & 0 & $p \sqrt{3}$ & 0 & $p_{5}$ & 0 & 0 & 0 \\
\hline$P_{6}$ & $-\frac{p}{2}$ & $\frac{p \sqrt{3}}{2}$ & 0 & $p_{6}$ & $q$ & 0 & 0 \\
\hline
\end{tabular}

and $q$ are defined. With these coordinates, the six constraint equations $h_{i}, i=1, \ldots, 6$ can be constructed by substituting the values from Table 3.1 into Equation (3.1) for each leg. The seventh constraint equation is the Study quadric $S_{6}^{2}$. A set of five difference equations, $U_{i}, i=1, \ldots, 5$, that are linear in the terms $y_{0}, y_{1}, y_{2}$ and $y_{3}$ is constructed where $U_{1}=h_{1}-h_{3}, U_{2}=h_{2}-h_{5}, U_{3}=h_{4}-h_{6}, U_{4}=h_{1}-h_{2}$ and $U_{5}=h_{1}-h_{4}$. The motion of interest is where all points on the platform move on a spherical path. This is called Borel-Bricard motion and is discussed by Bottema and Roth [15]. Such motions lead to the situation where $x_{1}=x_{2}=0$. Three of the difference equations are used to construct a system that can be solved for four of the $y_{i}$ terms. The remaining Study parameters can, 


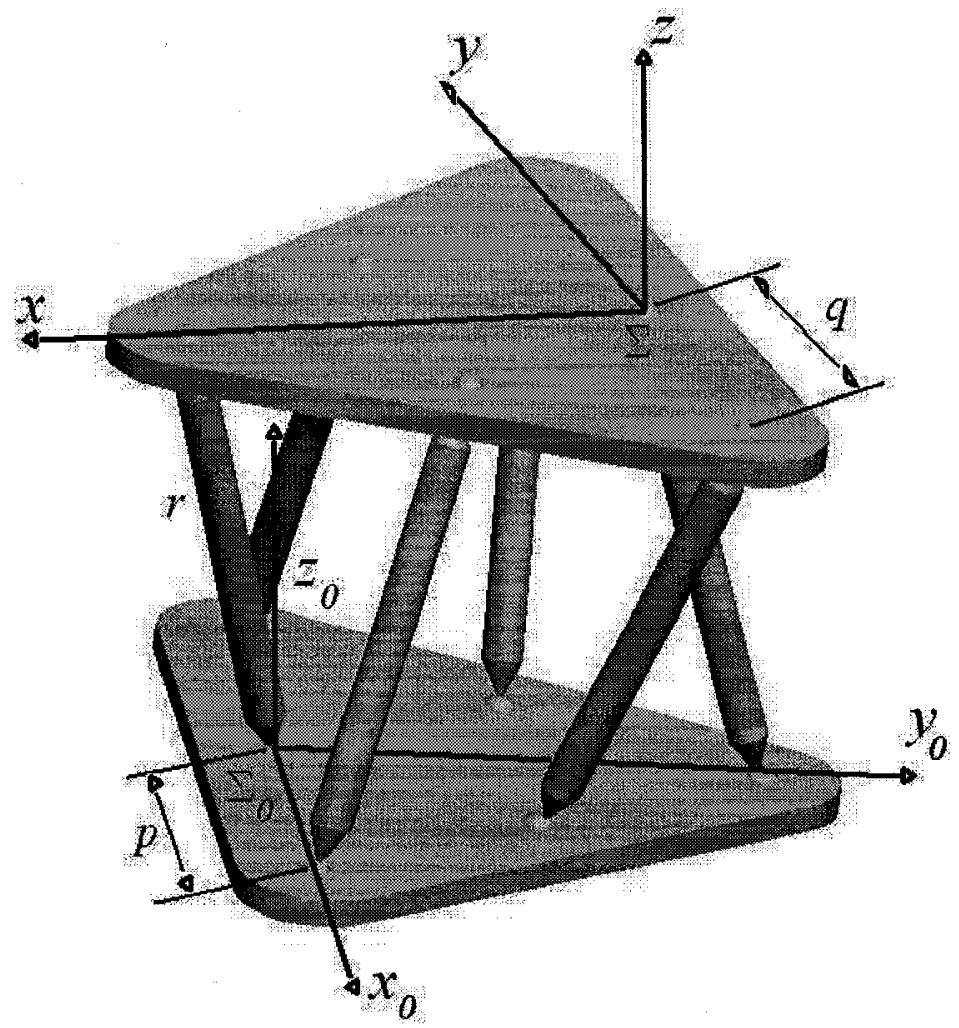

Figure 3.3: Midline-to-vertex GDP with coordinate systems and variables defined.

without loss in generality, be set such that $x_{0}=\cos (t)$ and $x_{3}=\sin (t)$ to yield the motion

$$
\mathbf{Q}=\left[\begin{array}{cccc}
1 & 0 & 0 & 0 \\
\frac{2}{3} q \sqrt{3} \sin (t) \cos (t) & \cos (2 t) & -\sin (2 t) & 0 \\
\frac{2}{3} q \sqrt{3} \sin ^{2}(t) & \sin (2 t) & \cos (2 t) & 0 \\
\rho \cos (t) & 0 & 0 & 1
\end{array}\right]
$$

where $\rho$ is a function of the leg length $r$, and $t$ is the rotation of the moving platform frame relative to the fixed base frame about the shared $Z$-axis. The transformation from the platform coordinate system with position vectors $\mathbf{x}$ to the base coordinate system with position vectors $\mathbf{x}_{0}$ is given by

$$
\mathbf{x}_{0}=\mathbf{Q} \mathbf{x}
$$

Of interest in this thesis is the change in distance between the base and platform. From $\mathbf{Q}$ 
it can be shown that a point at the geometric centre of the platform triangle moves on a line perpendicular to the plane of the platform, where the motion is characterized by

$$
\mathbf{Q}=\left[\begin{array}{cccc}
1 & 0 & 0 & 0 \\
0 & \cos (2 t) & -\sin (2 t) & 0 \\
0 & \sin (2 t) & \cos (2 t) & 0 \\
\rho \cos (t) & 0 & 0 & 1
\end{array}\right]
$$

It is to be seen that when $t=0$ the distance between the base and platform is $\rho$. Another possible zero position that differs from the one presented by Husty and Karger is where the zero position is shifted to the theoretical position where the fixed base and moving platform lie on top of each other. In this position the rotation angle, now called $\theta$, between the fixed base and moving platform is zero. This position is not obtainable in reality because it requires that the rigid fixed base and moving platform of the GDP pass through each other, but it is useful for theoretical analysis. $\theta$ refers to the rotation angle about the shared $Z$-axis of the frame affixed to the moving platform relative to the frame affixed after the new zero position is established. After shifting the zero position of the platform and repositioning the moving platform coordinate system such that it is coincident with the fixed base coordinate system in the new zero position, which can be done without loss in generality, the motion of the point at the geometric centre of the platform triangle is now given by

$$
\mathbf{Q}=\left[\begin{array}{cccc}
1 & 0 & 0 & 0 \\
0 & \cos (2 \theta) & -\sin (2 \theta) & 0 \\
0 & \sin (2 \theta) & \cos (2 \theta) & 0 \\
\rho \sin (\theta) & 0 & 0 & 1
\end{array}\right]
$$

and the distance between the base and platform is sinusoidal. For the purposes of this thesis the leg length variable is set to unity $(\rho=1)$. Using a different leg length would change the constants in the equations, but the overall theory is the same. 


\subsection{Inverse Kinematics of 6R-Manipulators}

Unlike the method for obtaining the direct kinematic equations of a serial manipulator presented in Section 2.5.2, the solution to the inverse kinematic problem for serial manipulators is, in many cases, very complicated. The complete solution to the inverse kinematic problem provides all possible sets of joint variables that place the EE of a manipulator in a desired pose. Historically there are many methods for solving this problem utilizing different techniques including both numerical and algebraic methods. Many of the methods focus on a specific type of serial manipulator, such as one with intersecting joint axes.

Of particular interest are $6 \mathrm{R}$-manipulators because of their relative simplicity while maintaining, in general (but not always), 6DOF and a large reachable workspace. Such manipulators are commonly used for industrial applications and have therefore been the focus of study for many researchers. Some examples of applying inverse kinematics methods to industrial 6R-manipulators include Chen and Parker [32] who use a numerical approach to the inverse kinematics to aid in the calibration of a PUMA 560 industrial $6 \mathrm{R}$ robotic manipulator; Lloyd and Hayward [33] use symbolic algebra to set up solutions for special configurations of $6 \mathrm{R}$ industrial robots; Manseur and Doty [34] present algorithms to improve the efficiency of the solution to the inverse kinematics problem for industrial applications of 6R-manipulators; Pashkevich [35] implements an algorithm for the inverse kinematics of industrial manipulators with offset wrists; and Chapelle and Bidaud [36] use analytical methods on kinematic models of PUMA 560 and GMF Arc Mate industrial 6R-manipulators.

This section provides an overview of some methods that have been developed and then focuses specifically on a technique utilizing kinematic mapping developed by Pfurner [2].

\subsubsection{Overview of Some Methods of Solving the Inverse Kinematics Prob- lem}

The inverse kinematics problem is well known and because of the usefulness of $6 \mathrm{R}$ manipulators in industry there has been extensive research in this area. It is not feasible to 
explore all of the methods and publications that exist, but it is important to look at some major milestones and well known techniques. Using the results of the direct kinematics as presented in Section 2.5.2, evaluating Equation (2.18) yields 12 equations in six unknown joint variables. Many of the methods aim to reduce the number of equations or determine the exact number of solutions. This section gives an overview of major milestones in solving the inverse kinematics problem. A detailed history may be found in [2].

In 1968 Pieper [37] explored 6DOF manipulators, focusing on those with three consecutive intersecting axes. His methods apply to many manipulators with R- and P-pairs and is often applied to many existing industrial robots which, because of the intersecting axes limitation, leads to many being wrist-partitioned. The method for applying Pieper's method to $6 \mathrm{R}$-manipulators is provided in [14].

Roth et al. [38] in 1973 showed that there were at most 32 solutions to the problem. Duffy and Crane [39] in 1980 provided a method for obtaining a polynomial of degree 32, though some of the roots did not provide solutions to the inverse kinematics problem. In 1985 Tsai and Morgen [40] used homotopy continuation methods to solve the problem and obtained 16 solutions for a variety of manipulators, leading to the hypothesis that this was the maximum number of real solutions. The following year Primrose [41] proved the 16 solution hypothesis to be correct using projective geometry. Papers by Lee and Liang in 1987 [42] and 1988 [43] extended work by Duffy to obtain a polynomial of degree 16 in the tangent of the half-angle of one of the joint variables.

In 1990 a paper by Raghavan and Roth [44] introduced an algorithm for a complete solution to the inverse kinematics problem for 6R-manipulators. Elimination methods are used on a set of nonlinear equations to obtain a polynomial of degree 16 which can be solved and then each solution back-substituted to obtain the 16 sets of joint angles. This algorithm is the basis for the work of many other researchers. One example is Manocha and Canny [45] in 1992 where the efficiency of the Raghavan-Roth algorithm is improved using symbolic preprocessing and Eigenvalues. Another Eigenvalue technique was developed in 1993 by Kohli and Osvatic [46] who use the Eigenvalues of a $16 \times 16$ matrix that represent the equations that are linear in one variable; a similar algorithm was introduced in the same 
year by Ghazvini [47] in which he used the fact that the equations of Raghavan and Roth are linear in the tangent of the half angle of the third joint variable to set up a generalized Eigenproblem.

The algorithms based on the method of Raghavan and Roth are the most well known though many other authors have provided their input on the inverse kinematics problem. In 2006 a new algorithm for the inverse kinematics of $6 \mathrm{R}$-manipulators that utilizes kinematic mapping and the constraint varieties of serial chains was presented in [48] and [2]. This new algorithm is the basis for the novel inverse kinematics work presented in this thesis. The kinematic mapping technique for the inverse kinematics is discussed in the following subsection.

\subsubsection{Inverse Kinematics of a 6R-manipulator Utilizing Kinematic Map- ping}

The method of solving the inverse kinematics problem for $6 \mathrm{R}$-manipulators using kinematic mapping was first introduced by Husty, Pfurner and Schröcker [48] and was further generalized by Pfurner [2]. This section provides an overview of these techniques focusing on those that are relevant to the work presented in this thesis.

Consider a prescribed EE target pose, $\Sigma_{\text {target }}$, for a known $6 \mathrm{R}$-manipulator. It is desired to obtain all sets of joint parameters required to make the EE frame, $\Sigma_{E E}$, coincident with $\Sigma_{\text {target }}$. This is illustrated for a general 6R-manipulator in Figure 3.4. The manipulator is theoretically "split" into two 3R-chains which will be called the left and right chains. The split is made where the third link meets the fourth joint. The left chain maintains the original base frame $\Sigma_{0}$, called $\Sigma_{0 L}$ when referring to the left chain, and the new EE frame of the left chain, $\Sigma_{L}$, is affixed at the break in the original chain. The left chain contains joints 1,2 and 3 from the original 6 R-chain. The new "base" of the right chain, $\Sigma_{0 R}$, is coincident with $\Sigma_{E E}$ in the target pose and the EE of the right chain, $\Sigma_{R}$ is coincident with $\Sigma_{L}$ before the chain is broken. That is, when $\Sigma_{L}$ and $\Sigma_{R}$ are coincident, the original $6 \mathrm{R}$-chain is obtained. The right chain contains joints 4,5 and 6 from the orginal $6 \mathrm{R}$-chain. From $\Sigma_{0 R}$ to $\Sigma_{R}$ the order of the joints is 6,5 then 4 . Figure 3.5 illustrates the split into 


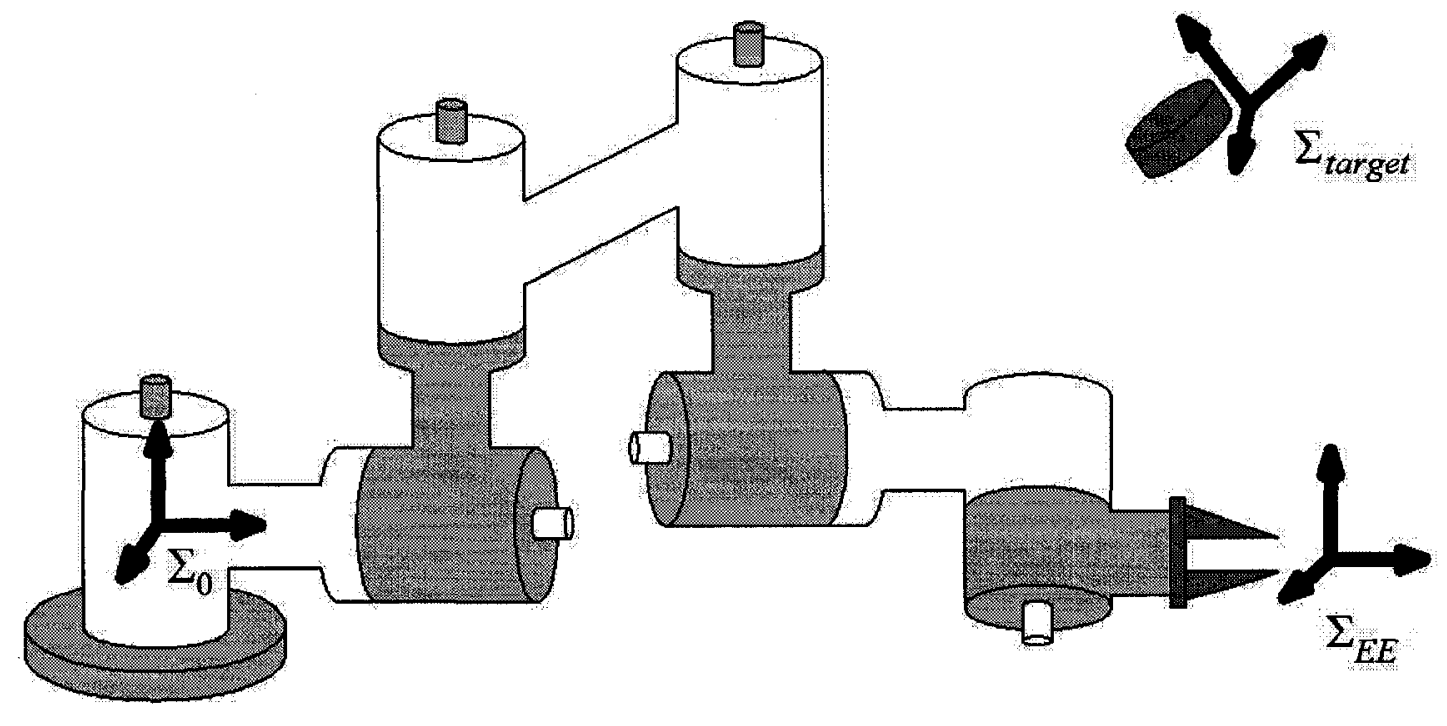

Figure 3.4: Illustration of inverse kinematics problem for a general 6R-manipulator.

the two 3R-chains and placement of reference frames for the left and right chains.
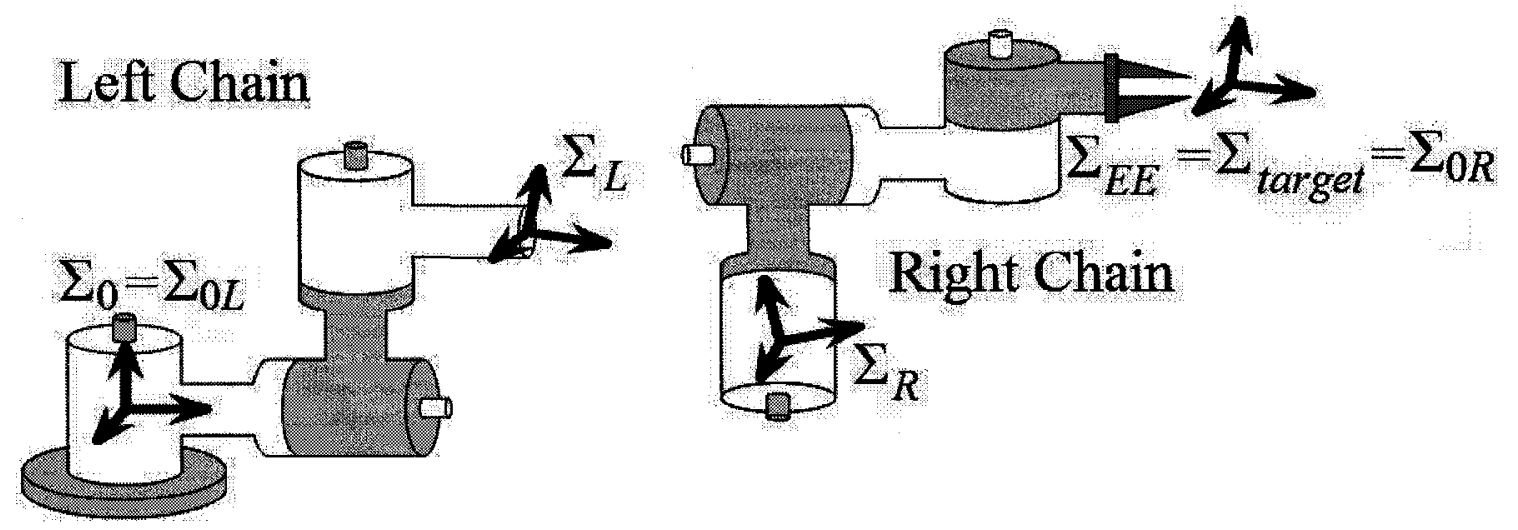

Figure 3.5: Illustration of left and right 3R-chains with reference frames.

The direct kinematic equations for the left and right chains are found using Equation (2.18). The constraint variety is then determined for each chain, providing the set of all points in $P^{7}$ that represent every displacement from the base frame to the EE frame achievable by the 3R-manipulator. If the target EE pose is within the workspace of the 6R-manipulator then the constraint varieties of the left and right chains will intersect in at least one real point in $P^{7}$. The set of all intersection points provides all the real sets of joint 
angles that keep $\Sigma_{L}$ and $\Sigma_{R}$ coincident and the 6 R-chain unbroken.

Theoretically this technique should apply to all six-jointed manipulators but requires the determination of the constraint varieties for each of the left and right chains, which varies for different combinations of joint types. Pfurner in [2] provides the derivation of the constraint varieties for 3R-chains and uses them to describe an algorithm to obtain the inverse kinematic solution to general 6R-manipulators.

Pfurner shows that the constraint variety of the 3R-chain is a well known geometric entity called a Segre manifold. This observation allows for simplification of the inverse kinematics algorithm for $6 \mathrm{R}$-manipulators, but has no application to this thesis and will not be discussed further, except to say that the intersection of the Segre manifolds for the left and right chains (with each Segre manifold consisting of four hyperplanes) with $S_{6}^{2}$ results in 16 intersection points. Some of these intersection points may be real, representing physically obtainable solutions to the inverse kinematics problem (ignoring joint limits and self-collisions); and some may be complex conjugate, which have no physical meaning but still mathematically result in $\Sigma_{L}$ and $\Sigma_{R}$ being coincident. The maximum 16 real intersection points represents the maximum 16 solutions to the inverse kinematics problem. More information about Segre manifolds and the intersection of these special constraint varieties to solve the inverse kinematics problem is found in [2].

It is useful to look at the procedure Pfurner initially uses to obtain the 3R-chain constraint variety, before using the knowledge that it is represented by a Segre manifold, so that the methods may be applied to other joint types. The constraint variety for a 3R-chain is built by first looking at a canonical 2R-chain. The term canonical means that the reference frames are placed such that the chain can be represented in the simplest way without loss in generality. In this case canonical configuration is such that the base and first reference frames are coincident when the first joint variable is zero, the second reference frame has its origin where link 1 intersects the second joint axis and the third reference frame origin is where link 2 intersects the third joint axis. The direct kinematics equations are found and kinematic mapping is used to find the Study parameters in terms of the two unknown joint variables. Elimination of the joint variables by substitution gives four hyperplane equations 
in $P^{7}$, that is four equations that are linear in the Study parameters $x_{i}, y_{i}, i=0, \ldots, 3$. The intersection of these four hyperplanes with the Study quadric is the constraint variety of the $2 \mathrm{R}$-chain. The results for the $2 \mathrm{R}$-constraint variety can be transformed to the canonical 3R-chain using the appropriate $\mathbf{T}_{b}$ and $\mathbf{T}_{m}$ matrices of Equations (2.20) and (2.21). New joints are added in the base or moving frame as required. Adding a new joint to the end of the chain is described here, but the interested reader is referred to [2] for other possibilities. Since the 2R-chain is canonical, the second link has not yet been accounted for, as well as the newly added joint and third link, described by matrices $\mathbf{G}_{2}, \mathbf{M}_{3}$ and $\mathbf{G}_{3}$ respectively. $\mathbf{M}_{3}$ contains the joint variable $u_{3}$, using the tangent of the half-angle substitution, the other matrices are constant. The hyperplane equations of the 2R-chain are put into vector form, such that the hyperplane coordinates (the coefficients of the ordered terms in the hyperplane equations) are given in vector $\mathbf{u}$. These hyperplane coordinates are then transformed by the equation $\widetilde{\mathbf{u}}=\left(\left(\mathbf{T}_{b}\left(\mathbf{G}_{3}\right) \mathbf{T}_{b}\left(\mathbf{M}_{3}\right) \mathbf{T}_{b}\left(\mathbf{G}_{2}\right)\right)^{T}\right)^{-1} \mathbf{u}$, where $\widetilde{\mathbf{u}}$ is a vector containing the transformed hyperplane coordinates in terms of the joint variables. The equation to perform the transformation is obtained by concatenating multiple transformations. The resulting equations remain linear in the Study parameters and the intersection of these four new one-parameter hyperplanes with $S_{6}^{2}$ is the constraint variety of the 3R-chain.

The solution to the inverse kinematics problem comes from intersecting the constraint varieties of the left and right 3R-chains. For each chain there are four one-parameter hyperplane equations intersecting $S_{6}^{2}$ meaning that in total there are nine equations: Eight one-parameter hyperplane equations and $S_{6}^{2}$. The unknowns are the two joint parameters, one from each of the left and right chains, and the eight homogeneous Study parameters. Because they are homogeneous coordinates, one of the Study parameters may, without loss in generality, be set to 1 , leaving nine unknowns in all. The real solutions to this set of nine equations and nine unknowns, after back substitution to find the four remaining joint parameters, provides the solution to the inverse kinematics of the $6 \mathrm{R}$-manipulator. The sum of real and complex conjugate solutions is always 16 .

The work presented in this chapter is the basis for the original contributions of this thesis and is used directly or as a guideline for the work presented in the following chapters. 


\section{Chapter 4}

\section{Introduction to A-Pairs and A-Chains}

It is useful at this time to reiterate the purpose of this thesis: to examine the effects on the direct and inverse kinematics of a $6 \mathrm{R}$-serial manipulator whose revolute joints are replaced by a novel type of joint comprising a special configuration of GDP that is subject to a specific $1 D O F$ self-motion. This chapter begins the presentation of the original work of this thesis by clarifying the configuration and nomenclature for the new joint, then describes a kinematic chain built using these special joints, examines the direct kinematics of the kinematic chain and finally derives the basic constraint varieties for some simple kinematic chains. The work of this chapter is used in the Chapter 5 as part of the algorithm for the inverse kinematics of special kinematic chains utilizing the new kinematic pairs as joints.

\subsection{The A-Pair Using a GDP as a Kinematic Pair}

The new type of kinematic pair is termed an A-pair. The configuration of GDP comprising the A-pairs replacing the R-pairs of a kinematic chain for the purposes of this thesis is of the midline-to-vertex type with congruent, equilateral base and platform triangles and all six legs being the same fixed length such that the path of any point on the platform undergoing a self-motion is the same as that described in Section 3.1.2. The fixed-length legs of the GDPs are passive such that the only relative motion between each fixed base and moving platform is due to the self-motion phenomenon. To simplify the computations the added constraint that the leg lengths are equal to the height of the congruent fixed base 
and moving platform triangles is used. This is so the entire range of self motions can be obtained without disassembling and reassembling the joint. For the purposes of this thesis self-collisions have been ignored, that is it is assumed that the individual solid bodies that make up the fixed base, moving platform and legs of the A-pair can pass through each other and occupy the same points in space at the same instant. In the described configuration it has been shown in Section 3.1.2 that self-motions result in a coupling of rotation and translation of the platform such that the displacement of the platform along the joint axis mutually perpendicular to the fixed base and moving platform, $d$, is related to the rotation angle about the joint axis, $\theta$, by the function

$$
d=\rho \sin \left(\frac{\theta}{2}\right)
$$

where $\rho$ is set equal to unity for convenience. When replacing an $\mathrm{R}$-joint in a chain with the special configuration of GDP the axis of the joint is unchanged. The base of the GDP connects to one link, $i-1$, and the platform connects to the other link, $i$, such that if link $i-1$ is fixed, link $i$ will rotate and translate relative to $i-1$. The base or ground link is called link 0 (corresponding to $i=0$ ). The joint axis is the line mutually perpendicular to the fixed base and moving platform and that passes through the geometric centres of the fixed base and moving platform triangles.

The coupling of rotation and translation leads to a kinematic pair which may be referred to as an algebraic screw pair. In a traditional screw pair (or helical pair, H-pair) the relationship between the rotation and translation is linear, while in the case of this special configuration of GDP being used as a kinematic pair this relationship is an algebraic equation (after utilizing the tangent of the half-angle substitution to eliminate trigonometric functions). With this nomenclature the GDP joints are a form of algebraic screw pair, or A-pair. Replacing the R-pair joints of the 6R-manipulator with A-pair joints makes it a 6A-manipulator. There exist many possible A-pair configurations but for the purposes of this thesis the term A-pair refers to the kinematic pair and joint in a kinematic chain constructed using the special configuration of GDP described previously. An example of 
a possible application for an A-pair jointed manipulator is shown in Figure 4.1 where the robotic manipulator of the Space Shuttle or International Space Station has had the R-pair joints replaced with A-pairs.
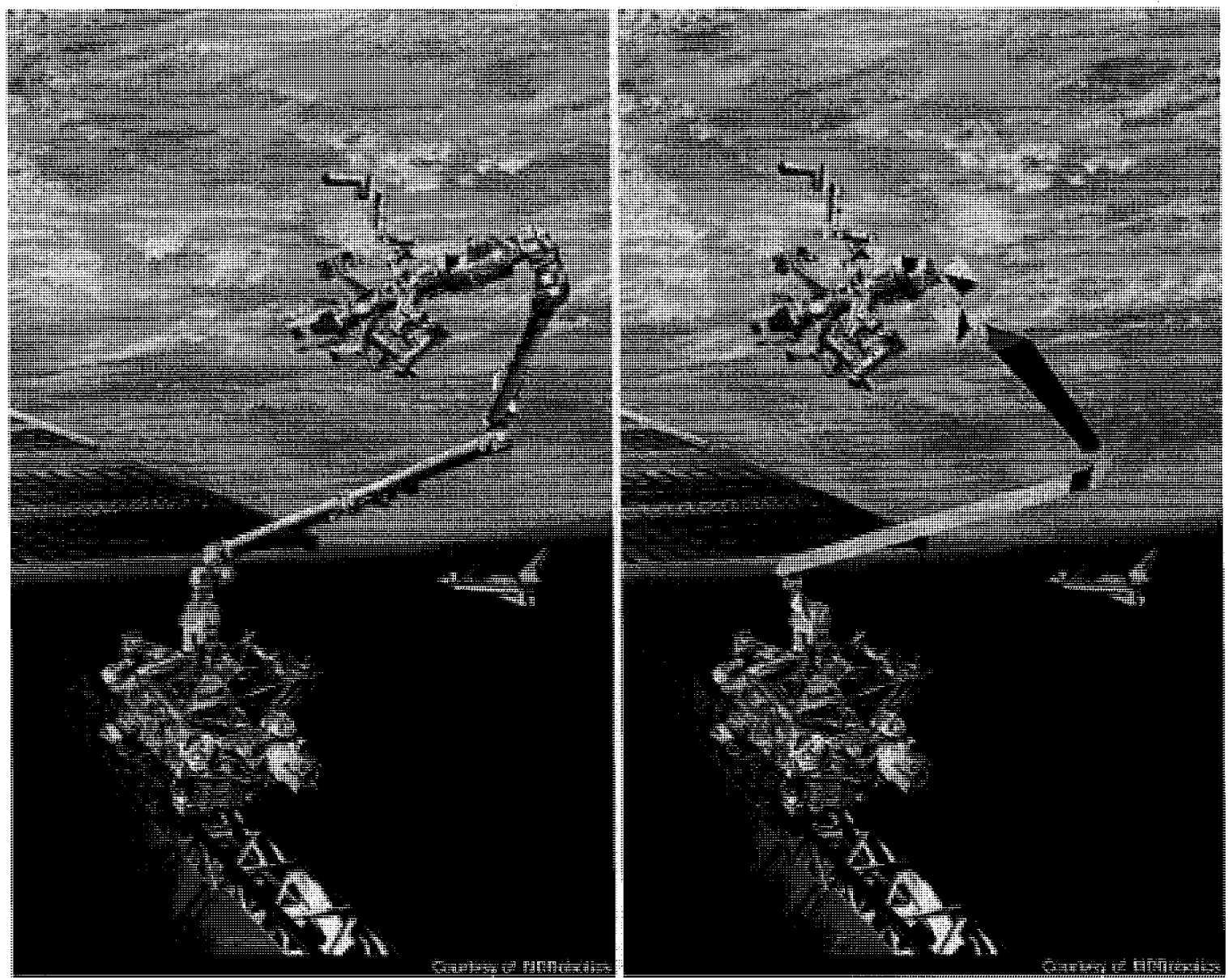

Figure 4.1: Example application of A-pair jointed manipulator where the R-pair joints of the International Space Station robot arm (left) are replaced (right) with A-pair joints.

The generally lower stiffness-to-weight ratio of serial manipulators results from both the cantilever links as well as clearances and structural stiffness of the R-pair joints themselves. The joint clearance and stiffness issue affects both the accuracy of the intended rotation about the axis as well as introducing undesired motion brought on by moments applied out of the plane of rotation and forces in all directions. Stiffening the joints requires stiffening the structure of the joint and bearings, which generally requires adding weight. It is hypothesized, with no evidence, that the generally higher stiffness-to-weight ratio of parallel 
manipulators, as discussed in Section 2.3, means the A-pair joint improves the resistance of the joint to undesired motion due to the forces and moments not about the joint axis. A traditional R-pair joint connects two links by one path along the axis of the joint. The A-pair joint on the other hand has six connections between the two links. Assuming the same stiffness for each connection on both joint types the A-pair has six connections in parallel (stiffness is added for links in parallel,) so six times the stiffness. The EE of the $6 \mathrm{~A}$-manipulator is still at the end of a cantilever arm, but based on the hypothesis that the A-pair joints of the kinematic chain are inherently stiffer, the stiffness-to-weight ratio of the entire manipulator should be increased. Space applications, as suggested by Figure 4.1 , is one area that would benefit from the higher stiffness-to-weight ratio because lighter take-off weights mean lower costs, while functionality is enhanced. The addition of the $1 \mathrm{DOF}$ coupled rotation and translation requires an examination of the manipulator direct and inverse kinematics.

When a single R-pair in a serial chain is actuated the pose of the EE frame changes relative to the base frame such that there is a rotation about the joint axis. For an A-pair the motion is different, the axis and magnitude of a rotation is unchanged, but there is now a translation parallel to the joint axis that is a sinusoidal function of the joint angle. The addition of coupled rotation and translation necessitates an examination of the direct kinematics of the new manipulator, similarly the inverse kinematics algorithm must be re-evaluated. The inverse kinematics algorithm introduced by Pfurner and presented in Section 3.2 applies only to $6 \mathrm{R}$-chains and must be modified to be applicable to other joint types. A major contribution of this thesis is the exploration of the inverse kinematics of an A-pair jointed manipulator and presents an algorithm for the inverse kinematics that parallels that presented by Pfurner.

\subsection{Setup of A-Chains}

In order to fully define the A-pair jointed chain, which is called an A-chain, a complete description of the joint constructed with an A-pair and the links in the A-chain is required. 
With a fully defined chain it is possible to examine the kinematics of a manipulator constructed using A-pairs. This section defines how the A-pair is used as a joint in a kinematic chain and shows how the A-chain is assembled. DH-parameters are assigned, accounting for the new joint type.

\subsubsection{A-Chain Configuration}

To fully describe an A-chain it must be clear how the kinematic pair is incorporated into a kinematic chain. The separation of the base and platform of an A-pair undergoing selfmotion is given by $\sin \left(\frac{\theta_{i}}{2}\right)$, as is derived in Section 3.1.2, where $\theta_{i}$ is the angle of rotation of the moving platform of the A-pair relative to the fixed base for joint $i$. The zero position $\left(\theta_{i}=0\right)$ of the A-pair is the theoretical position where the fixed base and moving platform coincide. If the leg length were not equal to the height of the fixed base and moving platform triangles then in the zero position the legs we be either too long or not long enough to connect the anchor points of the fixed base and moving platform. This position is only attainable if self-collisions are ignored, but is useful as a conceptual home position.

To understand the construction of the A-chain it is useful to look at two links, link $i-1$ and link $i$. The two links are connected by joint $i$ such that link $i-1$ is connected to the fixed base of the A-pair and link $i$ is connected to the moving platform. This is repeated for all links in the chain. As the joint angles change, links $i-1$ and $i$ rotate relative to each other about joint axis $i$ and the offset between the two links varies due to the coupling of translation and rotation in the joint.

\subsubsection{The DH-Parameters of an A-Chain}

In order to examine the kinematics of the new serial manipulator it is necessary to have a unambiguous definition of the A-chain, this can be done using the DH-parameters introduced in Section 2.5.1. Applying DH-parameters to A-chains requires some discussion since changing the joint variable alters more than one DH-parameter.

For an $n \mathrm{~A}$-manipulator with $i=0, \ldots, n$, the joint axis is identified for each A-pair, as are the common normals or between adjacent joint axes or their intersection point. The 
reference frames are then established in the same manner as in Section 2.5.1. The definitions of the link length, $a_{i}$, link twist, $\alpha_{i}$ and joint angle, $\theta_{i}$, remain unchanged from the R-chain. In the A-chain the joint offset, $d_{i}$, is now variable, coupled with the already variable $\theta_{i}$. The $d_{i}$ term is broken into two parts, one fixed and one variable. The variable part is known for the A-pair and is equal to $\sin \left(\frac{\theta_{i}}{2}\right)$. The fixed part depends on the architecture of the manipulator and has the same definition as in Section 2.5 .1 only when joint $i$ is in the zero position. The fixed part of the joint offset is still referred to as $d_{i}$ while the variable part remains as a function of the joint angle as to avoid the introduction of more variables. Figure 4.2 shows how the DH-parameters are applied to a link connecting two A-pair joints.

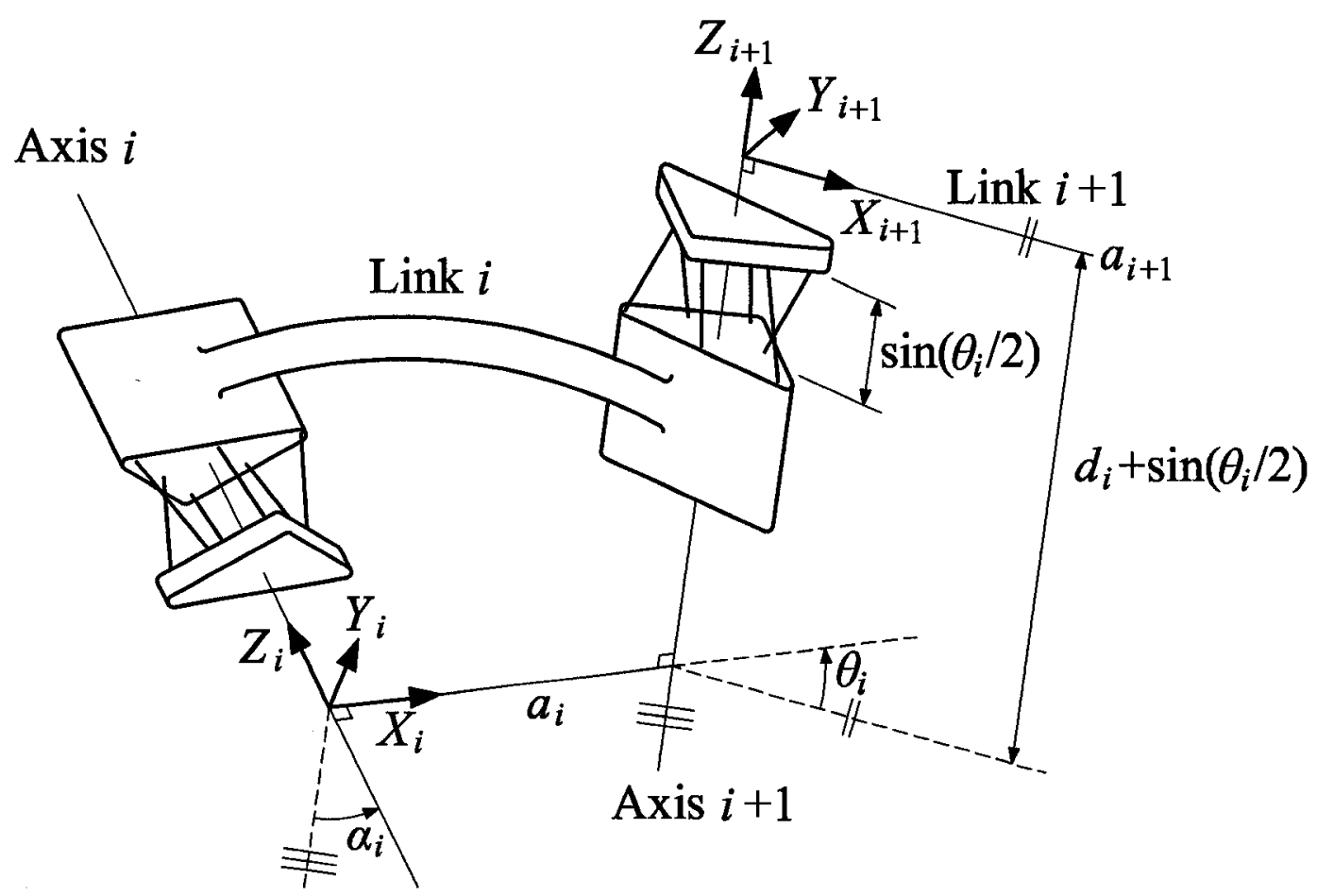

Figure 4.2: The DH-parameters applied to a link in an A-chain.

Utilizing these $\mathrm{DH}$-parameters it is now possible to obtain the direct kinematic equations for a general A-chain with any number of A-pair joints. 


\subsection{Direct Kinematics of A-Chains}

The direct kinematic equations of an $n \mathrm{~A}$-chain are obtained using Equation (2.18), with some changes to the $\mathbf{M}_{i}$ matrices. The $\mathbf{G}_{i}$ matrix remains unchanged with the new definition of $d_{i}$, while $\mathbf{M}_{i}$ is altered such that

$$
\mathbf{M}_{\mathbf{i}}=\left[\begin{array}{cccc}
1 & 0 & 0 & 0 \\
0 & \cos \left(2 \theta_{i}\right) & -\sin \left(2 \theta_{i}\right) & 0 \\
0 & \sin \left(2 \theta_{i}\right) & \cos \left(2 \theta_{i}\right) & 0 \\
\sin \left(\theta_{i}\right) & 0 & 0 & 1
\end{array}\right]
$$

This new $\mathbf{M}_{i}$ matrix will be used throughout the remainder of this thesis and varies from the original presented in Section 2.5.2 in that the term $\sin \left(\frac{\theta_{i}}{2}\right)$ is the $(4,1)$ element of $\mathbf{M}_{i}$ and then $\theta_{i}$ was replaced by $2 \theta_{i}$ throught the entire matrix to remove fractions. This change to $\mathbf{M}_{i}$ has the same effect as altering the definition of $d_{i}$ such that $d_{i}=d_{i}+\sin \left(\frac{\theta_{i}}{2}\right)$ and maintaining the original $\mathbf{M}_{i}$ and $\mathbf{G}_{i}$ matrices, however the new matrices are used such that the $\mathbf{G}_{i}$ matrices remain constant, while all the terms affected by changing the joint variables are contained in $\mathbf{M}_{i}$.

It quickly becomes impractical to show the substitution of the new $\mathbf{M}_{i}$ and $\mathbf{G}_{i}$ matrices in Equation (2.18) for a completely general manipulator, but an examination of the structure of the matrices quickly reveals the effect of introducing the coupled rotation and translation. Recalling that in the general transformation matrix $\mathbf{T}$ of Equation (2.4) the first column represents the position vector of the origin of the frame $\Sigma^{\prime}$ relative to $\Sigma$ and the lower right $3 \times 3$ sub matrix represents the orientation of $\Sigma^{\prime}$ relative to $\Sigma$, the effect of the new joint can be seen. Since the $\sin \left(\theta_{i}\right)$ term representing the translation part of the coupled joint motion appears only in the first column of $\mathbf{M}_{i}$ and the first row of both $\mathbf{M}_{i}$ and $\mathbf{G}_{i}$ is always $\left[\begin{array}{llll}1 & 0 & 0 & 0\end{array}\right]$. It can be seen that any term in the first column will, after multiplication, remain only in the first column of the product matrix. This means that if the R-pairs of an $n \mathrm{R}$-manipulator are replaced with $\mathrm{A}$-pairs and the home positions of all joints are identical the orientation of the EE reference frame for any set of joint variables would be unchanged 
while only the position in space would be different.

A short numeric example illustrates the difference in EE pose for a 3R-manipulator and a 3A-manipulator. Consider two manipulators with the same unit-less DH-parameters presented in Table 4.1. One serial chain uses A-pairs as joints and the other uses R-pairs. For the 3R-chain the values of Table 4.1 are substituted into Equations (2.16) and (2.17)

Table 4.1: DH-parameters for 3A-manipulator direct kinematics numeric comparison.

\begin{tabular}{|c||c|c|c|c|}
\hline link $i$ & $a_{i}$ & $\alpha_{i}(\mathrm{deg})$ & $d_{i}$ & $\theta_{i}(\mathrm{deg})$ \\
\hline \hline 1 & 3 & 20 & 0 & 25 \\
\hline 2 & 1 & -45 & $\frac{2}{3}$ & 95 \\
\hline 3 & $\frac{1}{2}$ & 32 & 3 & -16 \\
\hline
\end{tabular}

and the matrix representing the EE pose is found to be, using Equation (2.18),

$$
\mathbf{T}_{\mathbf{3 R}}=\left[\begin{array}{cccc}
1.0 & 0.0 & 0.0 & 0.0 \\
0.676057383 & -0.2588263850 & -0.9659238481 & 0.0001491768 \\
0.572791274 & 0.8162455167 & -0.2188015513 & -0.5346673147 \\
3.155575269 & 0.5164805501 & -0.1382642434 & 0.8450626251
\end{array}\right]
$$

For the 3A-chain the values of Table 4.1 are substituted into Equations (2.16) and (4.1) and the matrix representing the EE pose is found to be, using Equation (2.18),

$$
\mathbf{T}_{\mathbf{3 A}}=\left[\begin{array}{cccc}
1.0 & 0.0 & 0.0 & 0.0 \\
0.853846137 & -0.2588263850 & -0.9659238481 & 0.0001491768 \\
0.423494442 & 0.8162455167 & -0.2188015513 & -0.5346673147 \\
3.975287088 & 0.5164805501 & -0.1382642434 & 0.8450626251
\end{array}\right]
$$

As can be seen the $3 \times 3$ lower right matrices, representing the EE orientation, of $\mathbf{T}_{\mathbf{3 R}}$ and $\mathbf{T}_{\mathbf{3 A}}$ are identical. The first columns of the two matrices, representing the position of the origin of the $\mathrm{EE}$ reference frame in space, is different. 
In a chain with mixed joint types, such as a combination of R-, P- and A-pairs, the appropriate $\mathbf{M}$ and $\mathbf{G}$ matrices are used for the different joints in the chain and substituted into Equation (2.18).

Obtaining the direct kinematics equations of an A-chain is conceptually no more difficult than for an R-jointed chain. For and $n \mathrm{~A}$-chain the orientation of the EE reference frame remains unchanged relative to the equivalent $n \mathrm{R}$-chain, the difference in EE position between the A- and R-chains for common joint variables means the inverse kinematics algorithm must be re-evaluated.

\subsection{Obtaining the Constraint Varieties of Canonical A- Chains}

There is a difference in pose of the EE for common sets of joint angles between $n \mathrm{~A}$ - and $n$ R-manipulators that have the same $\mathrm{DH}$-parameters and zero joint angle positions. This is not a surprising result since the motion of A-pairs differs from that of R-pairs in that there is a translation component of motion coupled with the rotation of the A-pair. It is important to understand the differences when solving the inverse kinematics.

In Section 3.2 several methods are introduced that aim to solve the inverse kinematics problem for serial manipulators. Several of the methods are only applicable to a specific manipulator configuration. For example Pieper's method, which is commonly used on wrist-partitioned industrial robots, requires three consecutive intersecting joint axes. For a manipulator with A-pair joints it is not possible to enforce this restriction because the joint axes move as the the various joints rotate and translate, so axes that intersect at one instant may not intersect at another.

Pfurner's algorithm, presented in Section 3.2.2, uses the constraint varieties of R-chains to solve the inverse kinematics problem for $6 \mathrm{R}$-manipulators. This method is not directly applicable to $6 \mathrm{~A}$-manipulators but a hypothesis of this thesis is that the same basic theory may be used to develop an algorithm that gives all sets of joint angles for a desired pose of a $6 \mathrm{~A}$-manipulator. This requires the derivation of the constraint varieties for $1 \mathrm{~A}-, 2 \mathrm{~A}$ - and 
ultimately 3A-chains. This chapter presents the derivation of canonical single A-pair and 2A-chain constraint varieties. The term canonical is used to describe chains that possess reference frames in the simplest position to describe the chain without loss in generality. In the next chapter these varieties will be adjusted to non-canonical form and then used to solve the inverse kinematics of a 4A-manipulator and, after a third joint parameter is added, set up the equations to solve the inverse kinematics of a $6 \mathrm{~A}$-manipulator. The constraint varieties of $3 \mathrm{~A}$-chains are used in a different form than the single $\mathrm{A}$-pair and $2 \mathrm{~A}$-chain and their derivation is presented in Chapter 5 .

\subsubsection{Constraint Variety of a Single A-pair}

A canonical A-pair is simply a GDP with one frame affixed to the base, $\Sigma_{i-1}$, and another to the platform, $\Sigma_{i}^{\prime}$. The $Z$ axis of each reference frame is along the joint axis. In the theoretical zero position these two frames are coincident. As the platform moves under self-motion $\Sigma_{i}^{\prime}$ moves relative to $\Sigma_{i-1}$ such that the motion is represented by the matrix $\mathbf{M}_{i}$ defined by Equation (4.1). The canonical A-pair with reference frames $\Sigma_{i-1}$ and $\Sigma_{i}^{\prime}$ is shown in Figure 4.3.

Using trigonometric identities to eliminate the terms containing $2 \theta_{i}, \mathbf{M}_{i}$ can be written as

$$
\mathbf{M}_{\mathbf{i}}=\left[\begin{array}{cccc}
1 & 0 & 0 & 0 \\
0 & \cos ^{2}\left(\theta_{i}\right)-\sin ^{2}\left(\theta_{i}\right) & -2 \sin \left(\theta_{i}\right) \cos \left(\theta_{i}\right) & 0 \\
0 & 2 \sin \left(\theta_{i}\right) \cos \left(\theta_{i}\right) & \cos ^{2}\left(\theta_{i}\right)-\sin ^{2}\left(\theta_{i}\right) & 0 \\
\sin \left(\theta_{i}\right) & 0 & 0 & 1
\end{array}\right],
$$

and performing the tangent of the half-angle substitution gives

$$
\mathbf{M}_{\mathbf{i}}=\left[\begin{array}{cccc}
1 & 0 & 0 & 0 \\
0 & \frac{\left(1-u_{i}^{2}\right)^{2}}{\left(1+u_{i}^{2}\right)^{2}}-\frac{4 u_{i}^{2}}{\left(1+u_{i}^{2}\right)^{2}} & -\frac{4 u\left(1-u_{i}^{2}\right)}{\left(1+u_{i}^{2}\right)^{2}} & 0 \\
0 & \frac{4 u\left(1-u_{i}^{2}\right)}{\left(1+u_{i}^{2}\right)^{2}} & \frac{\left(1-u_{i}^{2}\right)^{2}}{\left(1+u_{i}^{2}\right)^{2}}-\frac{4 u_{i}^{2}}{\left(1+u_{i}^{2}\right)^{2}} & 0 \\
\frac{2 u}{1+u_{i}^{2}} & 0 & 0 & 1
\end{array}\right]
$$




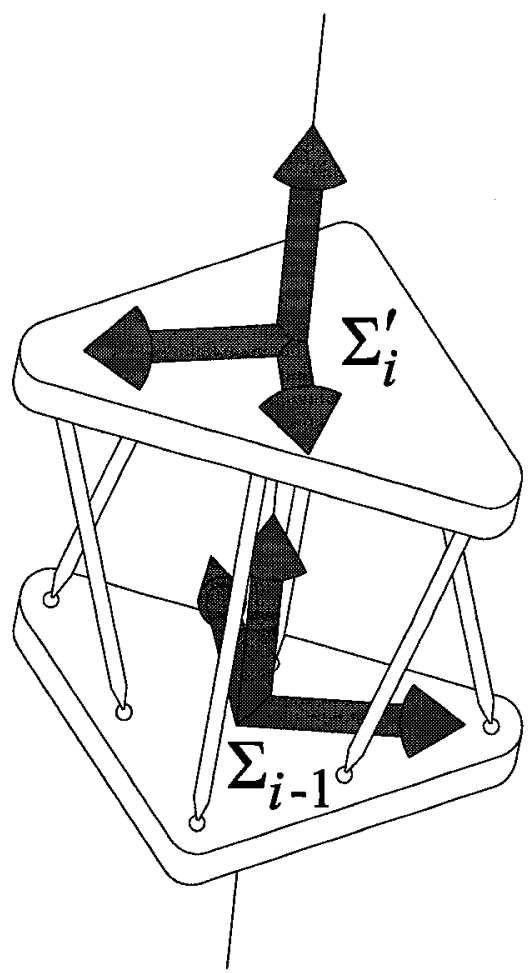

Figure 4.3: Canonical 1A-pair with reference frames $\Sigma_{i-1}$ and $\Sigma_{i}^{\prime}$.

where $u_{i}=\tan \left(\frac{\theta}{2}\right)$. The Study parameters of this matrix are now obtained using Equations (2.14) and (2.15) and simplify to

$$
\left(\begin{array}{l}
x_{0} \\
x_{1} \\
x_{2} \\
x_{3} \\
y_{0} \\
y_{1} \\
y_{2} \\
y_{3}
\end{array}\right)=\left(\begin{array}{c}
1-u_{i}^{4} \\
0 \\
0 \\
2 u_{i}\left(1+u_{i}^{2}\right) \\
2 u_{i}^{2} \\
0 \\
0 \\
u_{i}\left(u_{i}^{2}-u_{i}\right)
\end{array}\right) \text {. }
$$

In order to obtain the constraint variety in $P^{7}$ it is necessary to eliminate the joint variable $u_{i}$ from Equation (4.3). Because the Study space a seven dimensional projective 
space and there is one joint variable, $u_{i}$, six independent equations are required to define the constraint manifold in this space. Examination of the study parameters and utilization of the resultant method of elimination, presented in Section 2.9, the following set of six equations is obtained:

$$
\begin{aligned}
& \text { 1: } x_{0} y_{0}+x_{1} y_{1}+x_{2} y_{2}+x_{3} y_{3}=0 \text {, } \\
& \text { 2: } \quad x_{1}=0 \text {, } \\
& 3: \quad x_{2}=0 \text {, } \\
& \text { 4: } y_{1}=0 \text {, } \\
& \text { 5: } y_{2}=0 \text {, } \\
& \text { 6: } \quad x_{3}^{2}-4 y_{0}^{2}-4 y_{3}^{2}=0 \text {. }
\end{aligned}
$$

The intersection of these equations represent the constraint variety of a single canonical Apair. It is believed that this is the simplest set of equations which represent the constraint variety, though this has yet to be proven. Examination of the set of equations in Equation (4.4) shows that the constraint variety is the intersection of four hyperplanes and one quadric with $S_{6}^{2}$.

\subsubsection{Constraint Variety of Canonical 2A-Chains}

The constraint variety for a single canonical A-pair can now be used to obtain the constraint variety for the canonical 2A-chain. This is done by applying the methods of Section 2.6.1 to first transform $\Sigma_{i}^{\prime}$ from the platform of the first GDP to the end of link $i$ and then to introduce a second joint variable. A canonical $2 \mathrm{~A}$-chain with attached reference frames is shown in Figure 4.4. $\Sigma_{i}$ is a reference frame affixed to the fixed base of the new A-pair with axis $Z_{i}$ along the axis of the second A-pair. Reference frame $\Sigma_{i+1}^{\prime}$ is affixed to the moving platform of the second A-pair with the $Z_{i+1}^{\prime}$ axis along the joint axis of the second A-pair. When the joint variable for the second A-pair is zero $\Sigma_{i}$ and $\Sigma_{i+1}^{\prime}$ are coincident.

The constant link parameters are accounted for first. This requires a transformation of $\Sigma_{i}^{\prime}$ from the moving platform of the first A-pair to $\Sigma_{i}$ on the fixed base of the second A-pair. 


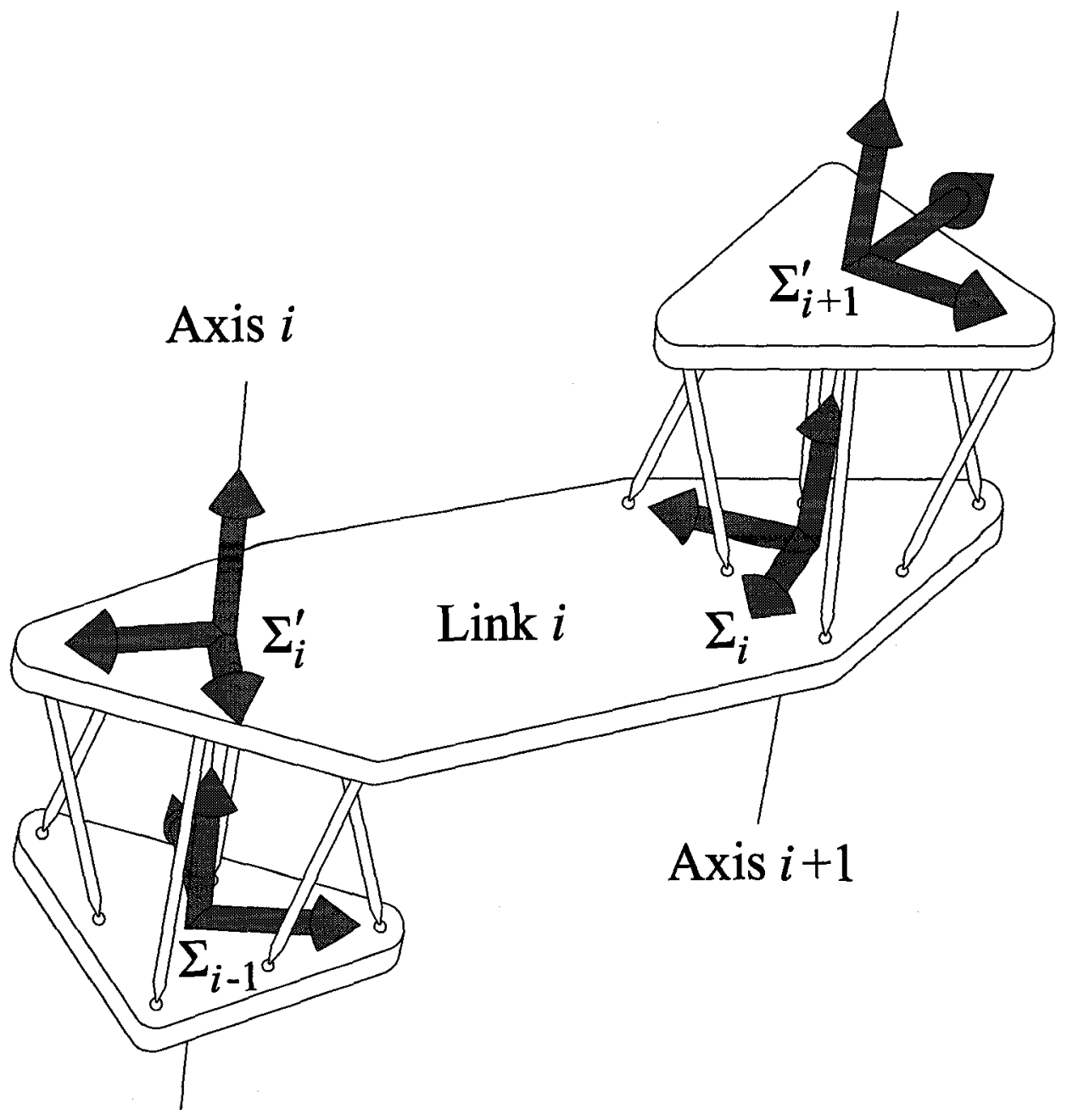

Figure 4.4: Canonical 2A-chain with reference frames $\Sigma_{i-1}, \Sigma_{i}^{\prime}, \Sigma_{i}$ and $\Sigma_{i+1}^{\prime}$.

This is done using the matrix $\mathbf{G}_{i}$ which, after the tangent of the half-angle substitution, becomes

$$
\mathbf{G}_{i}=\left[\begin{array}{cccc}
1 & 0 & 0 & 0 \\
a_{i} & 1 & 0 & 0 \\
0 & 0 & \frac{1-a l_{i}^{2}}{1+a l_{i}^{2}} & -\frac{2 a l_{i}^{2}}{1+a l_{i}^{2}} \\
d_{i} & 0 & \frac{2 a l_{i}^{2}}{1+a l_{i}^{2}} & \frac{1-a l_{i}^{2}}{1+a l_{i}^{2}}
\end{array}\right]
$$

where $a l_{i}=\tan \left(\frac{\alpha_{i}}{2}\right)$.

The Study parameters of $\mathbf{G}_{i}$ are obtained and substituted into the $8 \times 8$ transformation matrix for the moving frame given in Equation (2.21) yielding $\mathbf{T}_{m}\left(\mathbf{G}_{i}\right)$. The transformed 
equations of the canonical single A-joint in Equation (4.4) are obtained by substituting $\mathbf{x}^{\prime}$ for $\mathbf{x}$ where

$$
\mathbf{x}^{\prime}=\mathbf{T}_{m}\left(\mathbf{G}_{i}\right) \cdot \mathbf{x}
$$

and the vectors $\mathbf{x}$ and $\mathbf{x}^{\prime}$ are $\left[\begin{array}{llllllll}x_{0} & x_{1} & x_{2} & x_{3} & y_{0} & y_{1} & y_{2} & y_{3}\end{array}\right]$ and $\left[\begin{array}{llllllll}x_{0}^{\prime} & x_{1}^{\prime} & x_{2}^{\prime} & x_{3}^{\prime} & y_{0}^{\prime} & y_{1}^{\prime} & y_{2}^{\prime} & y_{3}^{\prime}\end{array}\right]$ respectively. The intersection of the resulting equations represents the constraint variety of the single A-pair that is no longer canonical because the reference frame of interest now is $\Sigma_{i}$ at the fixed base of the second A-pair, not $\Sigma_{i}^{\prime}$ as was used for the single A-pair (See Figure 4.4).

The effect of the second A-pair on the constraint manifold can be found as follows. The $8 \times 8$ transformation matrix in the moving frame, $\mathbf{T}_{m}\left(\mathbf{M}_{i+1}\right)$, is built by substituting the Study parameters of a single A-pair (Equation (4.3), with $u_{i+1}$ substituted for $u_{i}$ ) in Equation (2.21). Equation (4.6) is used with $\mathbf{M}_{i+1}$ replacing $\mathbf{G}_{i}$. The new set of equations represents the constraint variety for reference frame $\Sigma_{i+1}^{\prime}$ located on the moving platform of the newly added A-pair. The resulting set of equations contain the joint variable $u_{i+1}$. The equations are shown in Appendix A.

It is now desirable to obtain a set of equations independent of $u_{i+1}$ that represents the constraint variety of a canonical $2 \mathrm{~A}$-chain. In $P^{7}$ with two joint variables $\left(u_{i}\right.$ and $\left.u_{i+1}\right)$ five equations are required to fully represent the constraint variety. Elimination of $u_{i+1}$ using resultant methods produces equations that become excessively large and are not practical or efficient to use in software applications, hence a desire to use the simplest set of equations that spans the same ideal. Simpler equations are found by obtaining the Gröbner basis with lexographic term ordering, where $x_{0}<x_{1}<x_{2}<x_{3}<y_{0}<y_{1}<y_{2}<y_{3}$, which produces 
the set of equations:

$$
\begin{aligned}
1: & x_{0} y_{0}+x_{1} y_{1}+x_{2} y_{2}+x_{3} y_{3}=0, \\
2: & 2 a l_{1} y_{2} x_{1}+a_{1} x_{1}^{2}+2 a l_{1} y_{3} x_{2}+a_{1} x_{2}^{2}=0, \\
3: & a l_{1}^{2} x_{0}^{2}-x_{1}^{2}-x_{2}^{2}+a l_{1}^{2} x_{3}^{2}=0, \\
4: \quad 2 a l_{1}^{3} a_{1} y_{0}^{2}+2 a l_{1} a_{1} y_{1}^{2}+2 a l_{1} a_{1} y_{2}^{2}+2 a l_{1}^{3} a_{1} y_{3}^{2}+\left(a l_{1}^{4} a_{1}^{2}+2 a l_{1}^{2}+a_{1}^{2}\right) y_{1} x_{1} & =0, \\
+a l_{1}^{2} a_{1} x_{0} x_{1}+\left(a l_{1}^{4} a_{1}^{2}+2 a l_{1}^{2}+a_{1}^{2}\right) y_{2} x_{2} & \\
5: \quad 2 a l_{1} a_{1} y_{2} x_{1} x_{3}+2 a l_{1} a_{1} y_{1} x_{2} x_{3}-a l_{1} x_{0} x_{2} x_{3} & =0 .
\end{aligned}
$$

It has yet to be proven that this is the simplest set of equations describing the ideal. Note that as expected $S_{6}^{2}$ is unchanged by the transformations. The intersection of these equations represents the constraint variety of canonical $2 \mathrm{~A}$-chains. The intersection of the equations, as can be seen in Equation (4.7), consists of three quadric equations and one cubic equation intersecting with $S_{6}^{2}$.

The inverse kinematics of a $6 \mathrm{~A}$-manipulator requires the construction of the set of equations describing the constraint variety for a $3 \mathrm{~A}$-chain as a function of the third joint variable, $u_{i+2}$. Though the four equations required to describe the constraint variety independent of $u_{i+2}$ could be obtained, as will be shown in the next chapter it is not required for the inverse kinematics algorithm and therefore is not shown in this section. In the algorithm presented by Pfurner presented in Section 3.2 the constraint variety of a 3R-chain was shown to be a Segre manifold. The characteristics of this specific manifold were used to simplify the algorithm. No such simplification is obvious for the 3A-chain. The derivation of the 3A-chain constraint variety (in terms of $u_{i+2}$ ) appears in Section 5.2, in the set-up of the solution for the inverse kinematics of a $6 \mathrm{~A}$-manipulator. 


\section{Chapter 5}

\section{Algorithm for the Inverse Kinematics of A-Chains}

The complete solution to the inverse kinematics problem for a serial manipulator obtains all possible sets of joint variables that put the EE in a desired pose. This chapter utilizes the equations of the constraint variety for a $2 \mathrm{~A}$-chain derived in Section 4.4 to set-up the systems of equations required to obtain solutions for the inverse kinematics problem for $4 \mathrm{~A}$ - and 6A-manipulators. The focus of this thesis is placed on $6 \mathrm{~A}$-manipulators to match the algorithm presented by Pfurner for 6R-manipulators, discussed in Section 3.2.2, and $4 \mathrm{~A}$-manipulators because this is a useful step towards $6 \mathrm{~A}$-manipulators because it confirms the constraint variety of the $2 \mathrm{~A}$-chains. In both cases the solution is found by intersecting chains of an equal number of joints. Theoretically a similar technique to that presented in this chapter can be applied to chains of three or five joints by intersecting chains of the appropriate number of joints. For example, the solution to a $5 \mathrm{~A}$-chain would involve the intersection of a 3R-chain and 2A-chain. This idea has not been explored in this thesis but should be the subject of future research.

Currently it is not possible to compute a symbolic general solution to the inverse kinematics problem for $4 \mathrm{~A}$ - and $6 \mathrm{~A}$-manipulators because of the complexity and size of the equations, but numerical solutions can be found. This chapter presents the algorithms for the solutions using arbitrary numerical examples.

The algorithm is illustrated for the 4A-manipulator with a detailed example. A numerical example for a $6 \mathrm{~A}$-manipulator is also provided, however the solution could not be completed and remains elusive. The reasons for this and some possible ways to complete 
the solution are discussed.

\subsection{Inverse Kinematics of a 4A-Manipulator}

Examining the inverse kinematics of a $4 \mathrm{~A}$-manipulator is a logical step towards exploring the inverse kinematics of $6 \mathrm{~A}$-manipulators utilizing the kinematic mapping method. With each step of the algorithm the size, degree and complexity of the equations greatly increases, making a general solution to the inverse kinematics problem not computable at this point. Regardless, it is strongly believed that appropriate conditioning of the equations will ultimately reveal a practical and computable version of the algorithm. Therefore the algorithm is presented so that the procedure may be applied to a variety of manipulator configurations. The theory behind the algorithm is presented first followed by a general numeric example presented step-by-step that illustrates the procedure.

\subsubsection{Theory Behind 4A-manipulator Inverse Kinematic Algorithm}

For a known 4A-manipulator the inverse kinematics problem involves obtaining all sets of four joint variables that put the $\mathrm{EE}$ in a target pose, $\Sigma_{\text {target }}$, described by matrix $\mathbf{E E}_{T}$, relative to the base frame. Real solutions exist only if the target pose is within the reachable workspace of the manipulator.

The 4A-chain is broken into two 2A-chains with the theoretical break at the end of the second link. The chain containing the base of the original $4 \mathrm{~A}$-chain is called the left chain and the chain containing the $\mathrm{EE}$ of the original $4 \mathrm{~A}$-chain is the right chain. The 4A-manipulator EE becomes the base of the right chain and is placed at the target pose. A reference frame is established at the break point on each of the left and right chains called $\Sigma_{L}$ and $\Sigma_{R}$ respectively. $\Sigma_{L}$ and $\Sigma_{R}$ are described by matrices $\mathbf{E E}_{L}$ and $\mathbf{E E}_{R}$. The constraint varieties for a canonical $2 \mathrm{~A}$-chain are modified via transformations of the reference frames to represent the non-canonical left and right chains. This results in a set of equations that are a function of only the unknown Study parameters $x_{i}, y_{i}$ for $i \in\{0, \ldots, 3\}$. Solving for the unknowns that satisfy the set of equations provides the Study parameters where the 
constraint varieties of the left and right chains intersect. The solutions represent instances where $\Sigma_{L}$ and $\Sigma_{R}$ are coincident. The joint variables of the left and right chains that put their respective EE frames in that pose are determined and represent the solution to the inverse kinematics problem. This is the basic theory, however each step requires a more detailed explanation.

\section{Breaking the Chain}

The 4A-chain is theoretically broken at the end of the of the second link where it connects to the third joint. When the $4 \mathrm{~A}$-chain is unbroken $\Sigma_{L}$ and $\Sigma_{R}$ are coincident with reference frame $\Sigma_{2}$. This means $\Sigma_{L}$ and $\Sigma_{R}$ are affixed to the 'fixed' base of the GDP making up the third A-joint in the chain, when the 'fixed' base of joint $i$ is considered to be the part of the joint fixed to link $i-1$. Figure 5.1 shows where the theoretical break is made in the 4A-chain to produce the left and right 2A-chains.

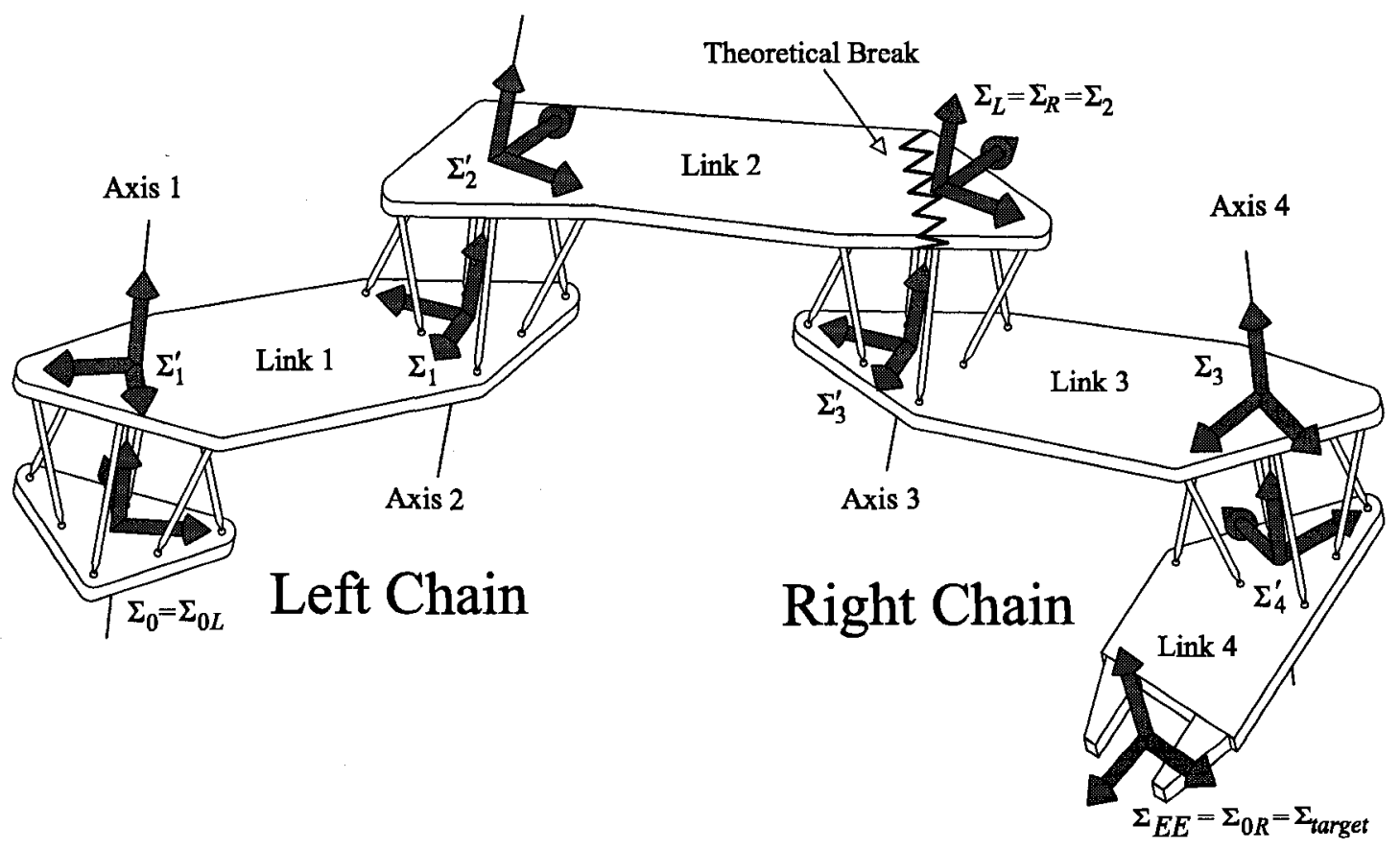

Figure 5.1: 4A-manipulator showing the theoretical break between the left and right 2Achains.

The left chain contains links and joints 1 and 2 . The base reference frame of the left 
chain, $\Sigma_{0 L}$, remains the same as the base reference frame of the original 6A-manipulator, represented by frame $\Sigma_{0}$. The right chain contains the links and joints 3 and 4 . The base reference frame of the right chain, $\Sigma_{0 R}$ is established as the $4 \mathrm{~A}$-manipulator EE in the target pose. $\Sigma_{0 R}$ is known and fixed relative to $\Sigma_{0}$. The order of the joints in the right serial 2A-chain are joints 4 then 3 of the original chain.

With the two $2 \mathrm{~A}$-chains defined it is now possible to obtain the constraint varieties of the left and right $2 \mathrm{~A}$-chains by utilizing the constraint varieties of the canonical $2 \mathrm{~A}$-chain obtained in Section 4.4.2 as a starting point.

\section{Obtaining the Left 2A-chain Constraint Variety}

The assumption that the base of the original $4 \mathrm{R}$-chain was selected such that as many DH-parameters as possible are zero has been made, meaning that the base reference frame of the manipulator is coincident with the universal reference frame. If this is not the case additional transformations in the base reference frame are required. The procedure for this change is similar to that of the right chain as presented in the next subsection, and is not shown here to simplify the equations.

The constraint variety previously obtained for the canonical 2A-chain included only joint 1 , link 1 and joint 2, this means that $i=1$ in the set of equations for the canonical constraint variety. $\Sigma_{L}$ is at the end of link 2 in the left chain, meaning the effect of the second link must be added to the constraint variety in a similar manner as the first link was added to the canonical 1A-constraint variety in Section 4.4.2. The addition of link 2 and $\Sigma_{L}$ is illustrated in Figure 5.2. Link 2 is represented by substitution of the link parameters into Equation (2.16) which, after tangent of the half-angle substitution is

$$
\mathbf{G}_{2}=\left[\begin{array}{cccc}
1 & 0 & 0 & 0 \\
a_{2} & 1 & 0 & 0 \\
0 & 0 & \frac{1-a l_{2}^{2}}{1+a l_{2}^{2}} & -\frac{2 a l_{2}^{2}}{1+a l_{2}^{2}} \\
d_{2} & 0 & \frac{2 a l_{2}^{2}}{1+a l_{2}^{2}} & \frac{1-a l_{2}^{2}}{1+a l_{2}^{2}}
\end{array}\right]
$$




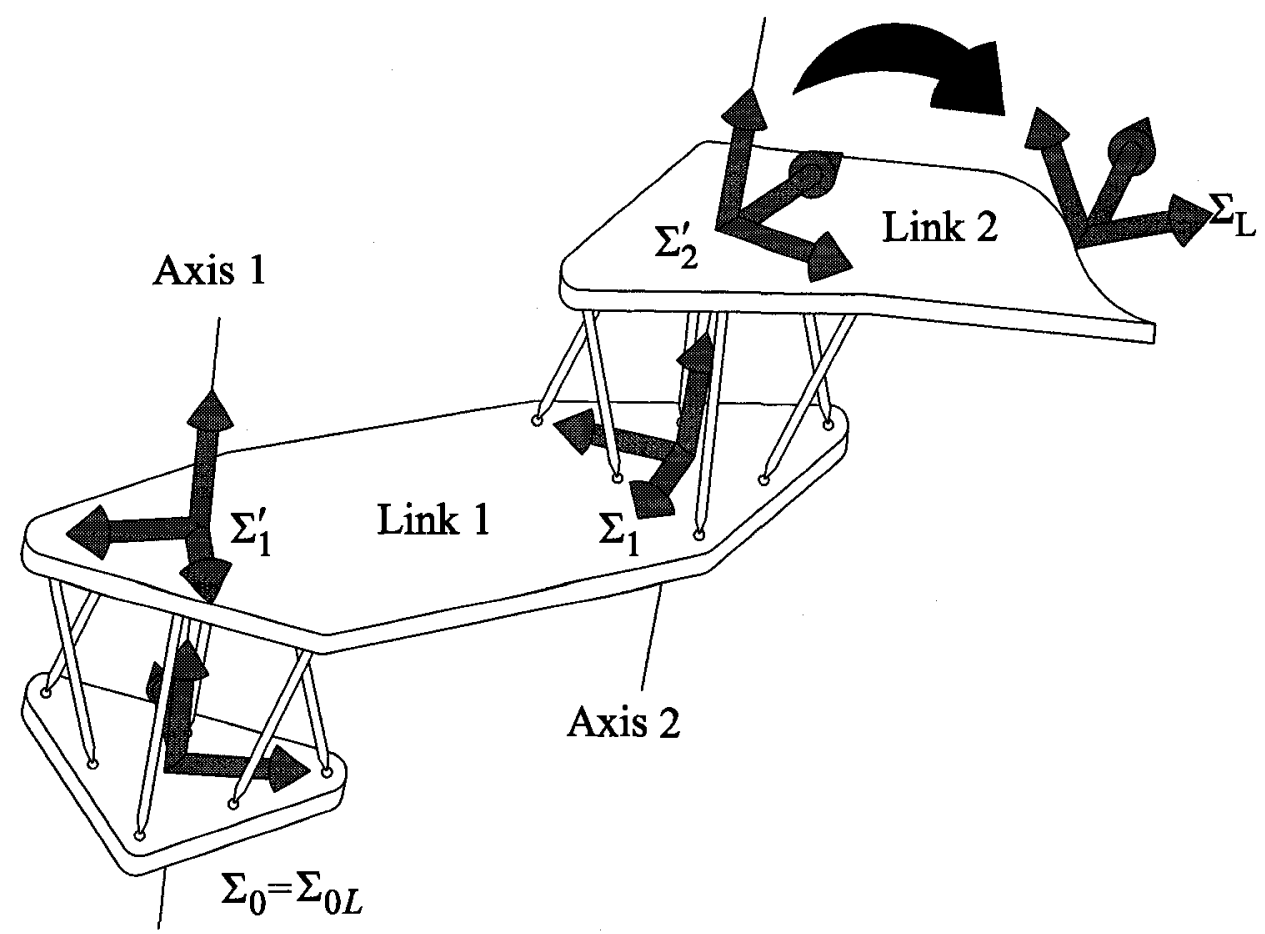

Figure 5.2: 2A-pair illustrating the addition of the second link to the left chain with $\Sigma_{L}$.

After obtaining the Study parameters of matrix $\mathbf{G}_{2}$ and substituting them into $\mathbf{T}_{m}$ of Equation (2.21) the transformed equations of the now non-canonical constraint variety are found by substituting $\mathbf{x}^{\prime}$ for $\mathbf{x}$ in the set of equations of Equation (4.7), where $\mathbf{x}^{\prime}$ is found using Equation (4.6), substituting $\mathbf{G}_{2}$ for $\mathbf{G}_{i}$. The intersection of the resulting set of equations, which includes the unchanged $S_{6}^{2}$, is the constraint variety for the left hand chain.

The equations can be confirmed by substituting in the general Study parameters of $\mathbf{E E}_{L}$. These are obtained by finding the Study parameters of

$$
\mathbf{E E}_{L}=\mathbf{M}_{1} \mathbf{G}_{1} \mathbf{M}_{2} \mathbf{G}_{2}
$$

which is a function of the link parameters and the joint variables $u_{1}$ and $u_{2}$. They must all be identically zero to satisfy the equations of the constraint variety. 


\section{Obtaining the Right 2A-chain Constraint Variety}

The procedure for obtaining the equations that represent the constraint variety of the right chain starts by substituting $-a_{3}$ and $-a l_{3}$ for $a_{1}$ and $a l_{1}$ respectively into the set of equations in Equation (4.7). The negative angles and translations are introduced because when comparing the canonical chain to the right chain, the definitions of the DH-parameters in Section 2.5.1 were in the opposite direction along and about the axes of the reference frames affixed to the links.

Unlike the left chain, it is not possible to assume the base coincides with the base used in the canonical chain and this must be accounted for. To do this, the position of the target EE pose relative to the original base reference frame is accounted for using the target pose matrix $\mathbf{E E}_{T}$ and the fact that the base of the right $2 \mathrm{~A}$-chain is not at the base of the first A-pair, but at the end of the the last link that is defined by the matrix $\mathbf{G}_{4}$. Figure 5.3 shows the addition of the fourth link to the canonical 2A-chain and the position of the right 2A-chain in space relative to the base frame. To get from the original base to the base of the canonical form of the right $2 \mathrm{~A}$-chain the matrix $\mathbf{A}$ is used such that

$$
\mathbf{A}=\mathbf{E E}_{T} \mathbf{G}_{4}^{-1}
$$

The inverse of $\mathbf{G}_{4}$ is used because the link is approached in the opposite direction to how it is defined for the direct kinematics. The Study parameters of the matrix $\mathbf{A}$ are obtained and substituted into the matrix $\mathbf{T}_{b}$ of Equation (2.20). Notice that this time the transformation occurs in the base frame. The transformed equations of the constraint variety are obtained by substituting $\mathbf{x}^{\prime}$ for $\mathbf{x}$ into the set of equations in Equation (4.7), where $\mathbf{x}^{\prime}$ is now found by

$$
\mathbf{x}^{\prime}=\mathbf{T}_{b}(\mathbf{A}) \mathbf{x}
$$

The right chain is not yet fully accounted for because the offset of the third link must be included. In Section 2.5.1 $d_{3}$ is described as the directed distance along axis $\mathrm{Z}_{3}$ and the shift of reference frame is in the opposite direction to which $d_{3}$ was defined, therefore a 


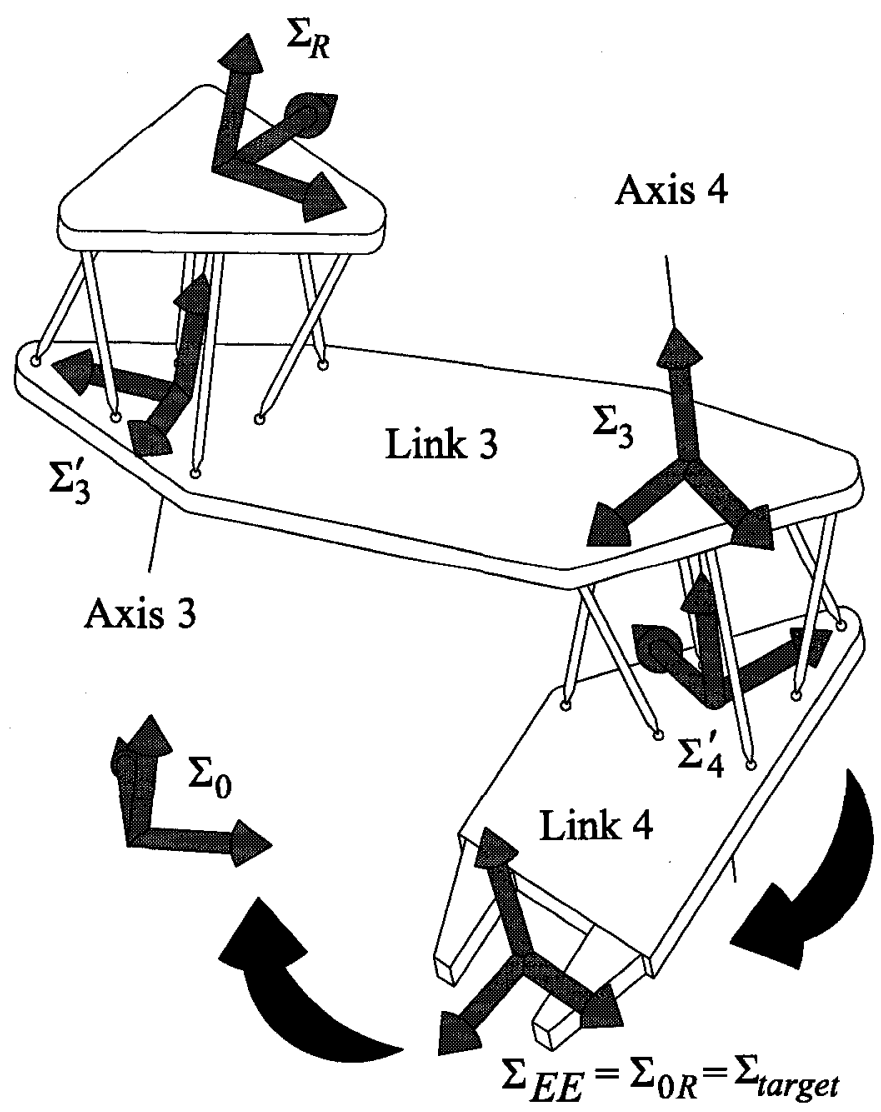

Figure 5.3: 2A-pair illustrating the addition of the fourth link to the canonical 2A-chain in order to obtain the right chain with $\Sigma_{R}$ and the target frame $\Sigma_{E E}$ relative to the base frame $\Sigma_{0}$.

translation of $-d_{3}$ is required. This translation is given by the transformation matrix

$$
\mathbf{G}_{3}^{\prime}=\left[\begin{array}{cccc}
1 & 0 & 0 & 0 \\
0 & 1 & 0 & 0 \\
0 & 0 & 1 & 0 \\
-d_{3} & 0 & 0 & 1
\end{array}\right]
$$

the translation is accounted for by again substituting $\mathbf{x}^{\prime}$ for $\mathbf{x}$ in the set of equations of the previous step where $\mathbf{x}^{\prime}$ is given by Equation (4.6) with $\mathbf{G}_{3}^{\prime}$ substituted for $\mathbf{G}_{i}$. The resulting 
set of equations is the representation of the constraint variety of the right $2 \mathrm{~A}$-chain. This set again contains the unmodified $S_{6}^{2}$ and four additional equations. The intersection of these four equations and the Study quadric is the constraint variety for the right chain.

Confirmation that the set of equations obtained here represents the constraint variety is obtained by substituting the Study parameters of

$$
\mathbf{E E}_{R}=\mathbf{E E}_{T} \mathbf{G}_{4}^{-1} \mathbf{M}_{4}^{-1} \mathbf{G}_{3}^{-1} \mathbf{M}_{3}^{-1}
$$

which are functions of joint variables $u_{3}$ and $u_{4}$, into each of the constraint variety equations to ensure that they are in fact equal to zero, as required.

\section{Intersecting the Constraint Varieties}

The constraint variety of each of the left and right chains is described by the intersection of five equations. In both cases one of those equations is $S_{6}^{2}$. Therefore it can be seen that the intersection of the left and right constraint varieties is the intersection of a set of eight equations with $S_{6}^{2}$. The unknowns in the set of nine equations are the eight homogenous Study parameters, of which one unknown can be set equal to unity, such as $x_{0}=1$, since the exceptional generator $x_{0}=x_{1}=x_{2}=x_{3}=0$ is excluded. This yields an over determined set of nine equations in eight unknowns. In general there are no solutions to this system, but due to the method of obtaining this set of equations there is at least one real solution for a target pose that is within the $4 \mathrm{~A}$-manipulator workspace.

The method for obtaining the solutions to the set of equations is more easily shown through an example and is described in detail in Section 5.1.2. The general procedure requires examining the structure of the equations and eliminating variables in the most logical way, starting with linear terms and working towards a univariate polynomial using seven of the nine equations. The solutions of this polynomial are obtained and backsubstituted to obtain a set of variables that can be tested in the remaining two equations. Those sets of Study parameters that satisfy all equations describe instances where $\Sigma_{L}$ and $\Sigma_{R}$ are coincident and the $4 \mathrm{~A}$-manipulator is a continuous kinematic chain with the EE in 
the target pose.

\section{Obtaining the Joint Variables}

The sets of Study parameters obtained from the intersection of the left and right constraint varieties describe the pose of the coincident reference frames $\Sigma_{L}$ and $\Sigma_{R}$ relative to $\Sigma_{0}$. In order to obtain the four joint angles of the 4A-manipulator the inverse kinematic problem for the two 2A-chains must be solved. For the left chain this can be done as follows. Obtaining the Study parameters of the result of Equation (5.1) provides eight functions in terms of the two joint variables $u_{1}$ and $u_{2}$, and can be normalized by dividing each equation by one of the others. It is useful to divide by the equation corresponding Study parameter that was used for normalization during the intersection of the constraint manifolds. The equations are now set equal to the set of Study parameters obtained in the previous step for the left chain, resulting in a set of seven equations in two unknowns. Once again, in general there is no solution, but due to the nature of the problem at least one real solution exists if a real solution was obtained when intersecting the constraint varieties. Solving two of the equations provides many solutions which are tested in the remaining five equations. The sets of joint variables that satisfy all seven equations are the solution. It is widely believed that like 2R-chains there is in general only one solution.

The right chain provides the joint variables $u_{3}$ and $u_{4}$. The method of the left chain can be applied after some initial pre-processing. Using the matrix of Equation (2.11) the $4 \times 4$ transformation matrix $\mathbf{T}_{E E_{R}}$ describing $\Sigma_{R}$ relative to $\Sigma_{0}$ can be constructed using the Study parameters found in the solution to the intersection problem. $\Sigma_{E E}$ relative to $\Sigma_{R}$ is given by

$$
\mathbf{E E}_{\mathrm{T}-\text { relative }}=\mathbf{T}_{E E_{R}}^{-1} \mathbf{E E}_{T}
$$

where $\mathbf{E E}_{\mathrm{T}-\text { relative }}$ is the matrix describing the pose of $\Sigma_{E E}$ relative to $\Sigma_{R}$. The transformation from $\Sigma_{R}$ to $\Sigma_{E E}$ given by

$$
\mathbf{T}_{R}=\mathbf{M}_{3} \mathbf{G}_{3} \mathbf{M}_{4} \mathbf{G}_{4}
$$


is a function of $u_{3}$ and $u_{4}$. Obtaining the Study parameters of $\mathbf{E E}_{\mathrm{T}-\text { relative }}$ and the $\mathbf{T}_{R}$ and normalizing produces a set of seven equations and two unknowns that can be solved in a similar manner to left chain yielding the joint variables $u_{3}$ and $u_{4}$.

With the joint variables $u_{i}, i \in\{1, \ldots, 4\}$ obtained for every intersection point between the left and right constraint varieties, the solution to the inverse kinematic problem has been found.

\subsubsection{Numeric Example of Inverse Kinematics for a 4A-manipulator}

The procedure for solving the $4 \mathrm{~A}$-manipulator inverse kinematics problem is demonstrated with a numeric example. The $\mathrm{DH}$-parameters of Table 5.1 describe the geometry of the manipulator using generic dimensions.

Table 5.1: DH-parameters for 4A-manipulator inverse kinematics numeric example.

\begin{tabular}{|c||c|c|c|}
\hline link $i$ & $a_{i}$ & $a l_{i}$ & $d_{i}$ \\
\hline \hline 1 & 3 & $\frac{1}{3}$ & 0 \\
\hline 2 & 1 & 2 & $\frac{2}{3}$ \\
\hline 3 & $\frac{1}{2}$ & $\frac{3}{2}$ & 3 \\
\hline 4 & 4 & 3 & 2 \\
\hline
\end{tabular}

\section{Obtaining the Target EE pose}

To ensure the target pose is within the workspace of the manipulator a set of joint variables is arbitrarily selected and the direct kinematics equations are used to determine the pose. The joint variables selected are shown in Table 5.2. This also allows the results of the inverse kinematic algorithm to be confirmed. After obtaining the target pose these joint variables are not used again until they are compared with the results of the algorithm. Note that these are the angles after tangent of the half-angle substitution, $u_{i}$, not $\theta_{i}$. The target pose is now obtained using the matrix equation

$$
\mathbf{T}_{\mathrm{EE}}=\mathbf{M}_{1} \mathbf{G}_{1} \mathbf{M}_{2} \mathbf{G}_{2} \mathbf{M}_{3} \mathbf{G}_{3} \mathbf{M}_{4} \mathbf{G}_{4}
$$


Table 5.2: Target joint variables for 4A-manipulator inverse kinematics numeric example.

\begin{tabular}{|c||c|}
\hline joint $i$ & $u_{i}$ \\
\hline \hline 1 & $\frac{1}{2}$ \\
\hline 2 & -2 \\
\hline 3 & $\frac{3}{2}$ \\
\hline 4 & $\frac{1}{3}$ \\
\hline
\end{tabular}

which results in

$$
\mathbf{T}_{\mathbf{E E}}=\left[\begin{array}{cccc}
1 & 0 & 0 & 0 \\
-\frac{5972535403}{1716406250} & -\frac{288513461}{858203125} & \frac{3685386792}{4291015625} & -\frac{1658233656}{4291015625} \\
\frac{7789023548}{858203125} & \frac{670726152}{858203125} & \frac{109229481}{4291015625} & -\frac{2674704608}{4291015625} \\
-\frac{209924864}{102984375} & -\frac{18039912}{34328125} & -\frac{87807136}{171640625} & -\frac{116680977}{171640625}
\end{array}\right]
$$

Now the joint angles used to create the target pose are essentially 'forgotten' and the goal is to obtain all the sets of joint angles which will put the end effector of this manipulator in this pose.

\section{Left 2A-chain}

The 4A-chain is broken as described in Section 5.1.1 to create the left and right chain. For the left chain the constant DH-parameters $a_{1}$ and $a l_{1}$ can be directly substituted into the set of equations in Equation (4.7). The study parameters of $\mathbf{G}_{2}$ are obtained as per Section 2.4.6 and the $8 \times 8$ transformation matrix $\mathbf{T}_{m}\left(\mathbf{G}_{2}\right)$ is constructed. $\mathbf{x}^{\prime}$ is found using Equation 4.6 with $i=2$ and substituted into the set of equations for the canonical 2A-chain. The resulting set of equations describing the constraint variety for the left $2 \mathrm{~A}$-chain is provided in Appendix B.1. 


\section{Right 2A-chain}

The DH-parameters $-a_{3}$ and $-a l_{3}$ are substituted into the set of equations in Equation (4.7) for $a_{1}$ and $a l_{1}$ respectively. The Study parameters of the $\mathbf{A}$, found using Equation (5.2) are used to build $\mathbf{T}_{b}(\mathbf{A})$ and substitution of $\mathbf{x}^{\prime}$ from Equation (5.3) into the canonical set of equations yields an intermediate set of equations. The effect of matrix $\mathbf{G}_{3}^{\prime}$ of Equation (5.4) is added in the moving frame using $\mathbf{T}_{m}\left(\mathbf{G}_{3}^{\prime}\right)$ to produce the final set of equations whose intersection yields the constraint variety for the right 2A-chain which is is provided in Appendix B.2.

\section{Intersecting the Constraint Varieties}

Intersecting the left and right constraint varieties requires finding the intersection of the two sets of five equations from each of the left and right chains, with $S_{6}^{2}$ being common to both. The solutions to the inverse kinematics problem requires solving the set of nine equations for the eight homogenous unknown Study parameters.

The individual equations of the left constraint variety are referred to as $L_{1}, L_{2}, L_{3}, L_{4}$ and $L_{5}$ in the same order as they are defined in the set of equations in Appendix B.1 and for the right constraint variety $R_{1}, R_{2}, R_{3}, R_{4}$ and $R_{5}$ in the same order as they are defined in the set of equations in Appendix B.2. Note that $L_{1}=R_{1}=S_{6}^{2}$. The following algorithm is based on examination of the set of equations for this example. This exact procedure may or may not easily apply to other manipulator configurations, but the general procedure may be similar.

The intermediate equations obtained during the solution are based on the equations of the constraint varieties in Appendices B.1 and B.2. These intermediate equations are described but not shown. Since the Study parameters are homogenous the solution begins by setting $x_{0}=1$. It is then noted that $L_{3}$ and $R_{3}$ both contain an $x_{1}^{2}$ term. This term can be eliminated from $L_{3}$ using $L_{3} F-R_{3}$, where $F$ is the coefficient of $x_{1}^{2}$ in $R_{3}$ divided by the coefficient of $x_{1}^{2}$ in $L_{3}$, yielding an equation, $L R_{3}$, that is linear in $x_{1}$. Solving $L R_{3}$ for the linear term gives $x_{1}$ as a function of $x_{2}$ and $x_{3}$. Equations $L_{2}$ and $R_{2}$ are already linear in 
terms of $y_{0}$ and $y_{1}$ so they are simultaneously solved for the linear terms to give $y_{0}$ and $y_{1}$ in terms of $x_{2}, x_{3}, y_{2}$ and $y_{3}$ after the previous solution for $x_{1}$ is included. Substitution of these solutions into $S_{6}^{2}$ provides an equation linear in $y_{2}$. Solving for this linear term gives $y_{2}$ as a function of $x_{2}, x_{3}$ and $y_{3}$. Further manipulation does not reveal any more linear terms. Substituting the results so far into $L_{4}$ and $R_{4}$ and using the resultant method to eliminate $y_{3}$ provides a large equation $L R_{4}$. With a new equation $l r_{3}$ (different than $L R_{3}$ ) defined as the greatest common divisor of $L_{3}$ and $L_{4}$ after substitutions, the resultant method is used to eliminate $x_{2}$ from $L R_{4}$ and $l r_{3}$, providing a univariate polynomial in $x_{3}$. The polynomial has seven factors, they are $\left(71 x_{3}+72\right),\left(125467281 * x_{3}^{2}-226420416 * x_{3}+266606569\right)^{2}$, $\left(217813969 * x_{3}^{2}+226420416 * x_{3}+76674681\right)^{2},\left(89578187453833 * x_{3}^{2}+91854687203712 *\right.$ $\left.x_{3}+32320518819417\right)^{2},\left(x_{3}^{2}+1\right)^{2},\left(293521+3144728 * x_{3}\right)^{32}$ and the last factor is of degree 48. These factors can be solved for several numeric values of $x_{3}$, but by inspecting the results it has been found that only one result also satisfies the remaining two equations $L_{5}$ and $R_{5}$. This single solution means that there is only one solution to the inverse kinematics problem for this specific example. It has not been generally proven that there is only one solution to the $4 \mathrm{~A}$-manipulator inverse kinematics problem, however the fact that the general $4 \mathrm{R}$-manipulator has only one solution suggests it may be true.

The valid solution yields $x_{3}=-\frac{72}{71} \doteq-1.014084504$, meaning that the only factor to produce meaningful results for this example is $\left(71 x_{3}+72\right)$. Substitution into $l r_{3}$ yields two solutions for $x_{2}$ but the only one that proves to be valid is $x_{2}=-\frac{144}{71} \doteq-2.028169022$. Once again two solutions are obtained for $y_{3}$ when the results are substituted into either $L_{4}$ or $R_{4}$, but $y_{3}=-\frac{415}{213} \doteq-1.948356835$ is the only solution that proves to be valid in the entire set of equations. The remaining unknowns are found linearly to produce the set of 
study parameters

$$
\left(\begin{array}{l}
x_{0} \\
x_{1} \\
x_{2} \\
x_{3} \\
y_{0} \\
y_{1} \\
y_{2} \\
y_{3}
\end{array}\right)=\left(\begin{array}{c}
1 \\
\frac{17}{71} \\
-\frac{144}{71} \\
-\frac{72}{71} \\
-\frac{230}{71} \\
\frac{50}{71} \\
-\frac{115}{213} \\
-\frac{415}{213}
\end{array}\right) \doteq\left(\begin{array}{c}
1 \\
0.2394366165 \\
-2.028169022 \\
-1.014084504 \\
-3.239436633 \\
0.7042253376 \\
-0.5399060961 \\
-1.948356835
\end{array}\right) .
$$

This set of Study parameters describes the pose of the coincident frames $\Sigma_{L}$ and $\Sigma_{R}$ relative to $\Sigma_{0}$. The joint variables of the left and right chains that put the frames in that pose must now be obtained.

\section{Solving for the Joint Variables}

To obtain the joint variables from the left chain the matrix representation of the direct kinematic equations is found using Equation (5.1) and the Study parameters of that matrix, which are functions of $u_{1}$ and $u_{2}$, are normalized by dividing each equation by the first and then set equal to the Study parameters of $\Sigma_{L}$. These equations are shown in Appendix B.3. From this procedure a set of seven equations in two unknowns is obtained. Two equations are used to solve numerically for $u_{1}$ and $u_{2}$ providing many solutions. Each solution is tested in the remaining five equations. The only set of joint variables to satisfy the entire set of equations is $\left\{u_{1}=0.5000000020, u_{2}=-2.000000013\right\}$.

To obtain the right chain the $4 \times 4$ matrix defining the pose of $\Sigma_{R}$ is required and is obtained using Equation (2.11), as are the Study parameters that were obtained by 
intersecting the left and right constraint varieties. This matrix is called $\mathbf{T}_{E E_{R}}$ and

$$
\mathbf{T}_{E E_{R}}=\left[\begin{array}{cccc}
1.0000 & 0.0 & 0.0 & 0.0 \\
-1.5756 & -0.6588 & 0.1704 & -0.7326 \\
2.3737 & -0.4838 & 0.6497 & 0.5863 \\
1.2693 & 0.5760 & 0.7407 & -0.3456
\end{array}\right]
$$

The pose of $\Sigma_{E E}$ relative to $\Sigma_{R}$ is found by Equation (5.6) producing matrix $\mathbf{E E}_{\mathrm{T}-\text { relative }}$. The direct kinematics of the right chain are found using Equation (5.7) to produce matrix $\mathbf{T}_{R}$. The Study parameters of $\mathbf{T}_{R}$ are a function of joint variables $u_{3}$ and $u_{4}$ and are normalized by dividing by the first equation of the set. These functions are then set equal to the normalized Study parameters of $\mathbf{E E}_{\mathrm{T}-\text { relative }}$ to produce an over constrained set of seven equations in two unknowns. These equations are shown in Appendix B.4 and because of how the equations were obtained there is at least one solution. The procedure for solving this over constrained set of equations is similar to that of the left chain and produces the set of joint variables $\left\{u_{3}=1.500000001, u_{4}=0.3333333353\right\}$.

The joint variables obtained using this algorithm match the original joint variables of Table 5.2. The small differences are brought about by the numerical accuracy of the computer and software used. Table 5.3 shows the start values of the joint parameters compared with the results obtained using the algorithm.

Table 5.3: Comparison of target joint variables with algorithm results for 4A-manipulator inverse kinematics numeric example.

\begin{tabular}{|c||c|c|}
\hline joint $i$ & Target & Algorithm \\
\hline \hline 1 & $\frac{1}{2}$ & 0.5000000020 \\
\hline 2 & -2 & -2.000000013 \\
\hline 3 & $\frac{3}{2}$ & 1.500000001 \\
\hline 4 & $\frac{1}{3}$ & 0.3333333353 \\
\hline
\end{tabular}




\subsection{Inverse Kinematics of a 6A-Manipulator}

The algorithm for solving the inverse kinematics problem for a general 6A-manipulator parallels that of the 4A-manipulator, but is more complicated. Though the algorithm is similar, the size of the equations makes the actual solution to the problem much more difficult to obtain, both in the general case and for a specific example.

The theory behind the algorithm is presented in the first part of this section, followed by a numerical example. The example is not taken to completion due to the prohibitive size of the equations. The derivation of the equations is shown and the results of some of the attempts at a solution are discussed.

\subsubsection{Theory Behind the 6A-manipulator Inverse Kinematic Algorithm}

The basic theory behind the $6 \mathrm{~A}$-manipulator inverse kinematics algorithm is very similar to that of the 4A-manipulator. For any given target EE pose the manipulator is theoretically broken into a left and right chain, the base and EE reference frame for each chain is established, the equations describing the constraint variety are obtained for each chain, the constraint varieties are intersected and the inverse kinematics for the left and right chains produces the sets of joint variables that place the $\mathrm{EE}$ in the target pose, if it is in the manipulator workspace.

The major differences from the 4A-algorithm stem from the fact that the left and right chains are now 3A-chains. This requires the addition of a third joint variable to the constraint varieties. This is done in a manner similar to adding the second joint variable to the single A-pair constraint variety as was done in Section 4.4.2, but without eliminating the newly introduced joint variable. This means that when intersecting the constraint varieties a set of nine equations in nine unknowns - the seven normalized Study parameters and a joint variable from each of the left and right chains - needs to be solved. The details of each step to achieve this is described in the following subsections. 


\section{Breaking the Chain}

The 6A-chain is theoretically broken at the end of the third link where it meets the fourth joint axis. The EE reference frames of the left and right chain, $\Sigma_{L}$ and $\Sigma_{R}$ respectively, are coincident with $\Sigma_{3}$ when the full $6 \mathrm{~A}$-chain is unbroken. They are located on the base of the fourth joint with the $z$-axes are along the fourth joint axis, the $x$-axes are parallel to the common normal of the third and fourth joint axes and the $y$-axes complete the right-handed reference frame. Figure 5.4 shows where the theoretical break is made in the $6 \mathrm{~A}$-chain to produce the left and right $3 \mathrm{~A}$-chains.

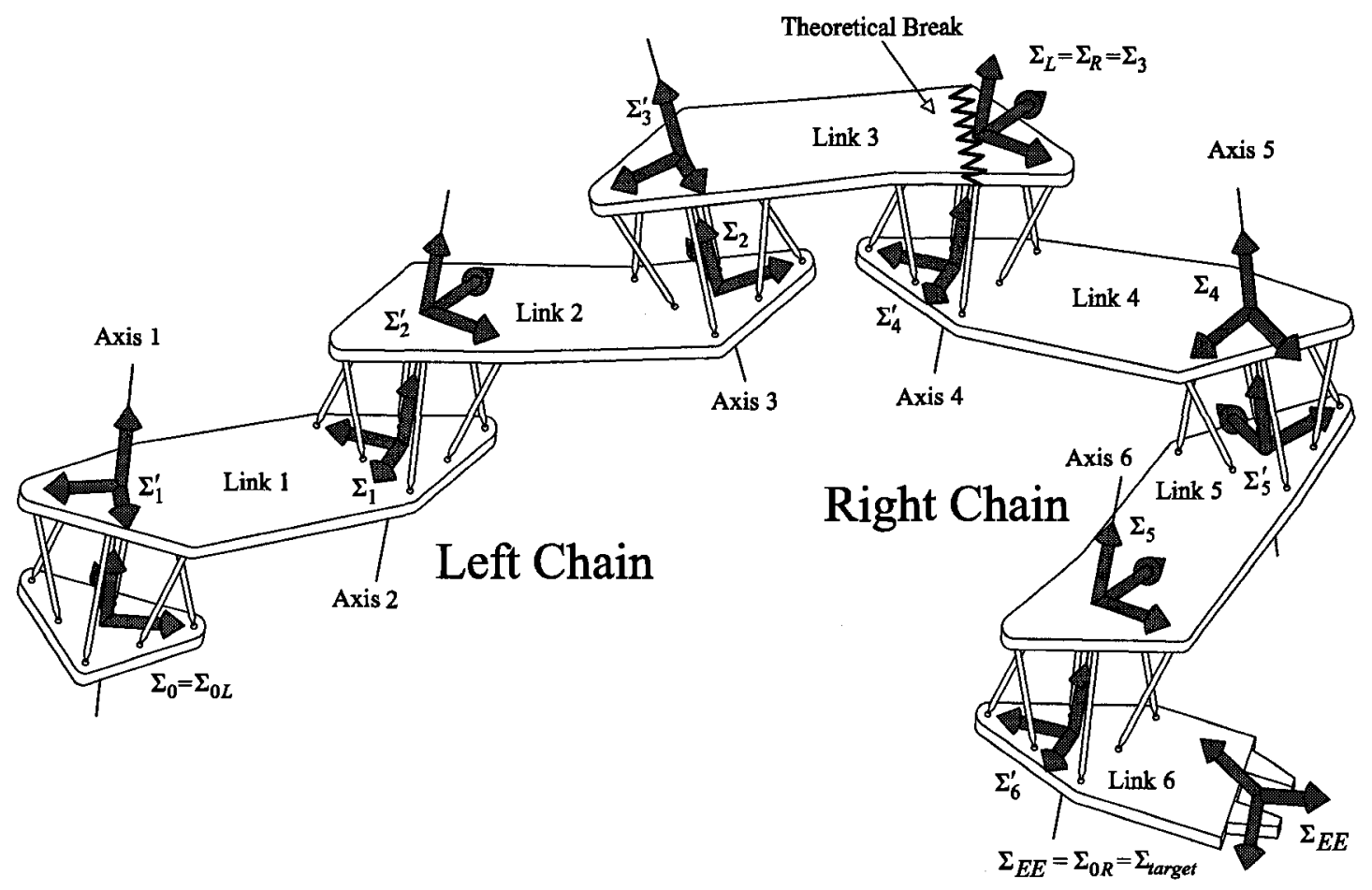

Figure 5.4: 6A-manipulator showing the theoretical break between the left and right 3Achains.

The left chain contains joints 1,2 and 3 and maintains the original $6 \mathrm{~A}$-manipulator base reference frame $\Sigma_{0}$, now called $\Sigma_{0 L}$. The right chain contains joints 4,5 and 6 . The base reference frame of the right chain, $\Sigma_{0 R}$, is established with the $\mathrm{EE}$ of the original $6 \mathrm{~A}$ manipulator in the target EE pose, $\Sigma_{\text {target }} . \Sigma_{0 R}$ is known and fixed relative to $\Sigma_{0}$. When obtaining the constraint variety of the right chain, the order of the joints from the base $\Sigma_{0 R}$ 
to $\Sigma_{R}$ is 6,5 then 4 .

\section{Obtaining the Left 3A-chain Constraint Variety}

The first steps in obtaining the constraint variety for the left 3A-chain are identical to the procedure for the left $2 \mathrm{~A}$-chain of Section 5.1.1. That is, the second link is accounted for by obtaining the Study parameters of $\mathbf{G}_{2}$, building $\mathbf{T}_{m}\left(\mathbf{G}_{2}\right)$ and substituting $\mathbf{x}^{\prime}$ of Equation (4.6) with $i=2$ into the set of equations in Equation (4.7) for $\mathbf{x}$.

The next step is to introduce the third joint variable. This is done using the Study parameters of matrix $\mathbf{M}_{3}$ built using Equation (4.2) with $i=3$. From these, the matrix $\mathbf{T}_{m}\left(\mathbf{M}_{3}\right)$ is built. The terms of this $8 \times 8$ matrix are functions of the joint variable $u_{3}$. The equations obtained for the non-canonical 2A-chain of the previous step are now transformed by substituting in a new $\mathbf{x}^{\prime}$ for $\mathbf{x}$, where $\mathbf{x}^{\prime}$ is obtained from Equation (4.6) with $\mathbf{M}_{3}$ in place of $\mathbf{G}_{i}$. This new set of equations now includes the joint variable $u_{3}$. The third link must also be added to complete the $3 \mathrm{~A}$-chain. This is done using $\mathbf{G}_{3}$ and $\mathbf{T}_{m}\left(\mathbf{G}_{3}\right)$ to obtain $\mathbf{x}^{\prime}$ via Equation (4.6) with $i=3$ and substituting $\mathbf{x}^{\prime}$ for $\mathbf{x}$ in the newest set of equations. Figure 5.5 illustrates the addition of the third joint and third link.

The intersection of the resulting set of equations represents the constraint variety of the left $3 \mathrm{~A}$-chain. These equations are a function of the joint variable $u_{3}$, with the exception of $S_{6}^{2}$ which remains unchanged. This means that the intersection of the equations moves on the Study quadric as the third joint is actuated. It would now be possible to eliminate $u_{3}$ to create the constraint variety of the 3A-chain independent of the joint variable, as was done in Section 4.4.2 to create the 2A-chain constraint variety from the single A-pair constraint variety, however as will be seen it proves more useful to the inverse kinematics algorithm to maintain the current set of equations.

\section{Obtaining the Right 3A-chain Constraint Variety}

The setup of the constraint variety for the right chain 3A-chain again parallels that of the 2A-chain in Section 5.1.1 with the addition of a third joint, similar to the left 3A-chain. The initial procedure is identical to the right 2A-chain. Obtain $\mathbf{A}$ by Equation (5.2) with 


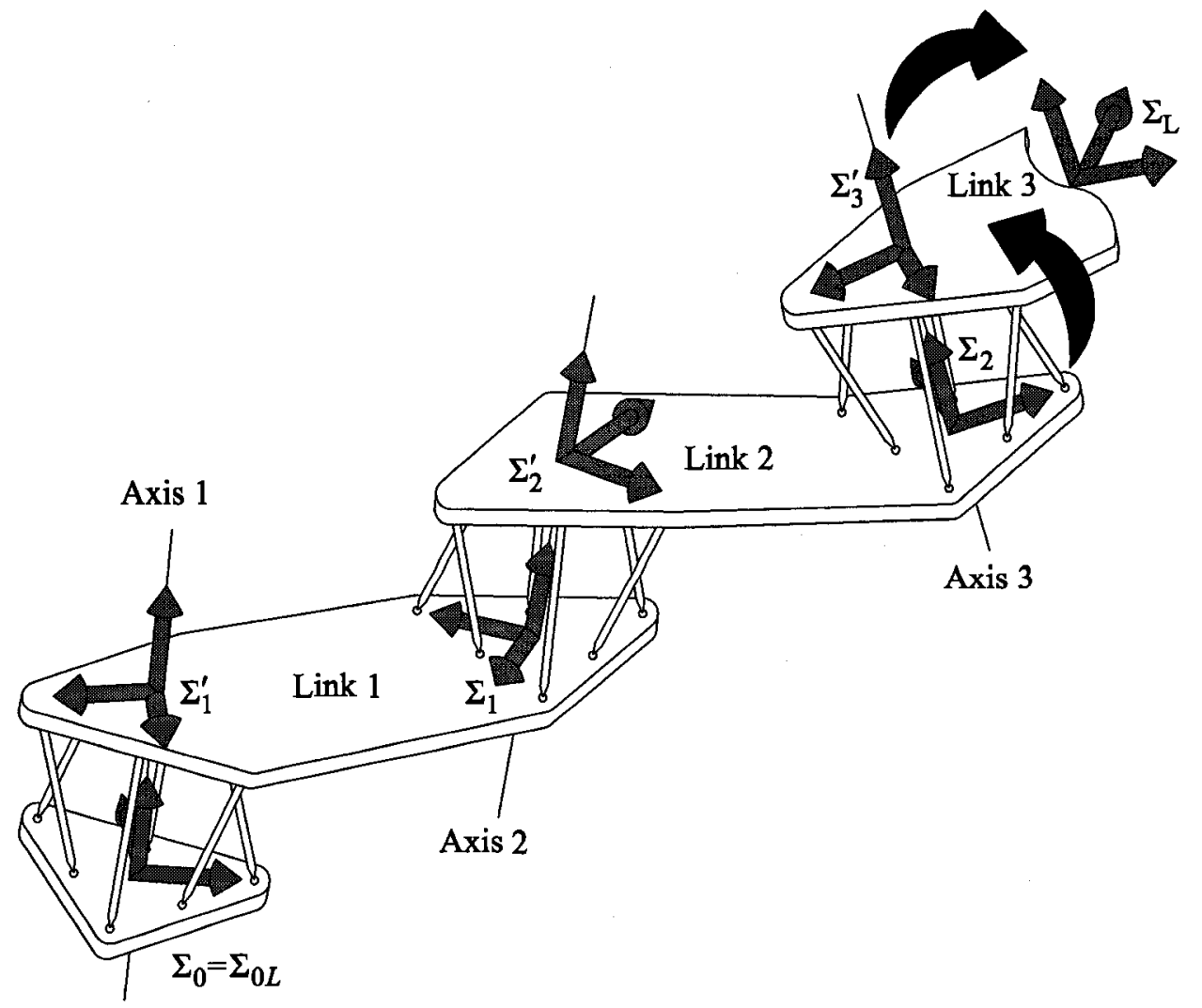

Figure 5.5: 3A-pair illustrating the addition of the third joint and third link to the left chain with $\Sigma_{L}$.

$\mathbf{G}_{6}^{-1}$ replacing $\mathbf{G}_{4}^{-1}$, find the Study parameters of $\mathbf{A}$, build $\mathbf{T}_{b}(\mathbf{A})$, use Equation (5.3) to obtain $\mathbf{x}^{\prime}$ and substitute for $\mathbf{x}$ in the canonical $2 \mathrm{~A}$-chain constraint variety set of equations in Equation (4.7) and setting $a_{1}$ and $a l_{1}$ to $-a_{5}$ and $-a l_{5}$ respectively. Similarly the joint offset of the second joint is accounted for using $\mathbf{G}_{5}^{\prime}$ which is found by Equation (5.4) with $i=5$ substituted for $i=3$. The procedure for transforming the set of equations describing the constraint variety is the same, using $\mathbf{T}_{m}\left(\mathbf{G}_{5}^{\prime}\right)$ in the moving frame.

It is now necessary to introduce the third link in the right chain and the third joint variable, in this case link 4 and $u_{4}$ respectively. To introduce link 4 , the matrix $\mathbf{G}_{4}^{\prime}$ is used. This matrix is based on Equation (4.5) with $i=4$, however $a_{4}, a l_{4}$ and $d_{4}$ are replaced with $-a_{4},-a l_{4}$ and $-d_{4}$, respectively. The change in sign results from the fact that the $\mathrm{EE}$ of the $6 \mathrm{~A}$-manipulator is now the base of the right $3 \mathrm{~A}$-chain and the links are being used in 
the opposite direction with the same original reference frames. The current set of equations describing the constraint variety is modified as before, this time with $\mathbf{T}_{m}\left(\mathbf{G}_{4}^{\prime}\right)$. Joint variable $u_{4}$ is then introduced using the matrix $\mathbf{T}_{m}\left(\mathbf{M}_{4}\right)$ and the method of substitution employed previously. The resulting set of five equations includes $S_{6}^{2}$ and four other equations which are a function of the joint variable $u_{4}$. The set of equations is left in terms of $u_{4}$ as this form is useful in later steps of the inverse kinematics algorithm. Figure 5.6 illustrates the addition of link 4 and joint 4 to the right chain to build a $3 \mathrm{~A}$-chain

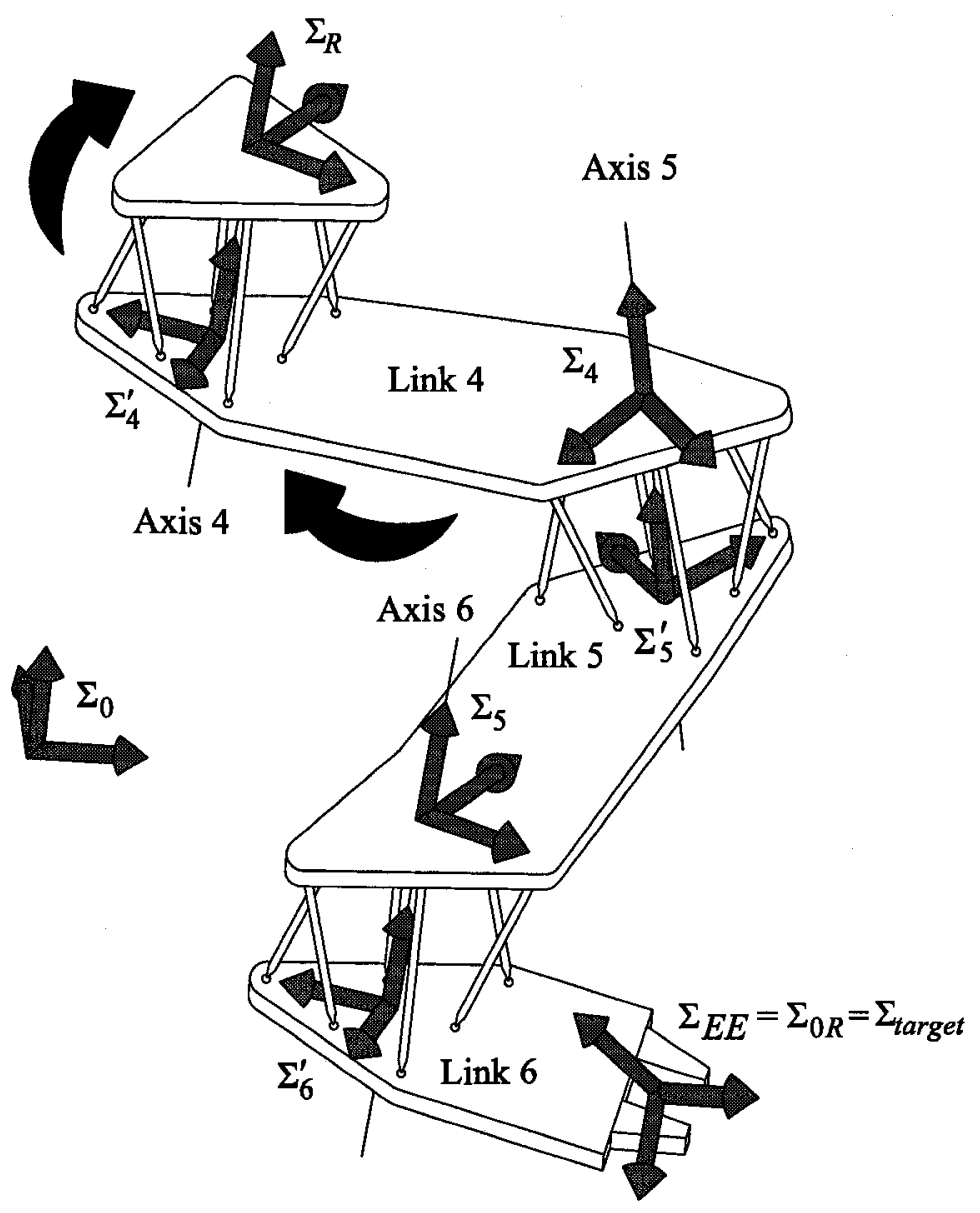

Figure 5.6: 3A-pair illustrating the addition of joint 4 and link 4 to the left chain with $\Sigma_{L}$.

Once again the set of equation provides four equations that intersect on $S_{6}^{2}$ to provide 
the constraint variety of the given 3A-chain. Because the set of equations is function of $u_{4}$ the intersection of the equations moves on $S_{6}^{2}$ as the fourth joint is actuated.

\section{Intersecting the Constraint Varieties}

The left and right $3 \mathrm{~A}$-chains provide constraint varieties that lie on $S_{6}^{2}$ and vary with changes to the joint variables $u_{3}$ and $u_{4}$ respectively. With the same reasoning as for the 4A-manipulator, the 6A-manipulator is unbroken when the constraint variety of the left chain intersects the constraint variety of the right. That is, $\Sigma_{L}$ and $\Sigma_{R}$ are coincident when the set of Study parameters and two joint variables satisfy all of the equations describing constraint varieties of both the left and right chains.

The sets of equations describing the constraint varieties of the left and right chains each contain five equations that are functions of the eight Study parameters and one joint variable $u_{3}$ or $u_{4}$. Since both constraint varieties lie on $S_{6}^{2}$, which is common to both sets of equations, the intersection of the left and right constraint varieties is, after setting one of the homogeneous Study parameters equal to one (for this example, $x_{0}=1$ ), represented by a set of nine equations in the nine unknowns $x_{1}, x_{2}, x_{3}, y_{0}, y_{1}, y_{2}, y_{3}, u_{3}$ and $u_{4}$. The solutions, if any exist, to this fully constrained set of equations represent the points of intersection of the left and right constraint manifolds. Any given solution to the set of equations provides the two joint variables $u_{3}$ and $u_{4}$ and the set of Study parameters that describe the pose of the coincident reference frames $\Sigma_{L}$ and $\Sigma_{R}$. Only target EE poses within manipulator workspace produce real solutions.

The number of intersection points represents the number of solutions to the inverse kinematics problem. Each independent set of solutions provides a different set of joint angles that put the 6A-manipulator in the target EE pose.

In practice, the size of the equations involved prove to be an issue when it comes to solving them. These issues are discussed in the numeric example of Section 5.2.2. 


\section{Obtaining the Remaining Joint Variables}

The procedure for obtaining the remaining four joint angles in the $6 \mathrm{~A}$-chain parallels the method for the 4A-chain and requires the solution to the inverse kinematics of two $2 \mathrm{~A}$-chains. The following procedure is utilized for each independent set of eight Study parameters and two joint variables obtained by intersecting the left and right constraint varieties.

The left chain contains the joint variables $u_{1}, u_{2}$ and $u_{3}$, with the later already determined for each solution set. The pose of reference frame $\Sigma_{L}$ relative to $\Sigma_{0}$ is given in $4 \times 4$ matrix form by

$$
\mathbf{E E}_{L}=\mathbf{M}_{1} \mathbf{G}_{1} \mathbf{M}_{2} \mathbf{G}_{2} \mathbf{M}_{3} \mathbf{G}_{3}
$$

and is a function of the joint variables $u_{1}, u_{2}$ and $u_{3}$. For any solution set $u_{3}$ is already known and can be substituted in at this time. The Study parameters of $\mathbf{E E}_{L}$ as a function of $u_{1}$ and $u_{2}$ are obtained and normalized by dividing all the equations by the first equation then set equal to the corresponding Study parameters of the solution set. This yields seven equations in terms of the two unknowns $u_{1}$ and $u_{2}$. The procedure used to solve this over determined system of equations is now the same as for the left $2 \mathrm{~A}$-chain of the 4A-manipulator inverse kinematics and is described in Section 5.1.1.

The right chain contains the joint variables $u_{4}, u_{5}$ and $u_{6}$, with $u_{4}$ already determined for each solution set. Like in the case of the 4A-manipulator the inverse kinematics of the right $2 \mathrm{~A}$-chain requires some preprocessing before the joint variables can be obtained using the same techniques as the left chain. The matrix $\mathbf{T}_{E E_{R}}$ represents the pose of $\Sigma_{R}$ relative to $\Sigma_{0}$ and is found by substituting the Study parameters of the solution set into Equation (2.11). Using this, the pose of the target $6 \mathrm{~A}$-manipulator EE relative to $\Sigma_{R}$, represented

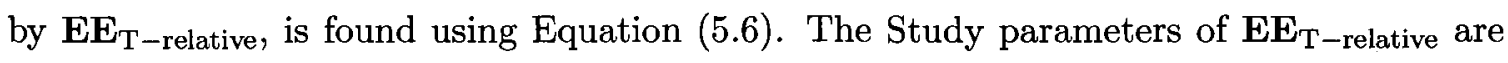
computed as per Section 2.4.6. In terms of the joint variables, the transformation from $\Sigma_{R}$ to $\Sigma_{E E_{T}}$ is given by

$$
\mathbf{T}_{R}=\mathbf{M}_{4} \mathbf{G}_{4} \mathbf{M}_{5} \mathbf{G}_{5} \mathbf{M}_{6} \mathbf{G}_{6}
$$

which is a function of $u_{5}$ and $u_{6}$ after substitution of the known $u_{4}$ from the solution 
set. Obtaining the study parameters of $\mathbf{T}_{R}$, normalizing and setting equal to the Study

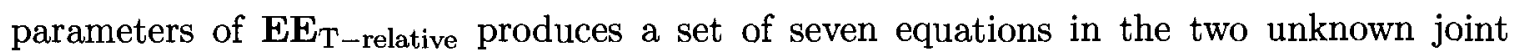
variables $u_{5}$ and $u_{6}$. The algorithm for solving this over constrained set of equations is given in Section 5.1.1 and because of the way the equations were obtained there is a real solution.

When the entire set of six joint variables have been obtained for each intersection point of the left and right constraint varieties the inverse kinematics problem of the $6 \mathrm{~A}$-manipulator can be said to be solved for the given target $\mathrm{EE}$ pose. The number of real and complex solutions is dependent on the intersection points of the constraint varieties.

\subsubsection{Numeric Example of Inverse Kinematics for a 6A-manipulator}

It is useful to use a numeric example to illustrate the techniques and issues presented in the previous theory section. To do this an arbitrary $6 \mathrm{~A}$-manipulator is needed, the $\mathrm{DH}-$ parameters of which are presented in Table 5.4.

Table 5.4: DH-parameters for 6A-manipulator inverse kinematics numeric example.

\begin{tabular}{|c||c|c|c|}
\hline link $i$ & $a_{i}$ & $a l_{i}$ & $d_{i}$ \\
\hline \hline 1 & 3 & $\frac{1}{3}$ & 0 \\
\hline 2 & 1 & 2 & $\frac{2}{3}$ \\
\hline 3 & $\frac{1}{2}$ & $\frac{3}{2}$ & 3 \\
\hline 4 & 4 & 3 & 2 \\
\hline 5 & $\frac{3}{4}$ & 1 & $\frac{1}{3}$ \\
\hline 6 & -2 & $\frac{1}{3}$ & 1 \\
\hline
\end{tabular}

\section{Obtaining the Target Pose}

Like in the 4A-manipulator numeric example of Section 5.1.2 a set of target joint variables is established prior to executing the algorithm to ensure the target EE pose is within the workspace of the manipulator. This set of joint variables is not used in the algorithm but should match one of the sets obtained in the end. In most cases these target joint variables would not be known and the results of the algorithm will indicate if the target pose is within 
the reachable workspace. The target set of joint variables is shown in Table 5.5. The target

Table 5.5: Target joint variables for 6A-manipulator inverse kinematics numeric example.

\begin{tabular}{|c||c|}
\hline joint $i$ & $u_{i}$ \\
\hline \hline 1 & $\frac{1}{2}$ \\
\hline 2 & -2 \\
\hline 3 & $\frac{3}{2}$ \\
\hline 4 & $\frac{1}{3}$ \\
\hline 5 & $-\frac{1}{3}$ \\
\hline 6 & 1 \\
\hline
\end{tabular}

pose is now obtained using the matrix equation

$$
\mathbf{T}_{E E}=\mathbf{M}_{1} \mathbf{G}_{1} \mathbf{M}_{2} \mathbf{G}_{2} \mathbf{M}_{3} \mathbf{G}_{3} \mathbf{M}_{4} \mathbf{G}_{4} \mathbf{M}_{5} \mathbf{G}_{5} \mathbf{M}_{6} \mathbf{G}_{6}
$$

which results in

$$
\mathbf{T}_{E E}=\left[\begin{array}{cccc}
1 & 0 & 0 & 0 \\
-\frac{492468563991}{85820312500} & \frac{583119847}{634765625} & \frac{192295088928}{536376953125} & -\frac{89071893096}{536376953125} \\
\frac{531782438834}{64365234375} & -\frac{123395904}{634765625} & \frac{23715226979}{536376953125} & -\frac{525609824028}{536376953125} \\
\frac{989287246}{2574609375} & -\frac{8733576}{25390625} & \frac{20006415876}{21455078125} & \frac{2366684293}{21455078125}
\end{array}\right]
$$

The inverse kinematics algorithm is now used to find the set(s) of joint angles that achieve this target EE pose.

\section{Left 3A-chain}

The 6A-manipulator is broken into two 3A-chains as described in Section 5.2.1. For each of these chains the constraint variety must be developed.

The left 3A-chains contains links and joints 1, 2 and 3 in that order. Starting with the set of equations in Equation (4.7) that represents the canonical constraint variety of a $2 \mathrm{~A}$ chain, the procedure for obtaining the constraint variety is described in Section 5.2.1. The 
resulting set of equations contains the Study Quadric and is a function of the eight Study parameters and the joint variable $u_{3}$. The intersection of these equations is the constraint variety of the left 3A-chain. The equations are shown in Appendix C.1.

\section{Right 3A-chain}

The right chain, with the new base located at the target $\mathrm{EE}$ pose of the $6 \mathrm{~A}$-manipulator contains the links and joints 6,5 and 4, in that order, going from the base of the right chain to $\Sigma_{R}$. The derivation of the constraint variety begins with the canonical 2A-chain constraint variety set of equations in Equation (4.7) and setting $a_{1}$ and $a l_{1}$ to $-a_{5}$ and $-a l_{5}$ respectively. To move to the new base at the EE pose of the $6 \mathrm{~A}$-manipulator the matrix $\mathrm{A}$ is found by Equation (5.2) using $\mathbf{G}_{6}^{-1}$ in place of $\mathbf{G}_{4}^{-1}$, the Study parameters of $\mathbf{A}$ are found in order to build $\mathbf{T}_{b}(\mathbf{A})$, and Equation (5.3) is used to obtain $\mathbf{x}^{\prime}$ which are substituted for $\mathbf{x}$ in the new set of equations. The joint offset of the second joint is accounted for using $\mathbf{G}_{5}^{\prime}$ which is found by Equation (5.4) with $-d_{5}$ substituted for $-d_{3}$. The procedure for transforming the set of equations describing the constraint variety is the same, using $\mathbf{T}_{m}\left(\mathbf{G}_{5}^{\prime}\right)$ in the moving frame. The third joint variable, $u_{4}$, is added using $\mathbf{T}_{m}\left(\mathbf{M}_{6}\right)$ and the regular procedure for modifying the set of equations. The equations are shown in Appendix C.2.

The intersection of the resulting set of equations represents the constraint variety of the right 3A-chain. The intersection lies on $S_{6}^{2}$ which is unchanged in the set of equations. The remaining four equations are functions of the eight homogenous Study parameters and the joint variable $u_{4}$.

\section{Intersecting the Constraint Varieties}

Obtaining the intersection of the left and right constraint varieties requires finding all sets of Study parameters and joint variables that satisfy both sets of five equations. Since both constraint varieties lie on $S_{6}^{2}$, the set of equations to be solved is reduced to nine equations in terms of the eight unknown homogenous Study Parameters and two joint variables. One of the eight Study parameters is set equal to unity because of homogeneity to give a system 
of nine equations in nine unknowns (seven Study parameters and two joint variables).

Attempting to solve the set of equations symbolically, similar to the $4 \mathrm{~A}$-manipulator method of resultant elimination, used in Section 5.1.2 produces equations too large to handle on a standard desktop computer. An Intel Pentium D, 3.00GHz, 1GB RAM with Maple 11 symbolic computer algebra software was used for this analysis. It is apparent that the system of equations is too large for the proposed symbolic algebra approach and thus it may be appropriate to apply a numerical approach.

Polynomial homotopy continuation, discussed in Section 2.11, appears to be an appropriate approach to solving this large system of polynomials because this method is designed to use path tracking from solutions of a known starting system that is carefully selected to satisfy the properties of triviality, smoothness and accessability (see Section 2.11.2) to find all solutions to the system of equations. To apply polynomial homotopy continuation methods to the problem of obtaining the intersections of the left and right 3A-chain constraint varieties a software package called PHCpack is used. This software package has been created and documented by Verschelde [27]. The input to the software package is the system of polynomials describing the left and right 3A-chain constraint varieties, the default software settings were used. PHCpack determined that the upper bound on the number of solutions should be 78944 and assembled the start system accordingly. The path tracking procedure was performed on a computer with an Intel Pentium D, 3.00GHz processor with 1GB RAM. This procedure took 6 days, 20 hours and 31 minutes to track all roots and resulted in 135 real solutions, 6703 complex solutions and 72106 paths that resulted in failure. It is important to note that if the path tracking procedure was assigned across multiple processors the time could be greatly reduced.

The number of paths that resulted in solutions, 135 real plus 6703 complex, is surprising because as discussed in Section 3.2.1 the solution to the inverse kinematics problem for a $6 \mathrm{R}$ manipulator possesses 16 solutions. It may be possible that the $6 \mathrm{~A}$-manipulator possesses more than 16 solutions to the inverse kinematics problem, however at this time these results must be viewed with reasonable skepticism. The path tracking procedure involves numerical methods which may introduce errors during its application or produce misleading results. 
Errors can occur because of such things as error bounds being too large or too small. Large error bounds may result in a solution that is not very close to correct being accepted as correct. Error bounds that are too small may result in two paths that converge on the same root being seen as separate roots by the software. Another issue with path tracking is when the solution jumps paths. If two or more solution paths are very close together at some value of $t$, it is possible that the tracking algorithm may inadvertently switch to the wrong path because of a large corrector step or too many corrections. This is why it is desirable to keep the number of corrections at each value of $t$ small. Even a jumped path that ends in a finite solution should result in a valid solution, but the solution set would then be incomplete.

It is important to note that the values of joint variables $u_{3}$ and $u_{4}$ of Table 5.5 were included in the set of real solutions. With this solution to the system it is possible to illustrate the rest of the algorithm. This was the only solution tested that yielded the target EE pose.

\section{Obtaining the Remaining Joint Variables}

For the joint variables $u_{3}$ and $u_{4}$ obtained using polynomial homotopy continuation that matched those of Table 5.5 the imaginary component of the solution is on the order of $10^{-33}$ and has therefore been excluded. The values obtained for this solution are $u_{3}=$ $1.500000000000166, u_{4}=0.333333333333411$ and the Study parameters of the coincident $\Sigma_{L}$ and $\Sigma_{R}$ are

$$
\left(\begin{array}{l}
x_{0} \\
x_{1} \\
x_{2} \\
x_{3} \\
y_{0} \\
y_{1} \\
y_{2} \\
y_{3}
\end{array}\right)=\left(\begin{array}{c}
1 \\
-0.325073563574486 \\
0.722936348149425 \\
0.135666718290128 \\
2.325612632682928 \\
1.626699110089408 \\
-2.686770072630170 \\
1.072834887996944
\end{array}\right)
$$


To solve for joint variables $u_{1}$ and $u_{2}$ the joint variable $u_{3}$ is substituted into Equation (5.11). The Study parameters of $\mathbf{E E}_{L}$ are obtained and set equal to and the Study parameters of Equation (5.15). After normalizing by dividing all equations by the first equation this results in a system of seven equations in the two unknown joint variables. Solving this over determined system in the same way as was done in Section 5.1.1 yields the values $u_{1}=0.500000000000004$ and $u_{2}=-1.99999999999997$ for the two joint variables. These results match the initial joint variables of Table 5.5 .

Obtaining the joint variables $u_{5}$ and $u_{6}$ requires the extra step of obtaining $\mathbf{E E}_{T-\text { relative }}$ by Equation (5.6) and obtaining the Study parameters. Equation (5.12) is used to obtain matrix $\mathbf{T}_{R}$ and its Study parameters which are functions of the joint variables $u_{5}$ and $u_{6}$. These functions are set equal to the Study parameters of $\mathbf{E E}_{T-\text { relative }}$ and after normalizing by dividing all equations by the first forms a system of seven equations in two unknowns. Solving this over determined system in the same manner as in section 5.1.1 yields the joint variables $u_{5}=-.333333333333293$ and $u_{6}=1.00000000000011$. These match the initial joint variables of Table 5.5. Table 5.6 shows the starting joint variables with the one set of joint variables obtained by this algorithm.

Table 5.6: Comparison of target joint variables with one set of the resulting joint variables for 6A-manipulator inverse kinematics numeric example.

\begin{tabular}{|c||c|c|}
\hline joint $i$ & Target & Algorithm \\
\hline \hline 1 & $\frac{1}{2}$ & 0.500000000000004 \\
\hline 2 & -2 & -1.99999999999997 \\
\hline 3 & $\frac{3}{2}$ & 1.500000000000166 \\
\hline 4 & $\frac{1}{3}$ & 0.333333333333411 \\
\hline 5 & $-\frac{1}{3}$ & -.333333333333293 \\
\hline 6 & 1 & 1.00000000000011 \\
\hline
\end{tabular}

These results match closely the initial values for the joint variables of Table 5.5 used to create the initial problem. This is however not the end of the inverse kinematics problem. The complete closed form solution must provide all of the sets of joint variables that place the EE in the desired pose. The large number of solutions provided by PHCpack made 
checking all the results impractical. Only a select few solutions were tested and of those only the solution where $u_{3}$ and $u_{4}$ matched the initial values of Table 5.5 did the resulting joint variables place the $\mathrm{EE}$ in the target pose. This indicates that the way the polynomial homotopy continuation method is applied must be re-evaluated. Manipulation of the software settings may result in better, more efficient solving of the intersection problem. This is an area that should be investigate during future research. 


\section{Chapter 6}

\section{Conclusions and Future Research}

This thesis has presented a novel architecture for a hybrid parallel-serial manipulator with the intent of improving the stiffness-to-weight ratio of serial R-manipulators. This is accomplished by introducing a new class of kinematic pair as the joints in a serial chain constructed from a special configuration of Griffis-Duffy platform subject to self-motions. Focus has been placed on the direct and inverse kinematics of this new A-chain architecture. The work presented does not bring complete closure to the topic and more research on kinematics, as well other aspects of the motion and construction, of the new hybrid parallel-serial manipulator constructed using A-pairs.

\subsection{Concluding Remarks}

The self-motions of a specific configuration of Griffis-Duffy parallel manipulator were employed as a novel kinematic pair, called an A-pair, with the characteristic that the rotation of the joint is coupled with a sinusoidal translation along the joint axis. The A-pair is used as a joint to connect links in a serial kinematic chain. The direct kinematic equations were obtained using DH-parameters and an algorithm for solving the inverse kinematics was proposed that parallels an algorithm for the inverse kinematics of a $6 \mathrm{R}$-manipulator using kinematic mapping.

The inverse kinematics algorithm has been presented for a $4 \mathrm{~A}$-manipulator. The basic theory involves splitting the manipulator into two $2 \mathrm{~A}$-chains and obtaining the constraint 
varieties for each sub-chain. The intersection of the constraint varieties is used to obtain the sets of joint variables that solve the inverse kinematics problem. A numerical example has been presented to illustrate the algorithm and in this case exactly one solution was obtained.

The algorithm was adapted for the inverse kinematics problem of a $6 \mathrm{~A}$-manipulator by splitting the manipulator into two 3A-chains. The constraint varieties of the $3 \mathrm{~A}$-chains are intersected to obtain the solutions. A numeric example has been presented but the prohibitive number of terms in the equations makes obtaining all intersection points difficult using both algebraic and numerical methods. One solution was obtained but further research is required to develop a procedure for determining the remainder of the solutions.

\subsection{Suggestions for Future Research}

The results of this thesis provide the analysis of kinematic chains using A-pairs for the first time. There are still possibilities for research in the area of kinematics as well as exploring other aspects of the novel architecture such as dynamics, control and force analysis. The following are some suggestions of research problems that should be addressed.

- Obtain the closed solution for the $6 \mathrm{~A}$-manipulator by completing the solution to the intersection problem presented in Section 5.2.2. This will require an examination of the equations representing the $3 \mathrm{~A}$-chain constraint varieties and the methods of solution. Intelligent manipulation of the settings on the PHPpack software may produce better results.

- The issue of the stiffness of A-pairs versus that of R-pairs must be explored to determine if the architecture does in fact produce a stiffer manipulator in practice.

- Explore other configurations of Griffis-Duffy platforms. The special configuration used in this thesis couples only rotation and translation. In other configurations the $\mathrm{DH}-$ parameter $\alpha_{i}$ would also be affected because the fixed base and moving platform would 
not necessarily remain parallel. This introduces more complexity into the equations but would produce interesting manipulator motions.

- In this thesis self-collisions in the A-pairs were ignored. An investigation of the joint limits should be conducted to determine the workspace limitations of the architecture.

- A method of controlling the A-pair is vital to the functionality of a manipulator constructed using A-pairs. An examination of how points on the moving platform of A-pair move relative to the fixed base may reveal a suitable method of controlling the joints.

- The static forces in the A-manipulator should be explored because, due to the coupling of rotation and translation in the A-pair, applying a force at the EE of the manipulator produces moments and linear forces at each joint.

- This thesis looks at static positions of A-manipulators. An examination of the motion and dynamics of the manipulators is required to develop control algorithms and to control the motion of the EE in space.

This list is by no means exhaustive but represents a good starting point for future research in the area of A-pairs and A-chains. 


\section{List of References}

[1] M. Zhao, T. Gui, G. Chao, Q. Li, and D. Tan, "Development of a redundant robot manipulator based on three DOF parallel platforms," in Proceedings of the 1995 IEEE International Conference on Robotics and Automation, (Nagoya, Japan), pp. 221-226, May 1995.

[2] M. Pfurner, Analysis of Spatial Serial Manipulators Using Kinematic Mapping. PhD thesis, University of Innsbruck, Austria, October 2006.

[3] J. Shigley and J. Uicker, Theory of Machines and Mechanisms. New York: McGrawHill, Inc., 1995.

[4] K. Brunnthaler, Synthesis of $4 R$ Linkages Using Kinematic Mapping. PhD thesis, University of Innsbruck, Austria, December 2006.

[5] K. Brunnthaler, H.-P. Schröcker, and M. Husty, "A new method for the synthesis of Bennett mechanisms," in Proceedings of CK2005, International Workshop on Computational Kinematics, pp. 93-113, September 1973.

[6] K. Hunt, Kinematic Geometry of Mechanisms. New York: Oxford University Press, 1990.

[7] J. Merlet, Parallel Manipulators. New York: Springer, 2006.

[8] G. Carbone and M. Ceccarelli, "A stiffness analysis for a hybrid parallel-serial manipulator," Robotica, vol. 22, pp. 567-576, 2004.

[9] M. Husty, "An algorithm for solving the direct kinematics of general Stewart-Gough platforms," Mechanism and Machine Theory, vol. 31, no. 4, pp. 365-380, 1996.

[10] X. Zheng, H. Bin, and Y. Luo, "Kinematic analysis of a hybrid serial-parallel manipulator," International Journal of Advanced Manufacturing Technology, vol. 23, pp. 925930, 2004.

[11] L. Romdhane, "Design and analysis of a hybrid serial-parallel manipulator," Machine and Mechanism Theory, vol. 34, pp. 1037-1055, 1999. 
[12] O. Ibrahim and W. Khalil, "Inverse dynamic modeling of serial-parallel hybrid robots," in Proceedings of the 2006 IEEE/RSJ International Intelligent Robots and Systems, (Beijing, China), pp. 2156-2161, October 2006.

[13] M. Hayes, P. Zsomber-Murray, and C. Chen, "Unified kinematic analysis of general planar parallel manipulators," ASME Journal of Mechanical Design, vol. 126, no. 5, pp. 866-874, 2004.

[14] J. Craig, Introduction to Robotics Mechanics and Control. Upper Saddle River, New Jersey: Pearson Prentice Hall, 2005.

[15] O. Bottema and B. Roth, Theoretical Kinematics. New York: North-Holland Publishing Company, 1979.

[16] W. Hamilton, "On quaternions," in Proceedings of the Royal Irish Academy, pp. 1-16, 1847.

[17] E. Study, Geometrie der Dynamen. Leipzig, Germany: B.G. Teubner, 1903.

[18] J. Denavit and R. Hartenberg, "A kinematic notation for lower-pair mechanisms based on matrices," Journal of Applied Mechanics, vol. 77, pp. 215-221, 1955.

[19] M. Husty, M. Pfurner, H.-P. Schröcker, and K. Brunnthaler, "Algebraic methods in mechanism analysis and synthesis," Robotica, vol. 25, pp. 661-675, 2007.

[20] D. Cox, J. Little, and D. O'Shea, Ideals, Varieties and Algorithms: An Introduction to Computational Algebraic Geometry and Commutative Algebra. New York: Springer, 1997.

[21] W. Adams and P. Loustaunau, An Introduction to Gröbner Bases. United States: American Mathematical Society, 1994.

[22] A. Sommese and C. Wampler, The Numerical Solution of Systems of Polynomials Arising in Engineering and Science. New Jersey: World Scientific, 2005.

[23] T. Li, "Numerical solution of multivariate polynomial systems by homotopy continuation methods," ACTA Numerica, vol. 6, pp. 399-436, 1997.

[24] M. Raghavan and B. Roth, "Computing all solutions to polynomial systems using homotopy continuation," AppliedMathematics and Computation, vol. 24, pp. 115-138, 1987.

[25] C. Wampler, A. Morgan, and A. Sommese, "Numerical continuation methods for solving polynomial systems arising in kinematics," Journal of Mechanical Design, vol. 112, pp. 59-68, 1990. 
[26] A. Morgan and A. Sommese, "Solving polynomial systems for the kinematic analysis and synthesis of mechanisms and robot manipulators," ASME Journal of Mechanical Design, vol. 126, pp. 71-79, 1995.

[27] J. Verschelde, "Algorithm 795: Phcpack: A general-purpose solver for polynomial systems by homotopy continuation," ACM Transactions on Mathematical Software, vol. 25 , no. 2 , pp. 251-276, 1999.

[28] M. Griffis and J. Duffy, "Method and apparatus for controlling geometrically simple parallel mechanisms with distinctive connections." U.S. Patent Number: 5,179,525, Jan 1993.

[29] M. Husty and A. Karger, "Self-motions of Griffis-Duffy type parallel manipulators," 2000 .

[30] A. Karger and M. Husty, "Classification of all self-motions of the original StewartGough platform," Computer-Aided Design, vol. 30, no. 3, pp. 2005-215, 2007.

[31] M. Husty and A. Karger, "Self motions of Stewart-Gough platforms, an overview," in Proceedings of the workshop on fundamental issues and future research directions for parallel mechanisms and manipulators, (Quebec City, Canada), pp. 131-141, October 2002.

[32] N. Chen and G. Parker, "Inverse kinematics solution to a calibrated PUMA 560 industrial robot," Control Engineering Practice, vol. 2, no. 2, pp. 239-245, 1994.

[33] J. Lloyd and V. Hayward, "Kinematics of common industrial robots," Robotics and Autonomous Systems, vol. 4, no. 2, pp. 169-191, 1988.

[34] R. Manseur and K. Doty, "Structural kinematics of 6-revolute-axis robot manipulators," Mechanism and Machine Theory, vol. 31, no. 5, pp. 647-657, 1996.

[35] R. Manseur and K. Doty, "Real-time inverse kinematics for robots with offset and reduced wrist," Control Engineering Practice, vol. 5, no. 10, pp. 1143-1450, 1997.

[36] F. Chapelle and P. Bidaud, "A closed form for inverse kinematics approximation of general 6R manipulators using genetic programming," in Proceedings of the 2001 IEEE International Conference on Robotics and Automation, (Seoul, Korea), pp. 3364-3369, May 2001.

[37] D. Pieper, The Kinematics of Manipulators Under Computer Control. PhD thesis, Stanford University, 1968.

[38] B. Roth, J. Rastegar, and V. Scheinmann, "On the design of computer controlled manipulators," in First CISM-IFToMM Symposium, (Cassino, Italy), May 2005. 
[39] J. Duffy and C. Crane, "A displacement analysis of the general spatial 7-link, 7R mechanism," Mechanism and Machine Theory, vol. 15, pp. 153-169, 1980.

[40] L. Tsai and A. Morgan, "Solving the kinematics of the most general six- and five-degreeof-freedom manipulators by continuation methods," Transactions of ASME, Journal of Mechanisms, Transmissions and Automation in Design, vol. 107, pp. 189-200, June 1985 .

[41] E. Primrose, "On the input-output equation of the general 7R-mechanism," Mechanism and Machine Theory, vol. 21, pp. 509-510, 1986.

[42] H. Lee and C. Liang, "Displacement analysis of the spatial 7-link 6R-P linkage," $M a-$ chine and Mechanism Theory, vol. 22, pp. 1-11, 1987.

[43] H. Lee and C. Liang, "Displacement analysis of the general 7-link 7R mechanism," Machine and Mechanism Theory, vol. 23, pp. 219-226, 1988.

[44] M. Raghavan and B. Roth, "Kinematic analysis of the $6 \mathrm{R}$ manipulator of general geometry," in Proceedings of the 5th International Symposium on Robotics Research, (Tokyo, Japan), 1990.

[45] D. Manocha and J. Canny, "Real time inverse kinematics for general 6R manipulators," in Proceedings of the IEEE International Conference on Robotics and Automation, (Nice, France), pp. 383-389, May 1992.

[46] D. Kohli and M. Osvatic, "Inverse kinematics of the general $6 \mathrm{R}$ and 5R,P serial manipulators," ASME Journal of Mechanical Design, vol. 115, pp. 922-931, 1993.

[47] M. Ghazvini, Computational Kinematics, ch. Reducing the Inverse Kinematics of Manipulators to the Solution of a Generalized Eigenproblem, pp. 15-26. Kluwer Academic Publisher, 1993.

[48] M. Husty, M. Pfurner, and H.-P. Schröcker, "A new and efficient algorithm for the inverse kinematics of a general serial 6R manipulator," Mechanism and Machine Theory, vol. 42 , no. 1 , pp. $66-81,2007$. 


\section{Appendix A}

\section{Equations of Constraint Variety for a Canonical}

\section{A-chain in Terms of $u_{i+1}$}

The equations of the constraint variety for a canonical 2A-chain in terms of joint variable $u_{i+1}$ are presented. Note that because of the size of some of the equations in this section they are presented in the format in which they are input into the Maple algebraic mathematics software. The equations are presented without subscripts and with exponents represented by the character "^, and the following number (i.e. $\mathrm{x} 1^{\wedge} 2$ is equivalent to $x_{1}^{2}$ ). Also note that $u_{i+1}$ is replaced with ' $\mathrm{u}$ ', $a l_{i}$ with 'al', $a_{i}$ with 'a' and $d_{i}$ with 'd'. The fonts used may also vary from those in the text of this thesis.

EQ1: $\mathrm{x} 0^{*} \mathrm{y} 0+\mathrm{x} 1^{*} \mathrm{y} 1+\mathrm{x} 2{ }^{*} \mathrm{y} 2+\mathrm{x} 3^{*} \mathrm{y} 3=0$

EQ2: $2^{*} \mathrm{x} 2^{*} \mathrm{u}+\left(-\mathrm{al}^{*} \mathrm{u}^{\wedge} 2+\mathrm{al}\right)^{*} \mathrm{x} 0+\mathrm{x} 1^{*}\left(-1+\mathrm{u}^{\wedge} 2\right)+2{ }^{*} \mathrm{x} 3^{*} \mathrm{u}^{*} \mathrm{al}=0$

EQ3: $\mathrm{x} 2^{*}\left(-1+\mathrm{u}^{\wedge} 2\right)-2^{*} \mathrm{x} 0^{*} \mathrm{u}^{*} \mathrm{al}-2^{*} \mathrm{x} 1^{*} \mathrm{u}+\left(-\mathrm{al}^{*} \mathrm{u}^{\wedge} 2+\mathrm{al}\right)^{*} \mathrm{x} 3=0$

EQ4: $\left(2^{*} \mathrm{al}-2^{*} \mathrm{al}^{*} \mathrm{u}^{\wedge} 4\right)^{*} \mathrm{y} 0+\left(4^{*} \mathrm{u}+4^{*} \mathrm{u}^{\wedge} 3\right)^{*} \mathrm{y} 2+\left(2^{*} \mathrm{u}^{\wedge} 3^{*} \mathrm{a} \mathrm{l}^{*} \mathrm{a}+2^{*} \mathrm{u}^{\wedge} 3-2^{*} \mathrm{u}+\right.$ $\left.2^{*} u^{*} a l^{* a}\right)^{*} \mathrm{x} 2+\left(-2+2^{*} u^{\wedge} 4\right)^{*} \mathrm{y} 1+\mathrm{x} 0^{*}\left(\mathrm{a}^{*} \mathrm{u}^{\wedge} 4+4^{*} \mathrm{al}^{*} \mathrm{u}^{\wedge} 2-\mathrm{a}\right)+\mathrm{x} 1^{*}\left(\mathrm{al} \mathbf{l}^{*} \mathrm{a}^{*} \mathrm{u}^{\wedge} 4-4^{*} \mathrm{u}^{\wedge} 2-\right.$ $\left.\left.\mathrm{al}^{*} \mathrm{a}\right)+\left(4^{*} \mathrm{u}^{\wedge} 3^{*} \mathrm{al}+4^{*} \mathrm{u}^{*} \mathrm{al}\right)\right)^{*} \mathrm{y} 3+\left(-2^{*} \mathrm{u}^{*} \mathrm{al}-2^{*} \mathrm{u}^{*} \mathrm{a}+2^{*} \mathrm{u}^{\wedge} 3^{*} \mathrm{al}-2^{*} \mathrm{u}^{\wedge} 3^{*} \mathrm{a}\right)^{*} \mathrm{x} 3=0$

EQ5: $\left.\left(-4^{*} \mathrm{u}^{*} \mathrm{al}-4^{*} \mathrm{u}^{\wedge} 3^{*} \mathrm{al}\right)\right)^{*} \mathrm{y} 0+\left(-2+2^{*} \mathrm{u}^{\wedge} 4\right)^{*} \mathrm{y} 2+\mathrm{x} 2^{*}\left(\mathrm{al}^{*} \mathrm{a}^{*} \mathrm{u}^{\wedge} 4-4^{*} \mathrm{u}^{\wedge} 2-\mathrm{al}^{*} \mathrm{a}\right)+(-$ $\left.4^{*} \mathrm{u}-4^{*} \mathrm{u}^{\wedge} 3\right)^{*} \mathrm{y} 1+\left(2^{*} \mathrm{u}^{\wedge} 3^{*} \mathrm{a}+2^{*} \mathrm{u}^{*} \mathrm{al}+2^{*} \mathrm{u}^{*} \mathrm{a}-2^{*} \mathrm{u}^{\wedge} 3^{*} \mathrm{al}\right)^{*} \mathrm{x} 0+\left(-2^{*} \mathrm{u}^{\wedge} 3+2^{*} \mathrm{u}-\right.$ $\left.2^{*} u^{*} a l^{*} a-2^{*} u^{\wedge} 3^{*} a l^{*} a\right)^{*} x 1+\left(2^{*} a l-2^{*} a l^{*} u^{\wedge} 4\right)^{*} y 3+x 3^{*}\left(a^{*} u^{\wedge} 4+4^{*} a l^{*} u^{\wedge} 2-\right.$ a $)=0$

EQ6: $\left(-24^{*} \mathrm{u}^{\wedge} 4-4-16^{*} \mathrm{u}^{\wedge} 2-4^{*} \mathrm{u}^{\wedge} 8-16^{*} \mathrm{u}^{\wedge} 6\right)^{*} \mathrm{y} 0^{\wedge} 2+\left(\left(-32^{*} \mathrm{u}^{\wedge} 5^{*} \mathrm{al}-32^{*} \mathrm{u}^{\wedge} 7^{*} \mathrm{al}+\right.\right.$ $\left.32^{*} \mathrm{u}^{*} \mathrm{al}+32^{*} \mathrm{u}^{\wedge} 3^{*} \mathrm{al}\right)^{*} \mathrm{y} 2+\left(-16^{*} \mathrm{u}^{\wedge} 3^{*} \mathrm{a}+16^{*} \mathrm{u}^{\wedge} 5^{*} \mathrm{a}+40^{*} \mathrm{u}^{\wedge} 3^{*} \mathrm{al}+40^{*} \mathrm{u}^{\wedge} 5^{*} \mathrm{al}+\right.$ $\left.16^{*} \mathrm{u}^{\wedge} 7^{*} \mathrm{a}-8^{*} \mathrm{u}^{*} \mathrm{al}-16^{*} \mathrm{u}^{*} \mathrm{a}-8^{*} \mathrm{u}^{\wedge} 7^{*} \mathrm{al}\right)^{*} \mathrm{x} 2+\left(-8^{*} \mathrm{al}+32^{*} \mathrm{al} \mathbf{l}^{*} \mathrm{u}^{\wedge} 2+32^{*} \mathrm{al}{ }^{*} \mathrm{u}^{\wedge} 6-8^{*} \mathrm{al} \mathrm{l}^{*} \mathrm{u}^{\wedge} 8\right.$ $\left.+80^{*} a l^{*} u^{\wedge} 4\right)^{*} y 1+\left(-16^{*} a l^{*} a^{*} u^{\wedge} 6-16^{*} a l^{*} a^{*} u^{\wedge} 2-24^{*} a l^{*} a^{*} u^{\wedge} 4-4^{*} a l^{*} a-4^{*} a l^{*} a^{*} u^{\wedge} 8\right)^{*} x 0$ $+\left(-16^{*} a^{*} u^{\wedge} 2-40^{*} a^{*} u^{\wedge} 4-32^{*} a l^{*} u^{\wedge} 2+32^{*} a l^{*} u^{\wedge} 6+4^{*} a^{*} u^{\wedge} 8-16^{*} a^{*} u^{\wedge} 6+4^{*} a\right)^{*} x 1+$ 
$\left.\left(8^{*} \mathrm{u}+24^{*} \mathrm{u}^{\wedge} 3+24^{*} \mathrm{u}^{\wedge} 5+8^{*} \mathrm{u}^{\wedge} 7\right)^{*} \mathrm{x} 3\right)^{*} \mathrm{y} 0+\left(-24^{*} \mathrm{al}^{\wedge} 2^{*} \mathrm{u}^{\wedge} 4-16^{*} \mathrm{u}^{\wedge} 2^{*} \mathrm{al}^{\wedge} 2-4^{*} \mathrm{al}^{\wedge} 2-\right.$ $\left.16^{*} \mathrm{u}^{\wedge} 6^{*} \mathrm{al}^{\wedge} 2-4^{*} \mathrm{u}^{\wedge} 8^{*} \mathrm{a} l^{\wedge} 2\right)^{*} \mathrm{y} 2^{\wedge} 2+\left(\left(16^{*} \mathrm{a} l^{*} \mathrm{a}^{*} \mathrm{u}^{\wedge} 6+16^{*} \mathrm{a}{ }^{*} \mathrm{a}^{*} \mathrm{u}^{\wedge} 2+24^{*} \mathrm{al}{ }^{*} \mathrm{a}^{*} \mathrm{u}^{\wedge} 4+\right.\right.$ $\left.4^{*} \mathrm{al}^{*} \mathrm{a}^{*} \mathrm{u}^{\wedge} 8+4^{*} \mathrm{al}{ }^{*} \mathrm{a}\right)^{*} \mathrm{x} 2+\left(40^{*} \mathrm{u}^{\wedge} 3^{*} \mathrm{al}+40^{*} \mathrm{u}^{\wedge} 5^{*} \mathrm{al}-8^{*} \mathrm{u}^{\wedge} 7^{*} \mathrm{al}+16^{*} \mathrm{u}^{*} \mathrm{al}^{\wedge} 2^{*} \mathrm{a}+\right.$ $\left.16^{*} \mathrm{u}^{\wedge} 3^{*} \mathrm{al}^{\wedge} 2^{*} \mathrm{a}-16^{*} \mathrm{u}^{\wedge} 5^{*} \mathrm{a} \mathrm{l}^{\wedge} 2^{*} \mathrm{a}-8^{*} \mathrm{u}^{*} \mathrm{al}-16^{*} \mathrm{u}^{\wedge} 7^{*} \mathrm{al} \mathrm{l}^{\wedge} 2^{*} \mathrm{a}\right)^{*} \mathrm{x} 0+\left(8^{*} \mathrm{u}^{\wedge} 7^{*} \mathrm{al}^{\wedge} 2+8^{*} \mathrm{al}^{\wedge} 2^{*} \mathrm{u}+\right.$ $\left.24^{*} \mathrm{al}^{\wedge} 2^{*} \mathrm{u}^{\wedge} 3+24^{*} \mathrm{al}^{\wedge} 2^{*} \mathrm{u}^{\wedge} 5\right)^{*} \mathrm{x} 1+\left(-8^{*} \mathrm{al}+32^{*} \mathrm{al}^{*} \mathrm{u}^{\wedge} 2+32^{*} \mathrm{al}^{*} \mathrm{u}^{\wedge} 6-8^{*} \mathrm{al}^{*} \mathrm{u}^{\wedge} 8+\right.$ $\left.80^{*} \mathrm{al}^{*} \mathrm{u}^{\wedge} 4\right)^{*} \mathrm{y} 3+\left(-32^{*} \mathrm{al}^{*} \mathrm{u}^{\wedge} 2+16^{*} \mathrm{a}^{*} \mathrm{u}^{\wedge} 6^{*} \mathrm{al}^{\wedge} 2+16^{*} \mathrm{a}^{*} \mathrm{u}^{\wedge} 2^{*} \mathrm{al}^{\wedge} 2-4^{*} \mathrm{al}^{\wedge} 2^{*} \mathrm{a}+\right.$ $\left.\left.40^{*} \mathrm{al}^{\wedge} 2^{*} \mathrm{a}^{*} \mathrm{u}^{\wedge} 4-4^{*} \mathrm{a}^{*} \mathrm{u}^{\wedge} 8^{*} \mathrm{al}^{\wedge} 2+32^{*} \mathrm{al}^{*} \mathrm{u}^{\wedge} 6\right)^{*} \mathrm{x} 3\right)^{*} \mathrm{y} 2+\left(\mathrm{al}^{\wedge} 2+\mathrm{u}^{\wedge} 8^{*} \mathrm{a} l^{\wedge} 2-4^{*} \mathrm{u}^{\wedge} 6^{*} \mathrm{al}^{\wedge} 2-\right.$ $\left.a^{\wedge} 2^{*} u^{\wedge} 8-4^{*} a^{\wedge} 2^{*} u^{\wedge} 2-a^{\wedge} 2-6^{*} a^{\wedge} 2^{*} u^{\wedge} 4-4^{*} a^{\wedge} 2^{*} u^{\wedge} 6-10^{*} a l^{\wedge} 2^{*} u^{\wedge} 4-4^{*} u^{\wedge} 2^{*} a l^{\wedge} 2\right)^{*} x 2^{\wedge} 2+$ $\left(\left(-24^{*} \mathrm{al}^{\wedge} 2^{*} \mathrm{u}^{\wedge} 3-8^{*} \mathrm{a} \mathrm{l}^{\wedge} 2^{*} \mathrm{u}-24^{*} \mathrm{al}^{\wedge} 2^{*} \mathrm{u}^{\wedge} 5-8^{*} \mathrm{u}^{\wedge} 7^{*} \mathrm{al}{ }^{\wedge} 2\right)^{*} \mathrm{y} 1+\left(-36^{*} \mathrm{u}^{\wedge} 3^{*} \mathrm{al}-4^{*} \mathrm{u}^{*} \mathrm{al}+\right.\right.$ $4^{*} \mathrm{u}^{\wedge} 7^{*} \mathrm{al}-20^{*} \mathrm{u}^{\wedge} 5^{*} \mathrm{a}+20^{*} \mathrm{u}^{\wedge} 3^{*} \mathrm{al}^{\wedge} 2^{*} \mathrm{a}+20^{*} \mathrm{u}^{\wedge} 5^{*} \mathrm{a}{ }^{\wedge} 2^{*} \mathrm{a}-8^{*} \mathrm{u}^{*} \mathrm{a} \mathbf{l}^{*} \mathrm{a}^{\wedge} 2-4^{*} \mathrm{u}^{\wedge} 7^{*} \mathrm{al}{ }^{\wedge} 2^{*} \mathrm{a}+$ $8^{*} \mathrm{u}^{\wedge} 5^{*} \mathrm{a}^{\wedge} 2^{*} \mathrm{al}-8^{*} \mathrm{u}^{\wedge} 3^{*} \mathrm{a} \mathbf{l}^{*} \mathrm{a}^{\wedge} 2-4^{*} \mathrm{u}^{*} \mathrm{al}^{\wedge} 2^{*} \mathrm{a}+8^{*} \mathrm{u}^{\wedge} 7^{*} \mathrm{al} \mathbf{l}^{*} \mathrm{a}^{\wedge} 2+36^{*} \mathrm{u}^{\wedge} 5^{*} \mathrm{al}+4^{*} \mathrm{u}^{\wedge} 7^{*} \mathrm{a}+$ $\left.4^{*} \mathrm{u}^{*} \mathrm{a}-20^{*} \mathrm{u}^{\wedge} 3^{*} \mathrm{a}\right)^{*} \mathrm{x} 0+\left(4^{*} \mathrm{al}^{\wedge} 2^{*} \mathrm{u}^{\wedge} 3-4^{*} \mathrm{u}^{\wedge} 7^{*} \mathrm{al} \mathrm{l}^{\wedge} 2+4^{*} \mathrm{al}^{\wedge} 2^{*} \mathrm{u}-4^{*} \mathrm{a} \mathrm{l}^{\wedge} 2^{*} \mathrm{u}^{\wedge} 5\right)^{*} \mathrm{x} 1+(-$ $\left.16^{*} a^{*} u^{\wedge} 2-40^{*} a^{*} u^{\wedge} 4-32^{*} a l^{*} u^{\wedge} 2+32^{*} a l^{*} u^{\wedge} 6+4^{*} a^{*} u^{\wedge} 8-16^{*} a^{*} u^{\wedge} 6+4^{*} a\right)^{*} y 3+$ $\left(16^{*} a^{*} u^{\wedge} 6^{*} a l^{\wedge} 2-16^{*} a^{*} u^{\wedge} 2^{*} a^{\wedge} 2+16^{*} a^{*} u^{\wedge} 2+8^{*} a l^{*} u^{\wedge} 2+2^{*} a l^{*} u^{\wedge} 8+2^{*} a^{\wedge} 2^{*} u^{\wedge} 8^{*} a l-\right.$ $20^{*} a^{\wedge} 2^{*} \mathrm{u}^{\wedge} 4^{*} \mathrm{al}-8^{*} \mathrm{a}^{\wedge} 2^{*} \mathrm{u}^{\wedge} 6^{*} \mathrm{al}-8^{*} \mathrm{al}^{*} \mathrm{a}^{\wedge} 2^{*} \mathrm{u}^{\wedge} 2+2^{*} \mathrm{al}^{*} \mathrm{a}^{\wedge} 2+8^{*} \mathrm{al}^{*} \mathrm{u}^{\wedge} 6-16^{*} \mathrm{a}^{*} \mathrm{u}^{\wedge} 6+2^{*} \mathrm{al}-$ $\left.\left.52^{*} \mathrm{al}^{*} \mathrm{u}^{\wedge} 4\right)^{*} \mathrm{x} 3\right)^{*} \mathrm{x} 2+\left(-24^{*} \mathrm{al}^{\wedge} 2^{*} \mathrm{u}^{\wedge} 4-16^{*} \mathrm{u}^{\wedge} 2^{*} \mathrm{al}^{\wedge} 2-4^{*} \mathrm{al}^{\wedge} 2-16^{*} \mathrm{u}^{\wedge} 6^{*} \mathrm{al}^{\wedge} 2-\right.$

$\left.4^{*} \mathrm{u}^{\wedge} 8^{*} \mathrm{al}^{\wedge} 2\right)^{*} \mathrm{y} 1^{\wedge} 2+\left(\left(-32^{*} \mathrm{al}^{*} \mathrm{u}^{\wedge} 2+16^{*} \mathrm{a}^{*} \mathrm{u}^{\wedge} 6^{*} \mathrm{al} \mathrm{l}^{\wedge} 2+16^{*} \mathrm{a}^{*} \mathrm{u}^{\wedge} 2^{*} \mathrm{a} \mathrm{l}^{\wedge} 2-4^{*} \mathrm{a} \mathrm{l}^{\wedge} 2^{*} \mathrm{a}+\right.\right.$ $\left.40^{*} \mathrm{al}^{\wedge} 2^{*} \mathrm{a}^{*} \mathrm{u}^{\wedge} 4-4^{*} \mathrm{a}^{*} \mathrm{u}^{\wedge} 8^{*} \mathrm{al}^{\wedge} 2+32^{*} \mathrm{al}^{*} \mathrm{u}^{\wedge} 6\right)^{*} \mathrm{x} 0+\left(16^{*} \mathrm{al}^{*} \mathrm{a}^{*} \mathrm{u}^{\wedge} 6+16^{*} \mathrm{al}^{*} \mathrm{a}^{*} \mathrm{u}^{\wedge} 2+\right.$ $\left.24^{*} \mathrm{al}^{*} \mathrm{a}^{*} \mathrm{u}^{\wedge} 4+4^{*} \mathrm{a} \mathbf{l}^{*} \mathrm{a}^{*} \mathrm{u}^{\wedge} 8+4^{*} \mathrm{al}^{*} \mathrm{a}\right)^{*} \mathrm{x} 1+\left(-32^{*} \mathrm{u}^{*} \mathrm{al}+32^{*} \mathrm{u}^{\wedge} 5^{*} \mathrm{al}+32^{*} \mathrm{u}^{\wedge} 7^{*} \mathrm{al}-\right.$ $\left.32^{*} \mathrm{u}^{\wedge} 3^{*} \mathrm{al}\right)^{*} \mathrm{y} 3+\left(-16^{*} \mathrm{u}^{*} \mathrm{al} \mathrm{l}^{\wedge} 2^{*} \mathrm{a}+8^{*} \mathrm{u}^{*} \mathrm{al}-40^{*} \mathrm{u}^{\wedge} 3^{*} \mathrm{al}-16^{*} \mathrm{u}^{\wedge} 3^{*} \mathrm{al}^{\wedge} 2^{*} \mathrm{a}+8^{*} \mathrm{u}^{\wedge} 7^{*} \mathrm{al}+\right.$ $\left.\left.16^{*} \mathrm{u}^{\wedge} 7^{*} \mathrm{al}^{\wedge} 2^{*} \mathrm{a}-40^{*} \mathrm{u}^{\wedge} 5^{*} \mathrm{al}+16^{*} \mathrm{u}^{\wedge} 5^{*} \mathrm{al}^{\wedge} 2^{*} \mathrm{a}\right)^{*} \mathrm{x} 3\right)^{*} \mathrm{y} 1+\left(-4^{*} \mathrm{al}^{\wedge} 2^{*} \mathrm{a}^{\wedge} 2^{*} \mathrm{u}^{\wedge} 2-\right.$

$\left.6^{*} \mathrm{al}^{\wedge} 2^{*} \mathrm{a}^{\wedge} 2^{*} \mathrm{u}^{\wedge} 4-4^{*} \mathrm{al}^{\wedge} 2^{*} \mathrm{a}^{\wedge} 2^{*} \mathrm{u}^{\wedge} 6-\mathrm{al}^{\wedge} 2^{*} \mathrm{a}^{\wedge} 2-\mathrm{al}^{\wedge} 2^{*} \mathrm{a}^{\wedge} 2^{*} \mathrm{u}^{\wedge} 8\right)^{*} \mathrm{x} 0^{\wedge} 2+\left(\left(2^{*} \mathrm{a} \mathrm{l}^{*} \mathrm{a}^{\wedge} 2-\right.\right.$

$16^{*} \mathrm{a}^{*} \mathrm{u}^{\wedge} 6+2^{*} \mathrm{a}^{\wedge} 2^{*} \mathrm{u}^{\wedge} 8^{*} \mathrm{al}-20^{*} \mathrm{a}^{\wedge} 2^{*} \mathrm{u}^{\wedge} 4^{*} \mathrm{al}-8^{*} \mathrm{al}^{*} \mathrm{a}^{\wedge} 2^{*} \mathrm{u}^{\wedge} 2+16^{*} \mathrm{a}^{*} \mathrm{u}^{\wedge} 6^{*} \mathrm{al} \mathrm{al}^{\wedge} 2+16^{*} \mathrm{a}^{*} \mathrm{u}^{\wedge} 2$ $\left.8^{*} \mathrm{a}^{\wedge} 2^{*} \mathrm{u}^{\wedge} 6^{*} \mathrm{al}-16^{*} \mathrm{a}^{*} \mathrm{u}^{\wedge} 2^{*} \mathrm{al}^{\wedge} 2-64^{*} \mathrm{al}^{*} \mathrm{u}^{\wedge} 4\right)^{*} \mathrm{x} 1+\left(-8^{*} \mathrm{u}-24^{*} \mathrm{u}^{\wedge} 5-8^{*} \mathrm{u}^{\wedge} 7-24^{*} \mathrm{u}^{\wedge} 3\right)^{*} \mathrm{y} 3+$ $\left.\left(-4^{*} u^{\wedge} 3-4^{*} u+4^{*} u^{\wedge} 5+4^{*} u^{\wedge} 7\right)^{*} x 3\right)^{*} x 0+\left(-6^{*} a^{\wedge} 2^{*} u^{\wedge} 4-a^{\wedge} 2-a^{\wedge} 2^{*} u^{\wedge} 8-4^{*} a^{\wedge} 2^{*} u^{\wedge} 2-\right.$ $\left.4^{*} \mathrm{a}^{\wedge} 2^{*} \mathrm{u}^{\wedge} 6\right)^{*} \mathrm{x} 1^{\wedge} 2+\left(\left(-16^{*} \mathrm{u}^{\wedge} 7^{*} \mathrm{a}+8^{*} \mathrm{u}^{\wedge} 7^{*} \mathrm{al}+16^{*} \mathrm{u}^{\wedge} 3^{*} \mathrm{a}-40^{*} \mathrm{u}^{\wedge} 3^{*} \mathrm{al}+8^{*} \mathrm{u}^{*} \mathrm{al}+16^{*} \mathrm{u}^{*} \mathrm{a}\right.\right.$ $\left.-16^{*} \mathrm{u}^{\wedge} 5^{*} \mathrm{a}-40^{*} \mathrm{u}^{\wedge} 5^{*} \mathrm{al}\right)^{*} \mathrm{y} 3+\left(4^{*} \mathrm{u}^{*} \mathrm{a} \mathrm{l}^{\wedge} 2^{*} \mathrm{a}-8^{*} \mathrm{u}^{\wedge} 7^{*} \mathrm{a} \mathbf{l}^{*} \mathrm{a}^{\wedge} 2-4^{*} \mathrm{u}^{\wedge} 7^{*} \mathrm{al}+20^{*} \mathrm{u}^{\wedge} 3^{*} \mathrm{a}+\right.$ $20^{*} u^{\wedge} 5^{*} \mathrm{a}+4^{*} \mathrm{u}^{\wedge} 7^{*} \mathrm{al} \mathbf{l}^{\wedge} 2^{*} \mathrm{a}-20^{*} \mathrm{u}^{\wedge} 5^{*} \mathrm{al} \mathrm{l}^{\wedge} 2^{*} \mathrm{a}-4^{*} \mathrm{u}^{\wedge} 7^{*} \mathrm{a}+36^{*} \mathrm{u}^{\wedge} 3^{*} \mathrm{al}+8^{*} \mathrm{u}^{*} \mathrm{al} \mathrm{l}^{*} \mathrm{a}^{\wedge} 2+4^{*} \mathrm{u}^{*} \mathrm{al}$ $\left.\left.-8^{*} \mathrm{u}^{\wedge} 5^{*} \mathrm{a}^{\wedge} 2^{*} \mathrm{al}+8^{*} \mathrm{u}^{\wedge} 3^{*} \mathrm{al}{ }^{*} \mathrm{a}^{\wedge} 2-20^{*} \mathrm{u}^{\wedge} 3^{*} \mathrm{a}{ }^{\wedge} 2^{*} \mathrm{a}-36^{*} \mathrm{u}^{\wedge} 5^{*} \mathrm{al}-4^{*} \mathrm{u}^{*} \mathrm{a}\right)^{*} \mathrm{x} 3\right)^{*} \mathrm{x} 1+\left(-24^{*} \mathrm{u}^{\wedge} 4\right.$ $\left.-4-16^{*} \mathrm{u}^{\wedge} 2-4^{*} \mathrm{u}^{\wedge} 8-16^{*} \mathrm{u}^{\wedge} 6\right)^{*} \mathrm{y} 3^{\wedge} 2+\left(-16^{*} \mathrm{a}\right)^{*} \mathrm{a}^{*} \mathrm{u}^{\wedge} 6-16^{*} \mathrm{al}^{*} \mathrm{a}^{*} \mathrm{u}^{\wedge} 2-24^{*} \mathrm{a} \mathrm{l}^{*} \mathrm{a}^{*} \mathrm{u}^{\wedge} 4-$ $\left.4^{*} \mathrm{a} l^{*} \mathrm{a}-4^{*} \mathrm{al}^{*} \mathrm{a}^{*} \mathrm{u}^{\wedge} 8\right)^{*} \mathrm{x} 3^{*} \mathrm{y} 3+\left(-\mathrm{al}^{\wedge} 2^{*} \mathrm{a}^{\wedge} 2^{*} \mathrm{u}^{\wedge} 8-4^{*} \mathrm{u}^{\wedge} 6-6^{*} \mathrm{al}^{\wedge} 2^{*} \mathrm{a}^{\wedge} 2^{*} \mathrm{u}^{\wedge} 4-\right.$ $\left.4^{*} \mathrm{al}^{\wedge} 2^{*} \mathrm{a}^{\wedge} 2^{*} \mathrm{u}^{\wedge} 6+1-4^{*} \mathrm{al}^{\wedge} 2^{*} \mathrm{a}^{\wedge} 2^{*} \mathrm{u}^{\wedge} 2-4^{*} \mathrm{u}^{\wedge} 2+\mathrm{u}^{\wedge} 8-10^{*} \mathrm{u}^{\wedge} 4-\mathrm{al}^{\wedge} 2^{*} \mathrm{a}^{\wedge} 2\right)^{*} \mathrm{x} 3^{\wedge} 2=0$ 


\section{Appendix B}

\section{Equations for 4A-manipulator Numeric Example}

In this Appendix equations for the numeric 4A-manipulator inverse kinematics example are presented. Note that because of the size of some of the equations in this section they are presented in the format in which they are input into the Maple algebraic mathematics software. The equations are presented without subscripts and with exponents represented by the character "^, and the following number (i.e. $\mathrm{x} 1^{\wedge} 2$ is equivalent to $x_{1}^{2}$ ). The fonts used may also vary from those in the text of this thesis.

\section{B.1 Left 2A-chain Constraint Variety}

The five Equations of the left constraint variety are as follows. The equations as presented are all set equal to zero. See Section 5.1.2.

$$
\begin{aligned}
& \mathrm{L} 1:=\mathrm{x} 0^{*} \mathrm{y} 0+\mathrm{x} 1^{*} \mathrm{y} 1+\mathrm{x} 2^{*} \mathrm{y} 2+\mathrm{x} 3^{*} \mathrm{x} 8 \\
& \mathrm{~L} 2:=275^{*} \mathrm{x} 2^{\wedge} 2+50^{*} \mathrm{x} 2^{*} \mathrm{y} 2+850^{*} \mathrm{x} 3^{\wedge} 2+850^{*} \mathrm{x} 0^{\wedge} 2-975^{*} \mathrm{x} 0^{*} \mathrm{x} 1+275^{*} \mathrm{x} 1^{\wedge} 2+200^{*} \mathrm{x} 0^{*} \mathrm{y} 0 \\
& -100^{*} \mathrm{y} 0^{*} \mathrm{x} 1-100^{*} \mathrm{y} 1^{*} \mathrm{x} 0-975^{*} \mathrm{x} 3^{*} \mathrm{x} 2-100^{*} \mathrm{y} 2^{*} \mathrm{x} 3-100^{*} \mathrm{x} 8^{*} \mathrm{x} 2+200^{*} \mathrm{x} 3^{*} \mathrm{x} 8+50^{*} \mathrm{x} 1^{*} \mathrm{y} 1 \\
& \mathrm{~L} 3:=-35^{*} \mathrm{x} 0^{\wedge} 2+40^{*} \mathrm{x} 0^{*} \mathrm{x} 1-5^{*} \mathrm{x} 1^{\wedge} 2-5^{*} \mathrm{x} 2^{\wedge} 2+40^{*} \mathrm{x} 3^{*} \mathrm{x} 2-35^{*} \mathrm{x} 3^{\wedge} 2 \\
& \mathrm{~L} 4:=2088^{*} \mathrm{x} 2^{*} \mathrm{y} 2-2556^{*} \mathrm{y} 2^{*} \mathrm{x} 3-2556^{*} \mathrm{y} 1^{*} \mathrm{x} 0-2270^{*} \mathrm{x} 0^{*} \mathrm{x} 1-2108^{*} \mathrm{x} 3^{*} \mathrm{x} 2-4356^{*} \mathrm{y} 0^{*} \mathrm{x} 1- \\
& 1247^{*} \mathrm{x} 3^{\wedge} 2-1355^{*} \mathrm{x} 0^{\wedge} 2-4356^{*} \mathrm{x} 8^{*} \mathrm{x} 2+1332^{*} \mathrm{y} 0^{\wedge} 2+468^{*} \mathrm{y} 1^{\wedge} 2+1332^{*} \mathrm{x} 8^{\wedge} 2-1152^{*} \mathrm{y} 0^{*} \mathrm{y} 1 \\
& -1152^{*} \mathrm{y} 2^{*} \mathrm{x} 8+120^{*} \mathrm{x} 2^{*} \mathrm{y} 1-840^{*} \mathrm{x} 0^{*} \mathrm{x} 8-480^{*} \mathrm{x} 3^{*} \mathrm{y} 1-480^{*} \mathrm{x} 2^{*} \mathrm{y} 0+2005^{*} \mathrm{x} 1^{\wedge} 2+ \\
& 1897^{*} \mathrm{x} 2^{\wedge} 2-480^{*} \mathrm{x} 1^{*} \mathrm{x} 3+5472^{*} \mathrm{x} 0^{*} \mathrm{y} 0+2088^{*} \mathrm{x} 1^{*} \mathrm{y} 1+5472^{*} \mathrm{x} 3^{*} \mathrm{x} 8+468^{*} \mathrm{y} 2^{\wedge} 2+ \\
& 480^{*} \mathrm{x} 1^{*} \mathrm{x} 8+480^{*} \mathrm{x} 0^{*} \mathrm{y} 2-120^{*} \mathrm{x} 1^{*} \mathrm{y} 2+840^{*} \mathrm{x} 3^{*} \mathrm{y} 0+480^{*} \mathrm{x} 0^{*} \mathrm{x} 2 \\
& \mathrm{~L} 5:=-540^{*} \mathrm{x} 8^{*} \mathrm{y} 2^{*} \mathrm{x} 1+270^{*} \mathrm{y} 1^{*} \mathrm{x} 2^{\wedge} 2+540^{*} \mathrm{x} 8^{*} \mathrm{x} 1^{*} \mathrm{x} 2+360^{*} \mathrm{x} 8^{*} \mathrm{x} 0^{*} \mathrm{x} 3+720^{*} \mathrm{y} 0^{*} \mathrm{y} 2^{*} \mathrm{x} 2- \\
& 360^{*} \mathrm{y} 2^{*} \mathrm{x} 0^{*} \mathrm{x} 2-180^{*} \mathrm{y} 1^{*} \mathrm{x} 8^{*} \mathrm{x} 2+1260^{*} \mathrm{y} 0^{*} \mathrm{x} 2^{*} \mathrm{x} 3+125^{*} \mathrm{x} 3^{\wedge} 2^{*} \mathrm{x} 1-100^{*} \mathrm{x} 2^{*} \mathrm{x} 0^{*} \mathrm{x} 3-
\end{aligned}
$$


$1530^{*} \mathrm{x} 0^{*} \mathrm{x} 8^{*} \mathrm{x} 2-425^{*} \mathrm{x} 3^{*} \mathrm{x} 2^{*} \mathrm{x} 1+270^{*} \mathrm{x} 3^{*} \mathrm{x} 8^{*} \mathrm{x} 1-270^{*} \mathrm{y} 2^{*} \mathrm{x} 2{ }^{*} \mathrm{x} 1-120^{*} \mathrm{x} 3^{\wedge} 3+$ $140^{*} \mathrm{x} 0 * \mathrm{x} 11^{\wedge} 2-540^{*} \mathrm{y} 0 * \mathrm{x} 2{ }^{\wedge} 2-360^{*} \mathrm{y} 0 * \mathrm{x} 3{ }^{\wedge} 2+90^{*} \mathrm{x} 2{ }^{\wedge} 3-40^{*} \mathrm{x} 1{ }^{\wedge} 3-80^{*} \mathrm{x} 0 \wedge 3+$ $120^{*} \mathrm{y} 00^{*} \mathrm{x} 0^{*} \mathrm{x} 2+240^{*} \mathrm{y} 0 * \mathrm{x} 1{ }^{*} \mathrm{x} 2-120^{*} \mathrm{y} 1{ }^{*} \mathrm{x} 1{ }^{*} \mathrm{x} 2+240^{*} \mathrm{x} 22^{*} \mathrm{x} 88^{*} \mathrm{x} 3+120^{*} \mathrm{y} 1{ }^{*} \mathrm{x} 00^{*} \mathrm{x} 3-$ $660^{*} \mathrm{x} 0^{*} \mathrm{x} 1^{*} \mathrm{x} 2+270^{*} \mathrm{x} 3^{*} \mathrm{x} 0^{*} \mathrm{x} 1+720^{*} \mathrm{x} 8^{\wedge} 2^{*} \mathrm{x} 0-630^{*} \mathrm{y} 1^{*} \mathrm{x} 2{ }^{*} \mathrm{x} 3-360^{*} \mathrm{y} 1^{*} \mathrm{y} 2^{*} \mathrm{x} 2+$ $720 * \mathrm{y} 1{ }^{*} \mathrm{y} 2 * \mathrm{x} 3+990^{*} \mathrm{y} 2 * \mathrm{x} 1 * \mathrm{x} 3-720 * \mathrm{y} 0 * \mathrm{x} 8{ }^{*} \mathrm{x} 3+625^{*} \mathrm{x} 0{ }^{*} \mathrm{x} 22^{\wedge} 2-180^{*} \mathrm{y} 2{ }^{*} \mathrm{x} 0 * \mathrm{x} 3+$ $360 * \mathrm{y} 0 * \mathrm{x} 8 * \mathrm{x} 2-1440 * \mathrm{y} 0 * \mathrm{y} 2 * \mathrm{x} 3+1080 * \mathrm{x} 8 * \mathrm{y} 2{ }^{*} \mathrm{x} 0+360 * \mathrm{y} 1 * \mathrm{x} 8 * \mathrm{x} 3-60 * \mathrm{y} 1{ }^{*} \mathrm{x} 0 * \mathrm{x} 2+$ $300^{*} \mathrm{y} 2{ }^{*} \mathrm{x} 0{ }^{*} \mathrm{x} 1+360^{*} \mathrm{y} 2{ }^{\wedge} 2^{*} \mathrm{x} 1+180^{*} \mathrm{y} 1{ }^{*} \mathrm{x} 3^{\wedge} 2-100^{*} \mathrm{x} 1^{*} \mathrm{x} 2{ }^{\wedge} 2-360^{*} \mathrm{x} 8^{\wedge} 2^{*} \mathrm{x} 1-720^{*} \mathrm{y} 2{ }^{\wedge}{ }^{*} \mathrm{x} 0$ $+480 * \mathrm{x} 2{ }^{*} \mathrm{y} 2 * \mathrm{x} 3+600 * \mathrm{x} 8 * \mathrm{x} 1{ }^{*} \mathrm{x} 0-240 * \mathrm{y} 0 * \mathrm{x} 0 * \mathrm{x} 3-480 * \mathrm{y} 0 * \mathrm{x} 1{ }^{*} \mathrm{x} 3+240 * \mathrm{y} 1{ }^{*} \mathrm{x} 1 *^{*} \mathrm{x} 3-$ $50^{*} \mathrm{x} 0^{*} \mathrm{x} 3^{\wedge} 2-300^{*} \mathrm{x} 1^{\wedge} 2^{*} \mathrm{x} 8+480^{*} \mathrm{x} 2^{*} \mathrm{x} 3^{\wedge} 2-600^{*} \mathrm{y} 2^{*} \mathrm{x} 0^{\wedge} 2-390^{*} \mathrm{x} 22^{\wedge} 2^{*} \mathrm{x} 3-480^{*} \mathrm{x} 3^{\wedge} 2^{*} \mathrm{y} 2-$ $60^{*} \mathrm{x} 2^{\wedge} 2^{*} \mathrm{x} 8+210^{*} \mathrm{x} 0^{\wedge} 2^{*} \mathrm{x} 2+90^{*} \mathrm{x} 11^{\wedge} 2^{*} \mathrm{x} 2-120^{*} \mathrm{x} 2^{\wedge} 2^{*} \mathrm{y} 2+270^{*} \mathrm{x} 1^{\wedge} 2^{*} \mathrm{x} 3-80^{*} \mathrm{x} 0^{\wedge} 2^{*} \mathrm{x} 1-$ $120^{*} \mathrm{x} 00^{\wedge} 2^{*} \mathrm{x} 3-240^{*} \mathrm{x} 3^{\wedge} 2^{*} \mathrm{x} 8$

\section{B.2 Right 2A-chain Constraint Variety}

The five Equations of the right constraint variety are as follows. The equations as presented are all set equal to zero. See Section 5.1.2.

$$
\begin{aligned}
& \mathrm{R} 1:=\mathrm{x} 0 * \mathrm{y} 0+\mathrm{x} 1{ }^{*} \mathrm{y} 1+\mathrm{x} 2 * \mathrm{y} 2+\mathrm{x} 3 * \mathrm{x} 8 \\
& \mathrm{R} 2:=-6623795100^{*} \mathrm{x} 2{ }^{*} \mathrm{y} 2+2752216116^{*} \mathrm{y} 2 * \mathrm{x} 3-2752216116^{*} \mathrm{y} 1{ }^{*} \mathrm{x} 0+2071988044^{*} \mathrm{x} 0{ }^{*} \mathrm{x} 1 \\
& -2071988044^{*} \mathrm{x} 3^{*} \mathrm{x} 2-2752216116^{*} \mathrm{y} 0^{*} \mathrm{x} 1+15391540705^{*} \mathrm{x} 3^{\wedge} 2+15391540705^{*} \mathrm{x} 0 \wedge 2+ \\
& 2752216116^{*} \mathrm{x} 8{ }^{*} \mathrm{x} 2+4415198112^{*} \mathrm{x} 3{ }^{*} \mathrm{y} 1+4415198112^{*} \mathrm{x} 2{ }^{*} \mathrm{y} 0-17176603205^{*} \mathrm{x} 1{ }^{\wedge} 2- \\
& 17176603205^{*} \mathrm{x} 22^{\wedge} 2-5901047008^{*} \mathrm{x} 1^{*} \mathrm{x} 3-4086579900^{*} \mathrm{x} 0 * \mathrm{y} 0-6623795100^{*} \mathrm{x} 1{ }^{*} \mathrm{y} 1- \\
& 4086579900 *_{\mathrm{x}} 3 *_{\mathrm{x} 8}+4415198112{ }^{*} \mathrm{x} 1 *_{\mathrm{x}} 8+441519811{ }^{*} \mathrm{x} 0{ }^{*} \mathrm{y} 2-5901047008 *_{\mathrm{x} 0}{ }^{*} \mathrm{x} 2 \text {; } \\
& \mathrm{R} 3:=-458702686^{*} \mathrm{x} 00^{*} \mathrm{x} 1+458702686^{*} \mathrm{x} 3^{*} \mathrm{x} 2+277357925^{*} \mathrm{x} 3^{\wedge} 2+277357925^{*} \mathrm{x} 00^{\wedge} 2+ \\
& 65923325^{*} \mathrm{x} 1^{\wedge} 2+65923325^{*} \mathrm{x} 2 \wedge 2+735866352^{*} \mathrm{x} 1^{*} \mathrm{x} 3+735866352^{*} \mathrm{x} 0^{*} \mathrm{x} 2 \\
& \mathrm{R} 4:=-424863351802500 * \mathrm{x} 2{ }^{*} \mathrm{y} 2+2110330638818220^{*} \mathrm{y} 2 * \mathrm{x} 3-2110330638818220^{*} \mathrm{y} 1{ }^{*} \mathrm{x} 0+ \\
& 9871064080132324 * \mathrm{x} 0 * \mathrm{x} 1-9930434915812324 * \mathrm{x} 3 * \mathrm{x} 2+3032245408295220 * \mathrm{y} 0 * \mathrm{x} 1+ \\
& 4760635983898475^{*} \times 3^{\wedge} 2+4696234126784075^{*} \times 0^{\wedge} 2-3032245408295220^{*} \times 8^{*} \mathrm{x} 2+ \\
& 592491708900000^{*} \mathrm{y} 0 \wedge 2+493540316100000^{*} \mathrm{y} 1^{\wedge} 2+592491708900000^{*} \mathrm{x} 8^{\wedge} 2+ \\
& 344385452736000 * \mathrm{y} 0 * \mathrm{y} 2+344385452736000 * \mathrm{y} 1 * \mathrm{x} 8-214672857048000 * \mathrm{y} 0 * \mathrm{y} 1+ \\
& 214672857048000^{*} \mathrm{y} 2 * \mathrm{x} 8-1401455043924000^{*} \mathrm{x} 2^{*} \mathrm{y} 1-2143739758836000^{*} \mathrm{x} 0 * \mathrm{x} 8+ \\
& 1510676487191040 * \mathrm{x} 3 * \mathrm{y} 1-3190656632255040 * \mathrm{x} 2 * \mathrm{y} 0+8688385849903625 * \mathrm{x} 1 \wedge 2+ \\
& 8623983992789225 * \times 2 \wedge 2+103315635820800 * \mathrm{x} 0 * \mathrm{x} 3-103315635820800 * \mathrm{x} 1{ }^{*} \mathrm{x} 2+ \\
& 5014752188102032 * \mathrm{x} 1 * \mathrm{x} 3+1764999023677500 * \mathrm{x} 0 * \mathrm{y} 0-424863351802500 * \mathrm{x} 1 * \mathrm{y} 1+ \\
& 1764999023677500 * \mathrm{x} 3 * \mathrm{x} 8+493540316100000 * \mathrm{y} 2 \wedge 2-3190656632255040 * \mathrm{x} 1{ }^{*} \mathrm{x} 8+ \\
& 1510676487191040 * \mathrm{x} 0 * \mathrm{y} 2+1401455043924000 * \mathrm{x} 1 * \mathrm{y} 2+2143739758836000 * \mathrm{x} 3 * \mathrm{y} 0+ \\
& 5014752188102032 * \mathrm{x} 0 * \mathrm{x} 2 \text {; } \\
& \mathrm{R} 5:=-36973056319875000^{*} \mathrm{x} 0 \wedge 2^{*} \mathrm{y} 0+52413286858569000^{*} \mathrm{x} 1{ }^{\wedge} 2^{*} \mathrm{y} 1+ \\
& 7428129303628800^{*} \mathrm{y} 2 \wedge 2^{*} \mathrm{x} 3+30299297043600000^{*} \mathrm{y} 00^{\wedge} 2^{*} \mathrm{x} 1+ \\
& 18887083096050000^{*} \mathrm{y} 0^{\wedge} 2^{*} \mathrm{x} 2-28044154563750000^{*} \mathrm{y} 0^{\wedge} 2^{*} \mathrm{x} 3+
\end{aligned}
$$


$33072924497558400 * \mathrm{y} 1 \wedge 2{ }^{*} \mathrm{x} 0-49616861955570000 * \mathrm{y} 1{ }^{\wedge} 2^{*} \mathrm{x} 2+$ $20616025260121200 * \mathrm{y} 1{ }^{\wedge} 2 * \mathrm{x} 3+30729778859520000 * \mathrm{x} 88^{\wedge} 2^{*} \mathrm{x} 2-$ $81529010851869000 * \mathrm{y} 1{ }^{*} \mathrm{x} 0 \wedge 2-30729778859520000 * \mathrm{y} 2 * \mathrm{x} 8{ }^{*} \mathrm{x} 3+$ $28044154563750000 * \mathrm{y} 0 * \mathrm{x} 8 * \mathrm{x} 0-11842695763470000 * \mathrm{y} 0 * \mathrm{x} 8 * \mathrm{x} 1+$ $150313090350581250^{*} \mathrm{x} 0{ }^{\wedge} 2^{*} \mathrm{x} 8+13187895956492400^{*} \mathrm{y} 1 * \mathrm{y} 2^{*} \mathrm{x} 0+$ $49616861955570000 * \mathrm{y} 1{ }^{*} \mathrm{y} 2 * \mathrm{x} 1+50731942289558400 * \mathrm{x} 8 *^{*} 2^{*} \mathrm{x} 1+$ $87841505853828000^{*} \mathrm{y} 1{ }^{*} \mathrm{x} 2 \wedge 2-140249774000490120{ }^{*} \mathrm{x} 8{ }^{*} \mathrm{x} 1{ }^{*} \mathrm{x} 2$ $3842249574825000 * \mathrm{x} 8{ }^{*} \mathrm{x} 0 * \mathrm{x} 3+4630328393558400 * \mathrm{y} 0 * \mathrm{y} 2 * \mathrm{x} 2-$ $26527355692387560 * \mathrm{y} 2 * \mathrm{x} 0 * \mathrm{x} 2+106952656027957560 * \mathrm{y} 0 * \mathrm{x} 1{ }^{\wedge} 2-$ $244262505609977250 * \mathrm{x} 1{ }^{\wedge} 2 * \mathrm{y} 2-13028689398441600 * \mathrm{y} 1{ }^{*} \mathrm{x} 8{ }^{*} \mathrm{x} 2+$ $213096045432636000 * \mathrm{y} 0{ }^{*} \mathrm{x} 2{ }^{*} \mathrm{x} 3-188267415226234925 * \mathrm{x} 3{ }^{\wedge} 2^{*} \mathrm{x} 1+$ $951746375427987450 * \mathrm{x} 2{ }^{*} \mathrm{x} 0 * \mathrm{x} 3+104315717906962560 * \mathrm{y} 1{ }^{*} \mathrm{x} 0 * \mathrm{x} 1-$ $51185017693467000 * \mathrm{x} 0 * \mathrm{x} 88^{*} \mathrm{x} 2+1183914950589925818^{*} \mathrm{x} 3{ }^{*} \mathrm{x} 2 * \mathrm{x} 1+$ $46256336508645000^{*} \mathrm{x} 3^{*} \mathrm{x} 8^{*} \mathrm{x} 1-35428218995259000^{*} \mathrm{y} 2^{*} \mathrm{x} 2{ }^{*} \mathrm{x} 1-$ $115654691230524000^{*} \mathrm{y} 0 * \mathrm{x} 1 * \mathrm{x} 0+7154841171292500^{*} \mathrm{x} 3^{\wedge} 3-$ $1417801127957369159 * \mathrm{x} 0 * \mathrm{x} 1 \wedge 2-18887083096050000^{*} \mathrm{y} 0 * \mathrm{y} 2 * \mathrm{x} 0-$ $33297117972532560 * \mathrm{y} 0 * \mathrm{x} 2{ }^{\wedge} 2-33130806745050000 * \mathrm{y} 0 * \mathrm{x} 33^{\wedge} 2-39705488594543100 * \mathrm{x} 22^{\wedge} 3-$ $33072924497558400^{*} \mathrm{y} 0 * \mathrm{y} 1{ }^{*} \mathrm{x} 1-247842389781694225^{*} \mathrm{x} 1{ }^{\wedge} 3-30299297043600000^{*} \mathrm{y} 0 * \mathrm{y} 1{ }^{*} \mathrm{x} 0$ - $139431272752620625^{*} \mathrm{x} 0 \wedge 3+27818323522086750 * \mathrm{y} 0 * \mathrm{x} 0 * \mathrm{x} 2+$ $120216108642041910 * \mathrm{y} 0 * \mathrm{x} 1{ }^{*} \mathrm{x} 2+232490559889169250 * \mathrm{y} 1{ }^{*} \mathrm{x} 1{ }^{*} \mathrm{x} 2-$ $20616025260121200^{*} \mathrm{y} 1{ }^{*} \mathrm{x} 8{ }^{*} \mathrm{x} 1-236764991969640000 * \mathrm{x} 2 * \mathrm{x} 8 * \mathrm{x} 3-$ $71826868074855750 * \mathrm{y} 1{ }^{*} \mathrm{x} 0 * \mathrm{x} 3-423230401907960499{ }^{*} \mathrm{x} 0{ }^{*} \mathrm{x} 1{ }^{*} \mathrm{x} 2-$ $171947047864268475^{*} \mathrm{x} 3^{*} \mathrm{x} 0{ }^{*} \mathrm{x} 1+118434802590000^{*} \mathrm{y} 1{ }^{*} \mathrm{x} 8^{*} \mathrm{x} 0-$ $38027664570121200 * \mathrm{y} 0 * \mathrm{y} 2 * \mathrm{x} 1+11724260960880000 * \mathrm{y} 0 * \mathrm{y} 1 * \mathrm{x} 3+$ $24839768613628800^{*} \mathrm{y} 0 * \mathrm{y} 1 * \mathrm{x} 2-28442596104000000^{*} \mathrm{x} 8{ }^{\wedge} 2^{*} \mathrm{x} 0-$ $280234423389562560 * \mathrm{y} 1 * \mathrm{x} 2 * \mathrm{x} 3+11143872876240000 * \mathrm{y} 1{ }^{*} \mathrm{y} 2 * \mathrm{x} 2-$ $37703252891116800^{*} \mathrm{y} 1 * \mathrm{y} 2 * \mathrm{x} 3-7428129303628800 * \mathrm{y} 2 * \mathrm{x} 88^{*} \mathrm{x} 2+$ $149391349790212440 * \mathrm{y} 2 * \mathrm{x} 1 * \mathrm{x} 3+28442596104000000^{*} \mathrm{y} 0 * \mathrm{x} 8 * \mathrm{x} 3-$ $239665858765713341 * \mathrm{x} 0 * \mathrm{x} 2 \wedge 2+124474282462809000 * \mathrm{y} 2 * \mathrm{x} 0 * \mathrm{x} 3-$ $49454721210960000 * \mathrm{y} 0 * \mathrm{x} 8 * \mathrm{x} 2+23424035019840000 * \mathrm{y} 0 * \mathrm{y} 2 * \mathrm{x} 3+$ $26030686191120000 * \mathrm{x} 8 * \mathrm{y} 2 * \mathrm{x} 0+19155424167360000 * \mathrm{y} 1 * \mathrm{x} 8 * \mathrm{x} 3+$ $211810577444520330 * \mathrm{y} 1 * \mathrm{x} 0 * \mathrm{x} 2-337693592790310920 * \mathrm{y} 2 * \mathrm{x} 0 * \mathrm{x} 1-$ $11143872876240000^{*} \mathrm{y} 2^{\wedge} 2^{*} \mathrm{x} 1+42945271610940000^{*} \mathrm{y} 1^{*} \mathrm{x} 3^{\wedge} 2-$ $233703669947525425^{*} \mathrm{x} 1{ }^{*} \mathrm{x} 2^{\wedge} 2-19155424167360000^{*} \mathrm{x} 8^{\wedge} 2^{*} \mathrm{x} 1-4630328393558400^{*} \mathrm{y} 22^{\wedge} 2^{*} \mathrm{x} 0$ $+81850517987785920^{*} \mathrm{x} 2 * \mathrm{y} 2 * \mathrm{x} 3+460330387274631000 * \mathrm{x} 88^{*} \mathrm{x} 1 * \mathrm{x} 0-$ $112814733776981250 * \mathrm{y} 0 * \mathrm{x} 0 * \mathrm{x} 3-195747071782904250 * \mathrm{y} 0 * \mathrm{x} 1 * \mathrm{x} 3-$ $44032497358004670^{*} \mathrm{y} 1{ }^{*} \mathrm{x} 1{ }^{*} \mathrm{x} 3-157976793206730625^{*} \mathrm{x} 0 * \mathrm{x} 3{ }^{\wedge} 2+$ $145300772460067830 * \times 1 \wedge 2 * x 8-50942947182774900 * \times 2 * \times 33^{\wedge} 2$ $85042346200935750^{*} \mathrm{y} 2^{*} \mathrm{x} 0 \wedge 2+198512197257946812^{*} \mathrm{x} 22^{\wedge} 2^{*} \mathrm{x} 3-$ $13215478126080000^{*} \mathrm{x} 3^{\wedge} 2^{*} \mathrm{y} 2+25084663818025920^{*} \mathrm{x} 2{ }^{\wedge} 2^{*} \mathrm{x} 8-$ $218902801826887125^{*} \mathrm{x} 0^{\wedge} 2^{*} \mathrm{x} 2-44650949578227750^{*} \mathrm{x} 1^{\wedge} 2^{*} \mathrm{x} 2-$ $11771945720808000^{*} \mathrm{x} 2{ }^{\wedge} 2^{*} \mathrm{y} 2-250451597095342437^{*} \mathrm{x} 1{ }^{\wedge} 2^{*} \mathrm{x} 3-$ $1144057632521222375^{*} \mathrm{x} 0^{\wedge} 2^{*} \mathrm{x} 1+2713830259991250^{*} \mathrm{x} 0{ }^{\wedge} 2^{*} \mathrm{x} 3+$ $37498356573600000^{*} \mathrm{x} 3^{\wedge} 2^{*} \mathrm{y} 3$; 


\section{B.3 Joint Variables of Left 2A-chain}

The equations for obtaining the joint variables of the left $2 \mathrm{~A}$-chain are as follows. The equations as presented are all set equal to zero. See Section 5.1.2. $u[1]$ and $u[2]$ represent joint variables $u_{1}$ and $u_{2}$ respectively.

$\mathrm{EQ1}:=\left(150.4225353^{*} \mathrm{u}[1]^{*} \mathrm{u}[2]^{\wedge} 3+150.4225353^{*} \mathrm{u}[1]^{\wedge} 3^{*} \mathrm{u}[2]-150.4225353^{*} \mathrm{u}[1]^{\wedge} 3^{*} \mathrm{u}[2]^{\wedge} 3\right.$ $+6.760563384^{*} \mathrm{u}[2]^{\wedge} 4-13.52112677^{*} \mathrm{u}[1]^{\wedge} 2-13.52112677^{*} \mathrm{u}[2]^{\wedge} 2+$ $331.2676070^{*} \mathrm{u}[1]^{\wedge} 2^{*} \mathrm{u}[2]^{\wedge} 2-13.52112677^{*} \mathrm{u}[1]^{\wedge} 2^{*} \mathrm{u}[2]^{\wedge} 4-13.52112677^{*} \mathrm{u}[1]^{\wedge} 4^{*} \mathrm{u}[2]^{\wedge} 2+$ $\left.6.760563384^{*} \mathrm{u}[1]^{\wedge} 4^{*} \mathrm{u}[2]^{\wedge} 4+6.760563384^{*} \mathrm{u}[1]^{\wedge} 4-150.4225353^{*} \mathrm{u}[1]^{*} \mathrm{u}[2]+6.760563384\right)$ $/\left(40 .^{*} \mathrm{u}[1]^{*} \mathrm{u}[2]^{\wedge} 3+40 .^{*} \mathrm{u}[1]^{\wedge} 3^{*} \mathrm{u}[2]-40 .^{*} \mathrm{u}[1]^{\wedge} 3^{*} \mathrm{u}[2]^{\wedge} 3+\mathrm{u}[2]^{\wedge} 4+1 .-2 .^{*} \mathrm{u}[1]^{\wedge} 2-\right.$ $2 .^{*} u[2]^{\wedge} 2+404 .^{*} u[1]^{\wedge} 2^{*} u[2]^{\wedge} 2-2 .^{*} u[1]^{\wedge} 2^{*} u[2]^{\wedge} 4-2 .^{*} u[1]^{\wedge} 4^{*} u[2]^{\wedge} 2+u[1]^{\wedge} 4^{*} u[2]^{\wedge} 4+$ $\left.\mathrm{u}[1]^{\wedge} 4-40 .^{*} \mathrm{u}[1]^{*} \mathrm{u}[2]\right)$;

$\mathrm{EQ} 2:=81.12676088^{*} \mathrm{u}[1]^{*} \mathrm{u}[2]^{\wedge} 3+81.12676088^{*} \mathrm{u}[1]^{\wedge} 3^{*} \mathrm{u}[2]-81.12676088^{*} \mathrm{u}[1]^{\wedge} 3^{*} \mathrm{u}[2]^{\wedge} 3-$ $9.999999999^{*} \mathrm{u}[2]^{\wedge} 3-227.9999999^{*} \mathrm{u}[1]^{*} \mathrm{u}[2]^{\wedge} 2+14 .^{*} \mathrm{u}[1]^{*} \mathrm{u}[2]^{\wedge} 4+$ $227.9999999^{*} \mathrm{u}[1]^{\wedge} 3^{*} \mathrm{u}[2]^{\wedge} 2-14 .^{*} \mathrm{u}[1]^{\wedge} 3^{*} \mathrm{u}[2]^{\wedge} 4+2.028169022^{*} \mathrm{u}[2]^{\wedge} 4+9.999999999^{*} \mathrm{u}[2]-$ $4.056338044^{*} \mathrm{u}[1]^{\wedge} 2+14 .^{*} \mathrm{u}[1]-4.056338044^{*} \mathrm{u}[2]^{\wedge} 2-14 .^{*} \mathrm{u}[1]^{\wedge} 3+$ $819.3802849^{*} \mathrm{u}[1]^{\wedge} 2^{*} \mathrm{u}[2]^{\wedge} 2-4.056338044^{*} \mathrm{u}[1]^{\wedge} 2^{*} \mathrm{u}[2]^{\wedge} 4-4.056338044^{*} \mathrm{u}[1]^{\wedge} 4^{*} \mathrm{u}[2]^{\wedge} 2+$ $2.028169022^{*} \mathrm{u}[1]^{\wedge} 4^{*} \mathrm{u}[2]^{\wedge} 4+2.028169022^{*} \mathrm{u}[1]^{\wedge} 4-300 *^{*} \mathrm{u}[2]^{*} \mathrm{u}[1]^{\wedge} 2+300 *^{*} \mathrm{u}[1]^{\wedge} 2^{*} \mathrm{u}[2]^{\wedge} 3$ $+9.999999999^{*} \mathrm{u}[2]^{*} \mathrm{u}[1]^{\wedge} 4-9.999999999^{*} \mathrm{u}[1]^{\wedge} 4^{*} \mathrm{u}[2]^{\wedge} 3-81.12676088^{*} \mathrm{u}[1]^{*} \mathrm{u}[2]+$ $2.028169022) /\left(40 *^{*} \mathrm{u}[1]^{*} \mathrm{u}[2]^{\wedge} 3+40 *^{*} \mathrm{u}[1]^{\wedge} 3^{*} \mathrm{u}[2]-40{ }^{*} \mathrm{u}[1]^{\wedge} 3^{*} \mathrm{u}[2]^{\wedge} 3+\mathrm{u}[2]^{\wedge} 4+1\right.$. $2 .^{*} \mathrm{u}[1]^{\wedge} 2-2 .{ }^{*} \mathrm{u}[2]^{\wedge} 2+404 .^{*} \mathrm{u}[1]^{\wedge} 2^{*} \mathrm{u}[2]^{\wedge} 2-2 .{ }^{*} \mathrm{u}[1]^{\wedge} 2^{*} \mathrm{u}[2]^{\wedge} 4-2 .{ }^{*} \mathrm{u}[1]^{\wedge} 4^{*} \mathrm{u}[2]^{\wedge} 2+$ $\left.\mathrm{u}[1]^{\wedge} 4^{*} \mathrm{u}[2]^{\wedge} 4+\mathrm{u}[1]^{\wedge} 4-40{ }^{*} \mathrm{u}[1]^{*} \mathrm{u}[2]\right)$

$\mathrm{EQ3}:=\left(40.56338016^{*} \mathrm{u}[1]^{*} \mathrm{u}[2]^{\wedge} 3+40.56338016^{*} \mathrm{u}[1]^{\wedge} 3^{*} \mathrm{u}[2]-40.56338016^{*} \mathrm{u}[1]^{\wedge} 3^{*} \mathrm{u}[2]^{\wedge} 3-\right.$ $10 *^{*} \mathrm{u}[2]^{\wedge} 3-204 .^{*} \mathrm{u}[1]^{*} \mathrm{u}[2]^{\wedge} 2+2 .^{*} \mathrm{u}[1]^{*} \mathrm{u}[2]^{\wedge} 4+204 .^{*} \mathrm{u}[1]^{\wedge} 3^{*} \mathrm{u}[2]^{\wedge} 2-2 .^{*} \mathrm{u}[1]^{\wedge} 3^{*} \mathrm{u}[2]^{\wedge} 4+$ $1.014084504^{*} \mathrm{u}[2]^{\wedge} 4+10 *^{*} \mathrm{u}[2]-2.028169008^{*} \mathrm{u}[1]^{\wedge} 2+2 *^{*} \mathrm{u}[1]-2.028169008^{*} \mathrm{u}[2]^{\wedge} 2-$ $2 .^{*} \mathrm{u}[1]^{\wedge} 3+409.6901396^{*} \mathrm{u}[1]^{\wedge} 2^{*} \mathrm{u}[2]^{\wedge} 2-2.028169008^{*} \mathrm{u}[1]^{\wedge} 2^{*} \mathrm{u}[2]^{\wedge} 4-$ $2.028169008^{*} \mathrm{u}[1]^{\wedge} 4^{*} \mathrm{u}[2]^{\wedge} 2+1.014084504^{*} \mathrm{u}[1]^{\wedge} 4^{*} \mathrm{u}[2]^{\wedge} 4+1.014084504^{*} \mathrm{u}[1]^{\wedge} 4-$ $60.00000001^{*} \mathrm{u}[2]^{*} \mathrm{u}[1]^{\wedge} 2+60.00000001^{*} \mathrm{u}[1]^{\wedge} 2^{*} \mathrm{u}[2]^{\wedge} 3+10 .^{*} \mathrm{u}[2]^{*} \mathrm{u}[1]^{\wedge} 4-$ $\left.10 .^{*} \mathrm{u}[1]^{\wedge} 4^{*} \mathrm{u}[2]^{\wedge} 3-40.56338016^{*} \mathrm{u}[1]^{*} \mathrm{u}[2]+1.014084504\right) /\left(40 .^{*} \mathrm{u}[1]^{*} \mathrm{u}[2]^{\wedge} 3+\right.$ $40 *^{*} \mathrm{u}[1]^{\wedge} 3^{*} \mathrm{u}[2]-40 *^{*} \mathrm{u}[1]^{\wedge} 3^{*} \mathrm{u}[2]^{\wedge} 3+\mathrm{u}[2]^{\wedge} 4+1 .-2 *^{*} \mathrm{u}[1]^{\wedge} 2-2 .^{*} \mathrm{u}[2]^{\wedge} 2+$ $404 *^{*} \mathrm{u}[1]^{\wedge} 2^{*} \mathrm{u}[2]^{\wedge} 2-2 .^{*} \mathrm{u}[1]^{\wedge} 2^{*} \mathrm{u}[2]^{\wedge} 4-2 *^{*} \mathrm{u}[1]^{\wedge} 4^{*} \mathrm{u}[2]^{\wedge} 2+\mathrm{u}[1]^{\wedge} 4^{*} \mathrm{u}[2]^{\wedge} 4+\mathrm{u}[1]^{\wedge} 4-$ $\left.40{ }^{*} \mathrm{u}[1]^{*} \mathrm{u}[2]\right)$

$\mathrm{EQ4}:=\left(-60 *^{*} \mathrm{u}[1]^{*} \mathrm{u}[2]^{\wedge} 3-60 *^{*} \mathrm{u}[1]^{\wedge} 3^{*} \mathrm{u}[2]-.6666666666^{*} \mathrm{u}[2]^{\wedge} 5-3.333333333^{*} \mathrm{u}[1]^{\wedge} 5+\right.$ $17.23943663^{*} \mathrm{u}[1]^{\wedge} 6-16.66666666^{*} \mathrm{u}[1]^{*} \mathrm{u}[2]^{\wedge} 2-16.66666666^{*} \mathrm{u}[1]^{*} \mathrm{u}[2]^{\wedge} 4+$ $389.5774653^{*} \mathrm{u}[2]^{*} \mathrm{u}[1]^{\wedge} 5+60 . * \mathrm{u}[1]^{\wedge} 5^{*} \mathrm{u}[2]^{\wedge} 3-19.23943663^{*} \mathrm{u}[2]^{\wedge} 4-$ $15.23943663^{*} \mathrm{u}[1]^{\wedge} 2^{*} \mathrm{u}[2]^{\wedge} 6-19.23943663^{*} \mathrm{u}[1]^{\wedge} 4^{*} \mathrm{u}[2]^{\wedge} 6+389.5774653^{*} \mathrm{u}[1]^{*} \mathrm{u}[2]^{\wedge} 5+$ $60 *^{*} \mathrm{u}[1]^{\wedge} 3^{*} \mathrm{u}[2]^{\wedge} 5-409.5774653^{*} \mathrm{u}[1]^{\wedge} 5^{*} \mathrm{u}[2]^{\wedge} 5-15.23943663^{*} \mathrm{u}[2]^{\wedge} 2^{*} \mathrm{u}[1]^{\wedge} 6-$ $19.23943663^{*} \mathrm{u}[2]^{\wedge} 4^{*} \mathrm{u}[1]^{\wedge} 6+17.23943663^{*} \mathrm{u}[1]^{\wedge} 6^{*} \mathrm{u}[2]^{\wedge} 6+3.333333333^{*} \mathrm{u}[2]^{\wedge} 6^{*} \mathrm{u}[1]+$ $67.33333333^{*} \mathrm{u}[2]^{\wedge} 5^{*} \mathrm{u}[1]^{\wedge} 2+67.33333333^{*} \mathrm{u}[2]^{\wedge} 5^{*} \mathrm{u}[1]^{\wedge} 4+16.66666666^{*} \mathrm{u}[1]^{\wedge} 5^{*} \mathrm{u}[2]^{\wedge} 2+$ $16.66666666^{*} \mathrm{u}[1]^{\wedge} 5^{*} \mathrm{u}[2]^{\wedge} 4+.6666666666^{*} \mathrm{u}[1]^{\wedge} 6^{*} \mathrm{u}[2]-3.333333333^{*} \mathrm{u}[1]^{\wedge} 5^{*} \mathrm{u}[2]^{\wedge} 6-$ $.6666666666^{*} \mathrm{u}[1]^{\wedge} 6^{*} \mathrm{u}[2]^{\wedge} 5+.6666666666^{*} \mathrm{u}[2]-15.23943663^{*} \mathrm{u}[1]^{\wedge} 2+3.333333333^{*} \mathrm{u}[1]-$ 
$15.23943663^{*} \mathrm{u}[2]^{\wedge} 2+17.23943663^{*} \mathrm{u}[2]^{\wedge} 6+509.0140900^{*} \mathrm{u}[1]^{\wedge} 2^{*} \mathrm{u}[2]^{\wedge} 2+$ $913.0140900^{*} \mathrm{u}[1]^{\wedge} 2^{*} \mathrm{u}[2]^{\wedge} 4+913.0140900^{*} \mathrm{u}[1]^{\wedge} 4^{*} \mathrm{u}[2]^{\wedge} 2+1317.014090^{*} \mathrm{u}[1]^{\wedge} 4^{*} \mathrm{u}[2]^{\wedge} 4-$ $19.23943663^{*} \mathrm{u}[1]^{\wedge} 4-67.33333333^{*} \mathrm{u}[2]^{*} \mathrm{u}[1]^{\wedge} 2-67.33333333^{*} \mathrm{u}[2]^{*} \mathrm{u}[1]^{\wedge} 4-$ $\left.369.5774652^{*} \mathrm{u}[1]^{*} \mathrm{u}[2]+17.23943663\right) /\left(\mathrm{u}[1]^{\wedge} 6+40 .^{*} \mathrm{u}[2]^{*} \mathrm{u}[1]^{\wedge} 5-1\right.$. $^{*} \mathrm{u}[2]^{\wedge} 4-$ 1.*u[1] ${ }^{\wedge} 2^{*} \mathrm{u}[2]^{\wedge} 6-1 *^{*} \mathrm{u}[1]^{\wedge} 4^{*} \mathrm{u}[2]^{\wedge} 6+40 .^{*} \mathrm{u}[1]^{*} \mathrm{u}[2]^{\wedge} 5-40 *^{*} \mathrm{u}[1]^{\wedge} 5^{*} \mathrm{u}[2]^{\wedge} 5-1 .^{*} \mathrm{u}[2]^{\wedge} 2^{*} \mathrm{u}[1]^{\wedge} 6$ $-1 *^{*} \mathrm{u}[2]^{\wedge} 4^{*} \mathrm{u}[1]^{\wedge} 6+\mathrm{u}[1]^{\wedge} 6^{*} \mathrm{u}[2]^{\wedge} 6-1 *^{*} \mathrm{u}[1]^{\wedge} 2-1 *^{*} \mathrm{u}[2]^{\wedge} 2+\mathrm{u}[2]^{\wedge} 6+401 *^{*} \mathrm{u}[1]^{\wedge} 2^{*} \mathrm{u}[2]^{\wedge} 2+$

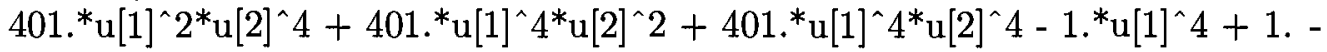
$\left.40{ }^{*} \mathrm{u}[1]{ }^{*} \mathrm{u}[2]\right)$

EQ5:= - $\left(300.0000001^{*} \mathrm{u}[1]^{*} \mathrm{u}[2]^{\wedge} 3+300.0000001^{*} \mathrm{u}[1]^{\wedge} 3^{*} \mathrm{u}[2]+4.666666665^{*} \mathrm{u}[2]^{\wedge} 5+\right.$ $3.333333335^{*} \mathrm{u}[1]^{\wedge} 5+2.704225338^{*} \mathrm{u}[1]^{\wedge} 6+96.66666665^{*} \mathrm{u}[1]^{*} \mathrm{u}[2]^{\wedge} 2+$ $96.66666665^{*} \mathrm{u}[1]^{*} \mathrm{u}[2]^{\wedge} 4+48.16901351^{*} \mathrm{u}[2]^{*} \mathrm{u}[1]^{\wedge} 5-300.0000001^{*} \mathrm{u}[1]^{\wedge} 5^{*} \mathrm{u}[2]^{\wedge} 3+$ $11.29577466^{*} \mathrm{u}[2]^{\wedge} 4-16.70422534^{*} \mathrm{u}[1]^{\wedge} 2^{*} \mathrm{u}[2]^{\wedge} 6+11.29577466^{*} \mathrm{u}[1]^{\wedge} 4^{*} \mathrm{u}[2]^{\wedge} 6+$ $48.16901351 * \mathrm{u}[1]{ }^{*} \mathrm{u}[2]^{\wedge} 5-300.0000001 *^{*} \mathrm{u}[1]^{\wedge} 3^{*} \mathrm{u}[2]^{\wedge} 5-28.16901349 *^{*} \mathrm{u}[1]^{\wedge} 5^{*} \mathrm{u}[2]^{\wedge} 5-$ $16.70422534^{*} \mathrm{u}[2]^{\wedge} 2^{*} \mathrm{u}[1]^{\wedge} 6+11.29577466^{*} \mathrm{u}[2]^{\wedge} 4^{*} \mathrm{u}[1]^{\wedge} 6+2.704225338^{*} \mathrm{u}[1]^{\wedge} 6^{*} \mathrm{u}[2]^{\wedge} 6-$ $3.333333335^{*} \mathrm{u}[2]^{\wedge} 6^{*} \mathrm{u}[1]-71.33333333^{*} \mathrm{u}[2]^{\wedge} 5^{*} \mathrm{u}[1]^{\wedge} 2-71.33333333^{*} \mathrm{u}[2]^{\wedge} 5^{*} \mathrm{u}[1]^{\wedge} 4-$ $96.66666665^{*} \mathrm{u}[1]^{\wedge} 5^{*} \mathrm{u}[2]^{\wedge} 2-96.66666665^{*} \mathrm{u}[1]^{\wedge} 5^{*} \mathrm{u}[2]^{\wedge} 4-4.666666665^{*} \mathrm{u}[1]^{\wedge} 6^{*} \mathrm{u}[2]+$ $3.333333335^{*} \mathrm{u}[1]^{\wedge} 5^{*} \mathrm{u}[2]^{\wedge} 6+4.666666665^{*} \mathrm{u}[1]^{\wedge} 6^{*} \mathrm{u}[2]^{\wedge} 5-4.666666665^{*} \mathrm{u}[2]-$

$16.70422534^{*} \mathrm{u}[1]^{\wedge} 2-3.333333335^{*} \mathrm{u}[1]-16.70422534^{*} \mathrm{u}[2]^{\wedge} 2+2.704225338^{*} \mathrm{u}[2]^{\wedge} 6+$ $312.3943604^{*} \mathrm{u}[1]^{\wedge} 2^{*} \mathrm{u}[2]^{\wedge} 2-115.6056396^{*} \mathrm{u}[1]^{\wedge} 2^{*} \mathrm{u}[2]^{\wedge} 4-115.6056396^{*} \mathrm{u}[1]^{\wedge} 4^{*} \mathrm{u}[2]^{\wedge} 2$ $543.6056395^{*} \mathrm{u}[1]^{\wedge} 4^{*} \mathrm{u}[2]^{\wedge} 4+11.29577466^{*} \mathrm{u}[1]^{\wedge} 4+2.704225338+$ $\left.71.33333333^{*} \mathrm{u}[2]^{*} \mathrm{u}[1]^{\wedge} 2+71.33333333^{*} \mathrm{u}[2]^{*} \mathrm{u}[1]^{\wedge} 4-68.16901350^{*} \mathrm{u}[1]^{*} \mathrm{u}[2]\right) /\left(\mathrm{u}[1]^{\wedge} 6+\right.$ $40 *^{*} \mathrm{u}[2]^{*} \mathrm{u}[1]^{\wedge} 5-1 .^{*} \mathrm{u}[2]^{\wedge} 4-1 .^{*} \mathrm{u}[1]^{\wedge} 2^{*} \mathrm{u}[2]^{\wedge} 6-1 *^{*} \mathrm{u}[1]^{\wedge} 4^{*} \mathrm{u}[2]^{\wedge} 6+40 .^{*} \mathrm{u}[1]^{*} \mathrm{u}[2]^{\wedge} 5-$ $40 *^{*} \mathrm{u}[1]^{\wedge} 5^{*} \mathrm{u}[2]^{\wedge} 5-1 .^{*} \mathrm{u}[2]^{\wedge} 2^{*} \mathrm{u}[1]^{\wedge} 6-1 *^{*} \mathrm{u}[2]^{\wedge} 4^{*} \mathrm{u}[1]^{\wedge} 6+\mathrm{u}[1]^{\wedge} 6^{*} \mathrm{u}[2]^{\wedge} 6-1$ * $^{*} \mathrm{u}[1]^{\wedge} 2-$ $1{ }^{*} \mathrm{u}[2]^{\wedge} 2+\mathrm{u}[2]^{\wedge} 6+401 *^{*} \mathrm{u}[1]^{\wedge} 2^{*} \mathrm{u}[2]^{\wedge} 2+401 .^{*} \mathrm{u}[1]^{\wedge} 2^{*} \mathrm{u}[2]^{\wedge} 4+401 *^{*} \mathrm{u}[1]^{\wedge} 4^{*} \mathrm{u}[2]^{\wedge} 2+$ $\left.401 .^{*} \mathrm{u}[1]^{\wedge} 4^{*} \mathrm{u}[2]^{\wedge} 4-1 .^{*} \mathrm{u}[1]^{\wedge} 4+1 .-40{ }^{*} \mathrm{u}[1]^{*} \mathrm{u}[2]\right)$

$\mathrm{EQ6}:=-\left(15 *^{*} \mathrm{u}[2]^{\wedge} 5+3 *^{*} \mathrm{u}[1]^{\wedge} 5+1.126760570^{*} \mathrm{u}[1]^{\wedge} 6-10 *^{*} \mathrm{u}[2]^{\wedge} 3+61 *^{*} \mathrm{u}[1]^{*} \mathrm{u}[2]^{\wedge} 2+\right.$ $317.0000001^{*} \mathrm{u}[1]^{*} \mathrm{u}[2]^{\wedge} 4+414 .^{*} \mathrm{u}[1]^{\wedge} 3^{*} \mathrm{u}[2]^{\wedge} 2+414 .^{*} \mathrm{u}[1]^{\wedge} 3^{*} \mathrm{u}[2]^{\wedge} 4+$ $21.07042282^{*} \mathrm{u}[2]^{*} \mathrm{u}[1]^{\wedge} 5-1.126760570^{*} \mathrm{u}[2]^{\wedge} 4-1.126760570^{*} \mathrm{u}[1]^{\wedge} 2^{*} \mathrm{u}[2]^{\wedge} 6-$ $1.126760570^{*} \mathrm{u}[1]^{\wedge} 4^{*} \mathrm{u}[2]^{\wedge} 6+21.07042282^{*} \mathrm{u}[1]^{*} \mathrm{u}[2]^{\wedge} 5-21.07042282^{*} \mathrm{u}[1]^{\wedge} 5^{*} \mathrm{u}[2]^{\wedge} 5-$ $1.126760570^{*} \mathrm{u}[2]^{\wedge} 2^{*} \mathrm{u}[1]^{\wedge} 6-1.126760570^{*} \mathrm{u}[2]^{\wedge} 4^{*} \mathrm{u}[1]^{\wedge} 6+1.126760570^{*} \mathrm{u}[1]^{\wedge} 6^{*} \mathrm{u}[2]^{\wedge} 6+$ $11 .^{*} \mathrm{u}[2]^{\wedge} 6^{*} \mathrm{u}[1]-14 .^{*} \mathrm{u}[2]^{\wedge} 6^{*} \mathrm{u}[1]^{\wedge} 3+225 .^{*} \mathrm{u}[2]^{\wedge} 5^{*} \mathrm{u}[1]^{\wedge} 2-95.00000001^{*} \mathrm{u}[2]^{\wedge} 5^{*} \mathrm{u}[1]^{\wedge} 4-$ $75 .^{*} \mathrm{u}[1]^{\wedge} 5^{*} \mathrm{u}[2]^{\wedge} 2-331 .^{*} \mathrm{u}[1]^{\wedge} 5^{*} \mathrm{u}[2]^{\wedge} 4-5 *^{*} \mathrm{u}[1]^{\wedge} 6^{*} \mathrm{u}[2]-10{ }^{*} \mathrm{u}[1]^{\wedge} 6^{*} \mathrm{u}[2]^{\wedge} 3+$ $3 *^{*} \mathrm{u}[1]^{\wedge} 5^{*} \mathrm{u}[2]^{\wedge} 6+15 *^{*} \mathrm{u}[1]^{\wedge} 6^{*} \mathrm{u}[2]^{\wedge} 5-5 *^{*} \mathrm{u}[2]-1.126760570^{*} \mathrm{u}[1]^{\wedge} 2+11 .^{*} \mathrm{u}[1]-$ $1.126760570^{*} \mathrm{u}[2]^{\wedge} 2+1.126760570^{*} \mathrm{u}[2]^{\wedge} 6-14 .^{*} \mathrm{u}[1]^{\wedge} 3-28.16901110^{*} \mathrm{u}[1]^{\wedge} 2^{*} \mathrm{u}[2]^{\wedge} 2-$ $28.16901110^{*} \mathrm{u}[1]^{\wedge} 2^{*} \mathrm{u}[2]^{\wedge} 4-28.16901110^{*} \mathrm{u}[1]^{\wedge} 4^{*} \mathrm{u}[2]^{\wedge} 2-28.16901110^{*} \mathrm{u}[1]^{\wedge} 4^{*} \mathrm{u}[2]^{\wedge} 4-$ $1.126760570^{*} \mathrm{u}[1]^{\wedge} 4-235 .^{*} \mathrm{u}[2]^{*} \mathrm{u}[1]^{\wedge} 2+570 .^{*} \mathrm{u}[1]^{\wedge} 2^{*} \mathrm{u}[2]^{\wedge} 3+85 .^{*} \mathrm{u}[2]^{*} \mathrm{u}[1]^{\wedge} 4+$ $\left.570 .^{*} \mathrm{u}[1]^{\wedge} 4^{*} \mathrm{u}[2]^{\wedge} 3+1.126760570-21.07042282^{*} \mathrm{u}[1]^{*} \mathrm{u}[2]\right) /\left(\mathrm{u}[1]^{\wedge} 6+40{ }^{*} \mathrm{u}[2]^{*} \mathrm{u}[1]^{\wedge} 5-\right.$ $1 *^{*} \mathrm{u}[2]^{\wedge} 4-1 *^{*} \mathrm{u}[1]^{\wedge} 2^{*} \mathrm{u}[2]^{\wedge} 6-1 *^{*} \mathrm{u}[1]^{\wedge} 4^{*} \mathrm{u}[2]^{\wedge} 6+40 *^{*} \mathrm{u}[1]^{*} \mathrm{u}[2]^{\wedge} 5-40 *^{*} \mathrm{u}[1]^{\wedge} 5^{*} \mathrm{u}[2]^{\wedge} 5-$ $1 .^{*} \mathrm{u}[2]^{\wedge} 2^{*} \mathrm{u}[1]^{\wedge} 6-1 .^{*} \mathrm{u}[2]^{\wedge} 4^{*} \mathrm{u}[1]^{\wedge} 6+\mathrm{u}[1]^{\wedge} 6^{*} \mathrm{u}[2]^{\wedge} 6-1 .^{*} \mathrm{u}[1]^{\wedge} 2-1 .^{*} \mathrm{u}[2]^{\wedge} 2+\mathrm{u}[2]^{\wedge} 6+$ 401.*u[1]^ $2^{*} \mathrm{u}[2]^{\wedge} 2+401 .^{*} \mathrm{u}[1]^{\wedge} 2^{*} \mathrm{u}[2]^{\wedge} 4+401 .^{*} \mathrm{u}[1]^{\wedge} 4^{*} \mathrm{u}[2]^{\wedge} 2+401 .^{*} \mathrm{u}[1]^{\wedge} 4^{*} \mathrm{u}[2]^{\wedge} 4-$ 1.* $\left.\mathrm{u}[1]^{\wedge} 4+1 .-40{ }^{*} \mathrm{u}[1]^{*} \mathrm{u}[2]\right)$;

EQ7: $=\left(4.999999998^{*} \mathrm{u}[2]^{\wedge} 5-29 .^{*} \mathrm{u}[1]^{\wedge} 5+.2816901690^{*} \mathrm{u}[1]^{\wedge} 6+9.999999999^{*} \mathrm{u}[2]^{\wedge} 3+\right.$ $277 . * \mathrm{u}[1]^{*} \mathrm{u}[2]^{\wedge} 2+69 .^{*} \mathrm{u}[1]^{*} \mathrm{u}[2]^{\wedge} 4-401.9999999^{*} \mathrm{u}[1]^{\wedge} 3^{*} \mathrm{u}[2]^{\wedge} 2-$ $401.9999999^{*} \mathrm{u}[1]^{\wedge} 3^{*} \mathrm{u}[2]^{\wedge} 4+43.26760675^{*} \mathrm{u}[2]^{*} \mathrm{u}[1]^{\wedge} 5-.2816901690^{*} \mathrm{u}[2]^{\wedge} 4-$ 
$.2816901690^{*} \mathrm{u}[1]^{\wedge} 2^{*} \mathrm{u}[2]^{\wedge} 6-.2816901690^{*} \mathrm{u}[1]^{\wedge} 4^{*} \mathrm{u}[2]^{\wedge} 6+43.26760675^{*} \mathrm{u}[1]^{*} \mathrm{u}[2]^{\wedge} 5-$ $43.26760675^{*} \mathrm{u}[1]^{\wedge} 5^{*} \mathrm{u}[2]^{\wedge} 5-.2816901690^{*} \mathrm{u}[2]^{\wedge} 2^{*} \mathrm{u}[1]^{\wedge} 6-.2816901690^{*} \mathrm{u}[2]^{\wedge} 4^{*} \mathrm{u}[1]^{\wedge} 6+$ $.2816901690^{*} \mathrm{u}[1]^{\wedge} 6^{*} \mathrm{u}[2]^{\wedge} 6+27 .^{*} \mathrm{u}[2]^{\wedge} 6^{*} \mathrm{u}[1]+2 .^{*} \mathrm{u}[2]^{\wedge} 6^{*} \mathrm{u}[1]^{\wedge} 3+$ $514.9999999^{*} \mathrm{u}[2]^{\wedge} 5^{*} \mathrm{u}[1]^{\wedge} 2+595 *^{*} \mathrm{u}[2]^{\wedge} 5^{*} \mathrm{u}[1]^{\wedge} 4-275 *^{*} \mathrm{u}[1]^{\wedge} 5^{*} \mathrm{u}[2]^{\wedge} 2-$ $66.99999999^{*} \mathrm{u}[1]^{\wedge} 5^{*} \mathrm{u}[2]^{\wedge} 4-15 .^{*} \mathrm{u}[1]^{\wedge} 6^{*} \mathrm{u}[2]+9.999999999^{*} \mathrm{u}[1]^{\wedge} 6^{*} \mathrm{u}[2]^{\wedge} 3-$ $29 .{ }^{*} \mathrm{u}[1]^{\wedge} 5^{*} \mathrm{u}[2]^{\wedge} 6+4.999999998^{*} \mathrm{u}[1]^{\wedge} 6^{*} \mathrm{u}[2]^{\wedge} 5-15 *^{*} \mathrm{u}[2]-.2816901690^{*} \mathrm{u}[1]^{\wedge} 2+27 . * \mathrm{u}[1]-$ $.2816901690^{*} \mathrm{u}[2]^{\wedge} 2+.2816901690^{*} \mathrm{u}[2]^{\wedge} 6+2 .^{*} \mathrm{u}[1]^{\wedge} 3+752.9577575^{*} \mathrm{u}[1]^{\wedge} 2^{*} \mathrm{u}[2]^{\wedge} 2+$ $752.9577575^{*} \mathrm{u}[1]^{\wedge} 2^{*} \mathrm{u}[2]^{\wedge} 4+752.9577575^{*} \mathrm{u}[1]^{\wedge} 4^{*} \mathrm{u}[2]^{\wedge} 2+752.9577575^{*} \mathrm{u}[1]^{\wedge} 4^{*} \mathrm{u}[2]^{\wedge} 4-$ $.2816901690^{*} \mathrm{u}[1]^{\wedge} 4-505.0000001^{*} \mathrm{u}[2]^{*} \mathrm{u}[1]^{\wedge} 2-89.99999998^{*} \mathrm{u}[1]^{\wedge} 2^{*} \mathrm{u}[2]^{\wedge} 3-$ $584.9999999^{*} \mathrm{u}[2]^{*} \mathrm{u}[1]^{\wedge} 4-89.99999998^{*} \mathrm{u}[1]^{\wedge} 4^{*} \mathrm{u}[2]^{\wedge} 3-43.26760675^{*} \mathrm{u}[1]^{*} \mathrm{u}[2]+$ $.2816901690) /\left(\mathrm{u}[1]^{\wedge} 6+40 *^{*} \mathrm{u}[2]^{*} \mathrm{u}[1]^{\wedge} 5-1 *^{*} \mathrm{u}[2]^{\wedge} 4-1 .^{*} \mathrm{u}[1]^{\wedge} 2^{*} \mathrm{u}[2]^{\wedge} 6-1 *^{*} \mathrm{u}[1]^{\wedge} 4^{*} \mathrm{u}[2]^{\wedge} 6+\right.$ $40 *^{*} \mathrm{u}[1]^{*} \mathrm{u}[2]^{\wedge} 5-40{ }^{*} \mathrm{u}[1]^{\wedge} 5^{*} \mathrm{u}[2]^{\wedge} 5-1 *^{*} \mathrm{u}[2]^{\wedge} 2^{*} \mathrm{u}[1]^{\wedge} 6-1 *^{*} \mathrm{u}[2]^{\wedge} 4^{*} \mathrm{u}[1]^{\wedge} 6+\mathrm{u}[1]^{\wedge} 6^{*} \mathrm{u}[2]^{\wedge} 6-$ $1{ }^{*} \mathrm{u}[1]^{\wedge} 2-1 .^{*} \mathrm{u}[2]^{\wedge} 2+\mathrm{u}[2]^{\wedge} 6+401{ }^{*} \mathrm{u}[1]^{\wedge} 2^{*} \mathrm{u}[2]^{\wedge} 2+401 *^{*} \mathrm{u}[1]^{\wedge} 2^{*} \mathrm{u}[2]^{\wedge} 4+$ $\left.401 .^{*} \mathrm{u}[1]^{\wedge} 4^{*} \mathrm{u}[2]^{\wedge} 2+401 .^{*} \mathrm{u}[1]^{\wedge} 4^{*} \mathrm{u}[2]^{\wedge} 4-1 *^{*} \mathrm{u}[1]^{\wedge} 4+1 .-40{ }^{*} \mathrm{u}[1]^{*} \mathrm{u}[2]\right)$;

\section{B.4 Joint Variables of Right 2A-chain}

The equations for obtaining the joint variables of the right $2 \mathrm{~A}$ - chain are as follows. The equations as presented are all set equal to zero. See Section 5.1.2. $u[3]$ and $u[4]$ represent joint variables $u_{3}$ and $u_{4}$ respectively.

$\mathrm{EQ1}:=-\left(-236.2499989^{*} \mathrm{u}[3]^{\wedge} 2+118.1249995^{*} \mathrm{u}[3]^{\wedge} 4-236.2499989^{*} \mathrm{u}[4]^{\wedge} 2+\right.$ $2122.499977^{*} \mathrm{u}[4]^{\wedge} 2^{*} \mathrm{u}[3]^{\wedge} 2-236.2499989^{*} \mathrm{u}[4]^{\wedge} 2^{*} \mathrm{u}[3]^{\wedge} 4-236.2499989^{*} \mathrm{u}[4]^{\wedge} 4^{*} \mathrm{u}[3]^{\wedge} 2+$ $118.1249995^{*} \mathrm{u}[4]^{\wedge} 4^{*} \mathrm{u}[3]^{\wedge} 4+1004.999993^{*} \mathrm{u}[3]^{*} \mathrm{u}[4]-1004.999993^{*} \mathrm{u}[4]^{*} \mathrm{u}[3]^{\wedge} 3-$ $1004.999993^{*} \mathrm{u}[3]^{*} \mathrm{u}[4]^{\wedge} 3+1004.999993^{*} \mathrm{u}[4]^{\wedge} 3^{*} \mathrm{u}[3]^{\wedge} 3+118.1249995^{*} \mathrm{u}[4]^{\wedge} 4+$ $118.1249995) /\left(-98 . * \mathrm{u}[3]^{\wedge} 2+49 .{ }^{*} \mathrm{u}[3]^{\wedge} 4-98 .^{*} \mathrm{u}[4]^{\wedge} 2+2132 .^{*} \mathrm{u}[4]^{\wedge} 2^{*} \mathrm{u}[3]^{\wedge} 2-\right.$ $98{ }^{*} \mathrm{u}[4]^{\wedge} 2^{*} \mathrm{u}[3]^{\wedge} 4-98 .^{*} \mathrm{u}[4]^{\wedge} 4^{*} \mathrm{u}[3]^{\wedge} 2+49 .^{*} \mathrm{u}[4]^{\wedge} 4^{*} \mathrm{u}[3]^{\wedge} 4+615.9999999^{*} \mathrm{u}[3]^{*} \mathrm{u}[4]-$ $615.9999999^{*} \mathrm{u}[4]^{*} \mathrm{u}[3]^{\wedge} 3-615.9999999^{*} \mathrm{u}[3]^{*} \mathrm{u}[4]^{\wedge} 3+615.9999999^{*} \mathrm{u}[4]^{\wedge} 3^{*} \mathrm{u}[3]^{\wedge} 3+$ 49. $\left.{ }^{*} \mathrm{u}[4]^{\wedge} 4+49.\right)$

$\mathrm{EQ} 2:=(-41.9999999)^{*} \mathrm{u}[4]-148.1484358^{*} \mathrm{u}[3]^{\wedge} 2+41.99999999 * \mathrm{u}[4]^{\wedge} 3+$ $74.07421790^{*} \mathrm{u}[3]^{\wedge} 4-148.1484358^{*} \mathrm{u}[4]^{\wedge} 2-125.9999999^{*} \mathrm{u}[3]+125.9999999^{*} \mathrm{u}[3]^{\wedge} 3+$ $3222.984338^{*} \mathrm{u}[4]^{\wedge} 2^{*} \mathrm{u}[3]^{\wedge} 2-148.1484358^{*} \mathrm{u}[4]^{\wedge} 2^{*} \mathrm{u}[3]^{\wedge} 4-148.1484358^{*} \mathrm{u}[4]^{\wedge} 4^{*} \mathrm{u}[3]^{\wedge} 2+$ $74.07421790^{*} \mathrm{u}[4]^{\wedge} 4^{*} \mathrm{u}[3]^{\wedge} 4-707.9999998^{*} \mathrm{u}[4]^{*} \mathrm{u}[3]^{\wedge} 2-41.9999999{ }^{*} \mathrm{u}[4]^{*} \mathrm{u}[3]^{\wedge} 4+$ $707.9999998^{*} \mathrm{u}[4]^{\wedge} 3^{*} \mathrm{u}[3]^{\wedge} 2+41.99999999^{*} \mathrm{u}[4]^{\wedge} 3^{*} \mathrm{u}[3]^{\wedge} 4-125.9999999^{*} \mathrm{u}[3]^{*} \mathrm{u}[4]^{\wedge} 4+$ $125.9999999^{*} \mathrm{u}[4]^{\wedge} 4^{*} \mathrm{u}[3]^{\wedge} 3-12{ }^{*} \mathrm{u}[3]^{*} \mathrm{u}[4]^{\wedge} 2+12 *^{*} \mathrm{u}[4]^{\wedge} 2^{*} \mathrm{u}[3]^{\wedge} 3+931.2187395 * \mathrm{u}[3]^{*} \mathrm{u}[4]-$ $931.2187395^{*} \mathrm{u}[4]^{*} \mathrm{u}[3]^{\wedge} 3-931.2187395^{*} \mathrm{u}[3]^{*} \mathrm{u}[4]^{\wedge} 3+931.2187395^{*} \mathrm{u}[4]^{\wedge} 3^{*} \mathrm{u}[3]^{\wedge} 3+$ $\left.74.07421790^{*} \mathrm{u}[4]^{\wedge} 4+74.07421790\right) /\left(-98 .^{*} \mathrm{u}[3]^{\wedge} 2+49 .^{*} \mathrm{u}[3]^{\wedge} 4-98 *^{*} \mathrm{u}[4]^{\wedge} 2+\right.$ $2132 .^{*} \mathrm{u}[4]^{\wedge} 2^{*} \mathrm{u}[3]^{\wedge} 2-98 .^{*} \mathrm{u}[4]^{\wedge} 2^{*} \mathrm{u}[3]^{\wedge} 4-98 .^{*} \mathrm{u}[4]^{\wedge} 4^{*} \mathrm{u}[3]^{\wedge} 2+49 .^{*} \mathrm{u}[4]^{\wedge} 4^{*} \mathrm{u}[3]^{\wedge} 4+$ $615.9999999^{*} \mathrm{u}[3]^{*} \mathrm{u}[4]-615.9999999^{*} \mathrm{u}[4]^{*} \mathrm{u}[3]^{\wedge} 3-615.9999999^{*} \mathrm{u}[3]^{*} \mathrm{u}[4]^{\wedge} 3+$ $615.9999999^{*} \mathrm{u}[4]^{\wedge} 3^{*} \mathrm{u}[3]^{\wedge} 3+49 .^{*} \mathrm{u}[4]^{\wedge} 4+49$.);

$\mathrm{EQ3}:=-\left(154 .^{*} \mathrm{u}[4]-191.7890606^{*} \mathrm{u}[3]^{\wedge} 2-154 .^{*} \mathrm{u}[4]^{\wedge} 3+95.89453032^{*} \mathrm{u}[3]^{\wedge} 4-\right.$ 
$191.7890606^{*} \mathrm{u}[4]^{\wedge} 2-98.00000003^{*} \mathrm{u}[3]+98.00000003^{*} \mathrm{u}[3]^{\wedge} 3+4172.390585^{*} \mathrm{u}[4]^{\wedge} 2^{*} \mathrm{u}[3]^{\wedge} 2$ $-191.7890606^{*} \mathrm{u}[4]^{\wedge} 2^{*} \mathrm{u}[3]^{\wedge} 4-191.7890606^{*} \mathrm{u}[4]^{\wedge} 4^{*} \mathrm{u}[3]^{\wedge} 2+95.89453032^{*} \mathrm{u}[4]^{\wedge} 4^{*} \mathrm{u}[3]^{\wedge} 4-$ $924.0000003^{*} \mathrm{u}[4]^{*} \mathrm{u}[3]^{\wedge} 2+154 . .^{*} \mathrm{u}[4]^{*} \mathrm{u}[3]^{\wedge} 4+924.0000003^{*} \mathrm{u}[4]^{\wedge} 3^{*} \mathrm{u}[3]^{\wedge} 2-$ $154 .^{*} \mathrm{u}[4]^{\wedge} 3^{*} \mathrm{u}[3]^{\wedge} 4-98.00000003^{*} \mathrm{u}[3]^{*} \mathrm{u}[4]^{\wedge} 4+98.00000003^{*} \mathrm{u}[4]^{\wedge} 4^{*} \mathrm{u}[3]^{\wedge} 3+$ $1164.000001^{*} \mathrm{u}[3]^{*} \mathrm{u}[4]^{\wedge} 2-1164.000001^{*} \mathrm{u}[4]^{\wedge} 2^{*} \mathrm{u}[3]^{\wedge} 3+1205.531238^{*} \mathrm{u}[3]^{*} \mathrm{u}[4]-$ $1205.531238^{*} \mathrm{u}[4]^{*} \mathrm{u}[3]^{\wedge} 3-1205.531238^{*} \mathrm{u}[3]^{*} \mathrm{u}[4]^{\wedge} 3+1205.531238^{*} \mathrm{u}[4]^{\wedge} 3^{*} \mathrm{u}[3]^{\wedge} 3+$ $\left.95.89453032^{*} u[4]^{\wedge} 4+95.89453032\right) /\left(-98 .^{*} u[3]^{\wedge} 2+49 .^{*} u[3]^{\wedge} 4-98 *^{*} u[4]^{\wedge} 2+\right.$ $2132 .^{*} \mathrm{u}[4]^{\wedge} 2^{*} \mathrm{u}[3]^{\wedge} 2-98 *^{*} \mathrm{u}[4]^{\wedge} 2^{*} \mathrm{u}[3]^{\wedge} 4-98 .^{*} \mathrm{u}[4]^{\wedge} 4^{*} \mathrm{u}[3]^{\wedge} 2+49 *^{*} \mathrm{u}[4]^{\wedge} 4^{*} \mathrm{u}[3]^{\wedge} 4+$ $615.9999999^{*} u[3]^{*} u[4]-615.9999999^{*} u[4]^{*} u[3]^{\wedge} 3-615.9999999^{*} u[3]^{*} u[4]^{\wedge} 3+$ $\left.615.9999999^{*} \mathrm{u}[4]^{\wedge} 3^{*} \mathrm{u}[3]^{\wedge} 3+49 .^{*} \mathrm{u}[4]^{\wedge} 4+49.\right)$

$\mathrm{EQ} 4:=-\left(133{ }^{*} \mathrm{u}[4]-384.0379189^{*} \mathrm{u}[3]^{\wedge} 2-188.0379189^{*} \mathrm{u}[3]^{\wedge} 4-133 .^{*} \mathrm{u}[4]^{\wedge} 5-\right.$ $6.99999999 *^{*} \mathrm{u}[3]^{\wedge} 5-384.0379189^{*} \mathrm{u}[4]^{\wedge} 6^{*} \mathrm{u}[3]^{\wedge} 2+88.99999999^{*} \mathrm{u}[4]^{\wedge} 5^{*} \mathrm{u}[3]^{\wedge} 2+$ $6.999999999 * \mathrm{u}[3]^{*} \mathrm{u}[4]^{\wedge} 6+88.9999999 *^{*} \mathrm{u}[4]^{\wedge} 5^{*} \mathrm{u}[3]^{\wedge} 4+924.000000{ }^{*} \mathrm{u}[4]^{\wedge} 5{ }^{*} \mathrm{u}[3]^{\wedge} 3-$ $384.0379189^{*} \mathrm{u}[3]^{\wedge} 6^{*} \mathrm{u}[4]^{\wedge} 2-188.0379189^{*} \mathrm{u}[3]^{\wedge} 6^{*} \mathrm{u}[4]^{\wedge} 4+133{ }^{*} \mathrm{u}[3]^{\wedge} 6^{*} \mathrm{u}[4]-$ $829.0000001^{*} \mathrm{u}[3]^{\wedge} 5^{*} \mathrm{u}[4]^{\wedge} 4-829.0000001^{*} \mathrm{u}[3]^{\wedge} 5^{*} \mathrm{u}[4]^{\wedge} 2-2557.905267^{*} \mathrm{u}[3]^{\wedge} 5^{*} \mathrm{u}[4]+$ $924.0000001^{*} \mathrm{u}[3]^{\wedge} 5^{*} \mathrm{u}[4]^{\wedge} 3-188.0379189^{*} \mathrm{u}[4]^{\wedge} 6^{*} \mathrm{u}[3]^{\wedge} 4+286.0379189^{*} \mathrm{u}[4]^{\wedge} 6^{*} \mathrm{u}[3]^{\wedge} 6-$ $6.999999999^{*} \mathrm{u}[4]^{\wedge} 6^{*} \mathrm{u}[3]^{\wedge} 5-133 .^{*} \mathrm{u}[4]^{\wedge} 5^{*} \mathrm{u}[3]^{\wedge} 6+2249.905267^{*} \mathrm{u}[4]^{\wedge} 5^{*} \mathrm{u}[3]^{\wedge} 5-$ $384.0379189^{*} \mathrm{u}[4]^{\wedge} 2+6.999999999^{*} \mathrm{u}[3]-2557.905267^{*} \mathrm{u}[3]^{*} \mathrm{u}[4]^{\wedge} 5+$ $7194.883042^{*} \mathrm{u}[4]^{\wedge} 2^{*} \mathrm{u}[3]^{\wedge} 2+5062.883044^{*} \mathrm{u}[4]^{\wedge} 2^{*} \mathrm{u}[3]^{\wedge} 4+5062.883044^{*} \mathrm{u}[4]^{\wedge} 4^{*} \mathrm{u}[3]^{\wedge} 2+$ $2930.883042^{*} \mathrm{u}[4]^{\wedge} 4^{*} \mathrm{u}[3]^{\wedge} 4-88.99999999^{*} \mathrm{u}[4]^{*} \mathrm{u}[3]^{\wedge} 2-88.99999999^{*} \mathrm{u}[4]^{*} \mathrm{u}[3]^{\wedge} 4+$ $829.0000001^{*} \mathrm{u}[3]^{*} \mathrm{u}[4]^{\wedge} 4+829.0000001^{*} \mathrm{u}[3]^{*} \mathrm{u}[4]^{\wedge} 2+2865.905267^{*} \mathrm{u}[3]^{*} \mathrm{u}[4]-$ $924.0000001^{*} \mathrm{u}[4]^{*} \mathrm{u}[3]^{\wedge} 3-924.0000001^{*} \mathrm{u}[3]^{*} \mathrm{u}[4]^{\wedge} 3+286.0379189^{*} \mathrm{u}[3]^{\wedge} 6+$ $\left.286.0379189^{*} \mathrm{u}[4]^{\wedge} 6-188.0379189^{*} \mathrm{u}[4]^{\wedge} 4+286.0379189\right) /\left(-49 .^{*} \mathrm{u}[3]^{\wedge} 2-49 .^{*} \mathrm{u}[3]^{\wedge} 4-\right.$ 49.*u[4]^ $6^{*} u[3]^{\wedge} 2-49 .^{*} \mathrm{u}[3]^{\wedge} 6^{*} \mathrm{u}[4]^{\wedge} 2-49 .^{*} \mathrm{u}[3]^{\wedge} 6^{*} \mathrm{u}[4]^{\wedge} 4-615.9999999^{*} \mathrm{u}[3]^{\wedge} 5^{*} \mathrm{u}[4]-$ $49{ }^{*} \mathrm{u}[4]^{\wedge} 6^{*} \mathrm{u}[3]^{\wedge} 4+49 . * \mathrm{u}[4]^{\wedge} 6^{*} \mathrm{u}[3]^{\wedge} 6+615.9999999^{*} \mathrm{u}[4]^{\wedge} 5^{*} \mathrm{u}[3]^{\wedge} 5-49 . * \mathrm{u}[4]^{\wedge} 2-$ $615.9999999^{*} \mathrm{u}[3]^{*} \mathrm{u}[4]^{\wedge} 5+49 .+1985 .^{*} \mathrm{u}[4]^{\wedge} 2^{*} \mathrm{u}[3]^{\wedge} 2+1985 .^{*} \mathrm{u}[4]^{\wedge} 2^{*} \mathrm{u}[3]^{\wedge} 4+$ 1985.* $\mathrm{u}[4]^{\wedge} 4^{*} \mathrm{u}[3]^{\wedge} 2+1985 .^{*} \mathrm{u}[4]^{\wedge} 4^{*} \mathrm{u}[3]^{\wedge} 4+615.9999999^{*} \mathrm{u}[3]^{*} \mathrm{u}[4]+49 .^{*} \mathrm{u}[3]^{\wedge} 6+$ $\left.49 *^{*} \mathrm{u}[4]^{\wedge} 6-49 *^{*} \mathrm{u}[4]^{\wedge} 4\right)$;

$\mathrm{EQ5}:=\left(-189.0000001^{*} \mathrm{u}[4]-235.6404431^{*} \mathrm{u}[3]^{\wedge} 2+109.6404431+16.35955690^{*} \mathrm{u}[3]^{\wedge} 4+\right.$ $189.0000001^{*} \mathrm{u}[4]^{\wedge} 5+231 .^{*} \mathrm{u}[3]^{\wedge} 5-235.6404431^{*} \mathrm{u}[4]^{\wedge} 6^{*} \mathrm{u}[3]^{\wedge} 2+1263 .^{*} \mathrm{u}[4]^{\wedge} 5^{*} \mathrm{u}[3]^{\wedge} 2$ $231 .{ }^{*} \mathrm{u}[3]^{*} \mathrm{u}[4]^{\wedge} 6+1263{ }^{*} \mathrm{u}[4]^{\wedge} 5^{*} \mathrm{u}[3]^{\wedge} 4+707.9999998^{*} \mathrm{u}[4]^{\wedge} 5^{*} \mathrm{u}[3]^{\wedge} 3-$ $235.6404431^{*} \mathrm{u}[3]^{\wedge} 6^{*} \mathrm{u}[4]^{\wedge} 2+16.35955690^{*} \mathrm{u}[3]^{\wedge} 6^{*} \mathrm{u}[4]^{\wedge} 4-189.0000001^{*} \mathrm{u}[3]^{\wedge} 6^{*} \mathrm{u}[4]+$ $957.0000001^{*} \mathrm{u}[3]^{\wedge} 5^{*} \mathrm{u}[4]^{\wedge} 4+957.0000001^{*} \mathrm{u}[3]^{\wedge} 5^{*} \mathrm{u}[4]^{\wedge} 2-1532.336999^{*} \mathrm{u}[3]^{\wedge} 5^{*} \mathrm{u}[4]+$ $707.9999998^{*} \mathrm{u}[3]^{\wedge} 5^{*} \mathrm{u}[4]^{\wedge} 3+16.35955690^{*} \mathrm{u}[4]^{\wedge} 6^{*} \mathrm{u}[3]^{\wedge} 4+109.6404431^{*} \mathrm{u}[4]^{\wedge} 6^{*} \mathrm{u}[3]^{\wedge} 6+$ $231 .^{*} \mathrm{u}[4]^{\wedge} 6^{*} \mathrm{u}[3]^{\wedge} 5+189.0000001^{*} \mathrm{u}[4]^{\wedge} 5^{*} \mathrm{u}[3]^{\wedge} 6+1616.337000^{*} \mathrm{u}[4]^{\wedge} 5^{*} \mathrm{u}[3]^{\wedge} 5-$ $235.6404431^{*} \mathrm{u}[4]^{\wedge} 2-231 .^{*} \mathrm{u}[3]-1532.336999^{*} \mathrm{u}[3]^{*} \mathrm{u}[4]^{\wedge} 5+5133.556728^{*} \mathrm{u}[4]^{\wedge} 2^{*} \mathrm{u}[3]^{\wedge} 2+$ $5409.556728^{*} \mathrm{u}[4]^{\wedge} 2^{*} \mathrm{u}[3]^{\wedge} 4+5409.556728^{*} \mathrm{u}[4]^{\wedge} 4^{*} \mathrm{u}[3]^{\wedge} 2+5685.556727^{*} \mathrm{u}[4]^{\wedge} 4^{*} \mathrm{u}[3]^{\wedge} 4-$ 1263.*u[4] ${ }^{*} \mathrm{u}[3]^{\wedge} 2-1263 .^{*} \mathrm{u}[4]^{*} \mathrm{u}[3]^{\wedge} 4-957.0000001^{*} \mathrm{u}[3]^{*} \mathrm{u}[4]^{\wedge} 4-957.0000001^{*} \mathrm{u}[3]^{*} \mathrm{u}[4]^{\wedge} 2$ $+1448.336999^{*} \mathrm{u}[3]^{*} \mathrm{u}[4]-707.9999998^{*} \mathrm{u}[4]^{*} \mathrm{u}[3]^{\wedge} 3-707.9999998^{*} \mathrm{u}[3]^{*} \mathrm{u}[4]^{\wedge} 3+$ $\left.109.6404431^{*} \mathrm{u}[3]^{\wedge} 6+109.6404431^{*} \mathrm{u}[4]^{\wedge} 6+16.35955690^{*} \mathrm{u}[4]^{\wedge} 4\right) /\left(-49 . * u[3]^{\wedge} 2-\right.$ $49 .^{*} \mathrm{u}[3]^{\wedge} 4-49 .^{*} \mathrm{u}[4]^{\wedge} 6^{*} \mathrm{u}[3]^{\wedge} 2-49 .^{*} \mathrm{u}[3]^{\wedge} 6^{*} \mathrm{u}[4]^{\wedge} 2-49 *^{*} \mathrm{u}[3]^{\wedge} 6^{*} \mathrm{u}[4]^{\wedge} 4-$

$615.9999999^{*} \mathrm{u}[3]^{\wedge} 5^{*} \mathrm{u}[4]-49 .^{*} \mathrm{u}[4]^{\wedge} 6^{*} \mathrm{u}[3]^{\wedge} 4+49 .^{*} \mathrm{u}[4]^{\wedge} 6^{*} \mathrm{u}[3]^{\wedge} 6+$ $615.9999999^{*} \mathrm{u}[4]^{\wedge} 5^{*} \mathrm{u}[3]^{\wedge} 5-49 .^{*} \mathrm{u}[4]^{\wedge} 2-615.9999999^{*} \mathrm{u}[3]^{*} \mathrm{u}[4]^{\wedge} 5+49 .+$ 1985. ${ }^{*} \mathrm{u}[4]^{\wedge} 2^{*} \mathrm{u}[3]^{\wedge} 2+1985 .{ }^{*} \mathrm{u}[4]^{\wedge} 2^{*} \mathrm{u}[3]^{\wedge} 4+1985{ }^{*} \mathrm{u}[4]^{\wedge} 4^{*} \mathrm{u}[3]^{\wedge} 2+1985 .^{*} \mathrm{u}[4]^{\wedge} 4^{*} \mathrm{u}[3]^{\wedge} 4+$ 
$\left.615.9999999^{*} \mathrm{u}[3]^{*} \mathrm{u}[4]+49 .^{*} \mathrm{u}[3]^{\wedge} 6+49 \cdot *^{*} \mathrm{u}[4]^{\wedge} 6-49 . * u[4]^{\wedge} 4\right)$

$\mathrm{EQ6}:=\left(290.5000000^{*} \mathrm{u}[4]-519.1514840^{*} \mathrm{u}[3]^{\wedge} 2-41.99999999^{*} \mathrm{u}[4]^{\wedge} 3-519.1514840^{*} \mathrm{u}[3]^{\wedge} 4\right.$ $-248.5000000^{*} \mathrm{u}[4]^{\wedge} 5+283.5000000^{*} \mathrm{u}[3]^{\wedge} 5-519.1514840^{*} \mathrm{u}[4]^{\wedge} 6^{*} \mathrm{u}[3]^{\wedge} 2+$ $1322.500000^{*} \mathrm{u}[4]^{\wedge} 5^{*} \mathrm{u}[3]^{\wedge} 2-157.5000000^{*} \mathrm{u}[3]^{*} \mathrm{u}[4]^{\wedge} 6+1946.500000^{*} \mathrm{u}[4]^{\wedge} 5^{*} \mathrm{u}[3]^{\wedge} 4-$ $519.1514840^{*} u[3]^{\wedge} 6^{*} u[4]^{\wedge} 2-519.1514840^{*} u[3]^{\wedge} 6^{*} u[4]^{\wedge} 4+290.5000000^{*} u[3]^{\wedge} 6^{*} u[4]-$ $41.99999999^{*} \mathrm{u}[3]^{\wedge} 6^{*} \mathrm{u}[4]^{\wedge} 3-2097.500000^{*} \mathrm{u}[3]^{\wedge} 5^{*} \mathrm{u}[4]^{\wedge} 4-1857.500000^{*} \mathrm{u}[3]^{\wedge} 5^{*} \mathrm{u}[4]^{\wedge} 2-$ $5422.475798^{*} \mathrm{u}[3]^{\wedge} 5^{*} \mathrm{u}[4]-519.1514840^{*} \mathrm{u}[4]^{\wedge} 6^{*} \mathrm{u}[3]^{\wedge} 4-126{ }^{*} \mathrm{u}[4]^{\wedge} 6^{*} \mathrm{u}[3]^{\wedge} 3+$ $519.1514840^{*} \mathrm{u}[4]^{\wedge} 6^{*} \mathrm{u}[3]^{\wedge} 6+283.5000000^{*} \mathrm{u}[4]^{\wedge} 6^{*} \mathrm{u}[3]^{\wedge} 5-248.5000000^{*} \mathrm{u}[4]^{\wedge} 5^{*} \mathrm{u}[3]^{\wedge} 6+$ $5422.475798^{*} \mathrm{u}[4]^{\wedge} 5^{*} \mathrm{u}[3]^{\wedge} 5-519.1514840^{*} \mathrm{u}[4]^{\wedge} 2-157.5000000^{*} \mathrm{u}[3]-126 .^{*} \mathrm{u}[3]^{\wedge} 3-$ $5422.475798^{*} \mathrm{u}[3]^{*} \mathrm{u}[4]^{\wedge} 5+14091.50399^{*} \mathrm{u}[4]^{\wedge} 2^{*} \mathrm{u}[3]^{\wedge} 2+14091.50399^{*} \mathrm{u}[4]^{\wedge} 2^{*} \mathrm{u}[3]^{\wedge} 4+$ $14091.50399^{*} \mathrm{u}[4]^{\wedge} 4^{*} \mathrm{u}[3]^{\wedge} 2+14091.50399^{*} \mathrm{u}[4]^{\wedge} 4^{*} \mathrm{u}[3]^{\wedge} 4-1364.500000^{*} \mathrm{u}[4]^{*} \mathrm{u}[3]^{\wedge} 2-$ 1988.500000* $\mathrm{u}[4]^{*} \mathrm{u}[3]^{\wedge} 4-1542 .^{*} \mathrm{u}[4]^{\wedge} 3^{*} \mathrm{u}[3]^{\wedge} 2-1542{ }^{*} \mathrm{u}[4]^{\wedge} 3^{*} \mathrm{u}[3]^{\wedge} 4+$ $1971.500000^{*} \mathrm{u}[3]^{*} \mathrm{u}[4]^{\wedge} 4-402 *^{*} \mathrm{u}[4]^{\wedge} 4^{*} \mathrm{u}[3]^{\wedge} 3+1731.500000^{*} \mathrm{u}[3]^{*} \mathrm{u}[4]^{\wedge} 2-$ $402{ }^{*} \mathrm{u}[4]^{\wedge} 2^{*} \mathrm{u}[3]^{\wedge} 3+5422.475798^{*} \mathrm{u}[3]^{*} \mathrm{u}[4]+519.1514840^{*} \mathrm{u}[3]^{\wedge} 6+519.1514840^{*} \mathrm{u}[4]^{\wedge} 6-$ $\left.519.1514840^{*} \mathrm{u}[4]^{\wedge} 4+519.1514840\right) /\left(-49 .^{*} \mathrm{u}[3]^{\wedge} 2-49 .^{*} \mathrm{u}[3]^{\wedge} 4-49 .^{*} \mathrm{u}[4]^{\wedge} 6^{*} \mathrm{u}[3]^{\wedge} 2-\right.$ $49 .^{*} \mathrm{u}[3]^{\wedge} 6^{*} \mathrm{u}[4]^{\wedge} 2-49 .^{*} \mathrm{u}[3]^{\wedge} 6^{*} \mathrm{u}[4]^{\wedge} 4-615.9999999^{*} \mathrm{u}[3]^{\wedge} 5^{*} \mathrm{u}[4]-49 .^{*} \mathrm{u}[4]^{\wedge} 6^{*} \mathrm{u}[3]^{\wedge} 4+$ $49 .^{*} \mathrm{u}[4]^{\wedge} 6^{*} \mathrm{u}[3]^{\wedge} 6+615.9999999^{*} \mathrm{u}[4]^{\wedge} 5^{*} \mathrm{u}[3]^{\wedge} 5-49 .^{*} \mathrm{u}[4]^{\wedge} 2-615.9999999^{*} \mathrm{u}[3]^{*} \mathrm{u}[4]^{\wedge} 5+$ 49. $+1985{ }^{*} \mathrm{u}[4]^{\wedge} 2^{*} \mathrm{u}[3]^{\wedge} 2+1985 .^{*} \mathrm{u}[4]^{\wedge} 2^{*} \mathrm{u}[3]^{\wedge} 4+1985 *^{*} \mathrm{u}[4]^{\wedge} 4^{*} \mathrm{u}[3]^{\wedge} 2+$ 1985.* $\left.\mathrm{u}[4]^{\wedge} 4^{*} \mathrm{u}[3]^{\wedge} 4+615.9999999^{*} \mathrm{u}[3]^{*} \mathrm{u}[4]+49 .^{*} \mathrm{u}[3]^{\wedge} 6+49 .^{*} \mathrm{u}[4]^{\wedge} 6-49 .^{*} \mathrm{u}[4]^{\wedge} 4\right)$

$\mathrm{EQ7}:=\left(3.500000000^{*} \mathrm{u}[4]-262.6265150^{*} \mathrm{u}[3]^{\wedge} 2-154 .^{*} \mathrm{u}[4]^{\wedge} 3-262.6265150^{*} \mathrm{u}[3]^{\wedge} 4+\right.$ $150.5000000^{*} \mathrm{u}[4]^{\wedge} 5+234.5000000^{*} \mathrm{u}[3]^{\wedge} 5-262.6265150^{*} \mathrm{u}[4]^{\wedge} 6^{*} \mathrm{u}[3]^{\wedge} 2+$ $2247.500000^{*} \mathrm{u}[4]^{\wedge} 5^{*} \mathrm{u}[3]^{\wedge} 2-332.5000001^{*} \mathrm{u}[3]^{*} \mathrm{u}[4]^{\wedge} 6+1015.500000^{*} \mathrm{u}[4]^{\wedge} 5^{*} \mathrm{u}[3]^{\wedge} 4-$ $262.6265150^{*} \mathrm{u}[3]^{\wedge} 6^{*} \mathrm{u}[4]^{\wedge} 2-262.6265150^{*} \mathrm{u}[3]^{\wedge} 6^{*} \mathrm{u}[4]^{\wedge} 4+3.500000000^{*} \mathrm{u}[3]^{\wedge} 6^{*} \mathrm{u}[4]-$ $154 .^{*} \mathrm{u}[3]^{\wedge} 6^{*} \mathrm{u}[4]^{\wedge} 3+907.5000001^{*} \mathrm{u}[3]^{\wedge} 5^{*} \mathrm{u}[4]^{\wedge} 4-452.4999999^{*} \mathrm{u}[3]^{\wedge} 5^{*} \mathrm{u}[4]^{\wedge} 2-$ $3013.590474^{*} \mathrm{u}[3]^{\wedge} 5^{*} \mathrm{u}[4]-262.6265150^{*} \mathrm{u}[4]^{\wedge} 6^{*} \mathrm{u}[3]^{\wedge} 4+98.00000001^{*} \mathrm{u}[4]^{\wedge} 6^{*} \mathrm{u}[3]^{\wedge} 3+$ $262.6265150^{*} \mathrm{u}[4]^{\wedge} 6^{*} \mathrm{u}[3]^{\wedge} 6+234.5000000^{*} \mathrm{u}[4]^{\wedge} 6^{*} \mathrm{u}[3]^{\wedge} 5+150.5000000^{*} \mathrm{u}[4]^{\wedge} 5^{*} \mathrm{u}[3]^{\wedge} 6+$ $3013.590474^{*} \mathrm{u}[4]^{\wedge} 5^{*} \mathrm{u}[3]^{\wedge} 5-262.6265150^{*} \mathrm{u}[4]^{\wedge} 2-332.5000001^{*} \mathrm{u}[3]+98.00000001^{*} \mathrm{u}[3]^{\wedge} 3$ $-3013.590474^{*} \mathrm{u}[3]^{*} \mathrm{u}[4]^{\wedge} 5+8828.768012^{*} \mathrm{u}[4]^{\wedge} 2^{*} \mathrm{u}[3]^{\wedge} 2+8828.768012^{*} \mathrm{u}[4]^{\wedge} 2^{*} \mathrm{u}[3]^{\wedge} 4+$ $8828.768012^{*} \mathrm{u}[4]^{\wedge} 4^{*} \mathrm{u}[3]^{\wedge} 2+8828.768012^{*} \mathrm{u}[4]^{\wedge} 4^{*} \mathrm{u}[3]^{\wedge} 4-2401.500000^{*} \mathrm{u}[4]^{*} \mathrm{u}[3]^{\wedge} 2$ $1169.500000^{*} \mathrm{u}[4]^{*} \mathrm{u}[3]^{\wedge} 4+1386 .^{*} \mathrm{u}[4]^{\wedge} 3^{*} \mathrm{u}[3]^{\wedge} 2+1386 .{ }^{*} \mathrm{u}[4]^{\wedge} 3^{*} \mathrm{u}[3]^{\wedge} 4-$ $809.5000002^{*} \mathrm{u}[3]^{*} \mathrm{u}[4]^{\wedge} 4-2034 .^{*} \mathrm{u}[4]^{\wedge} 4^{*} \mathrm{u}[3]^{\wedge} 3+550.5000000^{*} \mathrm{u}[3]^{*} \mathrm{u}[4]^{\wedge} 2-$ $2034 .{ }^{*} \mathrm{u}[4]^{\wedge} 2^{*} \mathrm{u}[3]^{\wedge} 3+3013.590474^{*} \mathrm{u}[3]^{*} \mathrm{u}[4]+262.6265150^{*} \mathrm{u}[3]^{\wedge} 6+262.6265150^{*} \mathrm{u}[4]^{\wedge} 6$ $\left.-262.6265150^{*} \mathrm{u}[4]^{\wedge} 4+262.6265150\right) /\left(-49 .{ }^{*} \mathrm{u}[3]^{\wedge} 2-49{ }^{*} \mathrm{u}[3]^{\wedge} 4-49 .^{*} \mathrm{u}[4]^{\wedge} 6^{*} \mathrm{u}[3]^{\wedge} 2-\right.$ $49 .^{*} \mathrm{u}[3]^{\wedge} 6^{*} \mathrm{u}[4]^{\wedge} 2-49 .^{*} \mathrm{u}[3]^{\wedge} 6^{*} \mathrm{u}[4]^{\wedge} 4-615.9999999^{*} \mathrm{u}[3]^{\wedge} 5^{*} \mathrm{u}[4]-49 .^{*} \mathrm{u}[4]^{\wedge} 6^{*} \mathrm{u}[3]^{\wedge} 4+$ $49 .{ }^{*} \mathrm{u}[4]^{\wedge} 6^{*} \mathrm{u}[3]^{\wedge} 6+615.9999999^{*} \mathrm{u}[4]^{\wedge} 5^{*} \mathrm{u}[3]^{\wedge} 5-49{ }^{*} \mathrm{u}[4]^{\wedge} 2-615.9999999^{*} \mathrm{u}[3]^{*} \mathrm{u}[4]^{\wedge} 5+$ 49. $+1985 *^{*} \mathrm{u}[4]^{\wedge} 2^{*} \mathrm{u}[3]^{\wedge} 2+1985 .^{*} \mathrm{u}[4]^{\wedge} 2^{*} \mathrm{u}[3]^{\wedge} 4+1985{ }^{*} \mathrm{u}[4]^{\wedge} 4^{*} \mathrm{u}[3]^{\wedge} 2+$ 1985. $\left.{ }^{*} \mathrm{u}[4]^{\wedge} 4^{*} \mathrm{u}[3]^{\wedge} 4+615.9999999^{*} \mathrm{u}[3]^{*} \mathrm{u}[4]+49 .^{*} \mathrm{u}[3]^{\wedge} 6+49 .{ }^{*} \mathrm{u}[4]^{\wedge} 6-49 .{ }^{*} \mathrm{u}[4]^{\wedge} 4\right)$ 


\section{Appendix C}

\section{Equations for 6A-manipulator Numeric Example}

In this Appendix equations for the numeric 6A-manipulator inverse kinematics example are presented. Note that because of the size of some of the equations in this section they are presented in the format in which they are input into the Maple algebraic mathematics software. The equations are presented without subscripts and with exponents represented by the character "^, and the following number (i.e. $\mathrm{x} 1^{\wedge} 2$ is equivalent to $x_{1}^{2}$ ). The fonts used may also vary from those in the text of this thesis.

\section{C.1 Left 3A-chain Constraint Variety}

The five Equations of the left constraint variety are as follows. The equations as presented are all set equal to zero. See Section 5.2.2. $\mathrm{u}[3]$ represent joint variable $u_{3}$.

$$
\begin{aligned}
& \mathrm{L} 1:=\mathrm{x} 0^{*} \mathrm{y} 0+\mathrm{x} 1^{*} \mathrm{y} 1+\mathrm{x} 2^{*} \mathrm{y} 2+\mathrm{x} 3^{*} \mathrm{y} 3 \\
& \mathrm{~L} 2:=960^{*} \mathrm{u}[3]^{\wedge} 2^{*} \mathrm{x} 2{ }^{*} \mathrm{y} 2+1560^{*} \mathrm{u}[3]^{\wedge} 2^{*} \mathrm{x} 2^{*} \mathrm{x} 0+311^{*} \mathrm{u}[3]^{\wedge} 2^{*} \mathrm{x} 2^{*} \mathrm{x} 3+196^{*} \mathrm{u}[3]^{\wedge} 6^{*} \mathrm{x} 3^{*} \mathrm{y} 3- \\
& 180^{*} \mathrm{u}[3]^{\wedge} 2^{*} \mathrm{x} 3^{*} \mathrm{y} 3+16^{*} \mathrm{u}[3]^{\wedge} 2^{*} \mathrm{y} 2^{*} \mathrm{x} 3-1560^{*} \mathrm{u}[3]^{\wedge} 2^{*} \mathrm{x} 1^{*} \mathrm{x} 3+16^{*} \mathrm{u}[3]^{\wedge} 2^{*} \mathrm{x} 2^{*} \mathrm{y} 3+ \\
& 112^{*} \mathrm{u}[3]^{\wedge} 6^{*} \mathrm{x} 2{ }^{*} \mathrm{y} 3+112^{*} \mathrm{u}[3]^{\wedge} 6^{*} \mathrm{y} 2{ }^{*} \mathrm{x} 3+64^{*} \mathrm{u}[3]^{\wedge} 6^{*} \mathrm{x} 2{ }^{*} \mathrm{y} 2+909^{*} \mathrm{u}[3]^{\wedge} 6^{*} \mathrm{x} 2{ }^{*} \mathrm{x} 3+ \\
& 312^{*} \mathrm{u}[3]^{\wedge} 6^{*} \mathrm{x} 1{ }^{*} \mathrm{x} 3-312^{*} \mathrm{u}[3]^{\wedge} 6^{*} \mathrm{x} 2{ }^{*} \mathrm{x} 0-329^{*} \mathrm{u}[3]^{\wedge} 4^{*} \mathrm{x} 2{ }^{*} \mathrm{x} 3+16^{*} \mathrm{u}[3]^{\wedge} 2^{*} \mathrm{y} 1{ }^{*} \mathrm{x} 0- \\
& 180^{*} \mathrm{u}[3]^{\wedge} 2^{*} \mathrm{x} 0^{*} \mathrm{y} 0+311^{*} \mathrm{u}[3]^{\wedge} 2^{*} \mathrm{x} 0^{*} \mathrm{x} 1+960^{*} \mathrm{u}[3]^{\wedge} 2^{*} \mathrm{x} 1^{*} \mathrm{y} 1+16^{*} \mathrm{u}[3]^{\wedge} 2^{*} \mathrm{y} 0^{*} \mathrm{x} 1+ \\
& 90{ }^{*} \mathrm{u}[3]^{\wedge} 6^{*} \mathrm{x} 0^{*} \mathrm{x} 1+966^{*} \mathrm{u}[3]^{\wedge} 6^{*} \mathrm{x} 0^{\wedge} 2+204^{*} \mathrm{u}[3]^{\wedge} 6^{*} \mathrm{x} 1^{\wedge} 2-542^{*} \mathrm{u}[3]^{\wedge} 2^{*} \mathrm{x} 0^{\wedge} 2+ \\
& 4052^{*} \mathrm{u}[3]^{\wedge} 2^{*} \mathrm{x} 1^{\wedge} 2+196^{*} \mathrm{x} 0^{*} \mathrm{y} 0+64^{*} \mathrm{x} 2^{*} \mathrm{y} 2-576^{*} \mathrm{u}[3]^{\wedge} 5^{*} \mathrm{x} 0^{\wedge} 2+576^{*} \mathrm{u}[3]^{\wedge} 5^{*} \mathrm{x} 1^{\wedge} 2- \\
& 4264^{*} \mathrm{u}[3]^{\wedge} 5^{*} \mathrm{x} 22^{*} \mathrm{x} 0+196^{*} \mathrm{x} 3^{*} \mathrm{y} 3-416^{*} \mathrm{u}[3]^{\wedge} 5^{*} \mathrm{y} 2^{*} \mathrm{x} 0+4264^{*} \mathrm{u}[3]^{\wedge} 5^{*} \mathrm{x} 1{ }^{*} \mathrm{x} 3- \\
& 480^{*} \mathrm{u}[3]^{\wedge} 5^{*} \mathrm{x} 0{ }^{*} \mathrm{x} 1+112^{*} \mathrm{y} 2{ }^{*} \mathrm{x} 3+416^{*} \mathrm{u}[3]^{\wedge} 5^{*} \mathrm{x} 1^{*} \mathrm{y} 3+196^{*} \mathrm{u}[3]^{\wedge} 6^{*} \mathrm{x} 0 * \mathrm{y} 0- \\
& 3848^{*} \mathrm{u}[3]^{*} \mathrm{x} 1^{*} \mathrm{x} 3+3848^{*} \mathrm{u}[3]^{*} \mathrm{x} 2^{*} \mathrm{x} 0+64^{*} \mathrm{u}[3]^{\wedge} 6^{*} \mathrm{x} 1^{*} \mathrm{y} 1+112^{*} \mathrm{u}[3]^{\wedge} 6^{*} \mathrm{y} 1^{*} \mathrm{x} 0+ \\
& 112^{*} \mathrm{u}[3]^{\wedge} 6^{*} \mathrm{y} 0^{*} \mathrm{x} 1+576^{*} \mathrm{u}[3]^{*} \mathrm{x} 3^{\wedge} 2-576^{*} \mathrm{u}[3]^{*} \mathrm{x} 2^{\wedge} 2+416^{*} \mathrm{u}[3]^{*} \mathrm{x} 2^{*} \mathrm{y} 0+480^{*} \mathrm{u}[3]^{*} \mathrm{x} 2^{*} \mathrm{x} 3- \\
& 416^{*} \mathrm{u}[3]^{*} \mathrm{x} 3^{*} \mathrm{y} 1+312^{*} \mathrm{x} 1{ }^{*} \mathrm{x} 3+204^{*} \mathrm{x} 22^{\wedge} 2+112^{*} \mathrm{y} 1^{*} \mathrm{x} 0+1248^{*} \mathrm{u}[3]^{\wedge} 3^{*} \mathrm{x} 2^{*} \mathrm{x} 0-
\end{aligned}
$$


$1248^{*} \mathrm{u}[3]^{\wedge} 3^{*} \mathrm{x} 1^{*} \mathrm{x} 3+204^{*} \mathrm{u}[3]^{\wedge} 6^{*} \mathrm{x} 2^{\wedge} 2+909^{*} \mathrm{x} 2^{*} \mathrm{x} 3+112^{*} \mathrm{x} 2^{*} \mathrm{y} 3+966^{*} \mathrm{u}[3]^{\wedge} 6^{*} \mathrm{x} 3^{\wedge} 2-$ $542^{*} \mathrm{u}[3]^{\wedge} 2^{*} \mathrm{x} 3^{\wedge} 2+4052^{*} \mathrm{u}[3]^{\wedge} 2^{*} \mathrm{x} 2 \wedge 2-312^{*} \mathrm{x} 2{ }^{*} \mathrm{x} 0-329^{*} \mathrm{u}[3]^{\wedge} 4^{*} \mathrm{x} 0{ }^{*} \mathrm{x} 1+16^{*} \mathrm{u}[3]^{\wedge} 4^{*} \mathrm{y} 1{ }^{*} \mathrm{x} 0-$ $180^{*} \mathrm{u}[3]^{\wedge} 4^{*} \mathrm{x} 0^{*} \mathrm{y} 0+1560^{*} \mathrm{u}[3]^{\wedge} 4^{*} \mathrm{x} 2^{*} \mathrm{x} 0-480^{*} \mathrm{u}[3]^{\wedge} 5^{*} \mathrm{x} 2^{*} \mathrm{x} 3+960^{*} \mathrm{u}[3]^{\wedge} 4^{*} \mathrm{x} 1^{*} \mathrm{y} 1+$ $16^{*} \mathrm{u}[3]^{\wedge} 4^{*} \mathrm{y} 0^{*} \mathrm{x} 1-1560^{*} \mathrm{u}[3]^{\wedge} 4^{*} \mathrm{x} 1^{*} \mathrm{x} 3-416^{*} \mathrm{u}[3]^{\wedge} 5^{*} \mathrm{x} 2^{*} \mathrm{y} 0-1310^{*} \mathrm{u}[3]^{\wedge} 4^{*} \mathrm{x} 0 \wedge 2+$ $4820^{*} \mathrm{u}[3]^{\wedge} 4^{*} \mathrm{x} 1^{\wedge} 2+112^{*} \mathrm{y} 0^{*} \mathrm{x} 1+909^{*} \mathrm{x} 0^{*} \mathrm{x} 1-576^{*} \mathrm{u}[3]^{\wedge} 5^{*} \mathrm{x} 3^{\wedge} 2-1310^{*} \mathrm{u}[3]^{\wedge} 4^{*} \mathrm{x} 3^{\wedge} 2+$ $416^{*} \mathrm{u}[3]^{\wedge} 5^{*} \mathrm{x} 3{ }^{*} \mathrm{y} 1+576^{*} \mathrm{u}[3]^{\wedge} 5^{*} \mathrm{x} 2^{\wedge} 2+4820^{*} \mathrm{u}[3]^{\wedge} 4^{*} \mathrm{x} 22^{\wedge} 2+64^{*} \mathrm{x} 1^{*} \mathrm{y} 1+966^{*} \mathrm{x} 0^{\wedge} 2+$ $966^{*} \mathrm{x} 3^{\wedge} 2+480^{*} \mathrm{u}[3]^{*} \mathrm{x} 0^{*} \mathrm{x} 1+416^{*} \mathrm{u}[3]^{*} \mathrm{y} 2^{*} \mathrm{x} 0+204^{*} \mathrm{x} 1^{\wedge} 2-416^{*} \mathrm{u}[3]^{*} \mathrm{x} 1^{*} \mathrm{y} 3+$ $576^{*} \mathrm{u}[3]^{*} \mathrm{x} 0^{\wedge} 2-180^{*} \mathrm{u}[3]^{\wedge} 4^{*} \mathrm{x} 3^{*} \mathrm{y} 3+16^{*} \mathrm{u}[3]^{\wedge} 4^{*} \mathrm{x} 2^{*} \mathrm{y} 3+16^{*} \mathrm{u}[3]^{\wedge} 4^{*} \mathrm{y} 2^{*} \mathrm{x} 3+$ $960 * \mathrm{u}[3]^{\wedge} 4^{*} \mathrm{x} 2 * \mathrm{y} 2-576^{*} \mathrm{u}[3]^{*} \mathrm{x} 1^{\wedge} 2$

$\mathrm{L} 3:=-96^{*} \mathrm{u}[3]^{\wedge} 2^{*} \mathrm{x} 2^{*} \mathrm{x} 3+112^{*} \mathrm{u}[3]^{\wedge} 4^{*} \mathrm{x} 2^{*} \mathrm{x} 3-96^{*} \mathrm{u}[3]^{\wedge} 2^{*} \mathrm{x} 0^{*} \mathrm{x} 1-214^{*} \mathrm{u}[3]^{\wedge} 2^{*} \mathrm{x} 0^{\wedge} 2+$ $422^{*} \mathrm{u}[3]^{\wedge} 2^{*} \mathrm{x} 1^{\wedge} 2-416^{*} \mathrm{u}[3]^{*} \mathrm{x} 1{ }^{*} \mathrm{x} 3+416^{*} \mathrm{u}[3]^{*} \mathrm{x} 2^{*} \mathrm{x} 0+19^{*} \mathrm{x} 22^{\wedge} 2-416^{*} \mathrm{u}[3]^{\wedge} 3^{*} \mathrm{x} 2^{*} \mathrm{x} 0+$ $416^{*} \mathrm{u}[3]^{\wedge} 3^{*} \mathrm{x} 1^{*} \mathrm{x} 3+112^{*} \mathrm{x} 2^{*} \mathrm{x} 3-214^{*} \mathrm{u}[3]^{\wedge} 2^{*} \mathrm{x} 3^{\wedge} 2+422^{*} \mathrm{u}[3]^{\wedge} 2^{*} \mathrm{x} 2^{\wedge} 2+112^{*} \mathrm{u}[3]^{\wedge} 4^{*} \mathrm{x} 0^{*} \mathrm{x} 1$ $+85^{*} \mathrm{u}[3]^{\wedge} 4^{*} \mathrm{x} 0^{\wedge} 2+19^{*} \mathrm{u}[3]^{\wedge} 4^{*} \mathrm{x} 1^{\wedge} 2+112^{*} \mathrm{x} 00^{*} \mathrm{x} 1+85^{*} \mathrm{u}[3]^{\wedge} 4^{*} \mathrm{x} 3^{\wedge} 2+19^{*} \mathrm{u}[3]^{\wedge} 4^{*} \mathrm{x} 2^{\wedge} 2+$ $85^{*} \mathrm{x} 00^{\wedge} 2+85^{*} \mathrm{x} 3^{\wedge} 2+19^{*} \mathrm{x} 1^{\wedge} 2$

$\mathrm{L} 4:=1345536^{*} \mathrm{u}[3]^{\wedge} 2^{*} \mathrm{x} 22^{*} \mathrm{y} 2+1252992^{*} \mathrm{u}[3]^{\wedge} 2^{*} \mathrm{x} 2^{*} \mathrm{x} 0+333056^{*} \mathrm{u}[3]^{\wedge} 2^{*} \mathrm{x} 2^{*} \mathrm{x} 3-$ $215424^{*} \mathrm{u}[3]^{\wedge} 6^{*} \mathrm{x} 3{ }^{*} \mathrm{y} 3+226944^{*} \mathrm{u}[3]^{\wedge} 2^{*} \mathrm{x} 3^{*} \mathrm{y} 3+119520^{*} \mathrm{u}[3]^{\wedge} 2^{*} \mathrm{y} 2^{*} \mathrm{x} 3-$ $1252992^{*} \mathrm{u}[3]^{\wedge} 2^{*} \mathrm{x} 1{ }^{*} \mathrm{x} 3+306720^{*} \mathrm{u}[3]^{\wedge} 2^{*} \mathrm{x} 22^{*} \mathrm{y} 3+122400 * \mathrm{u}[3]^{\wedge} 6^{*} \mathrm{x} 2 * \mathrm{y} 3-$ $64800^{*} \mathrm{u}[3]^{\wedge} 6^{*} \mathrm{y} 2^{*} \mathrm{x} 3+1787904^{*} \mathrm{u}[3]^{\wedge} 6^{*} \mathrm{x} 2^{*} \mathrm{y} 2-551680^{*} \mathrm{u}[3]^{\wedge} 6^{*} \mathrm{x} 2{ }^{*} \mathrm{x} 3-$ $2690688^{*} \mathrm{u}[3]^{\wedge} 6^{*} \mathrm{x} 11^{*} \mathrm{x} 3+2690688^{*} \mathrm{u}[3]^{\wedge} 6^{*} \mathrm{x} 2{ }^{*} \mathrm{x} 0+378608^{*} \mathrm{u}[3]^{\wedge} 4^{*} \mathrm{x} 2^{*} \mathrm{x} 3+$ $119520^{*} \mathrm{u}[3]^{\wedge} 2^{*} \mathrm{y} 1^{*} \mathrm{x} 0+226944^{*} \mathrm{u}[3]^{\wedge} 2^{*} \mathrm{x} 00^{*} \mathrm{y} 0+387488^{*} \mathrm{u}[3]^{\wedge} 2^{*} \mathrm{x} 0 * \mathrm{x} 1+$ $1345536^{*} \mathrm{u}[3]^{\wedge} 2^{*} \mathrm{x} 1^{*} \mathrm{y} 1+306720^{*} \mathrm{u}[3]^{\wedge} 2^{*} \mathrm{y} 0{ }^{*} \mathrm{x} 1-497248^{*} \mathrm{u}[3]^{\wedge} 6^{*} \mathrm{x} 0 * \mathrm{x} 1-$ $161468^{*} \mathrm{u}[3]^{\wedge} 6^{*} \mathrm{x} 0^{\wedge} 2+937828^{*} \mathrm{u}[3]^{\wedge} 6^{*} \mathrm{x} 1^{\wedge} 2+1211716^{*} \mathrm{u}[3]^{\wedge} 2^{*} \mathrm{x} 0^{\wedge} 2-435356^{*} \mathrm{u}[3]^{\wedge} 2^{*} \mathrm{x} 1^{\wedge} 2$ $+344736^{*} \mathrm{x} 0^{*} \mathrm{y} 0+48384^{*} \mathrm{x} 2^{*} \mathrm{y} 2-277968^{*} \mathrm{u}[3]^{\wedge} 5^{*} \mathrm{x} 0^{\wedge} 2+958128^{*} \mathrm{u}[3]^{\wedge} 5^{*} \mathrm{x} 1^{\wedge} 2+$ $22464^{*} \mathrm{u}[3]^{\wedge} 5^{*} \mathrm{x} 1{ }^{*} \mathrm{x} 2+2038192^{*} \mathrm{u}[3]^{\wedge} 5^{*} \mathrm{x} 2{ }^{*} \mathrm{x} 0+344736^{*} \mathrm{x} 3^{*} \mathrm{y} 3+475488^{*} \mathrm{u}[3]^{\wedge} 5^{*} \mathrm{y} 2^{*} \mathrm{x} 1-$ $232128^{*} \mathrm{u}[3]^{\wedge} 5^{*} \mathrm{y} 2 * \mathrm{x} 0-2038192^{*} \mathrm{u}[3]^{\wedge} 5^{*} \mathrm{x} 1{ }^{*} \mathrm{x} 3+22464^{*} \mathrm{u}[3]^{\wedge} 5^{*} \mathrm{x} 0 * \mathrm{x} 3-44544^{*} \mathrm{u}[3]^{\wedge} 5^{*} \mathrm{x} 0 * \mathrm{x} 1$ $+239616^{*} \mathrm{u}[3]^{\wedge} 5^{*} \mathrm{y} 1^{*} \mathrm{y} 3-331776^{*} \mathrm{u}[3]^{\wedge} 7^{*} \mathrm{x} 3^{*} \mathrm{y} 3-331776^{*} \mathrm{u}[3]^{\wedge} 7^{*} \mathrm{x} 0 * \mathrm{y} 0+$ $331776^{*} \mathrm{u}[3]^{\wedge} 7^{*} \mathrm{x} 22^{*} \mathrm{y} 2-238080^{*} \mathrm{u}[3]^{\wedge} 7^{*} \mathrm{y} 2^{*} \mathrm{x} 3+313040^{*} \mathrm{u}[3]^{\wedge} 7^{*} \mathrm{x} 1^{*} \mathrm{x} 3-$ $238080^{*} \mathrm{u}[3]^{\wedge} 7^{*} \mathrm{y} 1{ }^{*} \mathrm{x} 0-615936^{*} \mathrm{u}[3]^{\wedge} 7^{*} \mathrm{x} 2{ }^{*} \mathrm{x} 3-38400^{*} \mathrm{u}[3]^{\wedge} 7^{*} \mathrm{x} 2{ }^{*} \mathrm{y} 3+22464^{*} \mathrm{u}[3]^{\wedge} 7^{*} \mathrm{x} 1{ }^{*} \mathrm{x} 2$ $+22464^{*} \mathrm{u}[3]^{\wedge} 7^{*} \mathrm{x} 0^{*} \mathrm{x} 3-313040^{*} \mathrm{u}[3]^{\wedge} 7^{*} \mathrm{x} 2^{*} \mathrm{x} 0+313632^{*} \mathrm{u}[3]^{\wedge} 7^{*} \mathrm{y} 2^{*} \mathrm{x} 1-$ $38400^{*} \mathrm{u}[3]^{\wedge} 7^{*} \mathrm{y} 0^{*} \mathrm{x} 1-615936^{*} \mathrm{u}[3]^{\wedge} 7^{*} \mathrm{x} 00^{*} \mathrm{x} 1+331776^{*} \mathrm{u}[3]^{\wedge} 7^{*} \mathrm{x} 1{ }^{*} \mathrm{y} 1-313632^{*} \mathrm{u}[3]^{\wedge} 7^{*} \mathrm{y} 1{ }^{*} \mathrm{x} 2$ $-239616^{*} \mathrm{u}[3]^{\wedge} 7^{*} \mathrm{y} 0^{*} \mathrm{y} 2+239616^{*} \mathrm{u}[3]^{\wedge} 7^{*} \mathrm{y} 1^{*} \mathrm{y} 3+793152^{*} \mathrm{u}[3]^{\wedge} 7^{*} \mathrm{x} 1^{*} \mathrm{y} 3-$ $764352^{*} \mathrm{u}[3]^{\wedge} 7^{*} \mathrm{y} 2^{*} \mathrm{x} 0+764352^{*} \mathrm{u}[3]^{\wedge} 7^{*} \mathrm{x} 3^{*} \mathrm{y} 1-793152^{*} \mathrm{u}[3]^{\wedge} 7^{*} \mathrm{x} 2^{*} \mathrm{y} 0-$ $103968^{*} \mathrm{u}[3]^{\wedge} 7^{*} \mathrm{x} 00^{*} \mathrm{y} 3+103968^{*} \mathrm{u}[3]^{\wedge} 7^{*} \mathrm{x} 3^{*} \mathrm{y} 0+331776^{*} \mathrm{u}[3]^{*} \mathrm{x} 3{ }^{*} \mathrm{y} 3+331776^{*} \mathrm{u}[3]^{*} \mathrm{x} 0{ }^{*} \mathrm{y} 0-$ $331776^{*} \mathrm{u}[3]^{*} \mathrm{x} 2^{*} \mathrm{y} 2+238080^{*} \mathrm{u}[3]^{*} \mathrm{y} 2{ }^{*} \mathrm{x} 3+238080^{*} \mathrm{u}[3]^{*} \mathrm{y} 1^{*} \mathrm{x} 0+38400^{*} \mathrm{u}[3]^{*} \mathrm{x} 2{ }^{*} \mathrm{y} 3+$ $38400^{*} \mathrm{u}[3]^{*} \mathrm{y} 0{ }^{*} \mathrm{x} 1-331776^{*} \mathrm{u}[3]^{*} \mathrm{x} 1^{*} \mathrm{y} 1+239616^{*} \mathrm{u}[3]^{*} \mathrm{y} 00^{*} \mathrm{y} 2-239616^{*} \mathrm{u}[3]^{*} \mathrm{y} 1^{*} \mathrm{y} 3+$ $304992^{*} \mathrm{u}[3]^{\wedge} 4^{*} \mathrm{y} 2^{*} \mathrm{x} 1+18432^{*} \mathrm{u}[3]^{\wedge} 4^{*} \mathrm{y} 3^{*} \mathrm{y} 2-128640^{*} \mathrm{u}[3]^{\wedge} 4^{*} \mathrm{x} 1^{*} \mathrm{y} 3+$ $1668480^{*} \mathrm{u}[3]^{\wedge} 4^{*} \mathrm{y} 2{ }^{*} \mathrm{x} 0+204192^{*} \mathrm{u}[3]^{\wedge} 4^{*} \mathrm{x} 0 * \mathrm{y} 3+331776 * \mathrm{u}[3]^{\wedge} 3^{*} \mathrm{x} 33^{*} \mathrm{y} 3+$ $331776^{*} \mathrm{u}[3]^{\wedge} 3^{*} \mathrm{x} 00^{*} \mathrm{y} 0-331776^{*} \mathrm{u}[3]^{\wedge} 3^{*} \mathrm{x} 2{ }^{*} \mathrm{y} 2+211032^{*} \mathrm{y} 2{ }^{*} \mathrm{x} 3+606528^{*} \mathrm{u}[3]^{\wedge} 5^{*} \mathrm{x} 1{ }^{*} \mathrm{y} 3-$ $86112^{*} \mathrm{u}[3]^{\wedge} 5^{*} \mathrm{x} 0^{*} \mathrm{y} 3-215424^{*} \mathrm{u}[3]^{\wedge} 6^{*} \mathrm{x} 00^{*} \mathrm{y} 0+450736^{*} \mathrm{u}[3]^{*} \mathrm{x} 1^{*} \mathrm{x} 3-450736^{*} \mathrm{u}[3]^{*} \mathrm{x} 2{ }^{*} \mathrm{x} 0-$ $22464^{*} \mathrm{u}[3]^{*} \mathrm{x} 1{ }^{*} \mathrm{x} 2-22464^{*} \mathrm{u}[3]^{*} \mathrm{x} 00^{*} \mathrm{x} 3+1787904^{*} \mathrm{u}[3]^{\wedge} 6^{*} \mathrm{x} 1{ }^{*} \mathrm{y} 1-64800^{*} \mathrm{u}[3]^{\wedge} 6^{*} \mathrm{y} 1^{*} \mathrm{x} 0+$ $122400^{*} \mathrm{u}[3]^{\wedge} 6^{*} \mathrm{y} 0^{*} \mathrm{x} 1+1031184^{*} \mathrm{u}[3]^{*} \mathrm{x} 3^{\wedge} 2-737904^{*} \mathrm{u}[3]^{*} \mathrm{x} 2^{\wedge} 2+27792^{*} \mathrm{y} 2^{\wedge} 2+$ $500544^{*} \mathrm{u}[3]^{*} \mathrm{x} 2{ }^{*} \mathrm{y} 0+634368^{*} \mathrm{u}[3]^{*} \mathrm{x} 2{ }^{*} \mathrm{x} 3-817344^{*} \mathrm{u}[3]^{*} \mathrm{x} 3^{*} \mathrm{y} 1+151776^{*} \mathrm{u}[3]^{*} \mathrm{y} 1{ }^{*} \mathrm{x} 2-$ $121824^{*} \mathrm{u}[3]^{*} \mathrm{x} 3^{*} \mathrm{y} 0+535392^{*} \mathrm{x} 1{ }^{*} \mathrm{x} 3-331776^{*} \mathrm{u}[3]^{\wedge} 5^{*} \mathrm{x} 3^{*} \mathrm{y} 3-331776^{*} \mathrm{u}[3]^{\wedge} 5^{*} \mathrm{x} 00^{*} \mathrm{y} 0+$ $331776^{*} \mathrm{u}[3]^{\wedge} 5^{*} \mathrm{x} 2^{*} \mathrm{y} 2-238080^{*} \mathrm{u}[3]^{\wedge} 5^{*} \mathrm{y} 2^{*} \mathrm{x} 3-238080^{*} \mathrm{u}[3]^{\wedge} 5^{*} \mathrm{y} 1^{*} \mathrm{x} 0-38400^{*} \mathrm{u}[3]^{\wedge} 5^{*} \mathrm{x} 2^{*} \mathrm{y} 3$ 
$-38400^{*} \mathrm{u}[3]^{\wedge} 5^{*} \mathrm{y} 00^{*} \mathrm{x} 1+331776^{*} \mathrm{u}[3]^{\wedge} 5^{*} \mathrm{x} 1^{*} \mathrm{y} 1-239616^{*} \mathrm{u}[3]^{\wedge} 5^{*} \mathrm{y} 0^{*} \mathrm{y} 2+57565^{*} \mathrm{x} 2^{\wedge} 2-$ $22464^{*} \mathrm{u}[3]^{\wedge} 3^{*} \mathrm{x} 0{ }^{*} \mathrm{x} 3+211032^{*} \mathrm{y} 1^{*} \mathrm{x} 0-22464^{*} \mathrm{u}[3]^{\wedge} 3^{*} \mathrm{x} 1^{*} \mathrm{x} 2+1421264^{*} \mathrm{u}[3]^{\wedge} 3^{*} \mathrm{x} 2^{*} \mathrm{x} 0-$ $1421264^{*} \mathrm{u}[3]^{\wedge} 3^{*} \mathrm{x} 1^{*} \mathrm{x} 3+944740^{*} \mathrm{u}[3]^{\wedge} 6^{*} \mathrm{x} 2^{\wedge} 2+238080^{*} \mathrm{u}[3]^{\wedge} 3^{*} \mathrm{y} 2^{*} \mathrm{x} 3+$ $38400^{*} \mathrm{u}[3]^{\wedge} 3^{*} \mathrm{x} 2^{*} \mathrm{y} 3+10080^{*} \mathrm{u}[3]^{\wedge} 3^{*} \mathrm{y} 2^{*} \mathrm{x} 1+38400^{*} \mathrm{u}[3]^{\wedge} 3^{*} \mathrm{y} 0^{*} \mathrm{x} 1+$ $1205760^{*} \mathrm{u}[3]^{\wedge} 3^{*} \mathrm{x} 0{ }^{*} \mathrm{x} 1-331776^{*} \mathrm{u}[3]^{\wedge} 3^{*} \mathrm{x} 1{ }^{*} \mathrm{y} 1-10080^{*} \mathrm{u}[3]^{\wedge} 3^{*} \mathrm{y} 1{ }^{*} \mathrm{x} 2+$ $239616^{*} \mathrm{u}[3]^{\wedge} 3^{*} \mathrm{y} 0^{*} \mathrm{y} 2-239616^{*} \mathrm{u}[3]^{\wedge} 3^{*} \mathrm{y} 1^{*} \mathrm{y} 3-687168^{*} \mathrm{u}[3]^{\wedge} 3^{*} \mathrm{x} 1^{*} \mathrm{y} 3+$ $1349568^{*} \mathrm{u}[3]^{\wedge} 3^{*} \mathrm{y} 2^{*} \mathrm{x} 0-1349568^{*} \mathrm{u}[3]^{\wedge} 3^{*} \mathrm{x} 3^{*} \mathrm{y} 1+687168^{*} \mathrm{u}[3]^{\wedge} 3^{*} \mathrm{x} 2{ }^{*} \mathrm{y} 0+$ $139680^{*} \mathrm{u}[3]^{\wedge} 3^{*} \mathrm{x} 00^{*} \mathrm{y} 3-139680^{*} \mathrm{u}[3]^{\wedge} 3^{*} \mathrm{x} 3^{*} \mathrm{y} 0+344736^{*} \mathrm{u}[3]^{\wedge} 8^{*} \mathrm{x} 3^{*} \mathrm{y} 3+$ $211032^{*} \mathrm{u}[3]^{\wedge} 8^{*} \mathrm{y} 2{ }^{*} \mathrm{x} 3+48384^{*} \mathrm{u}[3]^{\wedge} 8^{*} \mathrm{x} 2{ }^{*} \mathrm{y} 2-206456^{*} \mathrm{u}[3]^{\wedge} 8^{*} \mathrm{x} 2{ }^{*} \mathrm{x} 3+70632^{*} \mathrm{u}[3]^{\wedge} 8^{*} \mathrm{x} 2{ }^{*} \mathrm{y} 3$ $+64512^{*} \mathrm{u}[3]^{\wedge} 8^{*} \mathrm{y} 3^{*} \mathrm{y} 2-213584^{*} \mathrm{u}[3]^{\wedge} 8^{*} \mathrm{x} 0 * \mathrm{x} 1+192576^{*} \mathrm{u}[3]^{\wedge} 8^{*} \mathrm{x} 1^{*} \mathrm{y} 3+$ $12864^{*} \mathrm{u}[3]^{\wedge} 8^{*} \mathrm{y} 2{ }^{*} \mathrm{x} 0-206456^{*} \mathrm{x} 2 * \mathrm{x} 3+70632^{*} \mathrm{x} 2{ }^{*} \mathrm{y} 3-168380^{*} \mathrm{u}[3]^{\wedge} 6^{*} \mathrm{x} 3{ }^{\wedge} 2+$ $1204804^{*} \mathrm{u}[3]^{\wedge} 2^{*} \mathrm{x} 3^{\wedge} 2-428444^{*} \mathrm{u}[3]^{\wedge} 2^{*} \mathrm{x} 2^{\wedge} 2-27408^{*} \mathrm{u}[3]^{\wedge} 8^{*} \mathrm{x} 00^{*} \mathrm{y} 3+112272^{*} \mathrm{u}[3]^{\wedge} 8^{*} \mathrm{y} 2{ }^{*} \mathrm{x} 1-$ $535392^{*} \mathrm{x} 2{ }^{*} \mathrm{x} 0+112272^{*} \mathrm{y} 2^{*} \mathrm{x} 1+64512^{*} \mathrm{y} 3^{*} \mathrm{y} 2+501728^{*} \mathrm{u}[3]^{\wedge} 4^{*} \mathrm{x} 0 * \mathrm{x} 1-$ $367344^{*} \mathrm{u}[3]^{\wedge} 4^{*} \mathrm{y} 1^{*} \mathrm{x} 0-677952^{*} \mathrm{u}[3]^{\wedge} 4^{*} \mathrm{x} 0 * \mathrm{y} 0+5014464^{*} \mathrm{u}[3]^{\wedge} 4^{*} \mathrm{x} 2^{*} \mathrm{x} 0-$ $44544^{*} \mathrm{u}[3]^{\wedge} 5^{*} \mathrm{x} 2{ }^{*} \mathrm{x} 3+3036672^{*} \mathrm{u}[3]^{\wedge} 4^{*} \mathrm{x} 1^{*} \mathrm{y} 1+287856^{*} \mathrm{u}[3]^{\wedge} 4^{*} \mathrm{y} 0^{*} \mathrm{x} 1-$ $5014464^{*} \mathrm{u}[3]^{\wedge} 4^{*} \mathrm{x} 1^{*} \mathrm{x} 3-606528^{*} \mathrm{u}[3]^{\wedge} 5^{*} \mathrm{x} 2^{*} \mathrm{y} 0+42048^{*} \mathrm{u}[3]^{\wedge} 6^{*} \mathrm{y} 3^{\wedge} 2+332352^{*} \mathrm{u}[3]^{\wedge} 6^{*} \mathrm{y} 2^{\wedge} 2$ $-475488^{*} \mathrm{u}[3]^{\wedge} 5^{*} \mathrm{y} 1^{*} \mathrm{x} 2+939486^{*} \mathrm{u}[3]^{\wedge} 4^{*} \mathrm{x} 0^{\wedge} 2+178254^{*} \mathrm{u}[3]^{\wedge} 4^{*} \mathrm{x} 1^{\wedge} 2+$ $86112^{*} \mathrm{u}[3]^{\wedge} 5^{*} \mathrm{x} 3^{*} \mathrm{y} 0+70632^{*} \mathrm{y} 0{ }^{*} \mathrm{x} 1-213584^{*} \mathrm{x} 0^{*} \mathrm{x} 1-277968^{*} \mathrm{u}[3]^{\wedge} 5^{*} \mathrm{x} 3^{\wedge} 2+$ $937758^{*} \mathrm{u}[3]^{\wedge} 4^{*} \mathrm{x} 3^{\wedge} 2+232128^{*} \mathrm{u}[3]^{\wedge} 5^{*} \mathrm{x} 3^{*} \mathrm{y} 1+958128^{*} \mathrm{u}[3]^{\wedge} 5^{*} \mathrm{x} 2^{\wedge} 2+179982^{*} \mathrm{u}[3]^{\wedge} 4^{*} \mathrm{x} 2^{\wedge} 2$ $+1179792^{*} \mathrm{u}[3]^{\wedge} 7^{*} \mathrm{x} 2^{\wedge} 2-986352^{*} \mathrm{u}[3]^{\wedge} 7^{*} \mathrm{x} 0^{\wedge} 2-986352^{*} \mathrm{u}[3]^{\wedge} 7^{*} \mathrm{x} 3^{\wedge} 2+$ $1179792^{*} \mathrm{u}[3]^{\wedge} 7^{*} \mathrm{x} 1^{\wedge} 2+65808^{*} \mathrm{y} 3^{\wedge} 2+609120^{*} \mathrm{u}[3]^{\wedge} 4^{*} \mathrm{y} 1^{\wedge} 2-47520^{*} \mathrm{u}[3]^{\wedge} 4^{*} \mathrm{y} 0^{\wedge} 2+$ $48384^{*} \mathrm{x} 1^{*} \mathrm{y} 1+165973^{*} \mathrm{x} 0^{\wedge} 2+159925^{*} \mathrm{x} 3^{\wedge} 2-112272^{*} \mathrm{y} 1^{*} \mathrm{x} 2+634368^{*} \mathrm{u}[3]^{*} \mathrm{x} 0{ }^{*} \mathrm{x} 1+$ $817344^{*} \mathrm{u}[3]^{*} \mathrm{y} 2^{*} \mathrm{x} 0+51517^{*} \mathrm{x} 1^{\star} 2-500544^{*} \mathrm{u}[3]^{*} \mathrm{x} 1^{*} \mathrm{y} 3+121824^{*} \mathrm{u}[3]^{*} \mathrm{x} 00^{*} \mathrm{y} 3-$

$151776^{*} \mathrm{u}[3]^{*} \mathrm{y} 2{ }^{*} \mathrm{x} 1+1031184^{*} \mathrm{u}[3]^{*} \mathrm{x} 0{ }^{\wedge} 2-677952^{*} \mathrm{u}[3]^{\wedge} 4^{*} \mathrm{x} 3{ }^{*} \mathrm{y} 3+287856^{*} \mathrm{u}[3]^{\wedge} 4^{*} \mathrm{x} 2{ }^{*} \mathrm{y} 3-$ $367344^{*} \mathrm{u}[3]^{\wedge} 4^{*} \mathrm{y} 2{ }^{*} \mathrm{x} 3+3036672^{*} \mathrm{u}[3]^{\wedge} 4^{*} \mathrm{x} 2^{*} \mathrm{y} 2+42048^{*} \mathrm{u}[3]^{\wedge} 2^{*} \mathrm{y} 3^{\wedge} 2+$ $73728^{*} \mathrm{u}[3]^{\wedge} 2^{*} \mathrm{y} 3{ }^{*} \mathrm{y} 2+128256^{*} \mathrm{u}[3]^{\wedge} 2^{*} \mathrm{x} 1{ }^{*} \mathrm{y} 3+847104 * \mathrm{u}[3]^{\wedge} 2{ }^{*} \mathrm{y} 2{ }^{*} \mathrm{x} 0+$ $609120^{*} \mathrm{u}[3]^{\wedge} 4^{*} \mathrm{y} 2^{\wedge} 2-47520^{*} \mathrm{u}[3]^{\wedge} 4^{*} \mathrm{y} 3^{\wedge} 2+238080^{*} \mathrm{u}[3]^{\wedge} 3^{*} \mathrm{y} 1^{*} \mathrm{x} 0+1205760^{*} \mathrm{u}[3]^{\wedge} 3^{*} \mathrm{x} 2^{*} \mathrm{x} 3$ $-959568^{*} \mathrm{u}[3]^{\wedge} 3^{*} \mathrm{x} 2^{\wedge} 2+27792^{*} \mathrm{y} 1^{\wedge} 2+65808^{*} \mathrm{y} 0^{\wedge} 2-737904^{*} \mathrm{u}[3]^{*} \mathrm{x} 1^{\wedge} 2+64512^{*} \mathrm{y} 0 * \mathrm{y} 1+$ $192576^{*} \mathrm{x} 1{ }^{*} \mathrm{y} 3+12864 * \mathrm{y} 2{ }^{*} \mathrm{x} 0-12864^{*} \mathrm{x} 3{ }^{*} \mathrm{y} 1-192576^{*} \mathrm{x} 2{ }^{*} \mathrm{y} 0-27408^{*} \mathrm{x} 0 * \mathrm{y} 3+$ $27408^{*} \mathrm{x} 3^{*} \mathrm{y} 0+73728^{*} \mathrm{u}[3]^{\wedge} 2^{*} \mathrm{y} 0 * \mathrm{y} 1-847104^{*} \mathrm{u}[3]^{\wedge} 2^{*} \mathrm{x} 3^{*} \mathrm{y} 1+332352^{*} \mathrm{u}[3]^{\wedge} 2^{*} \mathrm{y} 1^{\wedge} 2+$ $42048^{*} \mathrm{u}[3]^{\wedge} 2^{*} \mathrm{y} 0 \wedge \wedge+332352^{*} \mathrm{u}[3]^{\wedge} 6^{*} \mathrm{y} 1^{\wedge} 2+42048^{*} \mathrm{u}[3]^{\wedge} 6^{*} \mathrm{y} 0 \wedge \wedge+1739568^{*} \mathrm{u}[3]^{\wedge} 3^{*} \mathrm{x} 00^{\wedge} 2+$ $1739568^{*} \mathrm{u}[3]^{\wedge} 3^{*} \mathrm{x} 3^{\wedge} 2-959568^{*} \mathrm{u}[3]^{\wedge} 3^{*} \mathrm{x} 1^{\wedge} 2+27792^{*} \mathrm{u}[3]^{\wedge} 8^{*} \mathrm{y} 2^{\wedge} 2+57565^{*} \mathrm{u}[3]^{\wedge} 8^{*} \mathrm{x} 2^{\wedge} 2+$ $65808^{*} \mathrm{u}[3]^{\wedge} 8^{*} \mathrm{y} 3^{\wedge} 2+165973^{*} \mathrm{u}[3]^{\wedge} 8^{*} \mathrm{x} 0^{\wedge} 2+159925^{*} \mathrm{u}[3]^{\wedge} 8^{*} \mathrm{x} 3^{\wedge} 2+51517^{*} \mathrm{u}[3]^{\wedge} 8^{*} \mathrm{x} 1^{\wedge} 2+$ $332352^{*} \mathrm{u}[3]^{\wedge} 2^{*} \mathrm{y} 22^{\wedge} 2-128256^{*} \mathrm{u}[3]^{\wedge} 2^{*} \mathrm{x} 2^{*} \mathrm{y} 0+73728^{*} \mathrm{u}[3]^{\wedge} 6^{*} \mathrm{y} 0^{*} \mathrm{y} 1+64512^{*} \mathrm{u}[3]^{\wedge} 8^{*} \mathrm{y} 0 * \mathrm{y} 1$ $+18432^{*} \mathrm{u}[3]^{\wedge} 4^{*} \mathrm{y} 0^{*} \mathrm{y} 1+73728^{*} \mathrm{u}[3]^{\wedge} 6^{*} \mathrm{y} 3^{*} \mathrm{y} 2+128256^{*} \mathrm{u}[3]^{\wedge} 6^{*} \mathrm{x} 11^{*} \mathrm{y} 3+$ $847104^{*} \mathrm{u}[3]^{\wedge} 6^{*} \mathrm{y} 2 * \mathrm{x} 0-535392^{*} \mathrm{u}[3]^{\wedge} 8^{*} \mathrm{x} 2{ }^{*} \mathrm{x} 0+535392^{*} \mathrm{u}[3]^{\wedge} 8^{*} \mathrm{x} 1{ }^{*} \mathrm{x} 3-$ $192576^{*} \mathrm{u}[3]^{\wedge} 8^{*} \mathrm{x} 2^{*} \mathrm{y} 0+27408^{*} \mathrm{u}[3]^{\wedge} 8^{*} \mathrm{x} 3^{*} \mathrm{y} 0-112272^{*} \mathrm{u}[3]^{\wedge} 8^{*} \mathrm{y} 1^{*} \mathrm{x} 2-12864^{*} \mathrm{u}[3]^{\wedge} 8^{*} \mathrm{x} 3^{*} \mathrm{y} 1$ $+128640^{*} \mathrm{u}[3]^{\wedge} 4^{*} \mathrm{x} 2^{*} \mathrm{y} 0-204192^{*} \mathrm{u}[3]^{\wedge} 4^{*} \mathrm{x} 3^{*} \mathrm{y} 0-304992^{*} \mathrm{u}[3]^{\wedge} 4^{*} \mathrm{y} 1^{*} \mathrm{x} 2-$ $1668480^{*} \mathrm{u}[3]^{\wedge} 4^{*} \mathrm{x} 3^{*} \mathrm{y} 1+344736^{*} \mathrm{u}[3]^{\wedge} 8^{*} \mathrm{x} 0^{*} \mathrm{y} 0+211032^{*} \mathrm{u}[3]^{\wedge} 8^{*} \mathrm{y} 1^{*} \mathrm{x} 0+$ $70632^{*} \mathrm{u}[3]^{\wedge} 8^{*} \mathrm{y} 0^{*} \mathrm{x} 1+48384^{*} \mathrm{u}[3]^{\wedge} 8^{*} \mathrm{x} 1^{*} \mathrm{y} 1-128256^{*} \mathrm{u}[3]^{\wedge} 6^{*} \mathrm{x} 2^{*} \mathrm{y} 0-264768^{*} \mathrm{u}[3]^{\wedge} 6^{*} \mathrm{y} 1^{*} \mathrm{x} 2$ $-74688^{*} \mathrm{u}[3]^{\wedge} 6^{*} \mathrm{x} 3^{*} \mathrm{y} 0-847104^{*} \mathrm{u}[3]^{\wedge} 6^{*} \mathrm{x} 3^{*} \mathrm{y} 1+264768^{*} \mathrm{u}[3]^{\wedge} 2^{*} \mathrm{y} 2^{*} \mathrm{x} 1+$ $74688^{*} \mathrm{u}[3]^{\wedge} 2^{*} \mathrm{x} 00^{*} \mathrm{y} 3+264768^{*} \mathrm{u}[3]^{\wedge} 6^{*} \mathrm{y} 2^{*} \mathrm{x} 1+74688^{*} \mathrm{u}[3]^{\wedge} 6^{*} \mathrm{x} 00^{*} \mathrm{y} 3+27792^{*} \mathrm{u}[3]^{\wedge} 8^{*} \mathrm{y} 1^{\wedge} 2$ $+65808^{*} \mathrm{u}[3]^{\wedge} 8^{*} \mathrm{y} 0 \wedge 2-264768^{*} \mathrm{u}[3]^{\wedge} 2^{*} \mathrm{y} 1^{*} \mathrm{x} 2-74688^{*} \mathrm{u}[3]^{\wedge} 2^{*} \mathrm{x} 3^{*} \mathrm{y} 0$

$\mathrm{L} 5:=128144^{*} \mathrm{u}[3]^{*} \mathrm{x} 2 \wedge 3+1152^{*} \mathrm{y} 1^{*} \mathrm{x} 2 \wedge 2-76904^{*} \mathrm{u}[3]^{\wedge} 6^{*} \mathrm{x} 0 \wedge 3-67840^{*} \mathrm{u}[3]^{\wedge} 6^{*} \mathrm{x} 1^{\wedge} 3+$ $8960^{*} \mathrm{u}[3]^{\wedge} 8^{*} \mathrm{x} 0^{\wedge} 3-5120^{*} \mathrm{u}[3]^{\wedge} 8^{*} \mathrm{x} 1^{\wedge} 3-538752^{*} \mathrm{u}[3]^{\wedge} 6^{*} \mathrm{y} 2^{*} \mathrm{x} 0^{*} \mathrm{x} 1+$ 
$161040^{*} \mathrm{u}[3]^{\wedge} 6^{*} \mathrm{y} 3{ }^{*} \mathrm{x} 1{ }^{*} \mathrm{x} 0+10986^{*} \mathrm{u}[3]^{\wedge} 8^{*} \mathrm{x} 1{ }^{*} \mathrm{x} 2{ }^{*} \mathrm{x} 0-149352^{*} \mathrm{u}[3]^{\wedge} 8^{*} \mathrm{x} 3{ }^{*} \mathrm{x} 0{ }^{*} \mathrm{x} 1-$ $99840^{*} \mathrm{u}[3]^{\wedge} 8^{*} \mathrm{y} 2{ }^{*} \mathrm{x} 0{ }^{*} \mathrm{x} 1-7800^{*} \mathrm{u}[3]^{\wedge} 8^{*} \mathrm{y} 3^{*} \mathrm{x} 1{ }^{*} \mathrm{x} 0-59904^{*} \mathrm{u}[3]^{\wedge} 5^{*} \mathrm{y} 3{ }^{*} \mathrm{y} 2{ }^{*} \mathrm{x} 3+$ $43200^{*} \mathrm{u}[3]^{\wedge} 5^{*} \mathrm{y} 3^{*} \mathrm{x} 00^{*} \mathrm{x} 3-768^{*} \mathrm{u}[3]^{\wedge} 5^{*} \mathrm{y} 3^{*} \mathrm{x} 1{ }^{*} \mathrm{x} 2+36096^{*} \mathrm{u}[3]^{\wedge} 5^{*} \mathrm{x} 3^{*} \mathrm{y} 3^{*} \mathrm{x} 1+$ $702720^{*} \mathrm{u}[3]^{\wedge} 5^{*} \mathrm{y} 2^{*} \mathrm{x} 2{ }^{*} \mathrm{x} 1-287808^{*} \mathrm{u}[3]^{\wedge} 5^{*} \mathrm{y} 2^{*} \mathrm{x} 1^{*} \mathrm{x} 3+81600^{*} \mathrm{u}[3]^{\wedge} 5^{*} \mathrm{x} 2^{*} \mathrm{y} 2^{*} \mathrm{x} 0-$ $331440^{*} \mathrm{u}[3]^{\wedge} 5^{*} \mathrm{x} 0{ }^{*} \mathrm{y} 3{ }^{*} \mathrm{x} 2+155904 * \mathrm{u}[3]^{\wedge} 5^{*} \mathrm{x} 3{ }^{*} \mathrm{y} 2{ }^{*} \mathrm{x} 0+104832^{*} \mathrm{u}[3]^{\wedge} 7^{*} \mathrm{y} 3{ }^{*} \mathrm{y} 2{ }^{*} \mathrm{x} 2+$ $59904^{*} \mathrm{u}[3]^{\wedge} 7^{*} \mathrm{y} 3^{*} \mathrm{y} 2^{*} \mathrm{x} 3-47040^{*} \mathrm{u}[3]^{\wedge} 7^{*} \mathrm{y} 3^{*} \mathrm{x} 0{ }^{*} \mathrm{x} 3+94464^{*} \mathrm{u}[3]^{\wedge} 7^{*} \mathrm{y} 3^{*} \mathrm{x} 1^{*} \mathrm{x} 2+$ $33336^{*} \mathrm{u}[3]^{*} \mathrm{x} 3^{\wedge} 2^{*} \mathrm{x} 2+688946^{*} \mathrm{u}[3]^{\wedge} 3^{*} \mathrm{x} 0^{\wedge} 2^{*} \mathrm{x} 2+79536^{*} \mathrm{u}[3]^{\wedge} 3^{*} \mathrm{x} 1^{\wedge} 2^{*} \mathrm{x} 2-$ $118908^{*} \mathrm{u}[3]^{\wedge} 3^{*} \mathrm{x} 0^{\wedge} 2^{*} \mathrm{x} 3+28224^{*} \mathrm{y} 2^{\wedge} 2^{*} \mathrm{x} 0-28224^{*} \mathrm{y} 3^{\wedge} 2^{*} \mathrm{x} 0+16128^{*} \mathrm{y} 2^{\wedge} 2^{*} \mathrm{x} 1-$ $16128^{*} \mathrm{y} 3^{\wedge} 2^{*} \mathrm{x} 1+43008^{*} \mathrm{x} 22^{\wedge} 2^{*} \mathrm{y} 2-5880^{*} \mathrm{x} 3^{\wedge} 2^{*} \mathrm{y} 2+3360^{*} \mathrm{x} 3{ }^{\wedge} 2^{*} \mathrm{y} 3-3072^{*} \mathrm{x} 1{ }^{\wedge} 2^{*} \mathrm{x} 2+$ $37128^{*} \mathrm{x} 0{ }^{\wedge} 2^{*} \mathrm{y} 2+50394^{*} \mathrm{x} 1{ }^{\wedge} 2^{*} \mathrm{x} 3+74892^{*} \mathrm{x} 33^{\wedge} 2^{*} \mathrm{x} 2-54144^{*} \mathrm{u}[3]^{\wedge} 6^{*} \mathrm{y} 3^{\wedge} 2^{*} \mathrm{x} 0-$ $225792^{*} \mathrm{u}[3]^{\wedge} 6^{*} \mathrm{y} 2^{\wedge} 2^{*} \mathrm{x} 1+24192^{*} \mathrm{u}[3]^{\wedge} 6^{*} \mathrm{y} 2^{\wedge} 2^{*} \mathrm{x} 0-13824^{*} \mathrm{u}[3]^{\wedge} 6^{*} \mathrm{y} 3^{\wedge} 2^{*} \mathrm{x} 1+$ $402600^{*} \mathrm{u}[3]^{\wedge} 3^{*} \mathrm{x} 0{ }^{\wedge} 2^{*} \mathrm{x} 1-397524^{*} \mathrm{u}[3]^{\wedge} 3^{*} \mathrm{x} 3{ }^{*} \mathrm{x} 2{ }^{*} \mathrm{x} 1+89316^{*} \mathrm{u}[3]^{*} \mathrm{x} 3{ }^{*} \mathrm{x} 2{ }^{*} \mathrm{x} 1+$ $233184^{*} \mathrm{u}[3]^{*} \mathrm{x} 1^{*} \mathrm{x} 2{ }^{*} \mathrm{x} 0+249186^{*} \mathrm{u}[3]^{*} \mathrm{x} 3^{*} \mathrm{x} 00^{*} \mathrm{x} 1-472514^{*} \mathrm{u}[3]^{\wedge} 6^{*} \mathrm{x} 2^{*} \mathrm{x} 0^{*} \mathrm{x} 3-$ $118324^{*} \mathrm{u}[3]^{\wedge} 6^{*} \mathrm{x} 3^{*} \mathrm{x} 2^{*} \mathrm{x} 1-107856^{*} \mathrm{u}[3]^{\wedge} 6^{*} \mathrm{y} 0^{*} \mathrm{x} 3^{*} \mathrm{x} 2+200448^{*} \mathrm{u}[3]^{\wedge} 6^{*} \mathrm{y} 1^{*} \mathrm{x} 2^{*} \mathrm{x} 3+$ $127764^{*} \mathrm{u}[3]^{\wedge} 6^{*} \mathrm{x} 1^{*} \mathrm{x} 2{ }^{*} \mathrm{x} 0-41808^{*} \mathrm{u}[3]^{\wedge} 6^{*} \mathrm{x} 3^{*} \mathrm{x} 0{ }^{*} \mathrm{x} 1+302592^{*} \mathrm{u}[3]^{*} \mathrm{x} 2{ }^{*} \mathrm{x} 0{ }^{*} \mathrm{x} 3+$ $2016^{*} \mathrm{y} 0^{*} \mathrm{x} 2^{\wedge} 2-27360^{*} \mathrm{u}[3]^{\wedge} 7^{*} \mathrm{y} 2^{*} \mathrm{x} 0^{*} \mathrm{x} 1-24480^{*} \mathrm{u}[3]^{\wedge} 7^{*} \mathrm{y} 3^{*} \mathrm{x} 1^{*} \mathrm{x} 0-$ $101664^{*} \mathrm{u}[3]^{\wedge} 5^{*} \mathrm{y} 2^{*} \mathrm{x} 00^{*} \mathrm{x} 1+139392^{*} \mathrm{u}[3]^{\wedge} 5^{*} \mathrm{y} 3^{*} \mathrm{x} 1^{*} \mathrm{x} 0+41688^{*} \mathrm{u}[3]^{\wedge} 6^{*} \mathrm{y} 3^{*} \mathrm{x} 0{ }^{*} \mathrm{x} 3+$ $114624^{*} \mathrm{u}[3]^{\wedge} 6^{*} \mathrm{y} 3^{*} \mathrm{x} 1{ }^{*} \mathrm{x} 2-100800^{*} \mathrm{u}[3]^{\wedge} 6^{*} \mathrm{x} 3^{*} \mathrm{y} 3^{*} \mathrm{x} 1+48384^{*} \mathrm{u}[3]^{\wedge} 6^{*} \mathrm{y} 2^{*} \mathrm{x} 2^{*} \mathrm{x} 1-$ $148392^{*} \mathrm{u}[3]^{\wedge} 6^{*} \mathrm{y} 2{ }^{*} \mathrm{x} 1{ }^{*} \mathrm{x} 3+80928^{*} \mathrm{u}[3]^{\wedge} 6^{*} \mathrm{y} 3{ }^{*} \mathrm{y} 2{ }^{*} \mathrm{x} 0-109080^{*} \mathrm{u}[3]^{\wedge} 6^{*} \mathrm{x} 2{ }^{*} \mathrm{y} 2{ }^{*} \mathrm{x} 0+$ $193104^{*} \mathrm{u}[3]^{\wedge} 6^{*} \mathrm{x} 0 * \mathrm{y} 3^{*} \mathrm{x} 2-14688^{*} \mathrm{u}[3]^{\wedge} 6^{*} \mathrm{x} 3^{*} \mathrm{y} 2^{*} \mathrm{x} 0+153216^{*} \mathrm{u}[3]^{\wedge} 6^{*} \mathrm{y} 3^{*} \mathrm{y} 2^{*} \mathrm{x} 1-$ $239616^{*} \mathrm{u}[3]^{\wedge} 6^{*} \mathrm{y} 0{ }^{*} \mathrm{y} 1{ }^{*} \mathrm{x} 0-29952^{*} \mathrm{u}[3]^{\wedge} 6^{*} \mathrm{y} 0{ }^{*} \mathrm{y} 1^{*} \mathrm{x} 1-114624^{*} \mathrm{u}[3]^{\wedge} 4^{*} \mathrm{y} 0{ }^{*} \mathrm{x} 3^{*} \mathrm{x} 2+$ $340992^{*} \mathrm{u}[3]^{\wedge} 4^{*} \mathrm{y} 1{ }^{*} \mathrm{x} 2{ }^{*} \mathrm{x} 3-104832^{*} \mathrm{u}[3]^{\wedge} 5^{*} \mathrm{y} 3{ }^{*} \mathrm{y} 2{ }^{*} \mathrm{x} 2+104832^{*} \mathrm{u}[3]^{\wedge} 5^{*} \mathrm{y} 22^{\wedge} 2^{*} \mathrm{x} 3+$ $59904^{*} \mathrm{u}[3]^{\wedge} 5^{*} \mathrm{y} 3^{\wedge} 2^{*} \mathrm{x} 2+230400^{*} \mathrm{u}[3]^{\wedge} 4^{*} \mathrm{x} 2^{\wedge} 3-73728^{*} \mathrm{u}[3]^{\wedge} 4^{*} \mathrm{x} 3^{\wedge} 3-$

$253440^{*} \mathrm{u}[3]^{\wedge} 2^{*} \mathrm{y} 0{ }^{*} \mathrm{y} 3^{*} \mathrm{x} 2-225792^{*} \mathrm{u}[3]^{\wedge} 2^{*} \mathrm{y} 1^{*} \mathrm{y} 2^{*} \mathrm{x} 2-106944^{*} \mathrm{u}[3]^{\wedge} 2^{*} \mathrm{x} 2^{*} \mathrm{y} 2^{*} \mathrm{x} 3-$

$212736^{*} \mathrm{u}[3]^{\wedge} 2^{*} \mathrm{x} 2{ }^{*} \mathrm{y} 3{ }^{*} \mathrm{x} 3+158976^{*} \mathrm{u}[3]^{\wedge} 2^{*} \mathrm{y} 1{ }^{*} \mathrm{y} 3^{*} \mathrm{x} 2+51156^{*} \mathrm{y} 3{ }^{*} \mathrm{x} 0 * \mathrm{x} 3-14688^{*} \mathrm{y} 1{ }^{*} \mathrm{x} 2{ }^{*} \mathrm{x} 3$ $-2016^{*} \mathrm{y} 3^{*} \mathrm{x} 1{ }^{*} \mathrm{x} 2+50016^{*} \mathrm{u}[3]^{\wedge} 7^{*} \mathrm{x} 22^{\wedge} 2^{*} \mathrm{x} 3-90936^{*} \mathrm{u}[3]^{\wedge} 7^{*} \mathrm{x} 3^{\wedge} 2^{*} \mathrm{x} 2-$

$118944^{*} \mathrm{u}[3]^{\wedge} 7^{*} \mathrm{x} 22^{\wedge} 2^{*} \mathrm{y} 2-81360^{*} \mathrm{u}[3]^{\wedge} 7^{*} \mathrm{x} 3^{\wedge} 2^{*} \mathrm{y} 2+60480^{*} \mathrm{u}[3]^{\wedge} 7^{*} \mathrm{x} 22^{\wedge} 2^{*} \mathrm{y} 3-$ $15552 * \mathrm{u}[3]^{\wedge} 7{ }^{*} \mathrm{x} 3{ }^{\wedge} 2{ }^{*} \mathrm{y} 3+338136 * \mathrm{u}[3]{ }^{*} \mathrm{x} 0{ }^{\wedge} 2{ }^{*} \mathrm{x} 1+103824 * \mathrm{u}[3]{ }^{*} \mathrm{x} 0{ }^{\wedge} 2{ }^{*} \mathrm{y} 2+$ $85752^{*} \mathrm{u}[3]^{*} \mathrm{x} 0{ }^{\wedge} 2^{*} \mathrm{y} 3-36144^{*} \mathrm{u}[3]^{*} \mathrm{x} 1^{\wedge} 2^{*} \mathrm{x} 0-68256^{*} \mathrm{u}[3]^{*} \mathrm{y} 2^{*} \mathrm{x} 1^{\wedge} 2+6912^{*} \mathrm{u}[3]^{*} \mathrm{x} 1^{\wedge} 2^{*} \mathrm{y} 3-$ $684288^{*} \mathrm{u}[3]^{\wedge} 5^{*} \mathrm{y} 1^{*} \mathrm{x} 22^{\wedge} 2-159168^{*} \mathrm{u}[3]^{\wedge} 5^{*} \mathrm{y} 0{ }^{*} \mathrm{x} 2^{\wedge} 2-1152^{*} \mathrm{u}[3]^{\wedge} 5^{*} \mathrm{y} 1^{\wedge} 2^{*} \mathrm{x} 3+$ $9216^{*} \mathrm{u}[3]^{\wedge} 5^{*} \mathrm{y} 0 \wedge 2^{*} \mathrm{x} 2-103680^{*} \mathrm{u}[3]^{\wedge} 5^{*} \mathrm{y} 0{ }^{\wedge} 2^{*} \mathrm{x} 3-69120^{*} \mathrm{u}[3]^{\wedge} 5^{*} \mathrm{y} 1^{\wedge} 2^{*} \mathrm{x} 2-$ $150336^{*} \mathrm{u}[3]^{\wedge} 5^{*} \mathrm{y} 0^{*} \mathrm{x} 3^{\wedge} 2+17232^{*} \mathrm{u}[3]^{\wedge} 5^{*} \mathrm{y} 1^{*} \mathrm{x} 3^{\wedge} 2+25704^{*} \mathrm{u}[3]^{\wedge} 8^{*} \mathrm{y} 0^{*} \mathrm{x} 3^{*} \mathrm{x} 2+$ $14688^{*} \mathrm{u}[3]^{\wedge} 8^{*} \mathrm{y} 1^{*} \mathrm{x} 2^{*} \mathrm{x} 3-54432^{*} \mathrm{u}[3]^{*} \mathrm{x} 2^{\wedge} 2^{*} \mathrm{y} 2-67248^{*} \mathrm{u}[3]^{*} \mathrm{x} 3^{\wedge} 2^{*} \mathrm{y} 2+23616^{*} \mathrm{u}[3]^{*} \mathrm{x} 22^{\wedge} 2^{*} \mathrm{y} 3$ $-23616^{*} \mathrm{u}[3]^{*} \mathrm{x} 3^{\wedge} 2^{*} \mathrm{y} 3+665248^{*} \mathrm{u}[3]^{\wedge} 5^{*} \mathrm{x} 22^{\wedge} 2^{*} \mathrm{x} 3-305336^{*} \mathrm{u}[3]^{\wedge} 5^{*} \mathrm{x} 33^{\wedge} 2^{*} \mathrm{x} 2+$ $54432^{*} \mathrm{u}[3]^{\wedge} 5^{*} \mathrm{x} 22^{\wedge} 2^{*} \mathrm{y} 2-52560^{*} \mathrm{u}[3]^{\wedge} 5^{*} \mathrm{x} 3^{\wedge} 2^{*} \mathrm{y} 2-233280^{*} \mathrm{u}[3]^{\wedge} 5^{*} \mathrm{x} 2^{\wedge} 2^{*} \mathrm{y} 3+$ $23616^{*} \mathrm{u}[3]^{\wedge} 5^{*} \mathrm{x} 3^{\wedge} 2^{*} \mathrm{y} 3+1123168^{*} \mathrm{u}[3]^{\wedge} 3^{*} \mathrm{x} 22^{\wedge} 2^{*} \mathrm{x} 3-159176^{*} \mathrm{u}[3]^{\wedge} 3^{*} \mathrm{x} 3^{\wedge} 2^{*} \mathrm{x} 2+$ $118944^{*} \mathrm{u}[3]^{\wedge} 3^{*} \mathrm{x} 2^{\wedge} 2^{*} \mathrm{y} 2+201168^{*} \mathrm{u}[3]^{\wedge} 3^{*} \mathrm{x} 3^{\wedge} 2^{*} \mathrm{y} 2+149184^{*} \mathrm{u}[3]^{\wedge} 3^{*} \mathrm{x} 2^{\wedge} 2^{*} \mathrm{y} 3+$ $15552^{*} \mathrm{u}[3]^{\wedge} 3^{*} \mathrm{x} 3{ }^{\wedge} 2^{*} \mathrm{y} 3+5120^{*} \mathrm{x} 1{ }^{\wedge} 3-8960^{*} \mathrm{x} 00^{\wedge} 3+49392^{*} \mathrm{u}[3]^{\wedge} 8^{*} \mathrm{y} 00^{*} \mathrm{y} 2^{*} \mathrm{x} 3-$ $28224^{*} \mathrm{u}[3]^{\wedge} 8^{*} \mathrm{y} 0{ }^{*} \mathrm{y} 3^{*} \mathrm{x} 3-16128^{*} \mathrm{u}[3]^{\wedge} 8^{*} \mathrm{y} 1^{*} \mathrm{y} 3^{*} \mathrm{x} 3+28224^{*} \mathrm{u}[3]^{\wedge} 8^{*} \mathrm{y} 1^{*} \mathrm{y} 2^{*} \mathrm{x} 3-$ $3360^{*} \mathrm{y} 1^{*} 1^{*} \mathrm{x} 3-43008^{*} \mathrm{y} 0^{*} \mathrm{x} 0^{*} \mathrm{x} 2-3360^{*} \mathrm{y} 0^{*} \mathrm{x} 1^{*} \mathrm{x} 2+149352^{*} \mathrm{x} 3^{*} \mathrm{x} 0^{*} \mathrm{x} 1-1920^{*} \mathrm{y} 1^{*} \mathrm{x} 1^{*} \mathrm{x} 2-$ $10986^{*} \mathrm{x} 1{ }^{*} \mathrm{x} 2{ }^{*} \mathrm{x} 0+99840^{*} \mathrm{y} 2{ }^{*} \mathrm{x} 0 * \mathrm{x} 1-24576^{*} \mathrm{y} 1{ }^{*} \mathrm{x} 0{ }^{*} \mathrm{x} 2+7800^{*} \mathrm{y} 3^{*} \mathrm{x} 1{ }^{*} \mathrm{x} 0-$ $43008^{*} \mathrm{y} 1^{*} \mathrm{x} 0 * \mathrm{x} 3-79764^{*} \mathrm{u}[3]^{\wedge} 5^{*} \mathrm{x} 0 \wedge 3-42672^{*} \mathrm{u}[3]^{\wedge} 5^{*} \mathrm{x} 1^{\wedge} 3-71904^{*} \mathrm{u}[3]^{\wedge} 8^{*} \mathrm{x} 2^{*} \mathrm{y} 2^{*} \mathrm{x} 3+$ $41088^{*} \mathrm{u}[3]^{\wedge} 8^{*} \mathrm{x} 2^{*} \mathrm{y} 3^{*} \mathrm{x} 3+118368^{*} \mathrm{u}[3]^{*} \mathrm{x} 2^{*} \mathrm{y} 2^{*} \mathrm{x} 3+45216^{*} \mathrm{u}[3]^{*} \mathrm{x} 2^{*} \mathrm{y} 3^{*} \mathrm{x} 3+$ $91296^{*} \mathrm{u}[3]^{\wedge} 5^{*} \mathrm{x} 2{ }^{*} \mathrm{y} 2^{*} \mathrm{x} 3+74592^{*} \mathrm{u}[3]^{\wedge} 5^{*} \mathrm{x} 2{ }^{*} \mathrm{y} 3^{*} \mathrm{x} 3-207072^{*} \mathrm{u}[3]^{\wedge} 3^{*} \mathrm{x} 2{ }^{*} \mathrm{y} 2^{*} \mathrm{x} 3-$ $234144^{*} \mathrm{u}[3]^{\wedge} 3^{*} \mathrm{x} 2^{*} \mathrm{y} 3^{*} \mathrm{x} 3-2592^{*} \mathrm{u}[3]^{\wedge} 7^{*} \mathrm{x} 2{ }^{*} \mathrm{y} 2^{*} \mathrm{x} 3+114336^{*} \mathrm{u}[3]^{\wedge} 7^{*} \mathrm{x} 2^{*} \mathrm{y} 3^{*} \mathrm{x} 3+$ 
$122400^{*} \mathrm{u}[3]^{*} \mathrm{y} 2{ }^{*} \mathrm{x} 0{ }^{*} \mathrm{x} 1-43200^{*} \mathrm{u}[3]^{*} \mathrm{y} 3^{*} \mathrm{x} 1^{*} \mathrm{x} 0+75756^{*} \mathrm{u}[3]^{\wedge} 3^{*} \mathrm{x} 0^{\wedge} 3-49392^{*} \mathrm{y} 0{ }^{*} \mathrm{y} 2^{*} \mathrm{x} 3+$ $28224^{*} \mathrm{y} 0{ }^{*} \mathrm{y} 3^{*} \mathrm{x} 3+16128^{*} \mathrm{y} 1{ }^{*} \mathrm{y} 3{ }^{*} \mathrm{x} 3-28224^{*} \mathrm{y} 1{ }^{*} \mathrm{y} 2{ }^{*} \mathrm{x} 3-28224^{*} \mathrm{y} 0 * \mathrm{y} 2{ }^{*} \mathrm{x} 2+$ $16128^{*} \mathrm{y} 0{ }^{*} \mathrm{y} 3^{*} \mathrm{x} 2-16128^{*} \mathrm{y} 1^{*} \mathrm{y} 2{ }^{*} \mathrm{x} 2-21312^{*} \mathrm{u}[3]^{\wedge} 2^{*} \mathrm{x} 0{ }^{*} \mathrm{x} 1{ }^{*} \mathrm{y} 0+121536^{*} \mathrm{u}[3]^{\wedge} 2^{*} \mathrm{x} 0{ }^{*} \mathrm{x} 1{ }^{*} \mathrm{y} 1-$ $516096^{*} \mathrm{u}[3]^{\wedge} 4^{*} \mathrm{x} 00^{*} \mathrm{x} 1^{*} \mathrm{y} 0+4608^{*} \mathrm{u}[3]^{\wedge} 4^{*} \mathrm{x} 00^{*} \mathrm{x} 1^{*} \mathrm{y} 1-15552^{*} \mathrm{u}[3]^{\wedge} 6^{*} \mathrm{x} 0{ }^{*} \mathrm{x} 1^{*} \mathrm{y} 0-$ $57024^{*} \mathrm{u}[3]^{\wedge} 6^{*} \mathrm{x} 00^{*} \mathrm{x} 1^{*} \mathrm{y} 1+43008^{*} \mathrm{u}[3]^{\wedge} 8^{*} \mathrm{y} 00^{*} \mathrm{x} 0{ }^{*} \mathrm{x} 2+3360^{*} \mathrm{u}[3]^{\wedge} 8^{*} \mathrm{y} 00^{*} \mathrm{x} 1^{*} \mathrm{x} 2+$ $75264^{*} \mathrm{u}[3]^{\wedge} 8^{*} \mathrm{y} 00^{*} \mathrm{x} 0 * \mathrm{x} 3+5880^{*} \mathrm{u}[3]^{\wedge} 8^{*} \mathrm{y} 00^{*} \mathrm{x} 1{ }^{*} \mathrm{x} 3+24576^{*} \mathrm{u}[3]^{\wedge} 8^{*} \mathrm{y} 1{ }^{*} \mathrm{x} 0{ }^{*} \mathrm{x} 2+$ $1920^{*} \mathrm{u}[3]^{\wedge} 8^{*} \mathrm{y} 1^{*} \mathrm{x} 1{ }^{*} \mathrm{x} 2+43008^{*} \mathrm{u}[3]^{\wedge} 8^{*} \mathrm{y} 1^{*} \mathrm{x} 0{ }^{*} \mathrm{x} 3+3360^{*} \mathrm{u}[3]^{\wedge} 8^{*} \mathrm{y} 1^{*} \mathrm{x} 1^{*} \mathrm{x} 3-$ $159168^{*} \mathrm{u}[3]^{\wedge} 3^{*} \mathrm{y} 0^{*} \mathrm{x} 2 \wedge 2+7822^{*} \mathrm{x} 3^{*} \mathrm{x} 2{ }^{*} \mathrm{x} 1-2016^{*} \mathrm{u}[3]^{\wedge} 8^{*} \mathrm{y} 0 * \mathrm{x} 22^{\wedge} 2+51156^{*} \mathrm{u}[3]^{\wedge} 8^{*} \mathrm{y} 0{ }^{*} \mathrm{x} 3^{\wedge} 2$ $-1152^{*} \mathrm{u}[3]^{\wedge} 8^{*} \mathrm{y} 1^{*} \mathrm{x} 2^{\wedge} 2+29232^{*} \mathrm{u}[3]^{\wedge} 8^{*} \mathrm{y} 1^{*} \mathrm{x} 3^{\wedge} 2-104832^{*} \mathrm{u}[3]^{\wedge} 7^{*} \mathrm{y} 2^{\wedge} 2^{*} \mathrm{x} 3-$ $59904^{*} \mathrm{u}[3]^{\wedge} 7^{*} \mathrm{y} 3^{\wedge} 2^{*} \mathrm{x} 2+513504^{*} \mathrm{u}[3]^{\wedge} 3^{*} \mathrm{y} 2{ }^{*} \mathrm{x} 0{ }^{*} \mathrm{x} 1-8352^{*} \mathrm{u}[3]^{\wedge} 3^{*} \mathrm{y} 3^{*} \mathrm{x} 1^{*} \mathrm{x} 0+$ $726528^{*} \mathrm{u}[3]^{\wedge} 4^{*} \mathrm{x} 1^{*} \mathrm{x} 22^{*} \mathrm{x} 0-500736^{*} \mathrm{u}[3]^{\wedge} 4^{*} \mathrm{x} 3^{*} \mathrm{x} 00^{*} \mathrm{x} 1-180288^{*} \mathrm{u}[3]^{*} \mathrm{y} 0^{*} \mathrm{x} 22^{\wedge} 2$ $8064^{*} \mathrm{u}[3]^{*} \mathrm{y} 1^{\wedge} 2^{*} \mathrm{x} 3+64512^{*} \mathrm{u}[3]^{*} \mathrm{y} 0^{\wedge} 2^{*} \mathrm{x} 2+112896^{*} \mathrm{u}[3]^{*} \mathrm{y} 0{ }^{\wedge} 2^{*} \mathrm{x} 3-4608^{*} \mathrm{u}[3]^{*} \mathrm{y} 1^{\wedge} 2^{*} \mathrm{x} 2+$ $60480^{*} \mathrm{u}[3]^{*} \mathrm{y} 0 * \mathrm{x} 33^{\wedge} 2+25200^{*} \mathrm{u}[3]^{*} \mathrm{y} 1^{*} \mathrm{x} 3^{\wedge} 2-48384^{*} \mathrm{u}[3]^{\wedge} 4^{*} \mathrm{x} 2{ }^{*} \mathrm{y} 2{ }^{*} \mathrm{x} 0-$ $83808^{*} \mathrm{u}[3]^{\wedge} 3^{*} \mathrm{y} 2^{*} \mathrm{x} 1^{\wedge} 2-63360^{*} \mathrm{u}[3]^{\wedge} 3^{*} \mathrm{x} 1^{\wedge} 2^{*} \mathrm{y} 3+230400^{*} \mathrm{u}[3]^{\wedge} 4^{*} \mathrm{x} 1^{\wedge} 2^{*} \mathrm{x} 2+$ $306240^{*} \mathrm{u}[3]^{\wedge} 4^{*} \mathrm{x} 0^{\wedge} 2^{*} \mathrm{x} 2-73728^{*} \mathrm{u}[3]^{\wedge} 4^{*} \mathrm{x} 0^{\wedge} 2^{*} \mathrm{x} 3-66048^{*} \mathrm{u}[3]^{\wedge} 4^{*} \mathrm{x} 1^{\wedge} 2^{*} \mathrm{x} 3+$ $121368^{*} \mathrm{u}[3]^{\wedge} 2^{*} \mathrm{x} 3^{\wedge} 2^{*} \mathrm{x} 2+476736^{*} \mathrm{u}[3]^{\wedge} 2^{*} \mathrm{x} 22^{\wedge} 2^{*} \mathrm{x} 3+7472^{*} \mathrm{x} 1^{*} \mathrm{x} 22^{\wedge} 2+$ $46848^{*} \mathrm{u}[3]^{\wedge} 7^{*} \mathrm{y} 2^{*} \mathrm{x} 22^{*} \mathrm{x} 1+42048^{*} \mathrm{u}[3]^{\wedge} 7^{*} \mathrm{y} 2^{*} \mathrm{x} 1^{*} \mathrm{x} 3+12096^{*} \mathrm{u}[3]^{\wedge} 7^{*} \mathrm{x} 2{ }^{*} \mathrm{y} 2{ }^{*} \mathrm{x} 0+$ $343152^{*} \mathrm{u}[3]^{\wedge} 7^{*} \mathrm{x} 0{ }^{*} \mathrm{y} 3^{*} \mathrm{x} 2-186624^{*} \mathrm{u}[3]^{\wedge} 7^{*} \mathrm{x} 3^{*} \mathrm{y} 2^{*} \mathrm{x} 0+104832^{*} \mathrm{u}[3]^{*} \mathrm{y} 3^{*} \mathrm{y} 2^{*} \mathrm{x} 2+$ $59904^{*} \mathrm{u}[3]^{*} \mathrm{y} 3{ }^{*} \mathrm{y} 2{ }^{*} \mathrm{x} 3-47040^{*} \mathrm{u}[3]^{*} \mathrm{y} 3^{*} \mathrm{x} 0{ }^{*} \mathrm{x} 3+94464^{*} \mathrm{u}[3]^{*} \mathrm{y} 3^{*} \mathrm{x} 1{ }^{*} \mathrm{x} 2-$ $66816^{*} \mathrm{u}[3]^{*} \mathrm{x} 3^{*} \mathrm{y} 3^{*} \mathrm{x} 1+46848^{*} \mathrm{u}[3]^{*} \mathrm{y} 2^{*} \mathrm{x} 2{ }^{*} \mathrm{x} 1+42048^{*} \mathrm{u}[3]^{*} \mathrm{y} 2^{*} \mathrm{x} 1^{*} \mathrm{x} 3+$ $12096^{*} \mathrm{u}[3]^{*} \mathrm{x} 2^{*} \mathrm{y} 2^{*} \mathrm{x} 0+343152^{*} \mathrm{u}[3]^{*} \mathrm{x} 0{ }^{*} \mathrm{y} 3^{*} \mathrm{x} 2-186624^{*} \mathrm{u}[3]^{*} \mathrm{x} 3^{*} \mathrm{y} 2^{*} \mathrm{x} 0-$ $104832^{*} \mathrm{u}[3]^{\wedge} 3^{*} \mathrm{y} 3^{*} \mathrm{y} 2{ }^{*} \mathrm{x} 2-59904^{*} \mathrm{u}[3]^{\wedge} 3^{*} \mathrm{y} 3^{*} \mathrm{y} 2^{*} \mathrm{x} 3-64512^{*} \mathrm{u}[3]^{\wedge} 7^{*} \mathrm{y} 0{ }^{*} \mathrm{y} 2^{*} \mathrm{x} 0+$ $67968^{*} \mathrm{u}[3]^{\wedge} 7^{*} \mathrm{y} 0^{*} \mathrm{y} 2^{*} \mathrm{x} 1-112896^{*} \mathrm{u}[3]^{\wedge} 7^{*} \mathrm{y} 00^{*} \mathrm{y} 3^{*} \mathrm{x} 0-124416^{*} \mathrm{u}[3]^{\wedge} 7^{*} \mathrm{y} 00^{*} \mathrm{y} 3^{*} \mathrm{x} 1-$ $96768^{*} \mathrm{u}[3]^{\wedge} 7^{*} \mathrm{y} 1^{*} \mathrm{y} 2{ }^{*} \mathrm{x} 0+4608^{*} \mathrm{u}[3]^{\wedge} 7^{*} \mathrm{y} 1^{*} \mathrm{y} 2^{*} \mathrm{x} 1+74016^{*} \mathrm{u}[3]^{\wedge} 7^{*} \mathrm{y} 1^{*} \mathrm{y} 3{ }^{*} \mathrm{x} 0+$ $8064^{*} \mathrm{u}[3]^{\wedge} 7^{*} \mathrm{y} 1^{*} \mathrm{y} 3^{*} \mathrm{x} 1-34560^{*} \mathrm{u}[3]^{*} \mathrm{y} 1^{*} \mathrm{x} 2^{\wedge} 2-66816^{*} \mathrm{u}[3]^{\wedge} 7^{*} \mathrm{x} 3^{*} \mathrm{y} 3^{*} \mathrm{x} 1-$ $94464^{*} \mathrm{u}[3]^{\wedge} 2^{*} \mathrm{x} 00^{\wedge} 2^{*} \mathrm{y} 0-220320^{*} \mathrm{u}[3]^{\wedge} 2^{*} \mathrm{x} 0^{\wedge} 2^{*} \mathrm{y} 1+34560^{*} \mathrm{u}[3]^{\wedge} 2^{*} \mathrm{y} 0 * \mathrm{x} 1^{\wedge} 2$ $15552^{*} \mathrm{u}[3]^{\wedge} 2^{*} \mathrm{x} 1^{\wedge} 2^{*} \mathrm{y} 1+14112^{*} \mathrm{u}[3]^{\wedge} 4^{*} \mathrm{x} 0^{\wedge} 2^{*} \mathrm{x} 1-32256^{*} \mathrm{u}[3]^{\wedge} 4^{*} \mathrm{x} 0 \wedge 2^{*} \mathrm{y} 0+$ $243648^{*} \mathrm{u}[3]^{\wedge} 4^{*} \mathrm{x} 0^{\wedge} 2^{*} \mathrm{y} 1+195264^{*} \mathrm{u}[3]^{\wedge} 4^{*} \mathrm{x} 1^{\wedge} 2^{*} \mathrm{x} 0-117504^{*} \mathrm{u}[3]^{\wedge} 4^{*} \mathrm{y} 0^{*} \mathrm{x} 1^{\wedge} 2+$ $18432^{*} \mathrm{u}[3]^{\wedge} 4^{*} \mathrm{x} 1^{\wedge} 2^{*} \mathrm{y} 1+1152^{*} \mathrm{x} 3^{*} \mathrm{y} 3^{*} \mathrm{x} 1+28224^{*} \mathrm{u}[3]^{\wedge} 8^{*} \mathrm{y} 3^{\wedge} 2^{*} \mathrm{x} 0-16128^{*} \mathrm{u}[3]^{\wedge} 8^{*} \mathrm{y} 2^{\wedge} 2^{*} \mathrm{x} 1$ $-28224^{*} \mathrm{u}[3]^{\wedge} 8^{*} \mathrm{y} 2^{\wedge} 2^{*} \mathrm{x} 0+16128^{*} \mathrm{u}[3]^{\wedge} 8^{*} \mathrm{y} 3^{\wedge} 2^{*} \mathrm{x} 1-1152^{*} \mathrm{y} 2^{*} \mathrm{x} 2^{*} \mathrm{x} 1+78624^{*} \mathrm{x} 0^{\wedge} 2^{*} \mathrm{y} 3+$ $49956^{*} \mathrm{u}[3]^{*} \mathrm{x} 0^{\wedge} 3-43344^{*} \mathrm{u}[3]^{*} \mathrm{x} 1^{\wedge} 3+422064^{*} \mathrm{u}[3]^{\wedge} 5^{*} \mathrm{x} 2^{\wedge} 3-50964^{*} \mathrm{u}[3]^{\wedge} 5^{*} \mathrm{x} 3^{\wedge} 3+$ $70704^{*} \mathrm{u}[3]^{\wedge} 3^{*} \mathrm{x} 2^{\wedge} 3-114684^{*} \mathrm{u}[3]^{\wedge} 3^{*} \mathrm{x} 3^{\wedge} 3+132752^{*} \mathrm{u}[3]^{\wedge} 7^{*} \mathrm{x} 2^{\wedge} 3+28412^{*} \mathrm{u}[3]^{\wedge} 7^{*} \mathrm{x} 3^{\wedge} 3-$ $25704^{*} \mathrm{y} 0^{*} \mathrm{x} 3^{*} \mathrm{x} 2+65844^{*} \mathrm{y} 2^{*} \mathrm{x} 1^{*} \mathrm{x} 3+33264^{*} \mathrm{y} 3^{*} \mathrm{y} 2^{*} \mathrm{x} 0+215264^{*} \mathrm{u}[3]^{\wedge} 3^{*} \mathrm{x} 1^{\wedge} 2^{*} \mathrm{x} 3-$ $118266^{*} \mathrm{u}[3]^{\wedge} 7^{*} \mathrm{x} 00^{\wedge} 2^{*} \mathrm{x} 2+124688^{*} \mathrm{u}[3]^{\wedge} 7^{*} \mathrm{x} 1^{\wedge} 2^{*} \mathrm{x} 2+38780^{*} \mathrm{u}[3]^{\wedge} 7^{*} \mathrm{x} 00^{\wedge} 2^{*} \mathrm{x} 3-$ $177360^{*} \mathrm{u}[3]^{\wedge} 7^{*} \mathrm{x} 1^{\wedge} 2^{*} \mathrm{x} 3+13440^{*} \mathrm{u}[3]^{*} \mathrm{x} 0^{\wedge} 2^{*} \mathrm{y} 0-161424^{*} \mathrm{u}[3]^{*} \mathrm{x} 0^{\wedge} 2^{*} \mathrm{y} 1-$ $85824^{*} \mathrm{u}[3]^{*} \mathrm{y} 0{ }^{*} \mathrm{x} 11^{\wedge} 2+12288^{*} \mathrm{u}[3]^{*} \mathrm{x} 1^{\wedge} 2^{*} \mathrm{y} 1-34560^{*} \mathrm{u}[3]^{\wedge} 7^{*} \mathrm{y} 1^{*} \mathrm{x} 2^{\wedge} 2-$ $180288^{*} \mathrm{u}[3]^{\wedge} 7^{*} \mathrm{y} 0^{*} \mathrm{x} 2^{\wedge} 2+43028^{*} \mathrm{u}[3]^{*} \mathrm{x} 3^{\wedge} 3-82800^{*} \mathrm{u}[3]^{\wedge} 3^{*} \mathrm{x} 0^{\wedge} 2^{*} \mathrm{y} 2-$ $197784^{*} \mathrm{u}[3]^{\wedge} 3^{*} \mathrm{x} 0^{\wedge} 2^{*} \mathrm{y} 3+16560^{*} \mathrm{u}[3]^{\wedge} 3^{*} \mathrm{x} 1^{\wedge} 2^{*} \mathrm{x} 0-28848^{*} \mathrm{u}[3]^{\wedge} 3^{*} \mathrm{x} 1^{\wedge} 3+$ $8064^{*} \mathrm{u}[3]^{*} \mathrm{x} 00^{*} \mathrm{x} 1^{*} \mathrm{y} 1-46080^{*} \mathrm{u}[3]^{\wedge} 7^{*} \mathrm{y} 1^{*} \mathrm{x} 2{ }^{*} \mathrm{x} 3+30168^{*} \mathrm{u}[3]^{\wedge} 7^{*} \mathrm{y} 1^{*} \mathrm{x} 1^{*} \mathrm{x} 3+$ $114336^{*} \mathrm{u}[3]^{\wedge} 7^{*} \mathrm{y} 0^{*} \mathrm{x} 0^{*} \mathrm{x} 2+8640^{*} \mathrm{u}[3]^{\wedge} 7^{*} \mathrm{y} 0^{*} \mathrm{x} 1^{*} \mathrm{x} 2+9216^{*} \mathrm{u}[3]^{\wedge} 7^{*} \mathrm{y} 1^{*} \mathrm{x} 1^{*} \mathrm{x} 2-$ $5400^{*} \mathrm{u}[3]^{\wedge} 7^{*} \mathrm{y} 1^{*} \mathrm{x} 0^{*} \mathrm{x} 2-106560^{*} \mathrm{u}[3]^{\wedge} 7^{*} \mathrm{y} 1^{*} \mathrm{x} 0^{*} \mathrm{x} 3-280944^{*} \mathrm{u}[3]^{\wedge} 7^{*} \mathrm{y} 0^{*} \mathrm{x} 3^{*} \mathrm{x} 2+$ $28800^{*} \mathrm{u}[3]^{\wedge} 7^{*} \mathrm{y} 0{ }^{*} \mathrm{y} 1^{*} \mathrm{x} 2+50400^{*} \mathrm{u}[3]^{\wedge} 7^{*} \mathrm{y} 00^{*} \mathrm{y} 1^{*} \mathrm{x} 3+3528^{*} \mathrm{u}[3]^{\wedge} 7^{*} \mathrm{y} 0 * \mathrm{x} 0 * \mathrm{x} 3+$ $24480^{*} \mathrm{u}[3]^{\wedge} 7^{*} \mathrm{y} 0{ }^{*} \mathrm{x} 1^{*} \mathrm{x} 3+295680^{*} \mathrm{u}[3]^{\wedge} 3^{*} \mathrm{y} 1^{*} \mathrm{x} 2{ }^{*} \mathrm{x} 3-37368^{*} \mathrm{u}[3]^{\wedge} 3^{*} \mathrm{y} 1^{*} \mathrm{x} 1^{*} \mathrm{x} 3-$ $206496^{*} \mathrm{u}[3]^{\wedge} 3^{*} \mathrm{y} 0^{*} \mathrm{x} 0^{*} \mathrm{x} 2+212544^{*} \mathrm{u}[3]^{\wedge} 3^{*} \mathrm{y} 0^{*} \mathrm{x} 1^{*} \mathrm{x} 2+202752^{*} \mathrm{u}[3]^{\wedge} 3^{*} \mathrm{y} 1^{*} \mathrm{x} 1^{*} \mathrm{x} 2-$ $683208^{*} \mathrm{u}[3]^{\wedge} 3^{*} \mathrm{y} 1^{*} \mathrm{x} 0{ }^{*} \mathrm{x} 2+283968^{*} \mathrm{u}[3]^{\wedge} 3^{*} \mathrm{y} 1^{*} \mathrm{x} 0^{*} \mathrm{x} 3-51156^{*} \mathrm{x} 2^{*} \mathrm{y} 2^{*} \mathrm{x} 0-7616^{*} \mathrm{x} 0{ }^{*} \mathrm{x} 3^{\wedge} 2-$ 
$158976^{*} \mathrm{u}[3]^{\wedge} 6^{*} \mathrm{y} 1^{*} \mathrm{y} 3^{*} \mathrm{x} 2+24192^{*} \mathrm{u}[3]^{\wedge} 2^{*} \mathrm{y} 0^{*} \mathrm{y} 2^{*} \mathrm{x} 2-51156^{*} \mathrm{y} 0^{*} \mathrm{x} 3^{\wedge} 2-29232^{*} \mathrm{y} 1^{*} \mathrm{x} 3{ }^{\wedge} 2+$ $57089^{*} \mathrm{x} 2^{*} \mathrm{x} 0^{*} \mathrm{x} 3-68320^{*} \mathrm{u}[3]^{\wedge} 6^{*} \mathrm{x} 1^{*} \mathrm{x} 22^{\wedge} 2+70604^{*} \mathrm{u}[3]^{\wedge} 6^{*} \mathrm{x} 0^{*} \mathrm{x} 2^{\wedge} 2-55784^{*} \mathrm{u}[3]^{\wedge} 6^{*} \mathrm{x} 0^{*} \mathrm{x} 3^{\wedge} 2$ $+169642^{*} \mathrm{u}[3]^{\wedge} 6^{*} \mathrm{x} 3^{\wedge} 2^{*} \mathrm{x} 1+2496^{*} \mathrm{u}[3]^{\wedge} 6^{*} \mathrm{x} 22^{\wedge} 2^{*} \mathrm{x} 3-383448^{*} \mathrm{u}[3]^{\wedge} 6^{*} \mathrm{x} 3^{\wedge} 2^{*} \mathrm{x} 2$ $326592^{*} \mathrm{u}[3]^{\wedge} 6^{*} \mathrm{y} 0^{*} \mathrm{x} 2^{\wedge} 2+20520^{*} \mathrm{u}[3]^{\wedge} 6^{*} \mathrm{y} 0^{*} \mathrm{x} 3^{\wedge} 2-14400^{*} \mathrm{u}[3]^{\wedge} 6^{*} \mathrm{y} 1^{*} \mathrm{x} 2^{\wedge} 2-$ $576^{*} \mathrm{u}[3]^{\wedge} 6^{*} \mathrm{y} 1{ }^{*} \mathrm{x} 3{ }^{\wedge} 2+24552^{*} \mathrm{x} 00^{*} \mathrm{y} 3^{*} \mathrm{x} 2-63216^{*} \mathrm{u}[3]^{\wedge} 4^{*} \mathrm{x} 0^{\wedge} 3-70848^{*} \mathrm{u}[3]^{\wedge} 4^{*} \mathrm{x} 1^{\wedge} 3-$ $334368^{*} \mathrm{u}[3]^{\wedge} 6^{*} \mathrm{y} 0 * \mathrm{y} 2 * \mathrm{x} 3+54144^{*} \mathrm{u}[3]^{\wedge} 6^{*} \mathrm{y} 00^{*} \mathrm{y} 3{ }^{*} \mathrm{x} 3+13824^{*} \mathrm{u}[3]^{\wedge} 6^{*} \mathrm{y} 1^{*} \mathrm{y} 3{ }^{*} \mathrm{x} 3+$ $5760^{*} \mathrm{u}[3]^{\wedge} 6^{*} \mathrm{y} 1^{*} \mathrm{y} 2^{*} \mathrm{x} 3-24192^{*} \mathrm{u}[3]^{\wedge} 6^{*} \mathrm{y} 0^{*} \mathrm{y} 2^{*} \mathrm{x} 2+253440^{*} \mathrm{u}[3]^{\wedge} 6^{*} \mathrm{y} 00^{*} \mathrm{y} 3^{*} \mathrm{x} 2+$ $225792^{*} \mathrm{u}[3]^{\wedge} 6^{*} \mathrm{y} 1{ }^{*} \mathrm{y} 2^{*} \mathrm{x} 2+660480^{*} \mathrm{u}[3]^{\wedge} 4^{*} \mathrm{x} 22^{\wedge} 2^{*} \mathrm{x} 3-194496^{*} \mathrm{u}[3]^{\wedge} 4^{*} \mathrm{x} 3{ }^{\wedge} 2^{*} \mathrm{x} 2-$ $99072^{*} \mathrm{u}[3]^{\wedge} 4^{*} \mathrm{y} 0{ }^{*} \mathrm{x} 22^{\wedge} 2-140544^{*} \mathrm{u}[3]^{\wedge} 4^{*} \mathrm{y} 0{ }^{*} \mathrm{x} 3{ }^{\wedge} 2-433152^{*} \mathrm{u}[3]^{\wedge} 4^{*} \mathrm{y} 1{ }^{*} \mathrm{x} 2{ }^{\wedge} 2-$ $23616^{*} \mathrm{u}[3]^{\wedge} 4^{*} \mathrm{y} 1^{*} \mathrm{x} 3^{\wedge} 2+895304^{*} \mathrm{u}[3]^{\wedge} 3^{*} \mathrm{x} 1^{*} \mathrm{x} 2{ }^{*} \mathrm{x} 0-784762^{*} \mathrm{u}[3]^{\wedge} 3^{*} \mathrm{x} 3^{*} \mathrm{x} 0^{*} \mathrm{x} 1+$ $227784^{*} \mathrm{u}[3]^{\wedge} 7^{*} \mathrm{x} 1{ }^{*} \mathrm{x} 2{ }^{*} \mathrm{x} 0+11586^{*} \mathrm{u}[3]^{\wedge} 7^{*} \mathrm{x} 3^{*} \mathrm{x} 00^{*} \mathrm{x} 1+763184^{*} \mathrm{u}[3]^{\wedge} 5^{*} \mathrm{x} 1^{*} \mathrm{x} 2{ }^{*} \mathrm{x} 0-$ $308122^{*} \mathrm{u}[3]^{\wedge} 5^{*} \mathrm{x} 3^{*} \mathrm{x} 00^{*} \mathrm{x} 1+531180^{*} \mathrm{u}[3]^{\wedge} 2^{*} \mathrm{x} 1{ }^{*} \mathrm{x} 2{ }^{*} \mathrm{x} 0-78000^{*} \mathrm{u}[3]^{\wedge} 2^{*} \mathrm{x} 3{ }^{*} \mathrm{x} 0{ }^{*} \mathrm{x} 1+$ $829634^{*} \mathrm{u}[3]^{\wedge} 2^{*} \mathrm{x} 22^{*} \mathrm{x} 0{ }^{*} \mathrm{x} 3+214084^{*} \mathrm{u}[3]^{\wedge} 2^{*} \mathrm{x} 3^{*} \mathrm{x} 22^{*} \mathrm{x} 1-46080^{*} \mathrm{u}[3]^{\wedge} 7^{*} \mathrm{x} 2^{*} \mathrm{x} 0{ }^{*} \mathrm{x} 3+$ $95460^{*} \mathrm{u}[3]^{\wedge} 7^{*} \mathrm{x} 3^{*} \mathrm{x} 2^{*} \mathrm{x} 1-140544^{*} \mathrm{u}[3]^{\wedge} 5^{*} \mathrm{x} 2^{*} \mathrm{x} 0{ }^{*} \mathrm{x} 3-655572^{*} \mathrm{u}[3]^{\wedge} 5^{*} \mathrm{x} 3^{*} \mathrm{x} 2^{*} \mathrm{x} 1+$ $490752^{*} \mathrm{u}[3]^{\wedge} 3^{*} \mathrm{x} 2{ }^{*} \mathrm{x} 0{ }^{*} \mathrm{x} 3-64512^{*} \mathrm{u}[3]^{*} \mathrm{y} 0{ }^{*} \mathrm{y} 2{ }^{*} \mathrm{x} 0+67968^{*} \mathrm{u}[3]^{*} \mathrm{y} 0 * \mathrm{y} 2{ }^{*} \mathrm{x} 1-$ $112896^{*} \mathrm{u}[3]^{*} \mathrm{y} 0{ }^{*} \mathrm{y} 3^{*} \mathrm{x} 0-124416^{*} \mathrm{u}[3]^{*} \mathrm{y} 0{ }^{*} \mathrm{y} 3{ }^{*} \mathrm{x} 1-96768^{*} \mathrm{u}[3]^{*} \mathrm{y} 1^{*} \mathrm{y} 2^{*} \mathrm{x} 0+$ $4608^{*} \mathrm{u}[3]^{*} \mathrm{y} 1^{*} \mathrm{y} 2^{*} \mathrm{x} 1+41892^{*} \mathrm{u}[3]^{\wedge} 7^{*} \mathrm{x} 0^{\wedge} 3-38736^{*} \mathrm{u}[3]^{\wedge} 7^{*} \mathrm{x} 1^{\wedge} 3-9216^{*} \mathrm{u}[3]^{\wedge} 5^{*} \mathrm{y} 00^{*} \mathrm{y} 2^{*} \mathrm{x} 0-$ $657792^{*} \mathrm{u}[3]^{\wedge} 5^{*} \mathrm{y} 0 * \mathrm{y} 2^{*} \mathrm{x} 1+103680^{*} \mathrm{u}[3]^{\wedge} 5 * \mathrm{y} 0 * \mathrm{y} 3^{*} \mathrm{x} 0+50688^{*} \mathrm{u}[3]^{\wedge} 5^{*} \mathrm{y} 00^{*} \mathrm{y} 3^{*} \mathrm{x} 1+$ $105984^{*} \mathrm{u}[3]^{\wedge} 5^{*} \mathrm{y} 1^{*} \mathrm{y} 2^{*} \mathrm{x} 0+69120^{*} \mathrm{u}[3]^{\wedge} 5^{*} \mathrm{y} 1^{*} \mathrm{y} 2^{*} \mathrm{x} 1-72864^{*} \mathrm{u}[3]^{\wedge} 5^{*} \mathrm{y} 1^{*} \mathrm{y} 3^{*} \mathrm{x} 0+$ $1152^{*} \mathrm{u}[3]^{\wedge} 5^{*} \mathrm{y} 1{ }^{*} \mathrm{y} 3{ }^{*} \mathrm{x} 1+71904^{*} \mathrm{x} 2{ }^{*} \mathrm{y} 2 * \mathrm{x} 3-41088^{*} \mathrm{x} 2{ }^{*} \mathrm{y} 3{ }^{*} \mathrm{x} 3-75264{ }^{*} \mathrm{y} 0{ }^{*} \mathrm{x} 0{ }^{*} \mathrm{x} 3-$ $5880^{*} \mathrm{y} 0^{*} \mathrm{x} 1{ }^{*} \mathrm{x} 3-21504^{*} \mathrm{u}[3]^{\wedge} 6^{*} \mathrm{x} 22^{\wedge} 2^{*} \mathrm{y} 2-152592^{*} \mathrm{u}[3]^{\wedge} 6^{*} \mathrm{x} 3^{\wedge} 2^{*} \mathrm{y} 2-292224^{*} \mathrm{u}[3]^{\wedge} 6^{*} \mathrm{x} 2{ }^{\wedge} 2^{*} \mathrm{y} 3$ $+30144^{*} \mathrm{u}[3]^{\wedge} 6^{*} \mathrm{x} 3{ }^{\wedge} 2^{*} \mathrm{y} 3-172800^{*} \mathrm{u}[3]^{\wedge} 4^{*} \mathrm{x} 2{ }^{*} \mathrm{x} 00^{*} \mathrm{x} 3-399600^{*} \mathrm{u}[3]^{\wedge} 4^{*} \mathrm{x} 3{ }^{*} \mathrm{x} 2{ }^{*} \mathrm{x} 1-$ $57089^{*} \mathrm{u}[3]^{\wedge} 8^{*} \mathrm{x} 2{ }^{*} \mathrm{x} 0{ }^{*} \mathrm{x} 3-7822^{*} \mathrm{u}[3]^{\wedge} 8^{*} \mathrm{x} 3^{*} \mathrm{x} 2{ }^{*} \mathrm{x} 1+106944^{*} \mathrm{u}[3]^{\wedge} 6^{*} \mathrm{x} 2{ }^{*} \mathrm{y} 2{ }^{*} \mathrm{x} 3+$ $212736^{*} \mathrm{u}[3]^{\wedge} 6^{*} \mathrm{x} 2^{*} \mathrm{y} 3^{*} \mathrm{x} 3+433056^{*} \mathrm{u}[3]^{\wedge} 6^{*} \mathrm{x} 22^{\wedge} 3-21996^{*} \mathrm{u}[3]^{\wedge} 6^{*} \mathrm{x} 3^{\wedge} 3-$ $31680^{*} \mathrm{u}[3]^{\wedge} 2^{*} \mathrm{y} 3{ }^{*} \mathrm{x} 1{ }^{*} \mathrm{x} 2+538752^{*} \mathrm{u}[3]^{\wedge} 2^{*} \mathrm{y} 2{ }^{*} \mathrm{x} 0{ }^{*} \mathrm{x} 1-161040^{*} \mathrm{u}[3]^{\wedge} 2^{*} \mathrm{y} 3{ }^{*} \mathrm{x} 1{ }^{*} \mathrm{x} 0+$ $36288^{*} \mathrm{u}[3]^{\wedge} 2^{*} \mathrm{x} 3^{*} \mathrm{y} 3^{*} \mathrm{x} 1+16128^{*} \mathrm{u}[3]^{\wedge} 2^{*} \mathrm{y} 2^{*} \mathrm{x} 2^{*} \mathrm{x} 1+141480^{*} \mathrm{u}[3]^{\wedge} 2^{*} \mathrm{y} 2^{*} \mathrm{x} 1^{*} \mathrm{x} 3-$ $80928^{*} \mathrm{u}[3]^{\wedge} 2^{*} \mathrm{y} 3^{*} \mathrm{y} 2{ }^{*} \mathrm{x} 0+221976^{*} \mathrm{u}[3]^{\wedge} 2^{*} \mathrm{x} 2{ }^{*} \mathrm{y} 2{ }^{*} \mathrm{x} 0+244080^{*} \mathrm{u}[3]^{\wedge} 2^{*} \mathrm{x} 0{ }^{*} \mathrm{y} 3{ }^{*} \mathrm{x} 2-$ $289440^{*} \mathrm{u}[3]^{\wedge} 2^{*} \mathrm{x} 3^{*} \mathrm{y} 2^{*} \mathrm{x} 0-153216^{*} \mathrm{u}[3]^{\wedge} 2^{*} \mathrm{y} 3^{*} \mathrm{y} 2^{*} \mathrm{x} 1-51156^{*} \mathrm{u}[3]^{\wedge} 8^{*} \mathrm{y} 3^{*} \mathrm{x} 0^{*} \mathrm{x} 3+$ $2016^{*} \mathrm{u}[3]^{\wedge} 8^{*} \mathrm{y} 3^{*} \mathrm{x} 1^{*} \mathrm{x} 2-1152^{*} \mathrm{u}[3]^{\wedge} 8^{*} \mathrm{x} 3^{*} \mathrm{y} 3^{*} \mathrm{x} 1+1152^{*} \mathrm{u}[3]^{\wedge} 8^{*} \mathrm{y} 2^{*} \mathrm{x} 2^{*} \mathrm{x} 1-$ $65844^{*} \mathrm{u}[3]^{\wedge} 8^{*} \mathrm{y} 2{ }^{*} \mathrm{x} 1{ }^{*} \mathrm{x} 3-33264^{*} \mathrm{u}[3]^{\wedge} 8^{*} \mathrm{y} 3^{*} \mathrm{y} 2^{*} \mathrm{x} 0+51156^{*} \mathrm{u}[3]^{\wedge} 8^{*} \mathrm{x} 22^{*} \mathrm{y} 2{ }^{*} \mathrm{x} 0-$ $24552^{*} \mathrm{u}[3]^{\wedge} 8^{*} \mathrm{x} 0^{*} \mathrm{y} 3^{*} \mathrm{x} 2-29232^{*} \mathrm{u}[3]^{\wedge} 8^{*} \mathrm{x} 3^{*} \mathrm{y} 2^{*} \mathrm{x} 0-19008^{*} \mathrm{u}[3]^{\wedge} 8^{*} \mathrm{y} 3^{*} \mathrm{y} 2^{*} \mathrm{x} 1-$ $480336^{*} \mathrm{u}[3]^{\wedge} 3^{*} \mathrm{y} 0 * \mathrm{x} 3^{*} \mathrm{x} 2+551808^{*} \mathrm{u}[3]^{\wedge} 3^{*} \mathrm{y} 0{ }^{*} \mathrm{y} 1^{*} \mathrm{x} 2+22176^{*} \mathrm{u}[3]^{\wedge} 3^{*} \mathrm{y} 0{ }^{*} \mathrm{y} 1^{*} \mathrm{x} 3+$ $213336^{*} \mathrm{u}[3]^{\wedge} 3^{*} \mathrm{y} 00^{*} \mathrm{x} 0 * \mathrm{x} 3-19296^{*} \mathrm{u}[3]^{\wedge} 3^{*} \mathrm{y} 0{ }^{*} \mathrm{x} 1^{*} \mathrm{x} 3-775680^{*} \mathrm{u}[3]^{\wedge} 5^{*} \mathrm{x} 00^{*} \mathrm{x} 1^{*} \mathrm{y} 0+$ $89472^{*} \mathrm{u}[3]^{\wedge} 5^{*} \mathrm{x} 0^{*} \mathrm{x} 1^{*} \mathrm{y} 1-4608^{*} \mathrm{u}[3]^{\wedge} 7^{*} \mathrm{x} 00^{*} \mathrm{x} 1^{*} \mathrm{y} 0+8064^{*} \mathrm{u}[3]^{\wedge} 7^{*} \mathrm{x} 0{ }^{*} \mathrm{x} 1^{*} \mathrm{y} 1+$ $43200^{*} \mathrm{u}[3]^{\wedge} 3^{*} \mathrm{y} 3^{*} \mathrm{x} 00^{*} \mathrm{x} 3-768^{*} \mathrm{u}[3]^{\wedge} 3^{*} \mathrm{y} 3^{*} \mathrm{x} 1^{*} \mathrm{x} 2+36096^{*} \mathrm{u}[3]^{\wedge} 3^{*} \mathrm{x} 3^{*} \mathrm{y} 3^{*} \mathrm{x} 1+$ $702720^{*} \mathrm{u}[3]^{\wedge} 3^{*} \mathrm{y} 2^{*} \mathrm{x} 2^{*} \mathrm{x} 1-287808^{*} \mathrm{u}[3]^{\wedge} 3^{*} \mathrm{y} 2^{*} \mathrm{x} 1^{*} \mathrm{x} 3+81600^{*} \mathrm{u}[3]^{\wedge} 3^{*} \mathrm{x} 2^{*} \mathrm{y} 2^{*} \mathrm{x} 0-$ $331440^{*} \mathrm{u}[3]^{\wedge} 3^{*} \mathrm{x} 00^{*} \mathrm{y} 3^{*} \mathrm{x} 2+155904^{*} \mathrm{u}[3]^{\wedge} 3^{*} \mathrm{x} 3^{*} \mathrm{y} 2^{*} \mathrm{x} 0+62208^{*} \mathrm{u}[3]^{\wedge} 6^{*} \mathrm{x} 0 \wedge 2^{*} \mathrm{y} 0-$ $15264^{*} \mathrm{u}[3]^{\wedge} 6^{*} \mathrm{x} 0^{\wedge} 2^{*} \mathrm{y} 1-211968^{*} \mathrm{u}[3]^{\wedge} 6^{*} \mathrm{y} 0^{*} \mathrm{x} 1^{\wedge} 2+33984^{*} \mathrm{u}[3]^{\wedge} 6^{*} \mathrm{x} 1^{\wedge} 2^{*} \mathrm{y} 1-$ $4608^{*} \mathrm{u}[3]^{*} \mathrm{x} 00^{*} \mathrm{x} 1^{*} \mathrm{y} 0+29952^{*} \mathrm{u}[3]^{\wedge} 6^{*} \mathrm{y} 1^{\wedge} 2^{*} \mathrm{x} 0+239616^{*} \mathrm{u}[3]^{\wedge} 6^{*} \mathrm{y} 0^{\wedge} 2^{*} \mathrm{x} 1-$ $73632^{*} \mathrm{u}[3]^{\wedge} 2^{*} \mathrm{x} 2{ }^{\wedge} 3+21996^{*} \mathrm{u}[3]^{\wedge} 2^{*} \mathrm{x} 3^{\wedge} 3+44928^{*} \mathrm{y} 2^{*} \mathrm{x} 1^{\wedge} 2-46080^{*} \mathrm{u}[3]^{*} \mathrm{y} 1^{*} \mathrm{x} 2^{*} \mathrm{x} 3+$ $38232{ }^{*} \mathrm{u}[3]^{*} \mathrm{y} 1{ }^{*} \mathrm{x} 1{ }^{*} \mathrm{x} 3+49824{ }^{*} \mathrm{u}[3]^{*} \mathrm{y} 0 *^{*} \mathrm{x} 0{ }^{*} \mathrm{x} 2+16704{ }^{*} \mathrm{u}[3]{ }^{*} \mathrm{y} 0{ }^{*} \mathrm{x} 1{ }^{*} \mathrm{x} 2+$ $13824^{*} \mathrm{u}[3]^{*} \mathrm{y} 1{ }^{*} \mathrm{x} 1{ }^{*} \mathrm{x} 2-42264 * \mathrm{u}[3]^{*} \mathrm{y} 1{ }^{*} \mathrm{x} 0{ }^{*} \mathrm{x} 2-171072 * \mathrm{u}[3]^{*} \mathrm{y} 1{ }^{*} \mathrm{x} 0{ }^{*} \mathrm{x} 3-$ $280944^{*} \mathrm{u}[3]^{*} \mathrm{y} 0{ }^{*} \mathrm{x} 3^{*} \mathrm{x} 2+28800^{*} \mathrm{u}[3]^{*} \mathrm{y} 0{ }^{*} \mathrm{y} 1{ }^{*} \mathrm{x} 2+50400^{*} \mathrm{u}[3]^{*} \mathrm{y} 0{ }^{*} \mathrm{y} 1{ }^{*} \mathrm{x} 3-$ $109368^{*} \mathrm{u}[3]^{*} \mathrm{y} 00^{*} \mathrm{x} 0{ }^{*} \mathrm{x} 3-283096^{*} \mathrm{u}[3]^{\wedge} 6^{*} \mathrm{x} 0^{\wedge} 2^{*} \mathrm{x} 1-15888^{*} \mathrm{u}[3]^{\wedge} 6^{*} \mathrm{x} 0^{\wedge} 2^{*} \mathrm{y} 2+$ $174528^{*} \mathrm{u}[3]^{\wedge} 6^{*} \mathrm{x} 0^{\wedge} 2^{*} \mathrm{y} 3-93248^{*} \mathrm{u}[3]^{\wedge} 6^{*} \mathrm{x} 1^{\wedge} 2^{*} \mathrm{x} 0-48384^{*} \mathrm{u}[3]^{\wedge} 6^{*} \mathrm{y} 2^{*} \mathrm{x} 1^{\wedge} 2+$ 
$15168^{*} \mathrm{u}[3]^{\wedge} 6{ }^{*} \mathrm{x} 1{ }^{\wedge} 2^{*} \mathrm{y} 3+6090^{*} \mathrm{u}[3]^{\wedge} 8^{*} \mathrm{x} 0 \wedge 2^{*} \mathrm{x} 3-108868^{*} \mathrm{u}[3]^{\wedge} 8^{*} \mathrm{x} 00^{\wedge} 2^{*} \mathrm{x} 1+$ $74460 * \mathrm{u}[3]^{\wedge} 8^{*} \mathrm{x} 0 \wedge 2^{*} \mathrm{x} 2-37128^{*} \mathrm{u}[3]^{\wedge} 8^{*} \mathrm{x} 0 \wedge 2^{*} \mathrm{y} 2-78624^{*} \mathrm{u}[3]^{\wedge} 8^{*} \mathrm{x} 0^{\wedge} 2^{*} \mathrm{y} 3-$ $74096^{*} \mathrm{u}[3]^{\wedge} 8^{*} \mathrm{x} 1^{\wedge} 2^{*} \mathrm{x} 0+3072^{*} \mathrm{u}[3]^{\wedge} 8^{*} \mathrm{x} 1^{\wedge} 2^{*} \mathrm{x} 2-50394^{*} \mathrm{u}[3]^{\wedge} 8^{*} \mathrm{x} 1^{\wedge} 2^{*} \mathrm{x} 3-$ $44928^{*} \mathrm{u}[3]^{\wedge} 8^{*} \mathrm{y} 2^{*} \mathrm{x} 1^{\wedge} 2+21216^{*} \mathrm{u}[3]^{\wedge} 8^{*} \mathrm{x} 1{ }^{\wedge} 2^{*} \mathrm{y} 3-104832^{*} \mathrm{u}[3]^{*} \mathrm{y} 2^{\wedge} 2^{*} \mathrm{x} 3-$ $59904^{*} \mathrm{u}[3]^{*} \mathrm{y} 3^{\wedge} 2^{*} \mathrm{x} 2+46528^{*} \mathrm{u}[3]^{\wedge} 2^{*} \mathrm{x} 1^{\wedge} 3-107136^{*} \mathrm{u}[3]^{\wedge} 3^{*} \mathrm{x} 0^{\wedge} 2^{*} \mathrm{y} 0+$ $173136^{*} \mathrm{u}[3]^{\wedge} 3^{*} \mathrm{x} 0^{\wedge} 2^{*} \mathrm{y} 1-159936^{*} \mathrm{u}[3]^{\wedge} 3^{*} \mathrm{y} 0^{*} \mathrm{x} 1^{\wedge} 2+18432^{*} \mathrm{u}[3]^{\wedge} 3^{*} \mathrm{x} 1^{\wedge} 2^{*} \mathrm{y} 1+$ $108192^{*} \mathrm{u}[3]^{*} \mathrm{x} 22^{\wedge} 2^{*} \mathrm{x} 3-29952^{*} \mathrm{u}[3]^{\wedge} 2^{*} \mathrm{y} 1^{\wedge} 2^{*} \mathrm{x} 0-239616^{*} \mathrm{u}[3]^{\wedge} 2^{*} \mathrm{y} 0^{\wedge} 2^{*} \mathrm{x} 1-$ $322068^{*} \mathrm{u}[3]^{\wedge} 7^{*} \mathrm{x} 0^{*} \mathrm{x} 2^{\wedge} 2-38736^{*} \mathrm{u}[3]^{\wedge} 7^{*} \mathrm{x} 1^{*} \mathrm{x} 2^{\wedge} 2+41892^{*} \mathrm{u}[3]^{\wedge} 7^{*} \mathrm{x} 0^{*} \mathrm{x} 3^{\wedge} 2+$ $32184^{*} \mathrm{u}[3]^{\wedge} 7^{*} \mathrm{x} 3^{\wedge} 2^{*} \mathrm{x} 1+644484^{*} \mathrm{u}[3]^{\wedge} 5^{*} \mathrm{x} 0^{*} \mathrm{x} 2^{\wedge} 2-42672^{*} \mathrm{u}[3]^{\wedge} 5^{*} \mathrm{x} 1^{*} \mathrm{x} 2^{\wedge} 2-$ $79764^{*} \mathrm{u}[3]^{\wedge} 5^{*} \mathrm{x} 00^{*} \mathrm{x} 3^{\wedge} 2+86664^{*} \mathrm{u}[3]^{\wedge} 5^{*} \mathrm{x} 3^{\wedge} 2^{*} \mathrm{x} 1+414084^{*} \mathrm{u}[3]^{\wedge} 3^{*} \mathrm{x} 0^{*} \mathrm{x} 22^{\wedge} 2-$ $28848^{*} \mathrm{u}[3]^{\wedge} 3^{*} \mathrm{x} 1^{*} \mathrm{x} 2^{\wedge} 2+75756^{*} \mathrm{u}[3]^{\wedge} 3^{*} \mathrm{x} 0^{*} \mathrm{x} 3^{\wedge} 2-88152^{*} \mathrm{u}[3]^{\wedge} 3^{*} \mathrm{x} 3^{\wedge} 2^{*} \mathrm{x} 1-$ $125460^{*} \mathrm{u}[3]^{*} \mathrm{x} 0{ }^{*} \mathrm{x} 22^{\wedge} 2-43344^{*} \mathrm{u}[3]^{*} \mathrm{x} 1^{*} \mathrm{x} 2^{\wedge} 2+49956^{*} \mathrm{u}[3]^{*} \mathrm{x} 0^{*} \mathrm{x} 3^{\wedge} 2+35544^{*} \mathrm{u}[3]^{*} \mathrm{x} 3{ }^{\wedge} 2^{*} \mathrm{x} 1-$ $231594^{*} \mathrm{u}[3]^{*} \mathrm{x} 0^{\wedge} 2^{*} \mathrm{x} 2+53396^{*} \mathrm{u}[3]^{*} \mathrm{x} 00^{\wedge} 2^{*} \mathrm{x} 3+120080^{*} \mathrm{u}[3]^{*} \mathrm{x} 1^{\wedge} 2^{*} \mathrm{x} 2-$ $124584^{*} \mathrm{u}[3]^{*} \mathrm{x} 1^{\wedge} 2^{*} \mathrm{x} 3+29232^{*} \mathrm{x} 3^{*} \mathrm{y} 2^{*} \mathrm{x} 0+66146^{*} \mathrm{u}[3]^{\wedge} 5^{*} \mathrm{x} 0^{\wedge} 2^{*} \mathrm{x} 2+$ $430896^{*} \mathrm{u}[3]^{\wedge} 5^{*} \mathrm{x} 11^{\wedge} 2^{*} \mathrm{x} 2-55188^{*} \mathrm{u}[3]^{\wedge} 5^{*} \mathrm{x} 0{ }^{\wedge} 2^{*} \mathrm{x} 3-110536^{*} \mathrm{u}[3]^{\wedge} 5^{*} \mathrm{x} 1^{\wedge} 2^{*} \mathrm{x} 3+$ $199368^{*} \mathrm{u}[3]^{\wedge} 2^{*} \mathrm{x} 00^{\wedge} 2^{*} \mathrm{x} 2-73632^{*} \mathrm{u}[3]^{\wedge} 2^{*} \mathrm{x} 1^{\wedge} 2^{*} \mathrm{x} 2+21996^{*} \mathrm{u}[3]^{\wedge} 2^{*} \mathrm{x} 0^{\wedge} 2^{*} \mathrm{x} 3-$ $54444^{*} \mathrm{u}[3]^{\wedge} 2^{*} \mathrm{x} 11^{\wedge} 2^{*} \mathrm{x} 3-396188^{*} \mathrm{u}[3]^{\wedge} 2^{*} \mathrm{x} 0{ }^{*} \mathrm{x} 2{ }^{\wedge} 2+149240^{*} \mathrm{u}[3]^{\wedge} 2^{*} \mathrm{x} 00^{*} \mathrm{x} 3{ }^{\wedge} 2+$ $47008^{*} \mathrm{u}[3]^{\wedge} 2^{*} \mathrm{x} 1^{*} \mathrm{x} 22^{\wedge} 2-301834^{*} \mathrm{u}[3]^{\wedge} 2^{*} \mathrm{x} 3^{\wedge} 2^{*} \mathrm{x} 1+21504^{*} \mathrm{u}[3]^{\wedge} 2^{*} \mathrm{x} 2^{\wedge} 2^{*} \mathrm{y} 2+$ $152592^{*} \mathrm{u}[3]^{\wedge} 2^{*} \mathrm{x} 33^{\wedge} 2^{*} \mathrm{y} 2-30144^{*} \mathrm{u}[3]^{\wedge} 2^{*} \mathrm{x} 3{ }^{\wedge} 2^{*} \mathrm{y} 3+292224^{*} \mathrm{u}[3]^{\wedge} 2^{*} \mathrm{x} 22^{\wedge} 2^{*} \mathrm{y} 3-$ $154584^{*} \mathrm{u}[3]^{\wedge} 2^{*} \mathrm{y} 3^{*} \mathrm{x} 0{ }^{*} \mathrm{x} 3+38592^{*} \mathrm{u}[3]^{*} \mathrm{y} 00^{*} \mathrm{x} 1^{*} \mathrm{x} 3-8064^{*} \mathrm{u}[3]^{\wedge} 7^{*} \mathrm{y} 1^{\wedge} 2^{*} \mathrm{x} 3+$ $64512^{*} \mathrm{u}[3]^{\wedge} 7^{*} \mathrm{y} 0 \wedge 2^{*} \mathrm{x} 2+112896^{*} \mathrm{u}[3]^{\wedge} 7^{*} \mathrm{y} 0^{\wedge} 2^{*} \mathrm{x} 3-4608^{*} \mathrm{u}[3]^{\wedge} 7^{*} \mathrm{y} 1^{\wedge} 2^{*} \mathrm{x} 2+$ $60480^{*} \mathrm{u}[3]^{\wedge} 7^{*} \mathrm{y} 0^{*} \mathrm{x} 3^{\wedge} 2+25200^{*} \mathrm{u}[3]^{\wedge} 7^{*} \mathrm{y} 1^{*} \mathrm{x} 3^{\wedge} 2-684288^{*} \mathrm{u}[3]^{\wedge} 3^{*} \mathrm{y} 1^{*} \mathrm{x} 2^{\wedge} 2+$ $170360^{*} \mathrm{u}[3]^{\wedge} 2^{*} \mathrm{x} 0^{\wedge} 3-13896^{*} \mathrm{u}[3]^{\wedge} 7^{*} \mathrm{x} 0^{\wedge} 2^{*} \mathrm{x} 1+25200^{*} \mathrm{u}[3]^{\wedge} 7^{*} \mathrm{x} 0^{\wedge} 2^{*} \mathrm{y} 2-$ $19080^{*} \mathrm{u}[3]^{\wedge} 7^{*} \mathrm{x} 0 \wedge 2^{*} \mathrm{y} 3-226608^{*} \mathrm{u}[3]^{\wedge} 7^{*} \mathrm{x} 1^{\wedge} 2^{*} \mathrm{x} 0-128160^{*} \mathrm{u}[3]^{\wedge} 7^{*} \mathrm{y} 2^{*} \mathrm{x} 1^{\wedge} 2+$ $51840^{*} \mathrm{u}[3]^{\wedge} 7^{*} \mathrm{x} 1^{\wedge} 2^{*} \mathrm{y} 3-53880^{*} \mathrm{u}[3]^{\wedge} 5^{*} \mathrm{x} 0^{\wedge} 2^{*} \mathrm{x} 1+22896^{*} \mathrm{u}[3]^{\wedge} 5^{*} \mathrm{x} 0^{\wedge} 2^{*} \mathrm{y} 2+$ $139752^{*} \mathrm{u}[3]^{\wedge} 5^{*} \mathrm{x} 0^{\wedge} 2^{*} \mathrm{y} 3-11088^{*} \mathrm{u}[3]^{\wedge} 5^{*} \mathrm{x} 1^{\wedge} 2^{*} \mathrm{x} 0-88416^{*} \mathrm{u}[3]^{\wedge} 5^{*} \mathrm{y} 2^{*} \mathrm{x} 1^{\wedge} 2$ $41472^{*} \mathrm{u}[3]^{\wedge} 5^{*} \mathrm{x} 1^{\wedge} 2^{*} \mathrm{y} 3+28224^{*} \mathrm{u}[3]^{\wedge} 8^{*} \mathrm{y} 00^{*} \mathrm{y} 2^{*} \mathrm{x} 2-16128^{*} \mathrm{u}[3]^{\wedge} 8^{*} \mathrm{y} 0^{*} \mathrm{y} 3^{*} \mathrm{x} 2+$ $16128^{*} \mathrm{u}[3]^{\wedge} 8^{*} \mathrm{y} 1{ }^{*} \mathrm{y} 2^{*} \mathrm{x} 2-9216^{*} \mathrm{u}[3]^{\wedge} 8^{*} \mathrm{y} 1^{*} \mathrm{y} 3^{*} \mathrm{x} 2+54144^{*} \mathrm{u}[3]^{\wedge} 2^{*} \mathrm{y} 3{ }^{\wedge} 2^{*} \mathrm{x} 0+$ $225792^{*} \mathrm{u}[3]^{\wedge} 2^{*} \mathrm{y} 2^{\wedge} 2^{*} \mathrm{x} 1-24192^{*} \mathrm{u}[3]^{\wedge} 2^{*} \mathrm{y} 22^{\wedge} 2^{*} \mathrm{x} 0+13824^{*} \mathrm{u}[3]^{\wedge} 2^{*} \mathrm{y} 3^{\wedge} 2^{*} \mathrm{x} 1+$ $15888^{*} \mathrm{u}[3]^{\wedge} 2^{*} \mathrm{x} 0^{\wedge} 2^{*} \mathrm{y} 2-174528^{*} \mathrm{u}[3]^{\wedge} 2^{*} \mathrm{x} 0^{\wedge} 2^{*} \mathrm{y} 3+48384^{*} \mathrm{u}[3]^{\wedge} 2^{*} \mathrm{y} 2^{*} \mathrm{x} 1^{\wedge} 2-$ $136576^{*} \mathrm{u}[3]^{\wedge} 2^{*} \mathrm{x} 1^{\wedge} 2^{*} \mathrm{x} 0-15168^{*} \mathrm{u}[3]^{\wedge} 2^{*} \mathrm{x} 1{ }^{\wedge} 2^{*} \mathrm{y} 3+508024^{*} \mathrm{u}[3]^{\wedge} 2^{*} \mathrm{x} 0{ }^{\wedge} 2^{*} \mathrm{x} 1-$ $1152^{*} \mathrm{u}[3]^{\wedge} 3^{*} \mathrm{y} 1^{\wedge} 2^{*} \mathrm{x} 3+9216^{*} \mathrm{u}[3]^{\wedge} 3^{*} \mathrm{y} 0^{\wedge} 2^{*} \mathrm{x} 2-103680^{*} \mathrm{u}[3]^{\wedge} 3^{*} \mathrm{y} 0^{\wedge} 2^{*} \mathrm{x} 3-$ $69120^{*} \mathrm{u}[3]^{\wedge} 3^{*} \mathrm{y} 1^{\wedge} 2^{*} \mathrm{x} 2-150336^{*} \mathrm{u}[3]^{\wedge} 3^{*} \mathrm{y} 00^{*} \mathrm{x} 3^{\wedge} 2+17232^{*} \mathrm{u}[3]^{\wedge} 3^{*} \mathrm{y} 1^{*} \mathrm{x} 3^{\wedge} 2-$ $107136^{*} \mathrm{u}[3]^{\wedge} 5^{*} \mathrm{x} 0{ }^{\wedge} 2^{*} \mathrm{y} 0-159936^{*} \mathrm{u}[3]^{\wedge} 5^{*} \mathrm{y} 0^{*} \mathrm{x} 1^{\wedge} 2+173136^{*} \mathrm{u}[3]^{\wedge} 5^{*} \mathrm{x} 0^{\wedge} 2^{*} \mathrm{y} 1+$ $18432^{*} \mathrm{u}[3]^{\wedge} 5^{*} \mathrm{x} 11^{\wedge} 2^{*} \mathrm{y} 1+13440^{*} \mathrm{u}[3]^{\wedge} 7^{*} \mathrm{x} 00^{\wedge} 2^{*} \mathrm{y} 0-85824^{*} \mathrm{u}[3]^{\wedge} 7^{*} \mathrm{y} 00^{*} \mathrm{x} 1^{\wedge} 2-$ $161424^{*} \mathrm{u}[3]^{\wedge} 7^{*} \mathrm{x} 00^{\wedge} 2^{*} \mathrm{y} 1+12288^{*} \mathrm{u}[3]^{\wedge} 7^{*} \mathrm{x} 1^{\wedge} 2^{*} \mathrm{y} 1+19008^{*} \mathrm{y} 3^{*} \mathrm{y} 2^{*} \mathrm{x} 1+$ $334368^{*} \mathrm{u}[3]^{\wedge} 2^{*} \mathrm{y} 0 * \mathrm{y} 2^{*} \mathrm{x} 3-54144^{*} \mathrm{u}[3]^{\wedge} 2^{*} \mathrm{y} 00^{*} \mathrm{y} 3^{*} \mathrm{x} 3-13824^{*} \mathrm{u}[3]^{\wedge} 2^{*} \mathrm{y} 1{ }^{*} \mathrm{y} 3{ }^{*} \mathrm{x} 3-$ $5760^{*} \mathrm{u}[3]^{\wedge} 2^{*} \mathrm{y} 1{ }^{*} \mathrm{y} 2{ }^{*} \mathrm{x} 3-775680^{*} \mathrm{u}[3]^{\wedge} 3^{*} \mathrm{x} 00^{*} \mathrm{x} 1^{*} \mathrm{y} 0+89472^{*} \mathrm{u}[3]^{\wedge} 3^{*} \mathrm{x} 0{ }^{*} \mathrm{x} 1^{*} \mathrm{y} 1-$ $301680^{*} \mathrm{u}[3]^{\wedge} 2^{*} \mathrm{y} 0^{*} \mathrm{x} 3^{*} \mathrm{x} 2-241920^{*} \mathrm{u}[3]^{\wedge} 2^{*} \mathrm{y} 1^{*} \mathrm{x} 2^{*} \mathrm{x} 3+295680^{*} \mathrm{u}[3]^{\wedge} 5^{*} \mathrm{y} 1^{*} \mathrm{x} 2^{*} \mathrm{x} 3-$ $22392^{*} \mathrm{u}[3]^{\wedge} 5^{*} \mathrm{y} 1^{*} \mathrm{x} 1^{*} \mathrm{x} 3-326304^{*} \mathrm{u}[3]^{\wedge} 5^{*} \mathrm{y} 0^{*} \mathrm{x} 0^{*} \mathrm{x} 2-191808^{*} \mathrm{u}[3]^{\wedge} 5^{*} \mathrm{y} 0^{*} \mathrm{x} 1^{*} \mathrm{x} 2+$ $142848^{*} \mathrm{u}[3]^{\wedge} 5^{*} \mathrm{y} 1{ }^{*} \mathrm{x} 1{ }^{*} \mathrm{x} 2+215352^{*} \mathrm{u}[3]^{\wedge} 5{ }^{*} \mathrm{y} 1{ }^{*} \mathrm{x} 0{ }^{*} \mathrm{x} 2-75456^{*} \mathrm{u}[3]^{\wedge} 5^{*} \mathrm{y} 1{ }^{*} \mathrm{x} 0{ }^{*} \mathrm{x} 3-$ $480336 * \mathrm{u}[3]^{\wedge} 5 * \mathrm{y} 0 * \mathrm{x} 3{ }^{*} \mathrm{x} 2+551808^{*} \mathrm{u}[3]^{\wedge} 5 * \mathrm{y} 0{ }^{*} \mathrm{y} 1{ }^{*} \mathrm{x} 2+22176^{*} \mathrm{u}[3]^{\wedge} 5{ }^{*} \mathrm{y} 0 * \mathrm{y} 1{ }^{*} \mathrm{x} 3-$ $116136^{*} \mathrm{u}[3]^{\wedge} 5^{*} \mathrm{y} 0 * \mathrm{x} 00^{*} \mathrm{x} 3+261504^{*} \mathrm{u}[3]^{\wedge} 5^{*} \mathrm{y} 0^{*} \mathrm{x} 1{ }^{*} \mathrm{x} 3-39408^{*} \mathrm{u}[3]^{\wedge} 8^{*} \mathrm{x} 22^{\wedge} 2^{*} \mathrm{x} 3-$ $74892^{*} \mathrm{u}[3]^{\wedge} 8^{*} \mathrm{x} 3^{\wedge} 2^{*} \mathrm{x} 2-43008^{*} \mathrm{u}[3]^{\wedge} 8^{*} \mathrm{x} 2^{\wedge} 2^{*} \mathrm{y} 2+5880^{*} \mathrm{u}[3]^{\wedge} 8^{*} \mathrm{x} 3^{\wedge} 2^{*} \mathrm{y} 2+$ $24576^{*} \mathrm{u}[3]^{\wedge} 8^{*} \mathrm{x} 22^{\wedge} 2^{*} \mathrm{y} 3-3360^{*} \mathrm{u}[3]^{\wedge} 8^{*} \mathrm{x} 3^{\wedge} 2^{*} \mathrm{y} 3+39408^{*} \mathrm{x} 2^{\wedge} 2^{*} \mathrm{x} 3-74460^{*} \mathrm{x} 0{ }^{\wedge} 2^{*} \mathrm{x} 2-$ 
$6090^{*} \mathrm{x} 0{ }^{\wedge} 2^{*} \mathrm{x} 3+74096^{*} \mathrm{x} 1^{\wedge} 2^{*} \mathrm{x} 0-21216^{*} \mathrm{x} 1^{\wedge} 2^{*} \mathrm{y} 3-24576^{*} \mathrm{x} 2^{\wedge} 2^{*} \mathrm{y} 3+108868^{*} \mathrm{x} 0{ }^{\wedge} 2^{*} \mathrm{x} 1+$ $3072^{*} \mathrm{u}[3]^{\wedge} 8^{*} \mathrm{x} 2^{\wedge} 3+6090^{*} \mathrm{u}[3]^{\wedge} 8^{*} \mathrm{x} 33^{\wedge} 3+74016^{*} \mathrm{u}[3]^{*} \mathrm{y} 1^{*} \mathrm{y} 3^{*} \mathrm{x} 0+8064^{*} \mathrm{u}[3]^{*} \mathrm{y} 1^{*} \mathrm{y} 3^{*} \mathrm{x} 1-$ $9216^{*} \mathrm{u}[3]^{\wedge} 3^{*} \mathrm{y} 0^{*} \mathrm{y} 2^{*} \mathrm{x} 0-657792^{*} \mathrm{u}[3]^{\wedge} 3^{*} \mathrm{y} 0^{*} \mathrm{y} 2^{*} \mathrm{x} 1+103680^{*} \mathrm{u}[3]^{\wedge} 3^{*} \mathrm{y} 0{ }^{*} \mathrm{y} 3^{*} \mathrm{x} 0+$ $50688^{*} \mathrm{u}[3]^{\wedge} 3^{*} \mathrm{y} 0{ }^{*} \mathrm{y} 3^{*} \mathrm{x} 1+105984^{*} \mathrm{u}[3]^{\wedge} 3^{*} \mathrm{y} 1{ }^{*} \mathrm{y} 2{ }^{*} \mathrm{x} 0+69120^{*} \mathrm{u}[3]^{\wedge} 3^{*} \mathrm{y} 1^{*} \mathrm{y} 2^{*} \mathrm{x} 1-$ $72864^{*} \mathrm{u}[3]^{\wedge} 3^{*} \mathrm{y} 1^{*} \mathrm{y} 3^{*} \mathrm{x} 0+1152^{*} \mathrm{u}[3]^{\wedge} 3^{*} \mathrm{y} 1^{*} \mathrm{y} 3^{*} \mathrm{x} 1+9216^{*} \mathrm{y} 1^{*} \mathrm{y} 3^{*} \mathrm{x} 2+$ $104832^{*} \mathrm{u}[3]^{\wedge} 3^{*} \mathrm{y} 2^{\wedge} 2^{*} \mathrm{x} 3+59904^{*} \mathrm{u}[3]^{\wedge} 3^{*} \mathrm{y} 3^{\wedge} 2^{*} \mathrm{x} 2+22848^{*} \mathrm{u}[3]^{\wedge} 2^{*} \mathrm{y} 1^{*} \mathrm{x} 1{ }^{*} \mathrm{x} 3+$ $196608^{*} \mathrm{u}[3]^{\wedge} 2^{*} \mathrm{y} 0{ }^{*} \mathrm{x} 0 * \mathrm{x} 2+307392 * \mathrm{u}[3]^{\wedge} 2 * \mathrm{y} 0 * \mathrm{x} 1{ }^{*} \mathrm{x} 2-26880 * \mathrm{u}[3]^{\wedge} 2^{*} \mathrm{y} 1{ }^{*} \mathrm{x} 1{ }^{*} \mathrm{x} 2-$ $668544^{*} \mathrm{u}[3]^{\wedge} 2^{*} \mathrm{y} 1^{*} \mathrm{x} 0 * \mathrm{x} 2+136704^{*} \mathrm{u}[3]^{\wedge} 2^{*} \mathrm{y} 1{ }^{*} \mathrm{x} 00^{*} \mathrm{x} 3+144384^{*} \mathrm{u}[3]^{\wedge} 2^{*} \mathrm{y} 00^{*} \mathrm{x} 0{ }^{*} \mathrm{x} 3-$ $248304^{*} \mathrm{u}[3]^{\wedge} 2^{*} \mathrm{y} 0^{*} \mathrm{x} 11^{*} \mathrm{x} 3+239616^{*} \mathrm{u}[3]^{\wedge} 2^{*} \mathrm{y} 0{ }^{*} \mathrm{y} 1^{*} \mathrm{x} 0+29952^{*} \mathrm{u}[3]^{\wedge} 2^{*} \mathrm{y} 00^{*} \mathrm{y} 1{ }^{*} \mathrm{x} 1-$ $22848^{*} \mathrm{u}[3]^{\wedge} 6^{*} \mathrm{y} 1{ }^{*} \mathrm{x} 1{ }^{*} \mathrm{x} 3-196608^{*} \mathrm{u}[3]^{\wedge} 6^{*} \mathrm{y} 00^{*} \mathrm{x} 0{ }^{*} \mathrm{x} 2-307392^{*} \mathrm{u}[3]^{\wedge} 6^{*} \mathrm{y} 0{ }^{*} \mathrm{x} 1{ }^{*} \mathrm{x} 2+$ $26880^{*} \mathrm{u}[3]^{\wedge} 6^{*} \mathrm{y} 1{ }^{*} \mathrm{x} 1{ }^{*} \mathrm{x} 2+668544^{*} \mathrm{u}[3]^{\wedge} 6^{*} \mathrm{y} 1^{*} \mathrm{x} 00^{*} \mathrm{x} 2-136704^{*} \mathrm{u}[3]^{\wedge} 6^{*} \mathrm{y} 1^{*} \mathrm{x} 0{ }^{*} \mathrm{x} 3+$ $267264^{*} \mathrm{u}[3]^{\wedge} 4^{*} \mathrm{x} 3{ }^{*} \mathrm{y} 2{ }^{*} \mathrm{x} 0+451584^{*} \mathrm{u}[3]^{\wedge} 4{ }^{*} \mathrm{y} 2{ }^{*} \mathrm{x} 2{ }^{*} \mathrm{x} 1-288000^{*} \mathrm{u}[3]^{\wedge} 4^{*} \mathrm{y} 2{ }^{*} \mathrm{x} 1{ }^{*} \mathrm{x} 3-$ $429120^{*} \mathrm{u}[3]^{\wedge} 4^{*} \mathrm{x} 0^{*} \mathrm{y} 3^{*} \mathrm{x} 2+108288^{*} \mathrm{u}[3]^{\wedge} 4^{*} \mathrm{y} 3^{*} \mathrm{x} 0{ }^{*} \mathrm{x} 3-18432^{*} \mathrm{u}[3]^{\wedge} 4^{*} \mathrm{y} 3^{*} \mathrm{x} 1{ }^{*} \mathrm{x} 2+$ $27648^{*} \mathrm{u}[3]^{\wedge} 4^{*} \mathrm{x} 3^{*} \mathrm{y} 3^{*} \mathrm{x} 1-341640^{*} \mathrm{u}[3]^{\wedge} 6^{*} \mathrm{x} 0^{\wedge} 2^{*} \mathrm{x} 2-21996^{*} \mathrm{u}[3]^{\wedge} 6^{*} \mathrm{x} 0^{\wedge} 2^{*} \mathrm{x} 3+$ $433056^{*} \mathrm{u}[3]^{\wedge} 6^{*} \mathrm{x} 1^{\wedge} 2^{*} \mathrm{x} 2-125268^{*} \mathrm{u}[3]^{\wedge} 6^{*} \mathrm{x} 1^{\wedge} 2^{*} \mathrm{x} 3+594864^{*} \mathrm{u}[3]^{\wedge} 4^{*} \mathrm{x} 0^{*} \mathrm{x} 2^{\wedge} 2-$ $70848^{*} \mathrm{u}[3]^{\wedge} 4^{*} \mathrm{x} 1^{*} \mathrm{x} 2^{\wedge} 2-63216^{*} \mathrm{u}[3]^{\wedge} 4^{*} \mathrm{x} 0^{*} \mathrm{x} 3^{\wedge} 2+186912^{*} \mathrm{u}[3]^{\wedge} 4^{*} \mathrm{x} 3^{\wedge} 2^{*} \mathrm{x} 1-$ $67702^{*} \mathrm{u}[3]^{\wedge} 8^{*} \mathrm{x} 0^{*} \mathrm{x} 2^{\wedge} 2-7472^{*} \mathrm{u}[3]^{\wedge} 8^{*} \mathrm{x} 1^{*} \mathrm{x} 2^{\wedge} 2+7616^{*} \mathrm{u}[3]^{\wedge} 8^{*} \mathrm{x} 0^{*} \mathrm{x} 3^{\wedge} 2-$ $47843^{*} \mathrm{u}[3]^{\wedge} 8^{*} \mathrm{x} 3^{\wedge} 2^{*} \mathrm{x} 1-6090^{*} \mathrm{x} 3^{\wedge} 3-3072^{*} \mathrm{x} 22^{\wedge} 3+47843^{*} \mathrm{x} 3^{\wedge} 2^{*} \mathrm{x} 1+67702^{*} \mathrm{x} 00^{*} \mathrm{x} 2^{\wedge} 2+$ $66240^{*} \mathrm{u}[3]^{\wedge} 2^{*} \mathrm{y} 00^{*} \mathrm{x} 2^{\wedge} 2+60120^{*} \mathrm{u}[3]^{\wedge} 2^{*} \mathrm{y} 0 * \mathrm{x} 3^{\wedge} 2-31680^{*} \mathrm{u}[3]^{\wedge} 2^{*} \mathrm{y} 1^{*} \mathrm{x} 2^{\wedge} 2+$ $69120^{*} \mathrm{u}[3]^{\wedge} 2^{*} \mathrm{y} 1^{*} \mathrm{x} 3^{\wedge} 2-144384^{*} \mathrm{u}[3]^{\wedge} 6^{*} \mathrm{y} 0^{*} \mathrm{x} 0{ }^{*} \mathrm{x} 3+248304^{*} \mathrm{u}[3]^{\wedge} 6^{*} \mathrm{y} 0^{*} \mathrm{x} 1^{*} \mathrm{x} 3$;

\section{C.2 Right 3A-chain Constraint Variety}

The five Equations of the left constraint variety are as follows. The equations as presented are all set equal to zero. See Section 5.2.2. $\mathrm{u}[4]$ represent joint variable $u_{4}$.

$$
\begin{aligned}
& \mathrm{R} 1:=\mathrm{x} 0^{*} \mathrm{y} 0+\mathrm{x} 1^{*} \mathrm{y} 1+\mathrm{x} 2^{*} \mathrm{y} 2+\mathrm{x} 3^{*} \mathrm{y} 3 \\
& \mathrm{R} 2:=-1477063328881584^{*} \mathrm{u}[4]^{\wedge} 6^{*} \mathrm{x} 1^{*} \mathrm{x} 2+7220133090841200^{*} \mathrm{u}[4]^{\wedge} 4^{*} \mathrm{x} 1^{*} \mathrm{x} 2+ \\
& 7550500197974640^{*} \mathrm{u}[4]^{\wedge} 2^{*} \mathrm{x} 1^{*} \mathrm{x} 2+20647944195840^{*} \mathrm{u}[4]^{\wedge} 6^{*} \mathrm{y} 1^{*} \mathrm{x} 2- \\
& 103239720979200^{*} \mathrm{u}[4]^{\wedge} 4^{*} \mathrm{y} 1^{*} \mathrm{x} 2-103239720979200^{*} \mathrm{u}[4]^{\wedge} 2^{*} \mathrm{y} 1^{*} \mathrm{x} 2+ \\
& 82591776783360^{*} \mathrm{u}[4]^{\wedge} 5^{*} \mathrm{y} 1^{*} \mathrm{x} 1-82591776783360^{*} \mathrm{u}[4]^{*} \mathrm{y} 1^{*} \mathrm{x} 1+ \\
& 20647944195840^{*} \mathrm{y} 2^{*} \mathrm{x} 1^{*} \mathrm{u}[4]^{\wedge} 6-103239720979200^{*} \mathrm{y} 2^{*} \mathrm{x} 1^{*} \mathrm{u}[4]^{\wedge} 2- \\
& 82591776783360^{*} \mathrm{y} 2^{*} \mathrm{u}[4]^{\wedge} 5^{*} \mathrm{x} 2+82591776783360^{*} \mathrm{y} 2^{*} \mathrm{u}[4]^{*} \mathrm{x} 2- \\
& 103239720979200^{*} \mathrm{y} 2^{*} \mathrm{u}[4]^{\wedge} 4^{*} \mathrm{x} 1-3417551443233600^{*} \mathrm{x} 0^{*} \mathrm{u}[4]^{\wedge} 4^{*} \mathrm{x} 3+ \\
& 82591776783360^{*} \mathrm{x} 0^{*} \mathrm{u}[4]^{\wedge} 5^{*} \mathrm{y} 0+716546999360064^{*} \mathrm{u}[4]^{\wedge} 6^{*} \mathrm{x} 3^{*} \mathrm{x} 0- \\
& 20647944195840^{*} \mathrm{u}[4]^{\wedge} 6^{*} \mathrm{x} 3^{*} \mathrm{y} 0+103239720979200^{*} \mathrm{u}[4]^{\wedge} 4^{*} \mathrm{x} 3^{*} \mathrm{y} 0+ \\
& 103239720979200^{*} \mathrm{u}[4]^{\wedge} 2^{*} \mathrm{x} 3^{*} \mathrm{y} 0-20647944195840^{*} \mathrm{x} 0^{*} \mathrm{u}[4]^{\wedge} 6^{*} \mathrm{y} 3+ \\
& 103239720979200^{*} \mathrm{x} 0^{*} \mathrm{u}[4]^{\wedge} 2^{*} \mathrm{y} 3-3747918550367040^{*} \mathrm{x} 0^{*} \mathrm{x} 3^{*} \mathrm{u}[4]^{\wedge} 2- \\
& 82591776783360^{*} \mathrm{x} 0^{*} \mathrm{u}[4]^{*} \mathrm{y} 0+103239720979200^{*} \mathrm{x} 0^{*} \mathrm{y} 3^{*} \mathrm{u}[4]^{\wedge} 4- \\
& 82591776783360^{*} \mathrm{u}[4]^{\wedge} 5^{*} \mathrm{x} 3^{*} \mathrm{y} 3+82591776783360^{*} \mathrm{u}[4]^{*} \mathrm{x} 3^{*} \mathrm{y} 3+ \\
& 1412446054524288^{*} \mathrm{u}[4]^{\wedge} 5^{*} \mathrm{x} 3^{\wedge} 2+123887665175040^{*} \mathrm{u}[4]^{\wedge} 3^{*} \mathrm{x} 3^{\wedge} 2- \\
& 2974774601959008^{*} \mathrm{u}[4]^{*} \mathrm{x} 2^{\wedge} 2-1477063328881584^{*} \mathrm{x} 1^{*} \mathrm{x} 2+716546999360064^{*} \mathrm{x} 0^{*} \mathrm{x} 3+
\end{aligned}
$$


$445041213633120 * \mathrm{x} 0 * \mathrm{y} 0+11448668481120 * \mathrm{x} 22^{*} \mathrm{y} 2+825299378393880 * \mathrm{x} 33^{*} \mathrm{y} 3-$ $90908238741840{ }^{*} \mathrm{y} 2 * \mathrm{x} 3-1366634078806432^{*} \mathrm{x} 1{ }^{*} \mathrm{x} 3-126091545510606{ }^{*} \mathrm{x} 2 \wedge 2+$ $416102647605840^{*} \mathrm{y} 1 * \mathrm{x} 0+142992194676999 * \mathrm{x} 2 * \mathrm{x} 3-90908238741840 * \mathrm{x} 2 * \mathrm{y} 3-$ $2017022896534432 * \mathrm{x} 2 * \mathrm{x} 0+20647944195840 * \mathrm{y} 2 * \mathrm{x} 1+416102647605840 * \mathrm{y} 0 * \mathrm{x} 1+$ $676980718454751^{*} \mathrm{x} 0 * \mathrm{x} 1+391706833241880^{*} \mathrm{x} 1{ }^{*} \mathrm{y} 1-1344782653164426^{*} \mathrm{x} 0 \wedge 2+$ $398576174697051 * \mathrm{x} 3{ }^{\wedge} 2+20647944195840 * \mathrm{y} 1{ }^{*} \mathrm{x} 2+1699859059134231{ }^{*} \mathrm{x} 1 \wedge 2+$ $27530592261120 * \mathrm{x} 1 * \mathrm{y} 3+27530592261120^{*} \mathrm{y} 2 * \mathrm{x} 0+27530592261120 * \mathrm{x} 3{ }^{*} \mathrm{y} 1+$ $27530592261120 * \mathrm{x} 2 * \mathrm{y} 0-20647944195840 * \mathrm{x} 0 * \mathrm{y} 3-20647944195840 * \mathrm{x} 3{ }^{*} \mathrm{y} 0-$ $1344782653164426^{*} \mathrm{x} 0 \wedge 2^{*} \mathrm{u}[4]^{\wedge} 6+3699603681474150^{*} \mathrm{x} 0 \wedge 2^{*} \mathrm{u}[4]^{\wedge} 4+$ $2178571022431110^{*} \mathrm{x} 0^{\wedge} 2^{*} \mathrm{u}[4]^{\wedge} 2-1453741942915968^{*} \mathrm{u}[4]^{*} \mathrm{x} 33^{\wedge} 2+$ $27530592261120 * \mathrm{u}[4]^{\wedge} 6^{*} \mathrm{x} 2{ }^{*} \mathrm{y} 0+82591776783360 * \mathrm{u}[4]^{\wedge} 4{ }^{*} \mathrm{x} 2{ }^{*} \mathrm{y} 0+$ $82591776783360 * \mathrm{u}[4]^{\wedge} 2^{*} \mathrm{x} 2{ }^{*} \mathrm{y} 0+27530592261120^{*} \mathrm{x} 1{ }^{*} \mathrm{u}[4]^{\wedge} 6^{*} \mathrm{y} 3+$ $82591776783360^{*} \mathrm{x} 1^{*} \mathrm{u}[4]^{\wedge} 2^{*} \mathrm{y} 3+82591776783360 * \mathrm{x} 11^{*} \mathrm{y} 3 * \mathrm{u}[4]^{\wedge} 4+$ $1300777635456000^{*} \mathrm{u}[4]^{*} \mathrm{x} 2{ }^{*} \mathrm{x} 3-1300777635456000^{*} \mathrm{x} 1{ }^{*} \mathrm{u}[4]^{\wedge} 5^{*} \mathrm{x} 0-$ $123887665175040^{*} \mathrm{u}[4]^{\wedge} 3^{*} \mathrm{x} 1^{\wedge} 2+2974774601959008^{*} \mathrm{u}[4]^{*} \mathrm{x} 1^{\wedge} 2-$ $2933478713567328 * \mathrm{u}[4]^{\wedge} 5^{*} \mathrm{x} 1^{\wedge} 2+2933478713567328^{*} \mathrm{x} 2^{\wedge} 2^{*} \mathrm{u}[4]^{\wedge} 5+$ $123887665175040^{*} \times 2^{\wedge} 2^{*} \mathrm{u}[4]^{\wedge} 3-123887665175040^{*} \mathrm{x} 0^{\wedge} 2^{*} \mathrm{u}[4]^{\wedge} 3-$ $1412446054524288^{*} \mathrm{x} 0 \wedge 2^{*} \mathrm{u}[4]^{\wedge} 5+1453741942915968^{*} \mathrm{u}[4]^{*} \mathrm{x} 0 \wedge 2-$ $2549727132618747^{*} \mathrm{x} 0^{*} \mathrm{u}[4]^{\wedge} 4^{*} \mathrm{x} 1-1965140235127500^{*} \mathrm{x} 0^{*} \mathrm{u}[4]^{\wedge} 5^{*} \mathrm{x} 2+$ $1951166453184000^{*} \mathrm{x} 0 * \mathrm{u}[4]^{\wedge} 3^{*} \mathrm{x} 2+142992194676999^{*} \mathrm{u}[4]^{\wedge} 6^{*} \mathrm{x} 3^{*} \mathrm{x} 2+$ $27530592261120^{*} \mathrm{u}[4]^{\wedge} 6^{*} \mathrm{x} 0 * \mathrm{y} 2+82591776783360^{*} \mathrm{u}[4]^{\wedge} 4^{*} \mathrm{x} 00^{*} \mathrm{y} 2+$ $82591776783360 * \mathrm{u}[4]^{\wedge} 2^{*} \mathrm{x} 0 * \mathrm{y} 2+82591776783360^{*}{ }^{*} 3^{*} \mathrm{u}[4]^{\wedge} 4^{*} \mathrm{y} 1+$ $27530592261120^{*} \mathrm{x} 3{ }^{*} \mathrm{y} 1^{*} \mathrm{u}[4]^{\wedge} 6+82591776783360^{*} \mathrm{x} 33^{*} \mathrm{y} 1{ }^{*} \mathrm{u}[4]^{\wedge} 2-$ $4151692703952003^{*} \mathrm{u}[4]^{\wedge} 4^{*} \mathrm{x} 3^{*} \mathrm{x} 2-1550137433040003^{*} \mathrm{u}[4]^{\wedge} 2^{*} \mathrm{x} 3^{*} \mathrm{x} 2+$ $676980718454751^{*} \mathrm{x} 0 * \mathrm{u}[4]^{\wedge} 6^{*} \mathrm{x} 1+51828138293253^{*} \mathrm{x} 0 * \mathrm{u}[4]^{\wedge} 2^{*} \mathrm{x} 1+$ $1314751417399500^{*} \mathrm{x} 00^{*} \mathrm{u}[4]^{*} \mathrm{x} 2+1965140235127500^{*} \mathrm{u}[4]^{\wedge} 5^{*} \mathrm{x} 3^{*} \mathrm{x} 1-$ $1951166453184000^{*} \mathrm{u}[4]^{\wedge} 3^{*} \mathrm{x} 3^{*} \mathrm{x} 1-1314751417399500^{*} \mathrm{u}[4]^{*} \mathrm{x} 3{ }^{*} \mathrm{x} 1-$ $7353693476206668^{*} \mathrm{x} 00^{*} \mathrm{u}[4]^{\wedge} 5^{*} \mathrm{x} 3+2281548988564560^{*} \mathrm{x} 0 * \mathrm{u}[4]^{\wedge} 3^{*} \mathrm{x} 3+$ $2856156299942400^{*} \mathrm{x} 0 * \mathrm{u}[4]^{\wedge} 4^{*} \mathrm{y} 0-760516329521520^{*} \mathrm{x} 0 * \mathrm{u}[4]^{\wedge} 5^{*} \mathrm{y} 3+$ $825299378393880^{*} \mathrm{u}[4]^{\wedge} 6^{*} \mathrm{x} 3^{*} \mathrm{y} 3+398576174697051^{*} \mathrm{u}[4]^{\wedge} 6^{*} \mathrm{x} 3{ }^{\wedge} 2$ $6538223116876275^{*} \mathrm{u}[4]^{\wedge} 4^{*} \mathrm{x} 3^{\wedge} 2-5017190457833235^{*} \mathrm{u}[4]^{\wedge} 2^{*} \mathrm{x} 3^{\wedge} 2$ $90908238741840^{*} \mathrm{u}[4]^{\wedge} 6^{*} \mathrm{y} 3^{*} \mathrm{x} 2-1573502351681520^{*} \mathrm{u}[4]^{\wedge} 4^{*} \mathrm{y} 3^{*} \mathrm{x} 2-$ $1573502351681520^{*} \mathrm{u}[4]^{\wedge} 2^{*} \mathrm{y} 3^{*} \mathrm{x} 2+416102647605840^{*} \mathrm{y} 0 * \mathrm{u}[4]^{\wedge} 6^{*} \mathrm{x} 1-$ $52469692638480 *^{*} 0^{*} \mathrm{u}[4]^{\wedge} 2{ }^{*} \mathrm{x} 1+65038881772800 *^{*} \mathrm{y} 0 * \mathrm{u}[4]{ }^{*} \mathrm{x} 2+$ $650388817728000^{*} \mathrm{u}[4]^{\wedge} 5^{*} \mathrm{y} 3^{*} \mathrm{x} 1-650388817728000^{*} \mathrm{u}[4]^{*} \mathrm{y} 3^{*} \mathrm{x} 1-$ $52469692638480^{*} \mathrm{y} 1^{*} \mathrm{u}[4]^{\wedge} 4^{*} \mathrm{x} 0+650388817728000^{*} \mathrm{y} 1^{*} \mathrm{u}[4]^{\wedge} 5^{*} \mathrm{x} 3-$ $1366634078806432^{*} \mathrm{x} 1{ }^{*} \mathrm{u}[4]^{\wedge} 6^{*} \mathrm{x} 3-6701457507331296^{*} \mathrm{x} 1^{*} \mathrm{u}[4]^{\wedge} 2^{*} \mathrm{x} 3+$ $1300777635456000^{*} \mathrm{x} 1{ }^{*} \mathrm{u}[4]^{*} \mathrm{x} 0-1300777635456000^{*} \mathrm{u}[4]^{\wedge} 5^{*} \mathrm{x} 2{ }^{*} \mathrm{x} 3-$ $90908238741840^{*} \mathrm{u}[4]^{\wedge} 6 * \mathrm{y} 2{ }^{*} \mathrm{x} 3-1573502351681520^{*} \mathrm{u}[4]^{\wedge} 4^{*} \mathrm{y} 2{ }^{*} \mathrm{x} 3-$ $1573502351681520^{*} u[4]^{\wedge} 2^{*} \mathrm{y} 2^{*} \mathrm{x} 3+416102647605840^{*} \mathrm{y} 1^{*} \mathrm{u}[4]^{\wedge} 6^{*} \mathrm{x} 0-$ $52469692638480^{*} \mathrm{y} 1^{*} \mathrm{u}[4]^{\wedge} 2^{*} \mathrm{x} 0-650388817728000^{*} \mathrm{y} 1^{*} \mathrm{u}[4]^{*} \mathrm{x} 3-$ $650388817728000^{*} \mathrm{u}[4]^{\wedge} 5^{*} \mathrm{y} 2{ }^{*} \mathrm{x} 0+650388817728000^{*} \mathrm{u}[4]^{*} \mathrm{y} 2{ }^{*} \mathrm{x} 0-$ $6701457507331296^{*} \mathrm{x} 1^{*} \mathrm{u}[4]^{\wedge} 4^{*} \mathrm{x} 3-2017022896534432^{*} \mathrm{u}[4]^{\wedge} 6^{*} \mathrm{x} 2^{*} \mathrm{x} 0$ $3449513418691296^{*} \mathrm{u}[4]^{\wedge} 4^{*} \mathrm{x} 2{ }^{*} \mathrm{x} 0-3449513418691296^{*} \mathrm{u}[4]^{\wedge} 2^{*} \mathrm{x} 2^{*} \mathrm{x} 0+$ $1699859059134231^{*} \mathrm{x} 1^{\wedge} 2^{*} \mathrm{u}[4]^{\wedge} 6-2964741570698175^{*} \mathrm{x} 1^{\wedge} 2^{*} \mathrm{u}[4]^{\wedge} 4-$ 
$1443708911655135^{*} \mathrm{x} 1^{\wedge} 2^{*} \mathrm{u}[4]^{\wedge} 2+954865476138600^{*} \mathrm{u}[4]^{\wedge} 4^{*} \mathrm{x} 3^{*} \mathrm{y} 3+$ $954865476138600^{*} \mathrm{u}[4]^{\wedge} 2^{*} \mathrm{x} 33^{*} \mathrm{y} 3+445041213633120^{*} \mathrm{x} 0{ }^{*} \mathrm{u}[4]^{\wedge} 6^{*} \mathrm{y} 0+$ $2856156299942400^{*} \mathrm{x} 0 * \mathrm{u}[4]^{\wedge} 2^{*} \mathrm{y} 0+6593177146685148 * \mathrm{x} 0 * \mathrm{u}[4]^{*} \mathrm{x} 3+$ $760516329521520^{*} \mathrm{x} 0 * \mathrm{u}[4]^{*} \mathrm{y} 3-760516329521520^{*} \mathrm{u}[4]^{\wedge} 5^{*} \mathrm{x} 3{ }^{*} \mathrm{y} 0+$ $760516329521520 * \mathrm{u}[4] * \mathrm{x} 3 * \mathrm{y} 0-7684060583340108 * \mathrm{x} 1{ }^{*} \mathrm{u}[4]^{\wedge} 5{ }^{*} \mathrm{x} 2+$ $2281548988564560^{*} \mathrm{x} 1{ }^{*} \mathrm{u}[4]^{\wedge} 3^{*} \mathrm{x} 2-345912159317400^{*} \mathrm{x} 1^{*} \mathrm{u}[4]^{\wedge} 4^{*} \mathrm{y} 1-$ $760516329521520^{*} \mathrm{x} 1^{*} \mathrm{u}[4]^{\wedge} 5^{*} \mathrm{y} 2-126091545510606^{*} \mathrm{u}[4]^{\wedge} 6^{*} \mathrm{x} 2^{\wedge} 2+$ $7686044111569050^{*} \mathrm{u}[4]^{\wedge} 4^{*} \mathrm{x} 22^{\wedge} 2+6165011452526010^{*} \mathrm{u}[4]^{\wedge} 2^{*} \mathrm{x} 22^{\wedge} 2+$ $11448668481120 * \mathrm{u}[4]^{\wedge} 6^{*} \mathrm{x} 2 * \mathrm{y} 2+1555378664486400 * \mathrm{u}[4]^{\wedge} 4 * \mathrm{x} 2 * \mathrm{y} 2+$ $1555378664486400^{*} \mathrm{u}[4]^{\wedge} 2^{*} \mathrm{x} 2^{*} \mathrm{y} 2+391706833241880^{*} \mathrm{x} 1{ }^{*} \mathrm{u}[4]^{\wedge} 6^{*} \mathrm{y} 1-$ $345912159317400^{*} \mathrm{x} 1{ }^{*} \mathrm{u}[4]^{\wedge} 2^{*} \mathrm{y} 1+6923544253818588^{*} \mathrm{x} 1{ }^{*} \mathrm{u}[4]^{*} \mathrm{x} 2+$ $760516329521520{ }^{*} \mathrm{x} 1 *_{\mathrm{u}}[4]^{*} \mathrm{y} 2-760516329521520 * \mathrm{u}[4]^{\wedge} 5^{*} \mathrm{x} 2{ }^{*} \mathrm{y} 1+$ $760516329521520^{*} \mathrm{u}[4]^{*} \mathrm{x} 2{ }^{*} \mathrm{y} 1-52469692638480^{*} \mathrm{y} 0 * \mathrm{u}[4]^{\wedge} 4^{*} \mathrm{x} 1-$ $650388817728000^{*} \mathrm{y} 0^{*} \mathrm{u}[4]^{\wedge} 5^{*} \mathrm{x} 2$;

$\mathrm{R} 3:=-52943446656^{*} \mathrm{u}[4]^{\wedge} 4^{*} \mathrm{x} 1{ }^{*} \mathrm{x} 2+317660679936^{*} \mathrm{u}[4]^{\wedge} 2^{*} \mathrm{x} 1{ }^{*} \mathrm{x} 2+$ $52943446656^{*} \mathrm{x} 0^{*} \mathrm{u}[4]^{\wedge} 4^{*} \mathrm{x} 3-317660679936^{*} \mathrm{x} 0^{*} \mathrm{x} 3^{*} \mathrm{u}[4]^{\wedge} 2+105886893312^{*} \mathrm{u}[4]^{\wedge} 3^{*} \mathrm{x} 3^{\wedge} 2-$ $105886893312 * \mathrm{u}[4] * \mathrm{x} 2 \wedge 2-52943446656 * \mathrm{x} 1{ }^{*} \mathrm{x} 2+52943446656 * \mathrm{x} 0 * \mathrm{x} 3-$ $70591262208^{*} \mathrm{x} 1^{*} \mathrm{x} 3+521699173021^{*} \mathrm{x} 2{ }^{\wedge} 2+233098048056^{*} \mathrm{x} 2{ }^{*} \mathrm{x} 3-70591262208^{*} \mathrm{x} 2{ }^{*} \mathrm{x} 0-$ $1066929865656^{*} \mathrm{x} 0 * \mathrm{x} 1-34188705379{ }^{*} \mathrm{x} 0{ }^{\wedge} 2-521699173021^{*} \mathrm{x} 3{ }^{\wedge} 2+34188705379{ }^{*} \mathrm{x} 1{ }^{\wedge} 2-$ $34188705379^{*} \mathrm{x} 0^{\wedge} 2^{*} \mathrm{u}[4]^{\wedge} 4-2018419281326^{*} \mathrm{x} 0^{\wedge} 2^{*} \mathrm{u}[4]^{\wedge} 2-105886893312^{*} \mathrm{u}[4]^{*} \mathrm{x} 3^{\wedge} 2-$ $105886893312^{*} \mathrm{u}[4]^{\wedge} 3^{*} \mathrm{x} 1^{\wedge} 2+105886893312^{*} \mathrm{u}[4]^{*} \mathrm{x} 1^{\wedge} 2+105886893312^{*} \mathrm{x} 2^{\wedge} 2^{*} \mathrm{u}[4]^{\wedge} 3-$ $105886893312^{*} \mathrm{x} 0^{\wedge} 2^{*} \mathrm{u}[4]^{\wedge} 3+105886893312^{*} \mathrm{u}[4]^{*} \mathrm{x} 0^{\wedge} 2-1066929865656^{*} \mathrm{x} 0 * \mathrm{u}[4]^{\wedge} 4^{*} \mathrm{x} 1+$ $1667663635200^{*} \mathrm{x} 0^{*} \mathrm{u}[4]^{\wedge} 3^{*} \mathrm{x} 2+233098048056^{*} \mathrm{u}[4]^{\wedge} 4^{*} \mathrm{x} 3^{*} \mathrm{x} 2+$ $3801523366512^{*} \mathrm{u}[4]^{\wedge} 2^{*} \mathrm{x} 3^{*} \mathrm{x} 2+1201467539088^{*} \mathrm{x} 00^{*} \mathrm{u}[4]^{\wedge} 2^{*} \mathrm{x} 1-1667663635200^{*} \mathrm{x} 0{ }^{*} \mathrm{u}[4]^{*} \mathrm{x} 2$ $-1667663635200^{*} \mathrm{u}[4]^{\wedge} 3^{*} \mathrm{x} 3^{*} \mathrm{x} 1+1667663635200^{*} \mathrm{u}[4]^{*} \mathrm{x} 3^{*} \mathrm{x} 1+$ $1950041870568{ }^{*} \mathrm{x} 0 * \mathrm{u}[4]^{\wedge} 3^{*} \mathrm{x} 3-521699173021^{*} \mathrm{u}[4]^{\wedge} 4^{*} \mathrm{x} 3^{\wedge} 2+906643524526^{*} \mathrm{u}[4]^{\wedge} 2^{*} \mathrm{x} 3^{\wedge} 2-$ $141182524416^{*} \mathrm{x} 1^{*} \mathrm{u}[4]^{\wedge} 2^{*} \mathrm{x} 3-70591262208^{*} \mathrm{x} 1^{*} \mathrm{u}[4]^{\wedge} 4^{*} \mathrm{x} 3-70591262208^{*} \mathrm{u}[4]^{\wedge} 4^{*} \mathrm{x} 2^{*} \mathrm{x} 0-$ $141182524416^{*} \mathrm{u}[4]^{\wedge} 2^{*} \mathrm{x} 2^{*} \mathrm{x} 0+34188705379^{*} \mathrm{x} 1^{\wedge} 2^{*} \mathrm{u}[4]^{\wedge} 4+2018419281326^{*} \mathrm{x} 1^{\wedge} 2^{*} \mathrm{u}[4]^{\wedge} 2$ $1950041870568^{*} \mathrm{x} 0^{*} \mathrm{u}[4]^{*} \mathrm{x} 3+1950041870568^{*} \mathrm{x} 1{ }^{*} \mathrm{u}[4]^{\wedge} 3^{*} \mathrm{x} 2+521699173021^{*} \mathrm{u}[4]^{\wedge} 4^{*} \mathrm{x} 22^{\wedge} 2-$ $906643524526^{*} \mathrm{u}[4]^{\wedge} 2^{*} \mathrm{x} 2{ }^{\wedge} 2-1950041870568^{*} \mathrm{x} 1{ }^{*} \mathrm{u}[4]^{*} \mathrm{x} 2$

$\mathrm{R} 4:=-156946966504740432^{*} \mathrm{u}[4]^{\wedge} 6^{*} \mathrm{x} 1^{*} \mathrm{x} 2+783082996988034960^{*} \mathrm{u}[4]^{\wedge} 4^{*} \mathrm{x} 1{ }^{*} \mathrm{x} 2+$ $786386668059369360^{*} \mathrm{u}[4]^{\wedge} 2^{*} \mathrm{x} 1{ }^{*} \mathrm{x} 2-2933973168921600 * \mathrm{u}[4]^{\wedge} 6{ }^{*} \mathrm{y} 1{ }^{*} \mathrm{x} 2-$ $10453755042432000^{*} \mathrm{u}[4]^{\wedge} 4^{*} \mathrm{y} 1{ }^{*} \mathrm{x} 2-10453755042432000^{*} \mathrm{u}[4]^{\wedge} 2^{*} \mathrm{y} 1{ }^{*} \mathrm{x} 2+$ $825917767833600^{*} \mathrm{u}[4]^{\wedge} 5^{*} \mathrm{y} 1^{*} \mathrm{x} 1-825917767833600^{*} \mathrm{u}[4]^{*} \mathrm{y} 1^{*} \mathrm{x} 1+$ $3346932052838400 * \mathrm{y} 2{ }^{*} \mathrm{x} 1{ }^{*} \mathrm{u}[4]^{\wedge} 6+8388960622848000^{*} \mathrm{y} 2{ }^{*} \mathrm{x} 1{ }^{*} \mathrm{u}[4]^{\wedge} 2-$ $825917767833600^{*} \mathrm{y} 2^{*} \mathrm{u}[4]^{\wedge} 5^{*} \mathrm{x} 2+825917767833600^{*} \mathrm{y} 2^{*} \mathrm{u}[4]^{*} \mathrm{x} 2+$ $8388960622848000 *^{*} 2^{*} \mathrm{u}[4]^{\wedge} 4{ }^{*} \mathrm{x} 1+641492774970473040 *^{*} 00^{*} \mathrm{u}[4]^{\wedge} 4{ }^{*} \mathrm{x} 3+$ $825917767833600^{*} \mathrm{x} 00^{*} \mathrm{u}[4]^{\wedge} 5^{*} \mathrm{y} 0-127968187886961168^{*} \mathrm{u}[4]^{\wedge} 6^{*} \mathrm{x} 3^{*} \mathrm{x} 0+$ $10576555447161600^{*} \mathrm{u}[4]^{\wedge} 6^{*} \mathrm{x} 3^{*} \mathrm{y} 0+33381501877152000^{*} \mathrm{u}[4]^{\wedge} 4^{*} \mathrm{x} 3^{*} \mathrm{y} 0+$ $33381501877152000^{*} \mathrm{u}[4]^{\wedge} 2^{*} \mathrm{x} 3^{*} \mathrm{y} 0-10989514331078400^{*} \mathrm{x} 0 * \mathrm{u}[4]^{\wedge} 6^{*} \mathrm{y} 3-$ $31316707457568000^{*} \mathrm{x} 0 * \mathrm{u}[4]^{\wedge} 2^{*} \mathrm{y} 3+638189103899138640 * \mathrm{x} 0 * \mathrm{x} 3{ }^{*} \mathrm{u}[4]^{\wedge} 2-$ $825917767833600 * \mathrm{x} 0 * \mathrm{u}[4]^{*} \mathrm{y} 0-31316707457568000 * \mathrm{x} 0 * \mathrm{y} 3 * \mathrm{u}[4]^{\wedge} 4-$ $825917767833600^{*} \mathrm{u}[4]^{\wedge} 5^{*} \mathrm{x} 3{ }^{*} \mathrm{y} 3+825917767833600^{*} \mathrm{u}[4]^{*} \mathrm{x} 3{ }^{*} \mathrm{y} 3-$ $245359820326760736^{*} \mathrm{u}[4]^{\wedge} 5^{*} \mathrm{x} 3^{\wedge} 2+22804946429990400^{*} \mathrm{u}[4]^{\wedge} 3^{*} \mathrm{x} 3^{\wedge} 2$ - 
$310959959840559264 * \mathrm{u}[4]{ }^{*} \mathrm{x} 22^{\wedge} 2-156946966504740432{ }^{*} \mathrm{x} 1{ }^{*} \mathrm{x} 2-$

$127968187886961168 * \mathrm{x} 0 * \mathrm{x} 3+4450412136331200 * \mathrm{x} 0 * \mathrm{y} 0+114486684811200 * \mathrm{x} 2 * \mathrm{y} 2-$

$3213112500000000^{*} \mathrm{u}[4]^{\wedge} 5^{*} \mathrm{x} 22^{*} \mathrm{y} 3+3213112500000000^{*} \mathrm{u}[4]^{*} \mathrm{x} 2{ }^{*} \mathrm{y} 3+$

$8252993783938800 * \mathrm{x} 3 * \mathrm{y} 3-975967375734000 * \mathrm{y} 2 * \mathrm{x} 3+4016390625000000 * \mathrm{y} 2 \wedge 2+$

$28783408940237024^{*} \mathrm{x} 1{ }^{*} \mathrm{x} 3+77585031833873837^{*} \mathrm{x} 22^{\wedge} 2-27903213535626000^{*} \mathrm{y} 1{ }^{*} \mathrm{x} 0+$

$138654995548243200^{*} \mathrm{x} 1{ }^{*} \mathrm{u}[4]^{\wedge} 3^{*} \mathrm{x} 0-58669989974721218^{*} \mathrm{x} 2{ }^{*} \mathrm{x} 3-842197399102800^{*} \mathrm{x} 2{ }^{*} \mathrm{y} 3$

- $138654995548243200^{*} \mathrm{u}[4]^{\wedge} 3^{*} \mathrm{x} 2{ }^{*} \mathrm{x} 3-8290808350002976^{*} \mathrm{x} 2{ }^{*} \mathrm{x} 0+$

$3346932052838400^{*} \mathrm{y} 2 * \mathrm{x} 1+36225266487742800^{*} \mathrm{y} 0 * \mathrm{x} 1+63123766233735518^{*} \mathrm{x} 0 * \mathrm{x} 1+$

$4016390625000000^{*} \mathrm{y} 3^{\wedge} 2+3917068332418800^{*} \mathrm{x} 1 * \mathrm{y} 1+133454129804564437^{*} \mathrm{x} 0 \wedge 2+$

$79518231731757013^{*} \mathrm{x} 3^{\wedge} 2+12049171875000000^{*} \mathrm{u}[4]^{\wedge} 2^{*} \mathrm{y} 1^{\wedge} 2-2933973168921600^{*} \mathrm{y} 1{ }^{*} \mathrm{x} 2$

$+152464471622375213^{*} \mathrm{x} 1^{\wedge} 2-16065562500000000^{*} \mathrm{u}[4]^{\wedge} 3^{*} \mathrm{x} 0 * \mathrm{y} 3+$

$4016390625000000^{*} \mathrm{y} 1^{\wedge} 2+4016390625000000^{*} \mathrm{y} 0^{\wedge} 2+12049171875000000^{*} \mathrm{u}[4]^{\wedge} 2^{*} \mathrm{y} 0 \wedge 2+$ $12049171875000000^{*} \mathrm{u}[4]^{\wedge} 4^{*} \mathrm{y} 0^{\wedge} 2+4016390625000000^{*} \mathrm{u}[4]^{\wedge} 6^{*} \mathrm{y} 0^{\wedge} 2+$ $12049171875000000^{*} \mathrm{y} 3^{\wedge} 2^{*} \mathrm{u}[4]^{\wedge} 4+4016390625000000^{*} \mathrm{y} 3^{\wedge} 2^{*} \mathrm{u}[4]^{\wedge} 6+$ $12049171875000000^{*} \mathrm{y} 3^{\wedge} 2^{*} \mathrm{u}[4]^{\wedge} 2+16065562500000000^{*} \mathrm{x} 3^{*} \mathrm{u}[4]^{\wedge} 3^{*} \mathrm{y} 0-$ $33585164839449600 * \mathrm{x} 1{ }^{*} \mathrm{y} 3+35742332934672000^{*} \mathrm{y} 2 * \mathrm{x} 0+34135776684672000^{*} \mathrm{x} 3{ }^{*} \mathrm{y} 1-$ $35191721089449600 * \mathrm{x} 2 * \mathrm{y} 0-10989514331078400 * \mathrm{x} 0 * \mathrm{y} 3+10576555447161600 * \mathrm{x} 3 * \mathrm{y} 0-$ $3213112500000000^{*} \mathrm{x} 1^{*} \mathrm{u}[4]^{\wedge} 5^{*} \mathrm{y} 0+3213112500000000^{*} \mathrm{x} 1^{*} \mathrm{u}[4]^{*} \mathrm{y} 0+$ $133454129804564437^{*} \mathrm{x} 0 \wedge 2^{*} \mathrm{u}[4]^{\wedge} 6+196240351042678815^{*} \mathrm{x} 0 \wedge 2^{*} \mathrm{u}[4]^{\wedge} 4+$ $181030024452248415^{*} \mathrm{x} 0{ }^{\wedge} 2^{*} \mathrm{u}[4]^{\wedge} 2+12049171875000000^{*} \mathrm{y}^{\wedge} 2^{*} \mathrm{u}[4]^{\wedge} 4+$ $4016390625000000^{*} \mathrm{y} 2^{\wedge} 2^{*} \mathrm{u}[4]^{\wedge} 6+12049171875000000^{*} \mathrm{y} 2^{\wedge} 2^{*} \mathrm{u}[4]^{\wedge} 2+$ $266512931221083936 * \mathrm{u}[4]^{*} \mathrm{x} 33^{\wedge} 2-35191721089449600 * \mathrm{u}[4]^{\wedge} 6^{*} \mathrm{x} 2{ }^{*} \mathrm{y} 0$ $99148938268348800^{*} \mathrm{u}[4]^{\wedge} 4^{*} \mathrm{x} 2{ }^{*} \mathrm{y} 0-99148938268348800^{*} \mathrm{u}[4]^{\wedge} 2^{*} \mathrm{x} 2{ }^{*} \mathrm{y} 0-$ $33585164839449600^{*} \mathrm{x} 1{ }^{*} \mathrm{u}[4]^{\wedge} 6^{*} \mathrm{y} 3-107181719518348800^{*} \mathrm{x} 1{ }^{*} \mathrm{u}[4]^{\wedge} 2^{*} \mathrm{y} 3-$ $107181719518348800 *^{*} 1^{*} \mathrm{y} 3 * \mathrm{u}[4]^{\wedge} 4+4820936806358400^{*} \mathrm{u}[4]^{*} \mathrm{x} 2{ }^{*} \mathrm{x} 3-$ $4820936806358400^{*} \mathrm{x} 1^{*} \mathrm{u}[4]^{\wedge} 5^{*} \mathrm{x} 0+5042028570009600^{*} \mathrm{u}[4]^{\wedge} 3^{*} \mathrm{x} 1^{\wedge} 2+$ $317240865062319264^{*} \mathrm{u}[4]^{*} \mathrm{x} 1^{\wedge} 2-310547000956642464^{*} \mathrm{u}[4]^{\wedge} 5^{*} \mathrm{x} 1^{\wedge} 2+$ $316827906178402464^{*} \times 2^{\wedge} 2^{*} \mathrm{u}[4]^{\wedge} 5+7519781873510400^{*} \times 2^{\wedge} 2^{*} \mathrm{u}[4]^{\wedge} 3+$ $20327193126489600^{*} \mathrm{x} 0^{\wedge} 2^{*} \mathrm{u}[4]^{\wedge} 3+266925890105000736^{*} \mathrm{x} 0^{\wedge} 2^{*} \mathrm{u}[4]^{\wedge} 5-$ $244946861442843936^{*} \mathrm{u}[4]^{*} \mathrm{x} 0^{\wedge} 2+158548417310589354^{*} \mathrm{x} 00^{*} \mathrm{u}[4]^{\wedge} 4^{*} \mathrm{x} 1+$ $19837858416700200^{*} \mathrm{x} 00^{*} \mathrm{u}[4]^{\wedge} 5 * \mathrm{x} 2+83506374578577600^{*} \mathrm{x} 00^{*} \mathrm{u}[4]^{\wedge} 3^{*} \mathrm{x} 2-$ $58669989974721218^{*} \mathrm{u}[4]^{\wedge} 6^{*} \mathrm{x} 3^{*} \mathrm{x} 2-16065562500000000^{*} \mathrm{x} 22^{*} \mathrm{u}[4]^{\wedge} 3^{*} \mathrm{y} 1+$ $16065562500000000^{*} \mathrm{u}[4]^{\wedge} 3^{*} \mathrm{x} 1{ }^{*} \mathrm{y} 2+35742332934672000^{*} \mathrm{u}[4]^{\wedge} 6^{*} \mathrm{x} 0 * \mathrm{y} 2+$ $100800773804016000^{*} \mathrm{u}[4]^{\wedge} 4^{*} \mathrm{x} 0 * \mathrm{y} 2+100800773804016000 * \mathrm{u}[4]^{\wedge} 2^{*} \mathrm{x} 00^{*} \mathrm{y} 2+$ $108833555054016000^{*} \mathrm{x} 3^{*} \mathrm{u}[4]^{\wedge} 4^{*} \mathrm{y} 1+34135776684672000^{*} \mathrm{x} 3^{*} \mathrm{y} 1^{*} \mathrm{u}[4]^{\wedge} 6+$ $108833555054016000^{*} \mathrm{x} 3^{*} \mathrm{y} 1{ }^{*} \mathrm{u}[4]^{\wedge} 2-206832851314780854^{*} \mathrm{u}[4]^{\wedge} 4^{*} \mathrm{x} 3^{*} \mathrm{x} 2-$ $180817298605660854^{*} \mathrm{u}[4]^{\wedge} 2^{*} \mathrm{x} 3^{*} \mathrm{x} 2+63123766233735518^{*} \mathrm{x} 0^{*} \mathrm{u}[4]^{\wedge} 6^{*} \mathrm{x} 1+$ $184563970019709354^{*} \mathrm{x} 00^{*} \mathrm{u}[4]^{\wedge} 2^{*} \mathrm{x} 1+37652963452757400^{*} \mathrm{x} 00^{*} \mathrm{u}[4]^{*} \mathrm{x} 2+$ $44156851630037400^{*} \mathrm{u}[4]^{\wedge} 5^{*} \mathrm{x} 3^{*} \mathrm{x} 1+44483045514897600^{*} \mathrm{u}[4]^{\wedge} 3^{*} \mathrm{x} 3^{*} \mathrm{x} 1+$ $26341746593980200^{*} \mathrm{u}[4]^{*} \mathrm{x} 3^{*} \mathrm{x} 1+211941010643622096^{*} \mathrm{x} 0^{*} \mathrm{u}[4]^{\wedge} 5^{*} \mathrm{x} 3+$ $22815489885645600^{*} \mathrm{x} 0 * \mathrm{u}[4]^{\wedge} 3^{*} \mathrm{x} 3+28561562999424000^{*} \mathrm{x} 0 * \mathrm{u}[4]^{\wedge} 4^{*} \mathrm{y} 0-$ $15637944545215200^{*} \mathrm{x} 0{ }^{*} \mathrm{u}[4]^{\wedge} 5^{*} \mathrm{y} 3+8252993783938800 * \mathrm{u}[4]^{\wedge} 6^{*} \mathrm{x} 3{ }^{*} \mathrm{y} 3+$ $79518231731757013^{*} \mathrm{u}[4]^{\wedge} 6^{*} \mathrm{x} 3^{\wedge} 2+450709514816285535^{*} \mathrm{u}[4]^{\wedge} 4^{*} \mathrm{x} 3^{\wedge} 2+$ $465919841406715935^{*} \mathrm{u}[4]^{\wedge} 2^{*} \mathrm{x} 3^{\wedge} 2-3213112500000000^{*} \mathrm{u}[4]^{*} \mathrm{x} 0 * \mathrm{y} 1+$ $3213112500000000^{*} \mathrm{x} 3{ }^{*} \mathrm{u}[4]^{\wedge} 5{ }^{*} \mathrm{y} 2-3213112500000000^{*} \mathrm{x} 3{ }^{*} \mathrm{u}[4]^{*} \mathrm{y} 2+$ 
$3213112500000000 * \mathrm{u}[4]^{\wedge} 5{ }^{*} \mathrm{x} 0 *^{*} \mathrm{y} 1+12049171875000000^{*} \mathrm{u}[4]^{\wedge} 4{ }^{*} \mathrm{y} 1 \wedge 2+$ $4016390625000000^{*} \mathrm{u}[4]^{\wedge} 6^{*} \mathrm{y} 1^{\wedge} 2-842197399102800^{*} \mathrm{u}[4]^{\wedge} 6^{*} \mathrm{y} 3^{*} \mathrm{x} 2-$

$144058868551868400^{*} \mathrm{u}[4]^{\wedge} 4^{*} \mathrm{y} 3^{*} \mathrm{x} 2-144058868551868400^{*} \mathrm{u}[4]^{\wedge} 2^{*} \mathrm{y} 3^{*} \mathrm{x} 2+$ $36225266487742800^{*} \mathrm{y} 0^{*} \mathrm{u}[4]^{\wedge} 6^{*} \mathrm{x} 1-32856476891331600^{*} \mathrm{y} 0 * \mathrm{u}[4]^{\wedge} 2^{*} \mathrm{x} 1+$ $70766138177280000^{*} \mathrm{y} 0 * \mathrm{u}[4]^{*} \mathrm{x} 2+70766138177280000^{*} \mathrm{u}[4]^{\wedge} 5^{*} \mathrm{y} 3^{*} \mathrm{x} 1-$ $70766138177280000 * \mathrm{u}[4]^{*} \mathrm{y} 3{ }^{*} \mathrm{x} 1+31807083038562000^{*} \mathrm{y} 1{ }^{*} \mathrm{u}[4]^{\wedge} 4^{*} \mathrm{x} 0-$ $57758361822720000^{*} \mathrm{y} 1^{*} \mathrm{u}[4]^{\wedge} 5^{*} \mathrm{x} 3+28783408940237024^{*} \mathrm{x} 1{ }^{*} \mathrm{u}[4]^{\wedge} 6{ }^{*} \mathrm{x} 3-$ $61946642340248928^{*} \mathrm{x} 1{ }^{*} \mathrm{u}[4]^{\wedge} 2^{*} \mathrm{x} 3+143475932354601600^{*} \mathrm{x} 1{ }^{*} \mathrm{u}[4]^{*} \mathrm{x} 0-$ $143475932354601600^{*} \mathrm{u}[4]^{\wedge} 5^{*} \mathrm{x} 2{ }^{*} \mathrm{x} 3-975967375734000^{*} \mathrm{u}[4]^{\wedge} 6^{*} \mathrm{y} 2{ }^{*} \mathrm{x} 3+$ $112588821518238000^{*} \mathrm{u}[4]^{\wedge} 4^{*} \mathrm{y} 2^{*} \mathrm{x} 3+112588821518238000^{*} \mathrm{u}[4]^{\wedge} 2^{*} \mathrm{y} 2^{*} \mathrm{x} 3-$ $27903213535626000^{*} \mathrm{y} 1^{*} \mathrm{u}[4]^{\wedge} 6^{*} \mathrm{x} 0+31807083038562000^{*} \mathrm{y} 1^{*} \mathrm{u}[4]^{\wedge} 2^{*} \mathrm{x} 0+$ $57758361822720000^{*} \mathrm{y} 1^{*} \mathrm{u}[4]^{*} \mathrm{x} 3+57758361822720000^{*} \mathrm{u}[4]^{\wedge} 5^{*} \mathrm{y} 2^{*} \mathrm{x} 0-$ $57758361822720000 * \mathrm{u}[4]^{*} \mathrm{y} 2{ }^{*} \mathrm{x} 0-61946642340248928{ }^{*} \mathrm{x} 1{ }^{*} \mathrm{u}[4]^{\wedge} 4{ }^{*} \mathrm{x} 3-$ $8290808350002976^{*} \mathrm{u}[4]^{\wedge} 6^{*} \mathrm{x} 22^{*} \mathrm{x} 0+123424444110951072^{*} \mathrm{u}[4]^{\wedge} 4^{*} \mathrm{x} 2{ }^{*} \mathrm{x} 0+$ $123424444110951072^{*} \mathrm{u}[4]^{\wedge} 2^{*} \mathrm{x} 2^{*} \mathrm{x} 0+152464471622375213^{*} \mathrm{x} 1^{\wedge} 2^{*} \mathrm{u}[4]^{\wedge} 6+$ $154286883042904935^{*} \mathrm{x} 1^{\wedge} 2^{*} \mathrm{u}[4]^{\wedge} 4+169497209633335335^{*} \mathrm{x} 1^{\wedge} 2^{*} \mathrm{u}[4]^{\wedge} 2+$ $9548654761386000^{*} \mathrm{u}[4]^{\wedge} 4^{*} \mathrm{x} 3{ }^{*} \mathrm{y} 3+9548654761386000^{*} \mathrm{u}[4]^{\wedge} 2^{*} \mathrm{x} 3{ }^{*} \mathrm{y} 3+$ $4450412136331200^{*} \mathrm{x} 0^{*} \mathrm{u}[4]^{\wedge} 6^{*} \mathrm{y} 0+28561562999424000^{*} \mathrm{x} 0^{*} \mathrm{u}[4]^{\wedge} 2^{*} \mathrm{y} 0-$ $219546173938837296^{*} \mathrm{x} 0{ }^{*} \mathrm{u}[4]^{*} \mathrm{x} 3-427617954784800^{*} \mathrm{x} 0 * \mathrm{u}[4]{ }^{*} \mathrm{y} 3+$ $427617954784800^{*} \mathrm{u}[4]^{\wedge} 5^{*} \mathrm{x} 3^{*} \mathrm{y} 0+15637944545215200^{*} \mathrm{u}[4]^{*} \mathrm{x} 3^{*} \mathrm{y} 0-$ $303320340801613104^{*} \mathrm{x} 1^{*} \mathrm{u}[4]^{\wedge} 5^{*} \mathrm{x} 2+22815489885645600^{*} \mathrm{x} 1{ }^{*} \mathrm{u}[4]^{\wedge} 3^{*} \mathrm{x} 2-$ $3459121593174000^{*} \mathrm{x} 1^{*} \mathrm{u}[4]^{\wedge} 4^{*} \mathrm{y} 1+427617954784800^{*} \mathrm{x} 1^{*} \mathrm{u}[4]^{\wedge} 5^{*} \mathrm{y} 2+$ $77585031833873837^{*} \mathrm{u}[4]^{\wedge} 6^{*} \mathrm{x} 2^{\wedge} 2+543894408575842215^{*} \mathrm{u}[4]^{\wedge} 4^{*} \mathrm{x} 2^{\wedge} 2+$ $528684081985411815^{*} \mathrm{u}[4]^{\wedge} 2^{*} \mathrm{x} 2^{\wedge} 2+114486684811200^{*} \mathrm{u}[4]^{\wedge} 6^{*} \mathrm{x} 2^{*} \mathrm{y} 2+$ $15553786644864000^{*} \mathrm{u}[4]^{\wedge} 4^{*} \mathrm{x} 2^{*} \mathrm{y} 2+15553786644864000^{*} \mathrm{u}[4]^{\wedge} 2^{*} \mathrm{x} 2^{*} \mathrm{y} 2+$ $3917068332418800^{*} \mathrm{x} 1{ }^{*} \mathrm{u}[4]^{\wedge} 6^{*} \mathrm{y} 1-3459121593174000^{*} \mathrm{x} 1{ }^{*} \mathrm{u}[4]^{\wedge} 2^{*} \mathrm{y} 1+$ $295715177506397904^{*} \mathrm{x} 1^{*} \mathrm{u}[4]^{*} \mathrm{x} 2+15637944545215200^{*} \mathrm{x} 1^{*} \mathrm{u}[4]^{*} \mathrm{y} 2-$ $15637944545215200^{*} \mathrm{u}[4]^{\wedge} 5^{*} \mathrm{x} 2{ }^{*} \mathrm{y} 1-427617954784800^{*} \mathrm{u}[4]^{*} \mathrm{x} 2{ }^{*} \mathrm{y} 1-$ $32856476891331600^{*} \mathrm{y} 0^{*} \mathrm{u}[4]^{\wedge} 4^{*} \mathrm{x} 1-70766138177280000^{*} \mathrm{y} 0 * \mathrm{u}[4]^{\wedge} 5^{*} \mathrm{x} 2$;

$\mathrm{R} 5:=291378868314391437120^{*} \mathrm{y} 1{ }^{*} \mathrm{x} 22^{\wedge}+1127944258631582745600^{*} \mathrm{y} 22^{*} \mathrm{x} 0-$ $1057455480818056320000^{*} \mathrm{y} 3^{\wedge} 2^{*} \mathrm{x} 0+1091060905699028160000^{*} \mathrm{y} 2^{\wedge} 2^{*} \mathrm{x} 1-$ $991482478184634508800^{*} \mathrm{y} 3^{\wedge} 2^{*} \mathrm{x} 1+5401844184114386012160^{*} \mathrm{x} 2^{\wedge} 2^{*} \mathrm{y} 2+$ $55056924943281160197120^{*} \mathrm{x} 3^{\wedge} 2^{*} \mathrm{y} 2+5598387771274662174720^{*} \mathrm{x} 3^{\wedge} 2^{*} \mathrm{y} 3+$ $18174492306614674346469{ }^{*} \mathrm{x} 1 \wedge 2^{*} \mathrm{x} 2+25617269986436514350880^{*} \mathrm{x} 00^{\wedge} 2^{*} \mathrm{y} 2-$ $21711208940383930674468^{*} \mathrm{x} 1^{\wedge} 2^{*} \mathrm{x} 3+4711635870444400402624^{*} \mathrm{x} 3^{\wedge} 2^{*} \mathrm{x} 2$ $17338230409252052851200^{*} \mathrm{u}[4]^{\wedge} 7^{*} \mathrm{y} 3{ }^{*} \mathrm{y} 2 * \mathrm{x} 2+$ $16774320186743841446400^{*} \mathrm{u}[4]^{\wedge} 5^{*} \mathrm{y} 3^{*} \mathrm{y} 2{ }^{*} \mathrm{x} 2+$ $16774320186743841446400^{*} \mathrm{u}[4]^{\wedge} 3^{*} \mathrm{y} 3^{*} \mathrm{y} 2^{*} \mathrm{x} 2-43624465016040787259520^{*} \mathrm{y} 0^{*} \mathrm{u}[4]^{\wedge} 2^{*} \mathrm{x} 2^{\wedge} 2-$ $1279279299508405292160 * \mathrm{y} 0 * \mathrm{x} 2 \wedge 2-190565470287648518400 * \mathrm{y} 0 * \mathrm{y} 2 * \mathrm{x} 0+$ $405063993724924060800^{*} \mathrm{y} 0{ }^{*} \mathrm{y} 2{ }^{*} \mathrm{x} 1+1752642622838867760000^{*} \mathrm{y} 0{ }^{*} \mathrm{y} 3^{*} \mathrm{x} 0+$ $1703378537844060916800^{*} \mathrm{y} 0 * \mathrm{y} 3{ }^{*} \mathrm{x} 1+1479691023253837804800^{*} \mathrm{u}[4]^{\wedge} 3^{*} \mathrm{y} 1{ }^{\wedge} 2^{*} \mathrm{x} 0+$ $3235493335334612601600^{*} \mathrm{y} 1^{\wedge} 2^{*} \mathrm{u}[4]^{*} \mathrm{x} 0+9036164713779368755200^{*} \mathrm{y} 2^{\wedge} 2^{*} \mathrm{u}[4]^{\wedge} 5^{*} \mathrm{x} 0-$ $5292190581022112601600^{*} \mathrm{y} 2^{\wedge} 2^{*} \mathrm{u}[4]^{\wedge} 6^{*} \mathrm{x} 3+269791910343122918400^{*} \mathrm{y} 2^{\wedge} 2^{*} \mathrm{u}[4]^{\wedge} 7^{*} \mathrm{x} 0+$ $9036164713779368755200^{*} \mathrm{y} 2^{\wedge} 2^{*} \mathrm{u}[4]^{\wedge} 3^{*} \mathrm{x} 0+1479691023253837804800^{*} \mathrm{u}[4]^{\wedge} 5^{*} \mathrm{y} 11^{\wedge} 2^{*} \mathrm{x} 0+$ $1786905335344377081600^{*} \mathrm{u}[4]^{\wedge} 6^{*} \mathrm{y} 1^{\wedge} 2^{*} \mathrm{x} 3+3235493335334612601600^{*} \mathrm{u}[4]^{\wedge} 7^{*} \mathrm{y} 1^{\wedge} 2^{*} \mathrm{x} 0-$ 
$1786905335344377081600^{*} \mathrm{u}[4]^{\wedge} 2^{*} \mathrm{y} 1 \wedge 2^{*} \mathrm{x} 3-6340662535971952849920^{*} \mathrm{y} 3^{*} \mathrm{x} 0{ }^{*} \mathrm{x} 3-$ $3753845339908216860960{ }^{*} \mathrm{y} 1{ }^{*} \mathrm{x} 2 * \mathrm{x} 3-3194124073124161396320^{*} \mathrm{y} 3{ }^{*} \mathrm{x} 1{ }^{*} \mathrm{x} 2+$ $14800386780734781657600^{*} \mathrm{u}[4]^{\wedge} 7^{*} \mathrm{y} 1{ }^{*} \mathrm{y} 2{ }^{*} \mathrm{x} 0-9797059463257609171200 * \mathrm{u}[4]^{\wedge} 8{ }^{*} \mathrm{y} 1{ }^{*} \mathrm{y} 2{ }^{*} \mathrm{x} 3$ $+49194892487984781657600 * \mathrm{u}[4]^{\wedge} 6 * \mathrm{y} 1{ }^{*} \mathrm{y} 2{ }^{*} \mathrm{x} 3-$

$93176862486795655027200^{*} \mathrm{u}[4]^{\wedge} 3^{*} \mathrm{y} 1^{*} \mathrm{y} 2^{*} \mathrm{x} 0-49194892487984781657600^{*} \mathrm{u}[4]^{\wedge} 2^{*} \mathrm{y} 1{ }^{*} \mathrm{y} 2^{*} \mathrm{x} 3$ $-33132832235538884266464^{*} \mathrm{x} 1^{\wedge} 3+82198370111755628009952^{*} \mathrm{x} 0^{\wedge} 3-$ $2299110435146754927720^{*} \mathrm{y} 1{ }^{*} \mathrm{x} 1{ }^{*} \mathrm{x} 3-5252665183180681291200^{*} \mathrm{y} 00^{*} \mathrm{x} 0{ }^{*} \mathrm{x} 2+$ $1840808357045006846760 * \mathrm{y} 0 * \mathrm{x} 1 * \mathrm{x} 2+206890164254980442206127 * \mathrm{x} 3{ }^{*} \mathrm{x} 0 * \mathrm{x} 1+$ $264748635488702047680^{*} \mathrm{y} 1{ }^{*} \mathrm{x} 1{ }^{*} \mathrm{x} 2-2020240807451666342861{ }^{*} \mathrm{x} 1{ }^{*} \mathrm{x} 2{ }^{*} \mathrm{x} 0+$ $65404861482304852019280 * \mathrm{y} 2 * \mathrm{x} 0 * \mathrm{x} 1-3775203587071902492720 * \mathrm{y} 1 * \mathrm{x} 0 * \mathrm{x} 2-$ $23400136985728511842200 * \mathrm{y} 3 * \mathrm{x} 1 * \mathrm{x} 0+32821252489809599144760 * \mathrm{y} 1{ }^{*} \mathrm{x} 0{ }^{*} \mathrm{x} 3-$ $1786905335344377081600^{*} \mathrm{y} 1^{*} \mathrm{y} 3^{*} \mathrm{x} 1{ }^{*} \mathrm{u}[4]^{\wedge} 6+1786905335344377081600^{*} \mathrm{y} 1^{*} \mathrm{y} 3^{*} \mathrm{x} 1{ }^{*} \mathrm{u}[4]^{\wedge} 2$ $+1163244578015311459200^{*} \mathrm{y} 1^{*} \mathrm{y} 3^{*} \mathrm{x} 1^{*} \mathrm{u}[4]^{\wedge} 8-$

$77798182871245394794320^{*} \mathrm{u}[4]^{\wedge} 6^{*} \mathrm{x} 2^{*} \mathrm{y} 0^{*} \mathrm{x} 1-405063993724924060800^{*} \mathrm{u}[4]^{\wedge} 8^{*} \mathrm{y} 2^{*} \mathrm{y} 0 * \mathrm{x} 1-$ $19843341199245149469840 * \mathrm{u}[4]^{\wedge} 5^{*} \mathrm{x} 2{ }^{*} \mathrm{y} 3^{*} \mathrm{x} 1+42796597038355204243920 * \mathrm{u}[4]^{*} \mathrm{x} 2{ }^{*} \mathrm{y} 3{ }^{*} \mathrm{x} 1$ $1840808357045006846760^{*} \mathrm{u}[4]^{\wedge} 8^{*} \mathrm{x} 2^{*} \mathrm{y} 0^{*} \mathrm{x} 1-10522175115517020633600^{*} \mathrm{u}[4]^{\wedge} 4^{*} \mathrm{x} 2{ }^{*} \mathrm{y} 0{ }^{*} \mathrm{x} 1$ $+83729585721228374160720^{*} \mathrm{u}[4]^{\wedge} 2^{*} \mathrm{x} 22^{*} \mathrm{y} 0 * \mathrm{x} 1-$ $3303266503925151878400 * \mathrm{u}[4]^{\wedge} 2^{*} \mathrm{y} 2 * \mathrm{y} 0 * \mathrm{x} 1+10274195902536578188800^{*} \mathrm{y} 0 * \mathrm{y} 2 * \mathrm{x} 3+$ $1057455480818056320000^{*} \mathrm{y} 0 * \mathrm{y} 3 * \mathrm{x} 3+991482478184634508800^{*} \mathrm{y} 1{ }^{*} \mathrm{y} 3{ }^{*} \mathrm{x} 3+$ $9797059463257609171200 * \mathrm{y} 1 * \mathrm{y} 2 * \mathrm{x} 3-1127944258631582745600 * \mathrm{y} 0 * \mathrm{y} 2 * \mathrm{x} 2-$ $15399091795240857600^{*} \mathrm{y} 0 * \mathrm{y} 3^{*} \mathrm{x} 2-1091060905699028160000^{*} \mathrm{y} 1 * \mathrm{y} 2 * \mathrm{x} 2-$ $50452392170760206904000^{*} \mathrm{u}[4]^{\wedge} 5^{*} \mathrm{y} 3{ }^{*} \mathrm{x} 2{ }^{*} \mathrm{x} 3-59478194981719957636800^{*} \mathrm{u}[4]^{\wedge} 3^{*} \mathrm{y} 3^{*} \mathrm{x} 2{ }^{*} \mathrm{x} 3$ - $14557743444423855249600^{*} \mathrm{y} 0 * \mathrm{u}[4]^{\wedge} 7^{*} \mathrm{x} 1^{*} \mathrm{x} 3-$ $42969719198551456584000^{*} \mathrm{y} 0 * \mathrm{u}[4]^{\wedge} 5^{*} \mathrm{x} 1{ }^{*} \mathrm{x} 3-29831680774535874811200^{*} \mathrm{y} 0 * \mathrm{u}[4]^{\wedge} 3^{*} \mathrm{x} 1{ }^{*} \mathrm{x} 3$ $+54831809398272126432000^{*} \mathrm{y} 0{ }^{*} \mathrm{u}[4]^{\wedge} 2^{*} \mathrm{x} 2{ }^{*} \mathrm{x} 3+57299306881059208650252^{*} \mathrm{x} 3{ }^{*} \mathrm{x} 2{ }^{*} \mathrm{x} 1-$ $5983861486203974529108^{*} \mathrm{x} 1^{*} \mathrm{x} 22^{\wedge} 2+2079544508933558882400^{*} \mathrm{x} 33^{*} \mathrm{y} 3^{*} \mathrm{x} 1-$ $4346601035912351825760 * \mathrm{y} 2{ }^{*} \mathrm{x} 2 * \mathrm{x} 1-37921673816254342260720^{*} \mathrm{x} 0{ }^{\wedge} 2^{*} \mathrm{y} 3+$ $135578848495050008763840^{*} \mathrm{u}[4]^{\wedge} 5^{*} \mathrm{y} 2{ }^{*} \mathrm{x} 0^{\wedge} 2+79709289395814544619520^{*} \mathrm{u}[4]^{\wedge} 7^{*} \mathrm{y} 2^{*} \mathrm{x} 3^{\wedge} 2$ $+149084402445532683426240^{*} \mathrm{u}[4]^{\wedge} 3^{*} \mathrm{y} 2^{*} \mathrm{x} 0^{\wedge} 2-$ $101646051294037196067840^{*} \mathrm{u}[4]^{\wedge} 5^{*} \mathrm{y} 2^{*} \mathrm{x} 3^{\wedge} 2-61569425182846209405120^{*} \mathrm{u}[4]^{*} \mathrm{y} 2^{*} \mathrm{x} 0 \wedge{ }^{\wedge} 2$ $74085619817964039690240 * \mathrm{u}[4]^{\wedge} 3^{*} \mathrm{y} 2{ }^{*} \mathrm{x} 3^{\wedge} 2-180046878560083717974720^{*} \mathrm{y} 1{ }^{*} \mathrm{u}[4]^{\wedge} 2^{*} \mathrm{x} 3^{\wedge} 2$ $287180868756038331620160^{*} \mathrm{u}[4]^{\wedge} 6^{*} \mathrm{y} 2^{*} \mathrm{x} 0 * \mathrm{x} 3+$ $10579655873680963430400^{*} \mathrm{u}[4]^{\wedge} 44^{*} \mathrm{y} 2 * \mathrm{x} 0 * \mathrm{x} 3+$ $283736445287719295050560^{*} \mathrm{u}[4]^{\wedge} 2^{*} \mathrm{y} 2 * \mathrm{x} 0 * \mathrm{x} 3+$ $105658607245681579325760 * \mathrm{y} 1{ }^{*} \mathrm{x} 0 * \mathrm{u}[4]^{*} \mathrm{x} 3+12525421186940126750400 * \mathrm{y} 0 * \mathrm{x} 3{ }^{*} \mathrm{x} 2+$ $42226305546474056255040 * \mathrm{y} 2 * \mathrm{x} 1{ }^{*} \mathrm{x} 3-10258796810741337331200 * \mathrm{y} 3 *^{*} \mathrm{y} 2{ }^{*} \mathrm{x} 0-$ $3209475719724647443200^{*} \mathrm{y} 1^{*} \mathrm{y} 3^{*} \mathrm{x} 0{ }^{*} \mathrm{u}[4]^{\wedge} 6+3209475719724647443200^{*} \mathrm{y} 1^{*} \mathrm{y} 3^{*} \mathrm{x} 0{ }^{*} \mathrm{u}[4]^{\wedge} 2$ $+1300727151043926278400^{*} \mathrm{y} 1^{*} \mathrm{y} 3^{*} \mathrm{x} 0 * \mathrm{u}[4]^{\wedge} 8+190565470287648518400^{*} \mathrm{u}[4]^{\wedge} 8^{*} \mathrm{y} 2{ }^{*} \mathrm{y} 0 * \mathrm{x} 0$ $+5657046035049273335040^{*} \mathrm{x} 2{ }^{*} \mathrm{y} 2{ }^{*} \mathrm{x} 0+203100113433600924121044^{*} \mathrm{x} 0^{*} \mathrm{x} 3^{\wedge} 2+$ $1752642622838867760000^{*} \mathrm{y}^{\wedge} 2^{*} \mathrm{u}[4]^{\wedge} 8^{*} \mathrm{x} 3+3505285245677735520000^{*} \mathrm{y} 0^{\wedge} 2^{*} \mathrm{u}[4]^{\wedge} 6^{*} \mathrm{x} 3-$ $3505285245677735520000^{*} \mathrm{u}[4]^{\wedge} 2^{*} \mathrm{y} 0^{\wedge} 2^{*} \mathrm{x} 3+3505285245677735520000^{*} \mathrm{y} 0 * \mathrm{y} 3^{*} \mathrm{u}[4]^{*} \mathrm{x} 3+$ $3505285245677735520000^{*} \mathrm{y} 0 * \mathrm{u}[4]^{\wedge} 2^{*} \mathrm{y} 3^{*} \mathrm{x} 0-6884805159238901424960^{*} \mathrm{y} 0 * \mathrm{x} 3^{\wedge} 2+$ $8812925434587974919840 * \mathrm{y} 1{ }^{*} \mathrm{x} 3{ }^{\wedge} 2-228905006250211834745964{ }^{*} \mathrm{x} 2{ }^{*} \mathrm{x} 0{ }^{*} \mathrm{x} 3-$ $5110425547932466257600^{*} \mathrm{x} 0 * \mathrm{y} 3^{*} \mathrm{x} 2+991482478184634508800^{*} \mathrm{y} 3^{\wedge} 2^{*} \mathrm{u}[4]^{\wedge} 8^{*} \mathrm{x} 1+$ $1523054879130730982400^{*} \mathrm{y} 3^{\wedge} 2^{*} \mathrm{u}[4]^{\wedge} 2^{*} \mathrm{x} 1+137804289328617516739056^{*} \mathrm{u}[4]^{\wedge} 2^{*} \mathrm{x} 3^{\wedge} 2^{*} \mathrm{x} 1-$ 
$2079544508933558882400^{*} \mathrm{u}[4]^{\wedge} 8^{*} \mathrm{y} 3^{*} \mathrm{x} 1{ }^{*} \mathrm{x} 3+5732553133718924126400^{*} \mathrm{u}[4]^{\wedge} 6^{*} \mathrm{y} 3{ }^{*} \mathrm{x} 1{ }^{*} \mathrm{x} 3$ $+3842737178376611174400 * \mathrm{u}[4]^{\wedge} 4 * \mathrm{y} 3 * \mathrm{x} 1 * \mathrm{x} 3-$

$333606977071976182901760^{*} \mathrm{x} 22^{\wedge} 2^{*} \mathrm{u}[4]^{\wedge} 5^{*} \mathrm{y} 1+1043879265474888960000^{*} \mathrm{u}[4]^{\wedge} 4^{*} \mathrm{x} 11^{\wedge} 2^{*} \mathrm{y} 2-$ $10535160913620090168960{ }^{*} \mathrm{x} 22^{\wedge} 2^{*} \mathrm{u}[4]^{\wedge} 7^{*} \mathrm{y} 1+260969816368722240000^{*} \mathrm{u}[4]^{\wedge} 6^{*} \mathrm{y} 1^{\wedge} 2^{*} \mathrm{x} 2+$ $43089308478092662025760^{*} \mathrm{x} 1^{\wedge} 2^{*} \mathrm{u}[4]^{\wedge} 5^{*} \mathrm{y} 1+65878607026449415769760^{*} \mathrm{x} 1^{\wedge} 2^{*} \mathrm{u}[4]^{\wedge} 6^{*} \mathrm{y} 2$ $1618044355337867282217600^{*} \mathrm{u}[4]^{\wedge} 6^{*} \mathrm{x} 2^{\wedge} 2^{*} \mathrm{y} 2-5401844184114386012160^{*} \mathrm{u}[4]^{\wedge} 8^{*} \mathrm{x} 2 \wedge 2^{*} \mathrm{y} 2+$ $521939632737444480000^{*} \mathrm{u}[4]^{\wedge} 4^{*} \mathrm{x} 22^{\wedge} 2^{*} \mathrm{y} 2+162326375166524172701760^{*} \mathrm{u}[4]^{\wedge} 2^{*} \mathrm{x} 2^{\wedge} 2^{*} \mathrm{y} 2$ $86565031767376472363040 * \mathrm{x} 1{ }^{\wedge} 2^{*} \mathrm{u}[4]^{\wedge} 7^{*} \mathrm{y} 1+43350278294461384265760 * \mathrm{x} 1{ }^{\wedge} 2^{*} \mathrm{u}[4]^{\wedge} 3^{*} \mathrm{y} 1-$ $10013221280882645688960^{*} \mathrm{u}[4]^{*} \mathrm{x} 2^{\wedge} 2^{*} \mathrm{y} 1-260969816368722240000^{*} \mathrm{u}[4]^{\wedge} 2^{*} \mathrm{y} 1^{\wedge} 2^{*} \mathrm{x} 2+$ $130484908184361120000^{*} \mathrm{u}[4]^{\wedge} 8^{*} \mathrm{y} 1^{\wedge} 2^{*} \mathrm{x} 2-38053671310087840841436^{*} \mathrm{u}[4]^{\wedge} 2^{*} \mathrm{x} 1^{\wedge} 2^{*} \mathrm{x} 3+$ $17506332520566013632832^{*} \mathrm{u}[4]^{\wedge} 8^{*} \mathrm{x} 22^{\wedge} 2^{*} \mathrm{x} 3+2020240807451666342861^{*} \mathrm{u}[4]^{\wedge} 8^{*} \mathrm{x} 1{ }^{*} \mathrm{x} 2{ }^{*} \mathrm{x} 0$ $+38608562496854371769978^{*} \mathrm{u}[4]^{\wedge} 7^{*} \mathrm{x} 1^{*} \mathrm{x} 22^{*} \mathrm{x} 3+$ $215422510011315816355200^{*} \mathrm{u}[4]^{\wedge} 4^{*} \mathrm{x} 1^{*} \mathrm{x} 2 * \mathrm{x} 0+$ $37893831512473204003134^{*} \mathrm{u}[4]^{\wedge} 5^{*} \mathrm{x} 1^{*} \mathrm{x} 2^{*} \mathrm{x} 3-45137014077122235794466^{*} \mathrm{u}[4]^{\wedge} 3^{*} \mathrm{x} 1^{*} \mathrm{x} 2{ }^{*} \mathrm{x} 3$ - 64166610625591042731622*x1*x2*u[4]*x3+ $119476002196606019418322^{*} \mathrm{u}[4]^{\wedge} 6^{*} \mathrm{x} 1^{*} \times 2^{*} \mathrm{x} 0+$ $76202180281859822232878 * \mathrm{u}[4]^{\wedge} 2{ }^{*} \mathrm{x} 1{ }^{*} \mathrm{x} 2{ }^{*} \mathrm{x} 0-55527505353414469025280 * \mathrm{x} 2{ }^{*} \mathrm{y} 2{ }^{*} \mathrm{x} 3-$ $5815771820536792780800 * \mathrm{x} 22^{*} \mathrm{y} 3 * \mathrm{x} 3+46629840246011899603560 * \mathrm{y} 0 * \mathrm{x} 0 * \mathrm{x} 3-$ $14894046634003371061200^{*} \mathrm{y} 0 * \mathrm{x} 1 * \mathrm{x} 3-1479691023253837804800^{*} \mathrm{y} 1 * \mathrm{u}[4]^{\wedge} 5^{*} \mathrm{y} 0 * \mathrm{x} 1-$ $3235493335334612601600 * \mathrm{y} 1 * \mathrm{u}[4]^{*} \mathrm{y} 0{ }^{*} \mathrm{x} 1-1957821053833358438400^{*} \mathrm{u}[4]^{\wedge} 7^{*} \mathrm{y} 2{ }^{*} \mathrm{y} 3{ }^{*} \mathrm{x} 1+$ $2353325821249924684800^{*} \mathrm{u}[4]^{\wedge} 5^{*} \mathrm{y} 2^{*} \mathrm{y} 3^{*} \mathrm{x} 1+2353325821249924684800^{*} \mathrm{u}[4]^{\wedge} 3^{*} \mathrm{y} 2^{*} \mathrm{y} 3^{*} \mathrm{x} 1-$ $\left.1957821053833358438400^{*} \mathrm{u}[4]^{*} \mathrm{y} 2{ }^{*} \mathrm{y} 3^{*} \mathrm{x} 1+99702075931268366597760 * \mathrm{u}[4]\right]^{\wedge} 2^{*} \mathrm{x} 0{ }^{*} \mathrm{y} 2{ }^{*} \mathrm{x} 1+$ $3303266503925151878400^{*} \mathrm{u}[4]^{\wedge} 6^{*} \mathrm{y} 2^{*} \mathrm{y} 0 * \mathrm{x} 1-127148794172396092290960^{*} \mathrm{u}[4]^{*} \mathrm{x} 0{ }^{*} \mathrm{y} 1^{*} \mathrm{x} 1-$ $49438095927070780800^{*} \mathrm{y} 1 * \mathrm{u}[4]^{\wedge} 8^{*} \mathrm{y} 0 * \mathrm{x} 2+4014518299520858438400^{*} \mathrm{y} 1{ }^{*} \mathrm{u}[4]^{\wedge} 6^{*} \mathrm{y} 0 * \mathrm{x} 2-$ $4014518299520858438400^{*} \mathrm{y} 1^{*} \mathrm{u}[4]^{\wedge} 2^{*} \mathrm{y} 0{ }^{*} \mathrm{x} 2-18174492306614674346469^{*} \mathrm{x} 1 \wedge 2^{*} \mathrm{u}[4]^{\wedge} 8^{*} \mathrm{x} 2+$ $56615622029053807584084^{*} \mathrm{x} 1^{*} \mathrm{u}[4]^{\wedge} 7^{*} \mathrm{x} 22^{\wedge} 2+6603540180878004880902^{*} \mathrm{x} 1^{\wedge} 3^{*} \mathrm{u}[4]-$ $3591595853839046327040 * \mathrm{x} 00^{\wedge} 2^{*} \mathrm{y} 0+589398044823556300800^{*} \mathrm{y} 22^{*}{ }^{*} \mathrm{x} 3+$ $42952524794107353573840 * \mathrm{y} 2^{*} \mathrm{x} 1 \wedge 2+3194124073124161396320^{*} \mathrm{u}[4]^{\wedge} 8^{*} \mathrm{y} 3^{*} \mathrm{x} 1{ }^{*} \mathrm{x} 2-$ $108468030624141292637760 * \mathrm{u}[4]^{\wedge} 6^{*} \mathrm{y} 3^{*} \mathrm{x} 1{ }^{*} \mathrm{x} 2+$ $63995279268719563315200^{*} \mathrm{u}[4]^{\wedge} 4^{*} \mathrm{y} 3^{*} \mathrm{x} 1{ }^{*} \mathrm{x} 2+2033452921899278131200^{*} \mathrm{y} 0 * \mathrm{y} 1{ }^{*} \mathrm{u}[4]^{\wedge} 7^{*} \mathrm{x} 2$ $+74889370180197834393600 * \mathrm{y} 0 * \mathrm{y} 1{ }^{*} \mathrm{u}[4]^{\wedge} 5 * \mathrm{x} 2+$ $74889370180197834393600^{*} \mathrm{y} 0^{*} \mathrm{y} 1^{*} \mathrm{u}[4]^{\wedge} 3^{*} \mathrm{x} 2+2033452921899278131200^{*} \mathrm{y} 0^{*} \mathrm{y} 1{ }^{*} \mathrm{u}[4]^{*} \mathrm{x} 2-$ $16833839702634059788800^{*} \mathrm{y} 0^{*} \mathrm{u}[4]^{\wedge} 7^{*} \mathrm{y} 2^{*} \mathrm{x} 1+$ $18287492306597820633600^{*} \mathrm{y} 0^{*} \mathrm{u}[4]^{\wedge} 3^{*} \mathrm{y} 2^{*} \mathrm{x} 1+7843268600165418531840^{*} \mathrm{y} 0 * \mathrm{u}[4]^{\wedge} 8^{*} \mathrm{x} 1^{\wedge} 2-$ $40179755997427921269120^{*} \mathrm{y} 0 * \mathrm{u}[4]^{\wedge} 6^{*} \mathrm{x} 1^{\wedge} 2+35121332009231880422400^{*} \mathrm{y} 0 * \mathrm{u}[4]^{\wedge} 4^{*} \mathrm{x} 1^{\wedge} 2$ $+6512076592159801691520^{*} \mathrm{y} 0 * \mathrm{u}[4]^{\wedge} 2^{*} \mathrm{x} 1^{\wedge} 2-70488777813526425600^{*} \mathrm{y} 1^{\wedge} 2^{*} \mathrm{x} 0-$ $130484908184361120000^{*} \mathrm{y} 1^{\wedge} 2^{*} \mathrm{x} 2+1163244578015311459200^{*} \mathrm{y} 1^{\wedge} 2^{*} \mathrm{x} 3-$ $99578427514393651200 * \mathrm{y} 0^{\wedge} 2 * \mathrm{x} 1+190565470287648518400^{*} \mathrm{y}^{\wedge} 2^{*} \mathrm{x} 2-$ $1752642622838867760000^{*} \mathrm{y} 0{ }^{\wedge} 2^{*} \mathrm{x} 3-36178817214587586484320^{*} \mathrm{x} 3{ }^{*} \mathrm{y} 2 * \mathrm{x} 0-$ $36650394224513165491200^{*} \mathrm{y} 2^{\wedge} 2^{*} \mathrm{x} 0^{*} \mathrm{u}[4]^{\wedge} 6+36650394224513165491200^{*} \mathrm{y} 2^{\wedge} 2^{*} \mathrm{x} 00^{*} \mathrm{u}[4]^{\wedge} 2-$ $1127944258631582745600 * \mathrm{y} 2^{\wedge} 2^{*} \mathrm{x} 0 * \mathrm{u}[4]^{\wedge} 8+17338230409252052851200^{*} \mathrm{y} 2^{\wedge} 2^{*} \mathrm{u}[4]^{\wedge} 7^{*} \mathrm{x} 3-$ $16774320186743841446400^{*} \mathrm{y} 2^{\wedge} 2^{*} \mathrm{u}[4]^{\wedge} 5^{*} \mathrm{x} 3-16774320186743841446400^{*} \mathrm{y} 2^{\wedge} 2^{*} \mathrm{u}[4]^{\wedge} 3^{*} \mathrm{x} 3+$ $17338230409252052851200^{*} \mathrm{y} 2^{\wedge} 2^{*} \mathrm{u}[4]^{*} \mathrm{x} 3+70488777813526425600 * \mathrm{u}[4]^{\wedge} 8^{*} \mathrm{y} 1^{\wedge} 2^{*} \mathrm{x} 0-$ $19453141370888165491200^{*} \mathrm{u}[4]^{\wedge} 7^{*} \mathrm{y} 1^{\wedge} 2^{*} \mathrm{x} 3+10429587301835503526400^{*} \mathrm{u}[4]^{\wedge} 5^{*} \mathrm{y} 11^{\wedge} 2^{*} \mathrm{x} 3$ $+10429587301835503526400^{*} \mathrm{u}[4]^{\wedge} 3^{*} \mathrm{y} 1^{\wedge} 2^{*} \mathrm{x} 3-$ 
$1193359969436648926629144^{*} \mathrm{u}[4]^{\wedge} 5^{*} \mathrm{x} 3^{*} \mathrm{x} 2 \wedge 2-$

$941017965305159994755544^{*} \mathrm{u}[4]^{\wedge} 3^{*} \mathrm{x} 3^{*} \mathrm{x} 2^{\wedge} 2-122072793942331948072224^{*} \mathrm{x} 00^{*} \mathrm{u}[4]^{\wedge} 8^{*} \mathrm{x} 1^{\wedge} 2$ - $304844983723721088995952^{*} \mathrm{x} 0^{*} \mathrm{u}[4]^{\wedge} 2^{*} \mathrm{x} 1^{\wedge} 2+$ $678675471597543593812752 * \mathrm{x} 0 * \mathrm{u}[4]^{\wedge} 2^{*} \mathrm{x} 22^{\wedge} 2+$ $266647622541808383918744^{*} \mathrm{u}[4]^{\wedge} 5^{*} \mathrm{x} 3^{*} \mathrm{x} 1^{\wedge} 2+88020824383736350629144^{*} \mathrm{u}[4]^{\wedge} 3^{*} \mathrm{x} 3^{*} \mathrm{x} 1^{\wedge} 2$ - $9615352887762139065600^{*} \mathrm{y} 3^{*} \mathrm{y} 2 * \mathrm{x} 1+3705176690528787302400^{*} \mathrm{y} 0^{\wedge} 2^{*} \mathrm{x} 1^{*} \mathrm{u}[4]^{\wedge} 6-$ $17506332520566013632832{ }^{*} \times 2^{\wedge} 2^{*} \mathrm{x} 3+27050816406346276574676^{*} \times 0^{\wedge} 2^{*} \mathrm{x} 2$ $144344370709761744313767^{*} \mathrm{x} 00^{\wedge} 2^{*} \mathrm{x} 3+122072793942331948072224^{*} \mathrm{x} 1^{\wedge} 2^{*} \mathrm{x} 0+$ $11835145962415413443880 * \mathrm{x} 1 \wedge 2^{*} \mathrm{y} 3+359054157602047749120^{*} \mathrm{x} 22^{*} \mathrm{y} 3+$ $223631302071428595011232^{*} \mathrm{x} 0 \wedge 2^{*} \mathrm{x} 1+1762072189180358889600^{*} \mathrm{y} 3^{\wedge} 2^{*} \mathrm{u}[4]^{\wedge} 5^{*} \mathrm{x} 1+$ $5931091146019074796800^{*} \mathrm{y}^{\wedge} 2^{*} \mathrm{u}[4]^{\wedge} 6^{*} \mathrm{x} 2+6192060962387797036800^{*} \mathrm{u}[4]^{\wedge} 2^{*} \mathrm{y} 0 \wedge 2^{*} \mathrm{x} 2+$ $60080562103287398400^{*} \mathrm{y} 3^{\wedge} 2^{*} \mathrm{u}[4]^{\wedge} 8^{*} \mathrm{x} 2-3286595951481547036800^{*} \mathrm{y} 3^{\wedge} 2^{*} \mathrm{u}[4]^{\wedge} 7^{*} \mathrm{x} 1+$ $1762072189180358889600^{*} \mathrm{y} 3^{\wedge} 2^{*} \mathrm{u}[4]^{\wedge} 3^{*} \mathrm{x} 1-12525421186940126750400^{*} \mathrm{x} 2{ }^{*} \mathrm{u}[4]^{\wedge} 8^{*} \mathrm{y} 0{ }^{*} \mathrm{x} 3-$ $18222462145283853177600^{*} \mathrm{x} 2 * \mathrm{u}[4]^{\wedge} 6^{*} \mathrm{y} 0 * \mathrm{x} 3+$

$29597307581988273254400 *^{*} 2{ }^{*} \mathrm{u}[4]^{\wedge} 4^{*} \mathrm{y} 0 *^{*} \mathrm{x} 3-18764583703732674662400{ }^{*} \mathrm{y} 0{ }^{*} \mathrm{y} 1{ }^{*} \mathrm{u}[4]^{\wedge} 7^{*} \mathrm{x} 3$ $-63305790782198023987200^{*} \mathrm{y} 0 * \mathrm{y} 1 * \mathrm{u}[4]^{\wedge} 5 * \mathrm{x} 3-$

$63305790782198023987200^{*} \mathrm{y} 0{ }^{*} \mathrm{y} 1{ }^{*} \mathrm{u}[4]^{\wedge} 3^{*} \mathrm{x} 3-18764583703732674662400^{*} \mathrm{y} 0{ }^{*} \mathrm{y} 1^{*} \mathrm{u}[4]^{*} \mathrm{x} 3+$ $229955038619269017600^{*} \mathrm{y} 0 *^{*} \mathrm{u}[4]^{\wedge} 7^{*} \mathrm{y} 2^{*} \mathrm{x} 0+7701904786857807052800^{*} \mathrm{y} 0 * \mathrm{u}[4]^{\wedge} 3^{*} \mathrm{y} 2{ }^{*} \mathrm{x} 0-$ $8204524479548535686400^{*} \mathrm{x} 0 * \mathrm{y} 0 * \mathrm{x} 1+7866808208600772168960^{*} \mathrm{y} 1^{*} \mathrm{x} 0{ }^{*} \mathrm{x} 1-$ $589398044823556300800^{*} \mathrm{y} 3{ }^{*} \mathrm{y} 2{ }^{*} \mathrm{x} 2+60080562103287398400 * \mathrm{y} 3{ }^{*} \mathrm{y} 2{ }^{*} \mathrm{x} 3-$ $454502089651994841600^{*} \mathrm{y} 1{ }^{*} \mathrm{y} 2{ }^{*} \mathrm{x} 0+130484908184361120000^{*} \mathrm{y} 1{ }^{*} \mathrm{y} 2 * \mathrm{x} 1-$ $181706575495470105600^{*} \mathrm{y} 1{ }^{*} \mathrm{y} 3^{*} \mathrm{x} 2+25618126687843314952080^{*} \mathrm{x} 00^{\wedge} 2^{*} \mathrm{u}[4]^{\wedge} 3^{*} \mathrm{y} 0+$ $29123411933521050472080^{*} \mathrm{x} 0^{\wedge} 2^{*} \mathrm{u}[4]^{\wedge} 5^{*} \mathrm{y} 0+95293032757795736233920^{*} \mathrm{x} 0^{\wedge} 2^{*} \mathrm{u}[4]^{\wedge} 6^{*} \mathrm{y} 3-$ $85568190195152210377680^{*} \mathrm{x} 3^{\wedge} 2^{*} \mathrm{u}[4]^{*} \mathrm{y} 0-224815770826237998748080^{*} \mathrm{x} 3^{\wedge} 2^{*} \mathrm{u}[4]^{\wedge} 3^{*} \mathrm{y} 0+$ $7010570491355471040000^{*} \mathrm{x} 3^{\wedge} 2^{*} \mathrm{y} 3^{*} \mathrm{u}[4]^{\wedge} 4-217805200334882527708080^{*} \mathrm{u}[4]^{\wedge} 5^{*} \mathrm{x} 33^{\wedge} 2^{*} \mathrm{y} 0$ $20130604930803169501920^{*} \mathrm{u}[4]^{\wedge} 6^{*} \mathrm{x} 3^{\wedge} 2^{*} \mathrm{y} 3-78557619703796739337680^{*} \mathrm{u}[4]^{\wedge} 7^{*} \mathrm{x} 3^{\wedge} 2^{*} \mathrm{y} 0-$ $5598387771274662174720^{*} \mathrm{u}[4]^{\wedge} 8^{*} \mathrm{x} 3^{\wedge} 2^{*} \mathrm{y} 3-81271891775084794153920^{*} \mathrm{x} 0^{\wedge} 2^{*} \mathrm{u}[4]^{\wedge} 2^{*} \mathrm{y} 3+$ $27141175422158640541920^{*} \mathrm{u}[4]^{\wedge} 2^{*} \mathrm{x} 3^{\wedge} 2^{*} \mathrm{y} 3+19168975482353982445680^{*} \mathrm{x} 0^{\wedge} 2^{*} \mathrm{u}[4]^{\wedge} 7^{*} \mathrm{y} 0$ $+37921673816254342260720^{*} \mathrm{x} 0^{\wedge} 2^{*} \mathrm{u}[4]^{\wedge} 8^{*} \mathrm{y} 3-324361164700473675721752^{*} \mathrm{u}[4]^{*} \mathrm{x} 3^{*} \mathrm{x} 1^{\wedge} 2$ $5235790503263664314880^{*} \mathrm{y} 1^{*} \mathrm{u}[4]^{\wedge} 8^{*} \mathrm{x} 1^{\wedge} 2-42111052042871394328320^{*} \mathrm{y} 1^{*} \mathrm{u}[4]^{\wedge} 6^{*} \mathrm{x} 2^{\wedge} 2-$ $291378868314391437120^{*} \mathrm{y} 1^{*} \mathrm{u}[4]^{\wedge} 8^{*} \mathrm{x} 22^{\wedge} 2-4364243622796112640000^{*} \mathrm{y} 1^{*} \mathrm{u}[4]^{\wedge} 4^{*} \mathrm{x} 1^{\wedge} 2+$ $33382564797279169048320^{*} \mathrm{y} 1^{*} \mathrm{u}[4]^{\wedge} 2^{*} \mathrm{x} 22^{\wedge} 2+12652594242545945316864^{*} \mathrm{x} 3^{\wedge} 3+$ $1824208608129867639552^{*} \times 2^{\wedge} 3-31611853833646616347632^{*} \times 3^{\wedge} 2^{*} \mathrm{x} 1+$ $22739296111328492404176^{*} \mathrm{x} 00^{*} \mathrm{x} 2^{\wedge} 2+64876165978482902342160^{*} \mathrm{x} 0 * \mathrm{u}[4]^{\wedge} 2^{*} \mathrm{x} 3{ }^{*} \mathrm{y} 0-$ $7010570491355471040000 * \mathrm{x} 0 * \mathrm{u}[4]^{\wedge} 4 * \mathrm{x} 3 * \mathrm{y} 0+$ $290465513487164110533840^{*} \mathrm{x} 0 * \mathrm{u}[4]^{\wedge} 5^{*} \mathrm{x} 3^{*} \mathrm{y} 3-$ $71886736469838373382160^{*} \mathrm{x} 0 * \mathrm{u}[4]^{\wedge} 6^{*} \mathrm{x} 3^{*} \mathrm{y} 0+$ $79067923235253350774640^{*} \mathrm{x} 0^{*} \mathrm{u}[4]^{\wedge} 7^{*} \mathrm{x} 3^{*} \mathrm{y} 3+82573208480931086294640^{*} \mathrm{x} 00^{*} \mathrm{u}[4]^{*} \mathrm{x} 3^{*} \mathrm{y} 3$ $+293970798732841846053840^{*} \mathrm{x} 0 * \mathrm{u}[4]^{\wedge} 3^{*} \mathrm{x} 33^{*} \mathrm{y} 3-$

$46629840246011899603560 * \mathrm{x} 0 * \mathrm{u}[4]^{\wedge} 8^{*} \mathrm{x} 33^{*} \mathrm{y} 0+14021140982710942080000^{*} \mathrm{x} 0{ }^{\wedge} 2^{*} \mathrm{u}[4]^{\wedge} 4^{*} \mathrm{y} 3$ $+19583611217015889133440^{*} \mathrm{x} 1^{\wedge} 2^{*} \mathrm{u}[4]^{\wedge} 7^{*} \mathrm{y} 0-13407911452274355262080^{*} \mathrm{x} 11^{\wedge} 2^{*} \mathrm{u}[4]^{\wedge} 5^{*} \mathrm{y} 0$ - 20824572447574507140480*x1^2* $2^{*}[4]^{\wedge} 3^{*} \mathrm{y} 0-11835145962415413443880^{*} \mathrm{x} 1^{\wedge} 2^{*} \mathrm{u}[4]^{\wedge} 8^{*} \mathrm{y} 3$ $+4311146875083283123200^{*} \mathrm{x} 1^{\wedge} 2^{*} \mathrm{u}[4]^{\wedge} 4^{*} \mathrm{y} 3-16828662920075966942640^{*} \mathrm{x} 1^{\wedge} 2^{*} \mathrm{u}[4]^{\wedge} 2^{*} \mathrm{y} 3$ $+12913020812409250065840^{*} \mathrm{x} 1^{\wedge} 2^{*} \mathrm{y} 3^{*} \mathrm{u}[4]^{\wedge} 6-359054157602047749120^{*} \mathrm{u}[4]^{\wedge} 8^{*} \mathrm{x} 2^{\wedge} 2^{*} \mathrm{y} 3-$ $38012672848039879011840^{*} \mathrm{u}[4]^{\wedge} 6^{*} \mathrm{x} 2^{\wedge} 2^{*} \mathrm{y} 3-5733650466274696243200^{*} \mathrm{u}[4]^{\wedge} 4^{*} \mathrm{x} 22^{\wedge} 2^{*} \mathrm{y} 3+$ 
$40505811364515182768640^{*} \mathrm{u}[4]^{\wedge} 2^{*} \mathrm{x} 2^{\wedge} 2^{*} \mathrm{y} 3-5292190581022112601600^{*} \mathrm{u}[4]^{\wedge} 2^{*} \mathrm{y} 2^{*} \mathrm{y} 3^{*} \mathrm{x} 2+$ $71594587579861244060952^{*} \mathrm{u}[4]^{*} \mathrm{x} 3^{*} \mathrm{x} 2^{\wedge} 2+11160404949161409860160^{*} \mathrm{u}[4]^{\wedge} 7^{*} \mathrm{x} 2^{*} \mathrm{y} 2{ }^{*} \mathrm{x} 0+$ $55527505353414469025280^{*} \mathrm{u}[4]^{\wedge} 8^{*} \mathrm{x} 2^{*} \mathrm{y} 2^{*} \mathrm{x} 3-27527694207911551566720^{*} \mathrm{u}[4]^{\wedge} 5^{*} \mathrm{x} 2{ }^{*} \mathrm{y} 2^{*} \mathrm{x} 0$ $-33666682921710449281920 * \mathrm{u}[4]^{\wedge} 3^{*} \mathrm{x} 2 * \mathrm{y} 2 * \mathrm{x} 0-$ $128566279149122725526160^{*} \mathrm{x} 1^{*} \mathrm{u}[4]^{\wedge} 7^{*} \mathrm{y} 1^{*} \mathrm{x} 0+$ $2299110435146754927720^{*} \mathrm{x} 1^{*} \mathrm{u}[4]^{\wedge} 8^{*} \mathrm{y} 1^{*} \mathrm{x} 3-311540369186818026676080^{*} \mathrm{x} 1{ }^{*} \mathrm{u}[4]^{\wedge} 3^{*} \mathrm{y} 1^{*} \mathrm{x} 0$ $+35931041669573173376640^{*} \mathrm{u}[4]^{\wedge} 6^{*} \mathrm{x} 2^{*} \mathrm{y} 1^{*} \mathrm{x} 0-$ $46379876494436320026240^{*} \mathrm{u}[4]^{\wedge} 2^{*} \mathrm{x} 2{ }^{*} \mathrm{y} 1{ }^{*} \mathrm{x} 0-86304061951007750123040^{*} \mathrm{x} 1{ }^{\wedge} 2^{*} \mathrm{y} 1{ }^{*} \mathrm{u}[4]+$ $128184642384814776093120^{*} \mathrm{x} 2 * \mathrm{u}[4]^{\wedge} 5^{*} \mathrm{y} 0 * \mathrm{x} 1+$ $150601734802764913222080^{*} \mathrm{x} 1{ }^{*} \mathrm{u}[4]^{*} \mathrm{x} 22^{*} \mathrm{y} 2+260969816368722240000^{*} \mathrm{u}[4]^{*} \mathrm{y} 2^{\wedge} 2^{*} \mathrm{x} 1+$ $21711208940383930674468^{*} \mathrm{x} 1^{\wedge} 2^{*} \mathrm{u}[4]^{\wedge} 8^{*} \mathrm{x} 3-156946908337589031294042^{*} \mathrm{u}[4]^{\wedge} 5^{*} \mathrm{x} 1^{\wedge} 2^{*} \mathrm{x} 0$ $+29325155475420915543036^{*} \mathrm{u}[4]^{\wedge} 6^{*} \mathrm{x} 1^{\wedge} 2^{*} \mathrm{x} 3-18600679601091912650400^{*} \mathrm{u}[4]^{\wedge} 4^{*} \mathrm{x} 1^{\wedge} 2^{*} \mathrm{x} 3$ $+29561909517320823322560 * \mathrm{y} 1 * \mathrm{u}[4]^{\wedge} 7^{*} \mathrm{x} 1{ }^{*} \mathrm{x} 2-$

$64334717804619637928640^{*} \mathrm{y} 1^{*} \mathrm{u}[4]^{\wedge} 3^{*} \mathrm{x} 1^{*} \mathrm{x} 2-62152595993221581608640^{*} \mathrm{y} 1{ }^{*} \mathrm{u}[4]^{\wedge} 5^{*} \mathrm{x} 1{ }^{*} \mathrm{x} 2$ $+4346601035912351825760 * \mathrm{u}[4]^{\wedge} 8 * \mathrm{y} 2 * \mathrm{x} 1 * \mathrm{x} 2-$

$63452777483670237656640^{*} \mathrm{u}[4]^{\wedge} 6^{*} \mathrm{y} 2^{*} \mathrm{x} 1^{*} \mathrm{x} 2+47833590034801437085440^{*} \mathrm{x} 0{ }^{\wedge} 2^{*} \mathrm{u}[4]^{\wedge} 6^{*} \mathrm{y} 0$ $-20536055123737203661440^{*} \mathrm{u}[4]^{\wedge} 6^{*} \mathrm{x} 3^{\wedge} 2^{*} \mathrm{y} 0+6884805159238901424960^{*} \mathrm{u}[4]^{\wedge} 8^{*} \mathrm{x} 3^{\wedge} 2^{*} \mathrm{y} 0$ $+8459643846544450560000^{*} \mathrm{u}[4]^{\wedge} 4^{*} \mathrm{x} 3^{\wedge} 2^{*} \mathrm{y} 0+28995698970281654221440^{*} \mathrm{u}[4]^{\wedge} 2^{*} \mathrm{x} 3^{\wedge} 2^{*} \mathrm{y} 0$ $-7613044325901925685760^{*} \mathrm{x} 0^{\wedge} 2^{*} \mathrm{u}[4]^{\wedge} 7^{*} \mathrm{y} 3+62691485599813137085440^{*} \mathrm{x} 0^{\wedge} 2^{*} \mathrm{u}[4]^{\wedge} 3^{*} \mathrm{y} 3-$ $43603768111529211805440^{*} \mathrm{x} 0^{\wedge} 2^{*} \mathrm{u}[4]^{\wedge} 2^{*} \mathrm{y} 0+58461663676540911805440^{*} \mathrm{x} 0 \wedge 2^{*} \mathrm{y} 3^{*} \mathrm{u}[4]^{\wedge} 5$ $+1224375959472605746560^{*} \mathrm{u}[4]^{\wedge} 5^{*} \mathrm{x} 3^{\wedge} 2^{*} \mathrm{y} 3+25393479909603652229760^{*} \mathrm{u}[4]^{\wedge} 7^{*} \mathrm{x} 3^{\wedge} 2^{*} \mathrm{y} 3$ $+27508390871239764869760^{*} \mathrm{u}[4]^{*} \mathrm{x} 3^{\wedge} 2^{*} \mathrm{y} 3+3339286921108718386560^{*} \mathrm{u}[4]^{\wedge} 3^{*} \mathrm{x} 3^{\wedge} 2^{*} \mathrm{y} 3+$ $75522007100500892570880^{*} \mathrm{u}[4]^{\wedge} 3^{*} \mathrm{x} 3^{*} \mathrm{y} 0{ }^{*} \mathrm{x} 0-22739296111328492404176^{*} \mathrm{x} 0{ }^{*} \mathrm{u}[4]^{\wedge} 8^{*} \mathrm{x} 22^{\wedge} 2$ $+13248205218112512144960^{*} \mathrm{u}[4]^{*} \mathrm{y} 2^{*} \mathrm{x} 0^{*} \mathrm{x} 2-1824208608129867639552^{*} \mathrm{u}[4]^{\wedge} 8^{*} \mathrm{x} 2^{\wedge} 3+$ $77181861893368692675054^{*} \mathrm{x} 1^{\wedge} 2^{*} \mathrm{x} 2^{*} \mathrm{u}[4]^{\wedge} 2-10515855737033206560000^{*} \mathrm{u}[4]^{\wedge} 5^{*} \mathrm{y} 3^{\wedge} 2^{*} \mathrm{x} 0-$ $3505285245677735520000^{*} \mathrm{u}[4]^{\wedge} 7^{*} \mathrm{y} 3^{\wedge} 2^{*} \mathrm{x} 0-28874591488317226504320^{*} \mathrm{y} 1^{*} \mathrm{x} 1^{\wedge} 2^{*} \mathrm{u}[4]^{\wedge} 2+$ $24510347865521113864320^{*} \mathrm{y} 1^{*} \mathrm{u}[4]^{\wedge} 6^{*} \mathrm{x} 1^{\wedge} 2+77000894035025805621120^{*} \mathrm{u}[4]^{\wedge} 7^{*} \mathrm{y} 2{ }^{*} \mathrm{x} 0{ }^{*} \mathrm{x} 1$ $-42226305546474056255040^{*} \mathrm{u}[4]^{\wedge} 8^{*} \mathrm{y} 2^{*} \mathrm{x} 3^{*} \mathrm{x} 1+$

$510948243186559577410296^{*} \mathrm{x} 0 * \mathrm{u}[4]^{\wedge} 7^{*} \mathrm{x} 2{ }^{*} \mathrm{x} 1-7866808208600772168960^{*} \mathrm{y} 1{ }^{*} \mathrm{u}[4]^{\wedge} 8^{*} \mathrm{x} 00^{*} \mathrm{x} 1$ $+22895211083760172042560^{*} \mathrm{u}[4]^{\wedge} 3^{*} \mathrm{y} 1^{*} \mathrm{x} 3^{*} \mathrm{x} 1-$ $699958841184938501191512^{*} \mathrm{x} 0 * \mathrm{u}[4]^{\wedge} 3^{*} \mathrm{x} 2^{*} \mathrm{x} 1+$ $1275124476258575595081096^{*} \mathrm{u}[4]^{\wedge} 6^{*} \mathrm{x} 3^{*} \mathrm{x} 2^{*} \mathrm{x} 1$ $243455424452398658486400^{*} \mathrm{u}[4]^{\wedge} 4^{*} \mathrm{x} 3^{*} \mathrm{x} 22^{*} \mathrm{x} 1$ $1023794156609595636095496^{*} \mathrm{u}[4]^{\wedge} 2^{*} \mathrm{x} 3^{*} \mathrm{x} 2{ }^{*} \mathrm{x} 1+$ $512916966985704902535096^{*} \mathrm{x} 0 * \mathrm{u}[4]^{*} \mathrm{x} 2{ }^{*} \mathrm{x} 1-78094520594806767667200^{*} \mathrm{y} 1{ }^{*} \mathrm{u}[4]^{\wedge} 4^{*} \mathrm{x} 0{ }^{*} \mathrm{x} 1$ $+96118899543391286315520^{*} \mathrm{u}[4]^{\wedge} 6^{*} \mathrm{y} 2^{*} \mathrm{x} 3^{*} \mathrm{x} 1$ $33103769182759908172800^{*} \mathrm{u}[4]^{\wedge} 4^{*} \mathrm{y} 2^{*} \mathrm{x} 3^{*} \mathrm{x} 1-60433657311651194488320^{*} \mathrm{u}[4]^{\wedge} 2^{*} \mathrm{y} 2^{*} \mathrm{x} 3^{*} \mathrm{x} 1$ - $82810988589262087529280^{*} \mathrm{y} 1^{*} \mathrm{u}[4]^{*} \mathrm{x} 3^{*} \mathrm{x} 1+100743376845076414734720^{*} \mathrm{u}[4]^{*} \mathrm{y} 2^{*} \mathrm{x} 00^{*} \mathrm{x} 1-$ $3536818019090481715200^{*} \mathrm{u}[4]^{\wedge} 7^{*} \mathrm{y} 0{ }^{*} \mathrm{y} 3^{*} \mathrm{x} 1+15399091795240857600^{*} \mathrm{u}[4]^{\wedge} 8^{*} \mathrm{y} 0{ }^{*} \mathrm{y} 3^{*} \mathrm{x} 2+$ $7042837854590481715200^{*} \mathrm{u}[4]^{\wedge} 6^{*} \mathrm{y} 0^{*} \mathrm{y} 3^{*} \mathrm{x} 2+4449163125221179972560^{*} \mathrm{u}[4]^{\wedge} 7^{*} \mathrm{x} 22^{\wedge} 2^{*} \mathrm{y} 0+$ $86217486274581269140080^{*} \mathrm{u}[4]^{\wedge} 5^{*} \mathrm{x} 22^{\wedge} 2^{*} \mathrm{y} 0+94345399065477127578480^{*} \mathrm{u}[4]^{\wedge} 3^{*} \mathrm{x} 2^{\wedge} 2^{*} \mathrm{y} 0$ $+15663690236676246925680^{*} \mathrm{x} 0^{\wedge} 2^{*} \mathrm{u}[4]^{*} \mathrm{y} 0-1300727151043926278400^{*} \mathrm{y} 1^{*} \mathrm{y} 3^{*} \mathrm{x} 0-$ $1163244578015311459200^{*} \mathrm{y} 1{ }^{*} \mathrm{y} 3{ }^{*} \mathrm{x} 1+99578427514393651200^{*} \mathrm{y} 0 * \mathrm{y} 1{ }^{*} \mathrm{x} 0+$ $70488777813526425600^{*} \mathrm{y} 0 *^{*} 1^{*} \mathrm{x} 1+49438095927070780800^{*} \mathrm{y} 00^{*} \mathrm{y} 1{ }^{*} \mathrm{x} 2-$ $402651386800134638400^{*} \mathrm{y} 0{ }^{*} \mathrm{y} 1{ }^{*} \mathrm{x} 3-65404861482304852019280^{*} \mathrm{u}[4]^{\wedge} 8^{*} \mathrm{x} 1^{*} \mathrm{y} 2^{*} \mathrm{x} 0-$ 
$2222045842113146649600^{*} \mathrm{x} 2 * \mathrm{u}[4]^{\wedge} 4^{*} \mathrm{y} 1 * \mathrm{x} 0+$

$407395351416488135111280^{*} \mathrm{x} 2{ }^{*} \mathrm{u}[4]^{\wedge} 5^{*} \mathrm{y} 1{ }^{*} \mathrm{x} 3-7317784803446010316800^{*} \mathrm{u}[4]^{\wedge} 6^{*} \mathrm{y} 1{ }^{*} \mathrm{y} 2{ }^{*} \mathrm{x} 0$

$+3204390312071010316800^{*} \mathrm{u}[4]^{\wedge} 7^{*} \mathrm{y} 1{ }^{*} \mathrm{y} 2^{*} \mathrm{x} 3+454502089651994841600^{*} \mathrm{u}[4]^{\wedge} 8^{*} \mathrm{y} 1{ }^{*} \mathrm{y} 2{ }^{*} \mathrm{x} 0$

$+13221598731789208550400^{*} \mathrm{u}[4]^{\wedge} 4^{*} \mathrm{x} 1^{*} \mathrm{y} 2 * \mathrm{x} 0$ -

$11545950143247980760720^{*} \mathrm{u}[4]^{\wedge} 5^{*} \mathrm{x} 1^{*} \mathrm{y} 2^{*} \mathrm{x} 3+100257681200887700997120^{*} \mathrm{u}[4]^{*} \mathrm{y} 2^{*} \mathrm{x} 3^{\wedge} 2$

$+22513157218457053339200^{*} \mathrm{u}[4]^{*} \mathrm{y} 3^{*} \mathrm{x} 1{ }^{*} \mathrm{x} 0-4229821923272225280000^{*} \mathrm{x} 0{ }^{*} \mathrm{u}[4]^{\wedge} 4^{*} \mathrm{y} 3^{*} \mathrm{x} 3$

$+77636918062137005210880 * \mathrm{x} 0 * \mathrm{u}[4]^{\wedge} 5 * \mathrm{y} 0 * \mathrm{x} 3-$

$66504560620666670522880 * \mathrm{x} 0 * \mathrm{u}[4]^{\wedge} 6^{*} \mathrm{y} 3 * \mathrm{x} 3+$

$62274738697394445242880 * \mathrm{x} 0 * \mathrm{u}[4]^{\wedge} 2^{*} \mathrm{y} 3{ }^{*} \mathrm{x} 3-26911617774361382411520{ }^{*} \mathrm{x} 0 * \mathrm{u}[4]{ }^{*} \mathrm{y} 0 * \mathrm{x} 3-$

$3383222402629700405760^{*} \mathrm{x} 0^{\wedge} 2^{*} \mathrm{y} 3^{*} \mathrm{u}[4]+3591595853839046327040^{*} \mathrm{x} 0 \wedge{ }^{\wedge} 2^{*} \mathrm{u}[4]^{\wedge} 8^{*} \mathrm{y} 0+$

$4229821923272225280000^{*} \mathrm{x} 00^{\wedge} 2^{*} \mathrm{u}[4]^{\wedge} 4^{*} \mathrm{y} 0-17338230409252052851200^{*} \mathrm{y} 3^{*} \mathrm{y} 2^{*} \mathrm{u}[4]^{*} \mathrm{x} 2-$

$3286595951481547036800^{*} \mathrm{y} 3^{\wedge} 2^{*} \mathrm{u}[4]^{*} \mathrm{x} 1-5931091146019074796800^{*} \mathrm{y} 3^{\wedge} 2^{*} \mathrm{x} 2{ }^{*} \mathrm{u}[4]^{\wedge} 2$ -

$2544981638286525609600^{*} \mathrm{u}[4]^{\wedge} 3^{*} \mathrm{y} 0^{\wedge} 2^{*} \mathrm{x} 1-2544981638286525609600^{*} \mathrm{u}[4]^{\wedge} 5^{*} \mathrm{y} 0^{\wedge} 2^{*} \mathrm{x} 1-$

$6192060962387797036800^{*} \mathrm{u}[4]^{\wedge} 6^{*} \mathrm{y} 0^{\wedge} 2^{*} \mathrm{x} 2+3025626135112824796800^{*} \mathrm{u}[4]^{\wedge} 7^{*} \mathrm{y} 0^{\wedge} 2^{*} \mathrm{x} 1-$

$190565470287648518400^{*} \mathrm{u}[4]^{\wedge} 8^{*} \mathrm{y} 0^{\wedge} 2^{*} \mathrm{x} 2-6192060962387797036800^{*} \mathrm{u}[4]^{\wedge} 2^{*} \mathrm{y} 2^{*} \mathrm{y} 0{ }^{*} \mathrm{x} 0-$

$32821252489809599144760^{*} \mathrm{u}[4]^{\wedge} 8^{*} \mathrm{x} 0 * \mathrm{y} 1^{*} \mathrm{x} 3+$

$426132532507020389804880^{*} \mathrm{u}[4]^{\wedge} 6^{*} \mathrm{x} 0 * \mathrm{y} 1{ }^{*} \mathrm{x} 3+$

$10619275914061256332800^{*} \mathrm{u}[4]^{\wedge} 4^{*} \mathrm{x} 0^{*} \mathrm{y} 1^{*} \mathrm{x} 3+782909449106166720000^{*} \mathrm{y} 2^{\wedge} 2^{*} \mathrm{u}[4]^{\wedge} 5^{*} \mathrm{x} 1+$ $260969816368722240000^{*} \mathrm{y} 2^{\wedge} 2^{*} \mathrm{u}[4]^{\wedge} 7^{*} \mathrm{x} 1+782909449106166720000^{*} \mathrm{y} 2^{\wedge} 2^{*} \mathrm{u}[4]^{\wedge} 3^{*} \mathrm{x} 1$ $42952524794107353573840^{*} \mathrm{u}[4]^{\wedge} 8^{*} \mathrm{x} 1^{\wedge} 2^{*} \mathrm{y} 2-333085037439238738421760^{*} \mathrm{x} 2^{\wedge} 2^{*} \mathrm{u}[4]^{\wedge} 3^{*} \mathrm{y} 1$ $+316461166381737021901584^{*} \mathrm{x} 0^{\wedge} 2^{*} \mathrm{u}[4]^{\wedge} 7^{*} \mathrm{x} 3+$

$993373050791794726769736^{*} \mathrm{x} 0^{*} \mathrm{u}[4]^{\wedge} 6^{*} \mathrm{x} 3^{\wedge} 2-203100113433600924121044^{*} \mathrm{x} 0^{*} \mathrm{u}[4]^{\wedge} 8^{*} \mathrm{x} 3^{\wedge} 2$ - $2054753624985369997604016^{*} \mathrm{x} 0^{\wedge} 2^{*} \mathrm{u}[4]^{\wedge} 3^{*} \mathrm{x} 3-$ $1829038834156712419901616^{*} \mathrm{x} 0^{\wedge} 2^{*} \mathrm{u}[4]^{\wedge} 5^{*} \mathrm{x} 3-$ $1097983246159724796052296^{*} \mathrm{x} 0 * \mathrm{u}[4]^{\wedge} 2^{*} \mathrm{x} 3^{\wedge} 2+$ $126602728824993321465600^{*} \mathrm{x} 0{ }^{*} \mathrm{u}[4]^{\wedge} 4^{*} \mathrm{x} 3^{\wedge} 2+321959299746002834947344^{*} \mathrm{x} 0 \wedge 2^{*} \mathrm{u}[4]^{*} \mathrm{x} 3$ $+5983861486203974529108^{*} \mathrm{x} 1^{*} \mathrm{u}[4]^{\wedge} 8^{*} \mathrm{x} 22^{\wedge} 2-165279155929241391341712^{*} \mathrm{x} 1{ }^{\wedge} 2^{*} \mathrm{u}[4]^{\wedge} 7^{*} \mathrm{x} 2$ $+27263016879020187267888^{*} \mathrm{x} 1^{\wedge} 2^{*} \mathrm{u}[4]^{\wedge} 5^{*} \mathrm{x} 2+$ $300083018111910121806648^{*} \mathrm{x} 1^{*} \mathrm{u}[4]^{\wedge} 6^{*} \mathrm{x} 2^{\wedge} 2+$ $149821718207043561004800^{*} \mathrm{x} 1^{*} \mathrm{u}[4]^{\wedge} 4^{*} \mathrm{x} 22^{\wedge} 2+$ $231082537901347147871088^{*} \mathrm{x} 1^{\wedge} 2^{*} \mathrm{u}[4]^{\wedge} 3^{*} \mathrm{x} 2-184443939007593423622992^{*} \mathrm{x} 1{ }^{\wedge} 2^{*} \mathrm{u}[4]^{*} \mathrm{x} 2+$ $1127944258631582745600^{*} \mathrm{y} 0{ }^{*} \mathrm{u}[4]^{\wedge} 8^{*} \mathrm{y} 2{ }^{*} \mathrm{x} 2+36650394224513165491200^{*} \mathrm{y} 0 * \mathrm{u}[4]^{\wedge} 6^{*} \mathrm{y} 2{ }^{*} \mathrm{x} 2$ - 36650394224513165491200* $0^{*} \mathrm{u}[4]^{\wedge} 2^{*} \mathrm{y} 2^{*} \mathrm{x} 2+181706575495470105600^{*} \mathrm{u}[4]^{\wedge} 8^{*} \mathrm{y} 3{ }^{*} \mathrm{y} 1{ }^{*} \mathrm{x} 2$ $-34031092556259059788800^{*} \mathrm{u}[4]^{\wedge} 6^{*} \mathrm{y} 3^{*} \mathrm{y} 1^{*} \mathrm{x} 2-$ $34535483262877052851200 * \mathrm{y} 0 * \mathrm{y} 1 * \mathrm{x} 1 * \mathrm{u}[4]^{\wedge} 6+$ $34535483262877052851200^{*} \mathrm{y} 0^{*} \mathrm{y} 1^{*} \mathrm{x} 1^{*} \mathrm{u}[4]^{\wedge} 2-70488777813526425600^{*} \mathrm{y} 0^{*} \mathrm{y} 1^{*} \mathrm{x} 1^{*} \mathrm{u}[4]^{\wedge} 8-$ $11790246517075442249280^{*} \mathrm{x} 2^{*} \mathrm{u}[4]^{\wedge} 3^{*} \mathrm{y} 0 * \mathrm{x} 1-6546365434194168960000^{*} \mathrm{u}[4]^{\wedge} 5^{*} \mathrm{y} 1{ }^{*} \mathrm{y} 2{ }^{*} \mathrm{x} 1-$ $2182121811398056320000^{*} \mathrm{u}[4]^{*} \mathrm{y} 1^{*} \mathrm{y} 2^{*} \mathrm{x} 1-79301509407336702266778^{*} \mathrm{x} 1^{\wedge} 3^{*} \mathrm{u}[4]^{\wedge} 7+$ $130325477752378454680122^{*} \times 1^{\wedge} 3^{*} u[4]^{\wedge} 5-86292906623260195047078^{*} x 1^{\wedge} 3^{*} u[4]^{\wedge} 3-$ $58644876004763233057812^{*} \mathrm{u}[4]^{\wedge} 6^{*} \mathrm{x} 2^{\wedge} 3+323071816158356092732800^{*} \mathrm{u}[4]^{\wedge} 4^{*} \mathrm{x} 2^{\wedge} 3+$ $79193258199265968915732^{*} \mathrm{u}[4]^{\wedge} 2^{*} \mathrm{x} 22^{\wedge} 3-665809932708633883357116^{*} \mathrm{x} 1^{*} \mathrm{u}[4]^{\wedge} 5^{*} \mathrm{x} 2^{\wedge} 2+$ $252034560280177555196484^{*} \mathrm{x} 1^{*} \mathrm{u}[4]^{\wedge} 3^{*} \mathrm{x} 2^{\wedge} 2+66889813126305175513044^{*} \mathrm{x} 1^{*} \mathrm{u}[4]^{*} \mathrm{x} 2^{\wedge} 2+$ $397151671246914078495186^{*} \mathrm{x} 1^{\wedge} 2^{*} \mathrm{u}[4]^{\wedge} 6^{*} \mathrm{x} 2-433236768751277299454400^{*} \mathrm{x} 1^{\wedge} 2^{*} \mathrm{u}[4]^{\wedge} 4^{*} \mathrm{x} 2$ $-3647982971736042102720^{*} \mathrm{u}[4]^{*} \mathrm{x} 2^{\wedge} 2^{*} \mathrm{y} 3-25936793211446312505840^{*} \mathrm{u}[4]^{\wedge} 7^{*} \mathrm{x} 1{ }^{*} \mathrm{y} 2^{*} \mathrm{x} 3+$ $411068038279031030836080^{*} \mathrm{x} 2{ }^{*} \mathrm{u}[4]^{\wedge} 3^{*} \mathrm{y} 1{ }^{*} \mathrm{x} 3-6840407029286969049600^{*} \mathrm{u}[4]^{\wedge} 5^{*} \mathrm{y} 1{ }^{*} \mathrm{y} 2{ }^{*} \mathrm{x} 3$ 
- $151440993753907435920^{*} \mathrm{u}[4]^{\wedge} 3^{*} \mathrm{x} 1^{*} \mathrm{y} 2{ }^{*} \mathrm{x} 3+3204390312071010316800^{*} \mathrm{u}[4]^{*} \mathrm{y} 1{ }^{*} \mathrm{y} 2{ }^{*} \mathrm{x} 3+$ $116284248070782785318160^{*} \mathrm{x} 2^{*} \mathrm{u}[4]^{\wedge} 7^{*} \mathrm{y} 1^{*} \mathrm{x} 3-8288995029277204569600^{*} \mathrm{x} 1^{*} \mathrm{u}[4]^{\wedge} 4^{*} \mathrm{y} 1^{*} \mathrm{x} 3$ $+141185880392275746531360 * \mathrm{u}[4]^{\wedge} 6{ }^{*} \mathrm{x} 2{ }^{*} \mathrm{y} 2 * \mathrm{x} 3+$

$1278424537921733529600^{*} \mathrm{u}[4]^{\wedge} 4^{*} \mathrm{x} 2^{*} \mathrm{y} 2{ }^{*} \mathrm{x} 3-148134244837104013001760^{*} \mathrm{u}[4]^{\wedge} 2^{*} \mathrm{x} 2{ }^{*} \mathrm{y} 2{ }^{*} \mathrm{x} 3$ $+28975728014303829575760 * \mathrm{x} 1 * \mathrm{u}[4]^{\wedge} 2^{*} \mathrm{y} 1 * \mathrm{x} 3+$ $111730145950575681042960 * \mathrm{u}[4]^{*} \mathrm{x} 2^{*} \mathrm{y} 1{ }^{*} \mathrm{x} 3-589398044823556300800^{*} \mathrm{y} 2^{\wedge} 2^{*} \mathrm{u}[4]^{\wedge} 8^{*} \mathrm{x} 3+$ $5292190581022112601600^{*} \mathrm{y} 2^{\wedge} 2^{*} \mathrm{u}[4]^{\wedge} 2^{*} \mathrm{x} 3-1163244578015311459200^{*} \mathrm{u}[4]^{\wedge} 8^{*} \mathrm{y} 11^{\wedge} 2^{*} \mathrm{x} 3+$ $13107237855702243309360^{*} \mathrm{u}[4]^{\wedge} 7^{*} \mathrm{x} 00^{\wedge} 2^{*} \mathrm{y} 1-450353161682226877443120^{*} \mathrm{u}[4]^{\wedge} 5^{*} \mathrm{x} 00^{\wedge} 2^{*} \mathrm{y} 1-$ $459373567423764024886320^{*} \mathrm{u}[4]^{\wedge} 3^{*} \mathrm{x} 0^{\wedge} 2^{*} \mathrm{y} 1-25617269986436514350880^{*} \mathrm{u}[4]^{\wedge} 8^{*} \mathrm{x} 0^{\wedge} 2^{*} \mathrm{y} 2$ $+269791910343122918400^{*} \mathrm{u}[4]^{*} \mathrm{y} 2^{\wedge} 2^{*} \mathrm{x} 0-30130524215343533426400^{*} \mathrm{u}[4]^{*} \mathrm{x} 3^{*} \mathrm{y} 0{ }^{*} \mathrm{x} 2+$ $131388309321752487600000^{*} \mathrm{u}[4]^{\wedge} 2^{*} \mathrm{x} 3^{*} \mathrm{y} 0^{*} \mathrm{x} 1-67976980881234112659600^{*} \mathrm{x} 0{ }^{*} \mathrm{u}[4]^{*} \mathrm{y} 0^{*} \mathrm{x} 1$ $+89983369980484699683600^{*} \mathrm{u}[4]^{*} \mathrm{x} 3^{*} \mathrm{y} 3^{*} \mathrm{x} 1+3025626135112824796800^{*} \mathrm{u}[4]^{*} \mathrm{y} 0^{\wedge} 2^{*} \mathrm{x} 1+$ $3413625284728554854400^{*} \mathrm{u}[4]^{\wedge} 5^{*} \mathrm{y} 0^{*} \mathrm{y} 3^{*} \mathrm{x} 1-3536818019090481715200^{*} \mathrm{u}[4]^{*} \mathrm{y} 0^{*} \mathrm{y} 3^{*} \mathrm{x} 1-$ $298178007522184027942560^{*} \mathrm{u}[4]^{\wedge} 6^{*} \mathrm{x} 0^{\wedge} 2^{*} \mathrm{y} 2-7421535569013038553600^{*} \mathrm{u}[4]^{\wedge} 4^{*} \mathrm{x} 00^{\wedge} 2^{*} \mathrm{y} 2+$ $302378331996795989388960^{*} \mathrm{u}[4]^{\wedge} 2^{*} \mathrm{x} 0^{\wedge} 2^{*} \mathrm{y} 2+15708692157790095866160^{*} \mathrm{u}[4]^{*} \mathrm{x} 00^{\wedge} 2^{*} \mathrm{y} 1$ $175353703479381384045360^{*} \mathrm{x} 3^{\wedge} 2^{*} \mathrm{u}[4]^{\wedge} 7^{*} \mathrm{y} 1-34590083660322961484880^{*} \mathrm{x} 3^{\wedge} 2^{*} \mathrm{u}[4]^{\wedge} 5^{*} \mathrm{y} 1-$ $23773526390298230761680^{*} \mathrm{x} 3^{\wedge} 2^{*} \mathrm{y} 1^{*} \mathrm{u}[4]^{\wedge} 3-55056924943281160197120^{*} \mathrm{x} 3^{\wedge} 2^{*} \mathrm{u}[4]^{\wedge} 8^{*} \mathrm{y} 2+$ $138625769191236262126560^{*} \mathrm{x} 3^{\wedge} 2^{*} \mathrm{u}[4]^{\wedge} 6^{*} \mathrm{y} 2+11013838625988205113600^{*} \mathrm{x} 3^{\wedge} 2^{*} \mathrm{u}[4]^{\wedge} 4^{*} \mathrm{y} 2$ $-2182121811398056320000^{*} \mathrm{u}[4]^{\wedge} 7^{*} \mathrm{y} 1{ }^{*} \mathrm{y} 2^{*} \mathrm{x} 1+1091060905699028160000^{*} \mathrm{u}[4]^{\wedge} 8^{*} \mathrm{y} 1{ }^{*} \mathrm{y} 2{ }^{*} \mathrm{x} 2$ $+2182121811398056320000^{*} \mathrm{u}[4]^{\wedge} 6^{*} \mathrm{y} 1^{*} \mathrm{y} 2^{*} \mathrm{x} 2-6546365434194168960000^{*} \mathrm{u}[4]^{\wedge} 3^{*} \mathrm{y} 1^{*} \mathrm{y} 2^{*} \mathrm{x} 1$ $-24796706812725269771520^{*} \mathrm{x} 0 * \mathrm{u}[4]^{\wedge} 7^{*} \mathrm{y} 0 * \mathrm{x} 3+$

$3505285245677735520000^{*} \mathrm{y} 0 * \mathrm{u}[4]^{\wedge} 7^{*} \mathrm{y} 3^{*} \mathrm{x} 3+10515855737033206560000^{*} \mathrm{y} 0 * \mathrm{u}[4]^{\wedge} 5^{*} \mathrm{y} 3^{*} \mathrm{x} 3$ $+10515855737033206560000^{*} \mathrm{y} 0^{*} \mathrm{u}[4]^{\wedge} 3^{*} \mathrm{y} 3 * \mathrm{x} 3-$

$3505285245677735520000^{*} \mathrm{y} 0 * \mathrm{y} 3 * \mathrm{u}[4]^{\wedge} 6{ }^{*} \mathrm{x} 0+76555832064922324034880^{*} \mathrm{u}[4]^{\wedge} 7^{*} \mathrm{y} 2{ }^{*} \mathrm{x} 3{ }^{*} \mathrm{x} 2$ $-152034795997200117552960 * \mathrm{u}[4]^{\wedge} 5^{*} \mathrm{y} 2{ }^{*} \mathrm{x} 33^{*} \mathrm{x} 2-$

$105095792026478501386560^{*} \mathrm{u}[4]^{\wedge} 3^{*} \mathrm{y} 2 * \mathrm{x} 3^{*} \mathrm{x} 2-$

$129296844536610177520320^{*} \mathrm{y} 1^{*} \mathrm{u}[4]^{\wedge} 2^{*} \mathrm{x} 3^{*} \mathrm{x} 2+$

$176580825219085170775680^{*} \mathrm{u}[4]^{\wedge} 2^{*} \mathrm{y} 2^{*} \mathrm{x} 0 * \mathrm{x} 2$

$102546085071404358722880^{*} \mathrm{y} 1^{*} \mathrm{u}[4]^{\wedge} 7^{*} \mathrm{x} 3^{*} \mathrm{x} 1-$

$12575625608510070733440^{*} \mathrm{u}[4]^{\wedge} 3^{*} \mathrm{y} 2^{*} \mathrm{x} 0{ }^{*} \mathrm{x} 1-93176862486795655027200^{*} \mathrm{u}[4]^{\wedge} 55^{*} \mathrm{y} 1{ }^{*} \mathrm{y} 2{ }^{*} \mathrm{x} 0$ $+14800386780734781657600^{*} \mathrm{u}[4]^{*} \mathrm{y} 1{ }^{*} \mathrm{y} 2^{*} \mathrm{x} 0-31445772312431266580160^{*} \mathrm{y} 0 * \mathrm{u}[4]^{\wedge} 7^{*} \mathrm{x} 2{ }^{*} \mathrm{x} 1$ $+20393739204465737255040^{*} \mathrm{y} 0^{*} \mathrm{x} 1^{\wedge} 2^{*} \mathrm{u}[4]+4350286933367038410960^{*} \mathrm{y} 0^{*} \mathrm{u}[4]^{*} \mathrm{x} 2^{\wedge} 2+$ $4364243622796112640000^{*} \mathrm{u}[4]^{\wedge} 4^{*} \mathrm{y} 2^{*} \mathrm{x} 1{ }^{*} \mathrm{x} 2+67817021106466350296640^{*} \mathrm{u}[4]^{\wedge} 2^{*} \mathrm{y} 2{ }^{*} \mathrm{x} 1{ }^{*} \mathrm{x} 2$ $+27379787705922767002560^{*} \mathrm{y} 1^{*} \mathrm{x} 1^{*} \mathrm{u}[4]^{*} \mathrm{x} 2-8728487245592225280000^{*} \mathrm{y} 1^{*} \mathrm{u}[4]^{\wedge} 4^{*} \mathrm{x} 2^{\wedge} 2-$ $9201505240894948937280^{*} \mathrm{u}[4]^{\wedge} 7^{*} \mathrm{y} 2^{*} \mathrm{x} 2^{\wedge} 2+16982661266953975961280^{*} \mathrm{u}[4]^{\wedge} 7^{*} \mathrm{y} 2^{*} \mathrm{x} 1^{\wedge} 2+$ $48905707455606207136320^{*} \mathrm{u}[4]^{\wedge} 5^{*} \mathrm{y} 2{ }^{*} \mathrm{x} 2 \wedge 2-19015873943234957104320^{*} \mathrm{u}[4]^{\wedge} 5^{*} \mathrm{y} 2{ }^{*} \mathrm{x} 1 \wedge 2+$ $51087829267004263456320^{*} \mathrm{u}[4]^{\wedge} 3^{*} \mathrm{y} 2^{*} \mathrm{x} 22^{\wedge} 2-14651630320438844464320^{*} \mathrm{u}[4]^{\wedge} 3^{*} \mathrm{y} 2^{*} \mathrm{x} 1^{\wedge} 2$ $7019383429496892617280^{*} \mathrm{u}[4]^{*} \mathrm{y} 2^{*} \mathrm{x} 22^{\wedge} 2+59892165497469469132800^{*} \mathrm{u}[4]^{\wedge} 3^{*} \mathrm{y} 3^{*} \mathrm{y} 1{ }^{*} \mathrm{x} 0+$ $22301401722823156377600^{*} \mathrm{u}[4]^{*} \mathrm{y} 3^{*} \mathrm{y} 1^{*} \mathrm{x} 0+7701904786857807052800^{*} \mathrm{y} 0^{*} \mathrm{u}[4]^{\wedge} 5^{*} \mathrm{y} 2^{*} \mathrm{x} 0+$ $10258796810741337331200^{*} \mathrm{u}[4]^{\wedge} 8^{*} \mathrm{y} 3^{*} \mathrm{y} 2^{*} \mathrm{x} 0+$

$17011573785982674662400^{*} \mathrm{u}[4]^{\wedge} 6^{*} \mathrm{y} 3^{*} \mathrm{y} 2^{*} \mathrm{x} 0-17011573785982674662400^{*} \mathrm{u}[4]^{\wedge} 2^{*} \mathrm{y} 3^{*} \mathrm{y} 2^{*} \mathrm{x} 0$ - $19652510479298385561600 * \mathrm{u}[4]^{\wedge} 6^{*} \mathrm{y} 3 * \mathrm{x} 2 * \mathrm{x} 0-$

$43806176025531163545600^{*} \mathrm{u}[4]^{\wedge} 4^{*} \mathrm{y} 3^{*} \mathrm{x} 2^{*} \mathrm{x} 0-17141625875232777984000^{*} \mathrm{u}[4]^{\wedge} 2^{*} \mathrm{y} 3^{*} \mathrm{x} 2{ }^{*} \mathrm{x} 0$ $+8699528618909085801600^{*} \mathrm{y} 0^{*} \mathrm{u}[4]^{\wedge} 6^{*} \mathrm{x} 1^{*} \mathrm{x} 0-1165982503489584336000^{*} \mathrm{y} 0^{*} \mathrm{u}[4]^{\wedge} 2^{*} \mathrm{x} 1^{*} \mathrm{x} 0$ - $1663237016800903180800^{*} \mathrm{y} 0 * \mathrm{u}[4]^{*} \mathrm{x} 2{ }^{*} \mathrm{x} 0-10274195902536578188800^{*} \mathrm{y} 0 * \mathrm{u}[4]^{\wedge} 8^{*} \mathrm{y} 2{ }^{*} \mathrm{x} 3-$ 
$24054411640573156377600 * \mathrm{y} 0 * \mathrm{u}[4]^{\wedge} 6 * \mathrm{y} 2 * \mathrm{x} 3+$ $24054411640573156377600^{*} \mathrm{y} 0 * \mathrm{u}[4]^{\wedge} 2^{*} \mathrm{y} 2{ }^{*} \mathrm{x} 3-991482478184634508800^{*} \mathrm{u}[4]^{\wedge} 8^{*} \mathrm{y} 3{ }^{*} \mathrm{y} 1{ }^{*} \mathrm{x} 3+$ $1523054879130730982400^{*} \mathrm{u}[4]^{\wedge} 6^{*} \mathrm{y} 3{ }^{*} \mathrm{y} 1{ }^{*} \mathrm{x} 3-1523054879130730982400^{*} \mathrm{u}[4]^{\wedge} 2^{*} \mathrm{y} 3{ }^{*} \mathrm{y} 1{ }^{*} \mathrm{x} 3+$ $12459432458817020790720^{*} \mathrm{u}[4]^{*} \mathrm{y} 3^{*} \mathrm{x} 1^{\wedge} 2-66074708002442837491200^{*} \mathrm{x} 0 \wedge 3^{*} \mathrm{u}[4]^{\wedge} 4+$ $652444391394340197190032^{*} \mathrm{x} 0^{\wedge} 3^{*} \mathrm{u}[4]^{\wedge} 2-399315421707962946817128^{*} \mathrm{u}[4]^{\wedge} 7^{*} \mathrm{x} 3^{\wedge} 3$ $280922505271455393563928^{*} \mathrm{u}[4]^{\wedge} 5^{*} \mathrm{x} 3^{\wedge} 3-217621140858958732831128^{*} \mathrm{u}[4]^{\wedge} 3^{*} \mathrm{x} 3^{\wedge} 3$ $413085032026440749667048^{*} \mathrm{u}[4]^{*} \mathrm{x} 33^{\wedge} 3-641448124665808571098512^{*} \mathrm{x} 0^{\wedge} 3^{*} \mathrm{u}[4]^{\wedge} 6+$ $81196232112563960664000 * \mathrm{u}[4]^{\wedge} 5 * \mathrm{y} 3 * \mathrm{x} 1{ }^{*} \mathrm{x} 0+$ $14793573252769374240000^{*} \mathrm{x} 2^{*} \mathrm{u}[4]^{\wedge} 5^{*} \mathrm{y} 0^{*} \mathrm{x} 0-3705176690528787302400^{*} \mathrm{y} 0^{*} \mathrm{y} 1^{*} \mathrm{x} 00^{*} \mathrm{u}[4]^{\wedge} 6$ $+3705176690528787302400 * \mathrm{y} 0 * \mathrm{y} 1{ }^{*} \mathrm{x} 0 * \mathrm{u}[4]^{\wedge} 2-99578427514393651200^{*} \mathrm{y} 0 * \mathrm{y} 1{ }^{*} \mathrm{x} 0 * \mathrm{u}[4]^{\wedge} 8+$ $57632528732819824036800^{*} \mathrm{u}[4]^{\wedge} 3^{*} \mathrm{y} 3^{*} \mathrm{x} 1^{*} \mathrm{x} 0+$ $39064820927201189966400^{*} \mathrm{u}[4]^{\wedge} 7^{*} \mathrm{y} 3^{*} \mathrm{x} 1^{*} \mathrm{x} 0+$ $21637254252331068652800^{*} \mathrm{x} 2^{*} \mathrm{u}[4]^{\wedge} 3^{*} \mathrm{y} 0^{*} \mathrm{x} 0-1494878345362597593600^{*} \mathrm{y} 0 * \mathrm{u}[4]^{\wedge} 7^{*} \mathrm{x} 2^{*} \mathrm{x} 0$ $+1279279299508405292160^{*} \mathrm{x} 2^{\wedge} 2^{*} \mathrm{u}[4]^{\wedge} 8^{*} \mathrm{y} 0+39557559172242230997120^{*} \mathrm{x} 2^{\wedge} 2^{*} \mathrm{u}[4]^{\wedge} 6^{*} \mathrm{y} 0$ - $72855917258298556262400^{*} \mathrm{x} 2{ }^{\wedge} 2^{*} \mathrm{u}[4]^{\wedge} 4^{*} \mathrm{y} 0-2182121811398056320000^{*} \mathrm{y} 2^{\wedge} 2^{*} \mathrm{x} 1^{*} \mathrm{u}[4]^{\wedge} 6+$ $2182121811398056320000^{*} \mathrm{y} 22^{\wedge} 2^{*} \mathrm{x} 1^{*} \mathrm{u}[4]^{\wedge} 2-1091060905699028160000^{*} \mathrm{y} 2^{\wedge} 2^{*} \mathrm{x} 1^{*} \mathrm{u}[4]^{\wedge} 8+$ $2182121811398056320000^{*} \mathrm{u}[4]^{\wedge} 7^{*} \mathrm{y} 1^{\wedge} 2^{*} \mathrm{x} 2+6546365434194168960000^{*} \mathrm{u}[4]^{\wedge} 5^{*} \mathrm{y} 1^{\wedge} 2^{*} \mathrm{x} 2+$ $6546365434194168960000^{*} \mathrm{u}[4]^{\wedge} 3^{*} \mathrm{y} 1^{\wedge} 2^{*} \mathrm{x} 2+264706217793813116160{ }^{*} \mathrm{y} 1{ }^{*} \mathrm{x} 0{ }^{\wedge} 2-$ $7843268600165418531840^{*} \mathrm{y} 0 * \mathrm{x} 1{ }^{\wedge} 2-60080562103287398400^{*} \mathrm{y} 3^{\wedge} 2^{*} \mathrm{x} 2+$ $5235790503263664314880^{*} \mathrm{x} 1^{\wedge} 2^{*} \mathrm{y} 1+3413625284728554854400^{*} \mathrm{u}[4]^{\wedge} 3^{*} \mathrm{y} 0{ }^{*} \mathrm{y} 3^{*} \mathrm{x} 1$ $7042837854590481715200^{*} \mathrm{u}[4]^{\wedge} 2^{*} \mathrm{y} 0^{*} \mathrm{y} 3^{*} \mathrm{x} 2-3705176690528787302400^{*} \mathrm{y} 0^{\wedge} 2^{*} \mathrm{x} 1^{*} \mathrm{u}[4]^{\wedge} 2+$ $99578427514393651200^{*} \mathrm{y} 0 \wedge 2^{*} \mathrm{x} 1^{*} \mathrm{u}[4]^{\wedge} 8-229955038619269017600^{*} \mathrm{y} 0^{\wedge} 2^{*} \mathrm{u}[4]^{\wedge} 7^{*} \mathrm{x} 2-$ $7701904786857807052800^{*} \mathrm{y} 0^{\wedge} 2^{*} \mathrm{u}[4]^{\wedge} 5^{*} \mathrm{x} 2-7701904786857807052800^{*} \mathrm{y}^{\wedge} 2^{*} \mathrm{u}[4]^{\wedge} 3^{*} \mathrm{x} 2-$ $229955038619269017600^{*} \mathrm{y} 0^{\wedge} 2^{*} \mathrm{u}[4]^{*} \mathrm{x} 2-6344732884908337920000^{*} \mathrm{y} 0 * \mathrm{u}[4]^{\wedge} 3^{*} \mathrm{y} 3^{*} \mathrm{x} 0-$ $6344732884908337920000^{*} \mathrm{y} 0 * \mathrm{y} 3^{*} \mathrm{u}[4]^{\wedge} 5^{*} \mathrm{x} 0-2114910961636112640000^{*} \mathrm{y} 0{ }^{*} \mathrm{y} 3 * \mathrm{u}[4]^{\wedge} 6^{*} \mathrm{x} 3-$ $2114910961636112640000^{*} \mathrm{y} 0 * \mathrm{u}[4]^{\wedge} 7^{*} \mathrm{y} 3^{*} \mathrm{x} 0-2114910961636112640000^{*} \mathrm{y} 0{ }^{*} \mathrm{y} 3^{*} \mathrm{u}[4]^{*} \mathrm{x} 0-$ $250767067603269146366814^{*} \mathrm{x} 0^{\wedge} 3^{*} \mathrm{u}[4]-326610415235777830888254^{*} \mathrm{x} 0^{\wedge} 3^{*} \mathrm{u}[4]^{\wedge} 7+$ $336043421664027392709246^{*} \mathrm{x} 0^{\wedge} 3^{*} \mathrm{u}[4]^{\wedge} 5+436764998564399238575646^{*} \mathrm{x} 0^{\wedge} 3^{*} \mathrm{u}[4]^{\wedge} 3-$ $12652594242545945316864^{*} \mathrm{u}[4]^{\wedge} 8^{*} \mathrm{x} 3^{\wedge} 3-57299306881059208650252^{*} \mathrm{u}[4]^{\wedge} 8^{*} \mathrm{x} 33^{*} \mathrm{x} 1{ }^{*} \mathrm{x} 2+$ $142342518513114424740480 * \mathrm{y} 1 * \mathrm{x} 0 * \mathrm{x} 1 * \mathrm{u}[4]^{\wedge} 6-$ $151648027693421192407680^{*} \mathrm{y} 1^{*} \mathrm{x} 0^{*} \mathrm{x} 1^{*} \mathrm{u}[4]^{\wedge} 2-3410850463835959010880^{*} \mathrm{y} 1^{*} \mathrm{x} 0{ }^{*} \mathrm{u}[4]^{\wedge} 7^{*} \mathrm{x} 2$ - $369439467877451536943040^{*} \mathrm{y} 1{ }^{*} \mathrm{x} 0 * \mathrm{u}[4]^{\wedge} 5^{*} \mathrm{x} 2$ -

$300005088200706491029440^{*} \mathrm{y} 1^{*} \mathrm{x} 00^{*} \mathrm{u}[4]^{\wedge} 3^{*} \mathrm{x} 2-2765482201590913097280 * \mathrm{y} 1{ }^{*} \mathrm{x} 0{ }^{*} \mathrm{u}[4]^{*} \mathrm{x} 2+$ $71949126016117900848960 * \mathrm{u}[4]^{\wedge} 5^{*} \mathrm{y} 1{ }^{*} \mathrm{x} 3{ }^{*} \mathrm{x} 1+$ $211176315863961395747520^{*} \mathrm{u}[4]^{\wedge} 6^{*} \mathrm{y} 1^{*} \mathrm{x} 3^{*} \mathrm{x} 2+$ $13090459912851218227200 * \mathrm{u}[4]^{\wedge} 4^{*} \mathrm{y} 1 * \mathrm{x} 3 * \mathrm{x} 2$ $1196713309085462443788312^{*} \mathrm{x} 0 * \mathrm{u}[4]^{\wedge} 5 * \mathrm{x} 1{ }^{*} \mathrm{x} 2-$ $393127238593090951168152^{*} \mathrm{u}[4]^{\wedge} 7^{*} \mathrm{x} 3^{*} \mathrm{x} 1^{\wedge} 2+$ $438439683910664989639152 * \mathrm{x} 0 * \mathrm{u}[4]^{\wedge} 6^{*} \mathrm{x} 1^{\wedge} 2-113798171863745408092800 * \mathrm{x} 0 * \mathrm{u}[4]^{\wedge} 4^{*} \mathrm{x} 1 \wedge 2$ - $664839759837653697287952^{*} \mathrm{x} 0^{*} \mathrm{u}[4]^{\wedge} 6^{*} \mathrm{x} 2^{\wedge} 2+$ $261228583810579205260800^{*} \mathrm{x} 0 * \mathrm{u}[4]^{\wedge} 4^{*} \mathrm{x} 22^{\wedge} 2+66645455499061620923352^{*} \mathrm{u}[4]^{\wedge} 7^{*} \mathrm{x} 3{ }^{*} \mathrm{x} 2{ }^{\wedge} 2$ $-5931091146019074796800^{*} \mathrm{u}[4]^{\wedge} 6^{*} \mathrm{y} 2^{*} \mathrm{y} 3^{*} \mathrm{x} 3+5931091146019074796800^{*} \mathrm{u}[4]^{\wedge} 2^{*} \mathrm{y} 2{ }^{*} \mathrm{y} 3^{*} \mathrm{x} 3$ - 3025626135112824796800* $1^{*} \mathrm{u}[4]^{*} \mathrm{y} 0{ }^{*} \mathrm{x} 0-2100162237305980723200^{*} \mathrm{u}[4]^{\wedge} 7^{*} \mathrm{y} 2^{*} \mathrm{y} 3^{*} \mathrm{x} 0+$ $5321373331707057830400 * \mathrm{u}[4]^{\wedge} 5^{*} \mathrm{y} 2{ }^{*} \mathrm{y} 3{ }^{*} \mathrm{x} 0+5321373331707057830400 * \mathrm{u}[4]^{\wedge} 3^{*} \mathrm{y} 2{ }^{*} \mathrm{y} 3{ }^{*} \mathrm{x} 0-$ $2100162237305980723200^{*} \mathrm{u}[4]^{*} \mathrm{y} 2^{*} \mathrm{y} 3^{*} \mathrm{x} 0-438756976680209133472080^{*} \mathrm{x} 3^{*} \mathrm{y} 1^{*} \mathrm{u}[4]^{\wedge} 2^{*} \mathrm{x} 0+$ 
$6192060962387797036800 * \mathrm{u}[4]^{\wedge} 6^{*} \mathrm{y} 2^{*} \mathrm{y} 0 * \mathrm{x} 0+$ $183651770839388717591280^{*} \mathrm{x} 3{ }^{*} \mathrm{u}[4]^{\wedge} 7^{*} \mathrm{y} 2 * \mathrm{x} 0-$ $434098638649458638915760 * \mathrm{x} 3 * \mathrm{u}[4]^{\wedge} 5 * \mathrm{y} 2 * \mathrm{x} 0-$ $457843650801490510749360^{*} \mathrm{x} 3^{*} \mathrm{u}[4]^{\wedge} 3^{*} \mathrm{y} 2^{*} \mathrm{x} 0+402651386800134638400^{*} \mathrm{y} 1 * \mathrm{u}[4]^{\wedge} 8^{*} \mathrm{y} 0 * \mathrm{x} 3$ $-5005627248212230723200^{*} \mathrm{y} 1{ }^{*} \mathrm{u}[4]^{\wedge} 6^{*} \mathrm{y} 0 * \mathrm{x} 3+5005627248212230723200^{*} \mathrm{y} 1^{*} \mathrm{u}[4]^{\wedge} 2^{*} \mathrm{y} 0 * \mathrm{x} 3$ - 60080562103287398400*u[4]^ $8^{*} \mathrm{y} 2^{*} \mathrm{y} 3^{*} \mathrm{x} 3+3286595951481547036800^{*} \mathrm{y} 1^{*} \mathrm{y} 3{ }^{*} \mathrm{u}[4]^{\wedge} 7^{*} \mathrm{x} 3-$ $1762072189180358889600^{*} \mathrm{y} 1^{*} \mathrm{y} 3^{*} \mathrm{u}[4]^{\wedge} 5^{*} \mathrm{x} 3-1762072189180358889600^{*} \mathrm{y} 1^{*} \mathrm{y} 3^{*} \mathrm{u}[4]^{\wedge} 3^{*} \mathrm{x} 3+$ $3286595951481547036800^{*} \mathrm{y} 1^{*} \mathrm{y} 3^{*} \mathrm{u}[4]^{*} \mathrm{x} 3-304010708818397443200^{*} \mathrm{u}[4]^{\wedge} 7^{*} \mathrm{y} 2^{*} \mathrm{y} 0 * \mathrm{x} 3+$ $10709827917169807670400 * \mathrm{u}[4]^{\wedge} 5{ }^{*} \mathrm{y} 2 * \mathrm{y} 0 * \mathrm{x} 3+$ $10709827917169807670400 * \mathrm{u}[4]^{\wedge} 3^{*} \mathrm{y} 2{ }^{*} \mathrm{y} 0 * \mathrm{x} 3-3025626135112824796800 * \mathrm{y} 1^{*} \mathrm{u}[4]^{\wedge} 7^{*} \mathrm{y} 0{ }^{*} \mathrm{x} 0$ $+2544981638286525609600^{*} \mathrm{y} 1^{*} \mathrm{u}[4]^{\wedge} 3^{*} \mathrm{y} 0 * \mathrm{x} 0+$ $2544981638286525609600^{*} \mathrm{y} 1 * \mathrm{u}[4]^{\wedge} 5^{*} \mathrm{y} 00^{*} \mathrm{x} 0+1952166772778787302400 * \mathrm{u}[4]^{*} \mathrm{y} 2{ }^{*} \mathrm{y} 3 * \mathrm{x} 3-$ $1523054879130730982400^{*} \mathrm{y} 3^{\wedge} 2^{*} \mathrm{u}[4]^{\wedge} 6^{*} \mathrm{x} 1-1952166772778787302400^{*} \mathrm{y} 3^{\wedge} 2^{*} \mathrm{u}[4]^{\wedge} 7^{*} \mathrm{x} 2+$ $1155539352663638092800^{*} \mathrm{y} 3^{\wedge} 2^{*} \mathrm{u}[4]^{\wedge} 5^{*} \mathrm{x} 2+1155539352663638092800^{*} \mathrm{y} 3^{\wedge} 2^{*} \mathrm{u}[4]^{\wedge} 3^{*} \mathrm{x} 2-$ $8901855626342312952000^{*} \mathrm{u}[4]^{\wedge} 2^{*} \mathrm{x} 3^{*} \mathrm{y}^{*} \mathrm{x} 1+5592334650591726523200 * \mathrm{u}[4]^{*} \mathrm{x} 3{ }^{*} \mathrm{y} 0{ }^{*} \mathrm{x} 1-$ $206890164254980442206127^{*} \mathrm{u}[4]^{\wedge} 8^{*} \mathrm{x} 3^{*} \mathrm{x} 0 * \mathrm{x} 1+$ $356681752218806867379206 * \mathrm{u}[4]^{\wedge} 7^{*} \mathrm{x} 3^{*} \mathrm{x} 0 * \mathrm{x} 2-$ $316261520926343955404640^{*} \mathrm{x} 0^{*} \mathrm{x} 3^{*} \mathrm{u}[4]^{\wedge} 4^{*} \mathrm{x} 1-$ $2130607662929803440834702 * \mathrm{x} 0 * \mathrm{x} 3 * \mathrm{u}[4]^{\wedge} 5 * \mathrm{x} 2-$ $1820666079601650406577022^{*} \mathrm{x} 0^{*} \mathrm{x} 3^{*} \mathrm{u}[4]^{\wedge} 3^{*} \mathrm{x} 2+$ $292165460513899892068886 * \mathrm{x} 0 * \mathrm{x} 33^{*} \mathrm{u}[4]^{*} \mathrm{x} 2+$ $1093679742866260667775626^{*} \mathrm{u}[4]^{\wedge} 6^{*} \mathrm{x} 3^{*} \mathrm{x} 0^{*} \mathrm{x} 1-$ $1035483388759544613612266^{*} \mathrm{x} 0 * \mathrm{x} 3{ }^{*} \mathrm{u}[4]^{\wedge} 2^{*} \mathrm{x} 1-$ $1752642622838867760000^{*} \mathrm{y} 0 * \mathrm{u}[4]^{\wedge} 8^{*} \mathrm{y}^{*} \mathrm{x} 0-11677783830128078587200 * \mathrm{u}[4]^{*} \mathrm{x} 3{ }^{*} \mathrm{y} 3{ }^{*} \mathrm{x} 2-$ $24256496353608866295216^{*} \mathrm{x} 1^{\wedge} 3^{*} \mathrm{u}[4]^{\wedge} 6+33132832235538884266464^{*} \mathrm{x} 1^{\wedge} 3^{*} \mathrm{u}[4]^{\wedge} 8+$ $62586062510312930857776 * x 1^{\wedge} 3^{*} \mathrm{u}[4]^{\wedge} 2-35998535210188933065600^{*} \mathrm{x} 1^{\wedge} 3^{*} \mathrm{u}[4]^{\wedge} 4-$ $11676344104093557621096^{*} \mathrm{u}[4]^{\wedge} 7^{*} \mathrm{x} 2^{\wedge} 3-213457383548744178502296^{*} \mathrm{u}[4]^{\wedge} 5^{*} \mathrm{x} 2^{\wedge} 3$ $521939632737444480000^{*} \mathrm{x} 2{ }^{*} \mathrm{u}[4]^{\wedge} 4^{*} \mathrm{y} 1{ }^{*} \mathrm{x} 1-260969816368722240000^{*} \mathrm{u}[4]^{\wedge} 6{ }^{*} \mathrm{y} 1{ }^{*} \mathrm{y} 2{ }^{*} \mathrm{x} 1-$ $260969816368722240000^{*} \mathrm{u}[4]^{\wedge} 7^{*} \mathrm{y} 1^{*} \mathrm{y} 2^{*} \mathrm{x} 2-130484908184361120000^{*} \mathrm{u}[4]^{\wedge} 8^{*} \mathrm{y} 1^{*} \mathrm{y} 2^{*} \mathrm{x} 1-$ $152719723886674529603520^{*} \mathrm{u}[4]^{\wedge} 5^{*} \mathrm{x} 1^{*} \mathrm{y} 2^{*} \mathrm{x} 2+260969816368722240000^{*} \mathrm{u}[4]^{\wedge} 2^{*} \mathrm{y} 1{ }^{*} \mathrm{y} 2^{*} \mathrm{x} 1$ - 782909449106166720000*u[4]^ $3^{*} \mathrm{y} 1^{*} \mathrm{y} 2^{*} \mathrm{x} 2-264748635488702047680^{*} \mathrm{x} 2^{*} \mathrm{u}[4]^{\wedge} 8^{*} \mathrm{y} 1^{*} \mathrm{x} 1+$ $301732966876507230539520^{*} \mathrm{x} 1^{*} \mathrm{u}[4]^{\wedge} 6^{*} \mathrm{y} 1^{*} \mathrm{x} 2+$

$150862704619133635462080^{*} \mathrm{u}[4]^{\wedge} 7^{*} \mathrm{x} 1{ }^{*} \mathrm{y} 2{ }^{*} \mathrm{x} 2-782909449106166720000 * \mathrm{u}[4]^{\wedge} 5^{*} \mathrm{y} 1{ }^{*} \mathrm{y} 2{ }^{*} \mathrm{x} 2-$ $152980693703043251843520^{*} \mathrm{u}[4]^{\wedge} 3^{*} \mathrm{x} 1{ }^{*} \mathrm{y} 2^{*} \mathrm{x} 2-260969816368722240000^{*} \mathrm{u}[4]^{*} \mathrm{y} 1{ }^{*} \mathrm{y} 2{ }^{*} \mathrm{x} 2-$ $302254906509244675019520^{*} \mathrm{x} 1^{*} \mathrm{u}[4]^{\wedge} 2^{*} \mathrm{y} 1{ }^{*} \mathrm{x} 2-10515855737033206560000^{*} \mathrm{u}[4]^{\wedge} 3^{*} \mathrm{y} 3^{\wedge} 2^{*} \mathrm{x} 0$ - $3505285245677735520000^{*} \mathrm{u}[4]^{*} \mathrm{y} 3^{\wedge} 2^{*} \mathrm{x} 0-373245604005545553686328^{*} \mathrm{x} 1^{*} \mathrm{u}[4]^{\wedge} 2^{*} \mathrm{x} 2{ }^{\wedge} 2$ $27050816406346276574676^{*} \mathrm{x} 0^{\wedge} 2^{*} \mathrm{u}[4]^{\wedge} 8^{*} \mathrm{x} 2-541190092105429129382574^{*} \mathrm{x} 0^{\wedge} 2^{*} \mathrm{u}[4]^{\wedge} 5^{*} \mathrm{x} 1-$ $757426237736436526533012^{*} \mathrm{x} 0 \wedge 2^{*} \mathrm{u}[4]^{\wedge} 6^{*} \mathrm{x} 2+$ $254265981995376158935680^{*} \mathrm{x} 0^{\wedge} 2^{*} \mathrm{u}[4]^{\wedge} 4^{*} \mathrm{x} 2+$ $824463282215282680684692^{*} \mathrm{u}[4]^{\wedge} 2^{*} \mathrm{x} 0^{\wedge} 2^{*} \mathrm{x} 2-288368242652265959004696^{*} \mathrm{u}[4]^{\wedge} 3^{*} \mathrm{x} 22^{\wedge} 3+$ $522372408997503260791116^{*} \mathrm{u}[4]^{\wedge} 6^{*} \mathrm{x} 3^{\wedge} 3-139247580631085788370400^{*} \mathrm{u}[4]^{\wedge} 4^{*} \mathrm{x} 3^{\wedge} 3+$ $1530117962386752815331078^{*} \mathrm{x} 0 \wedge 2^{*} \mathrm{x} 3^{*} \mathrm{u}[4]^{\wedge} 2+$ $144344370709761744313767^{*} \times 0^{\wedge} 2^{*} \mathrm{u}[4]^{\wedge} 8^{*} \mathrm{x} 3+$ $612261595819585411727412^{*} \mathrm{x} 0^{*} \mathrm{u}[4]^{\wedge} 7^{*} \mathrm{x} 3^{\wedge} 2-$ $1815267407791036834597788^{*} \mathrm{x} 0^{*} \mathrm{u}[4]^{\wedge} 5^{*} \mathrm{x} 3^{\wedge} 2$ - 
$1807839190645151843774988^{*} \mathrm{x} 00^{*} \mathrm{u}[4]^{\wedge} 3^{*} \mathrm{x} 3^{\wedge} 2-686498218896452210506476^{*} \mathrm{u}[4]^{\wedge} 2^{*} \mathrm{x} 3^{\wedge} 3-$ $82198370111755628009952^{*} \mathrm{x} 0^{\wedge} 3^{*} \mathrm{u}[4]^{\wedge} 8+23400136985728511842200^{*} \mathrm{u}[4]^{\wedge} 8^{*} \mathrm{x} 0^{*} \mathrm{y} 3^{*} \mathrm{x} 1+$ $8215102967936878166400^{*} \mathrm{u}[4]^{\wedge} 6^{*} \mathrm{y} 0^{*} \mathrm{y} 3^{*} \mathrm{x} 1+2404172946124378166400^{*} \mathrm{u}[4]^{\wedge} 7^{*} \mathrm{y} 0^{*} \mathrm{y} 3^{*} \mathrm{x} 2-$ $1703378537844060916800^{*} \mathrm{u}[4]^{\wedge} 8^{*} \mathrm{y} 0{ }^{*} \mathrm{y} 3^{*} \mathrm{x} 1-2675800712310773299200 * \mathrm{u}[4]^{\wedge} 4{ }^{*} \mathrm{x} 0{ }^{*} \mathrm{y} 3{ }^{*} \mathrm{x} 1+$ $159847042021133214078000^{*} \mathrm{u}[4]^{\wedge} 5^{*} \mathrm{x} 00^{*} \mathrm{y} 3^{*} \mathrm{x} 2-8215102967936878166400^{*} \mathrm{u}[4]^{\wedge} 2^{*} \mathrm{y} 0{ }^{*} \mathrm{y} 3^{*} \mathrm{x} 1$ $-16031201248876865500800^{*} \mathrm{u}[4]^{\wedge} 3^{*} \mathrm{y} 0^{*} \mathrm{y} 3^{*} \mathrm{x} 2-127376920810495699200^{*} \mathrm{x} 3^{*} \mathrm{u}[4]^{\wedge} 4^{*} \mathrm{y} 0{ }^{*} \mathrm{x} 1$ $+211520690079359128164000^{*} \mathrm{x} 3^{*} \mathrm{u}[4]^{\wedge} 5^{*} \mathrm{y} 0 * \mathrm{x} 2-$ $104155857391237840537200^{*} \mathrm{x} 0 * \mathrm{u}[4]^{\wedge} 6^{*} \mathrm{y} 3^{*} \mathrm{x} 1+$ $86456451780590003053200^{*} \mathrm{u}[4]^{\wedge} 7^{*} \mathrm{x} 3^{*} \mathrm{y} 3^{*} \mathrm{x} 1+5815771820536792780800^{*} \mathrm{u}[4]^{\wedge} 8^{*} \mathrm{x} 3^{*} \mathrm{y} 3^{*} \mathrm{x} 2$ - 50246060336957052723600*u[4]^ $3^{*} \mathrm{x} 3^{*} \mathrm{y} 3^{*} \mathrm{x} 1$ $13386706054339337740800^{*} \mathrm{u}[4]^{\wedge} 4^{*} \mathrm{x} 3^{*} \mathrm{y} 3^{*} \mathrm{x} 2-64189092864970693789200{ }^{*} \mathrm{x} 0 * \mathrm{u}[4]^{\wedge} 7^{*} \mathrm{y} 0{ }^{*} \mathrm{x} 1$ $+5252665183180681291200^{*} \mathrm{x} 0^{*} \mathrm{u}[4]^{\wedge} 8^{*} \mathrm{y} 0^{*} \mathrm{x} 2+$ $116526197451077536035600^{*} \mathrm{x} 0 * \mathrm{u}[4]^{\wedge} 3^{*} \mathrm{y} 0^{*} \mathrm{x} 1+$ $12864766421601893260800^{*} \mathrm{x} 0 * \mathrm{u}[4]^{\wedge} 4^{*} \mathrm{y} 0 * \mathrm{x} 2+$ $113101916722552067238000^{*} \mathrm{x} 0 * \mathrm{u}[4]^{\wedge} 2^{*} \mathrm{y} 3^{*} \mathrm{x} 1-$ $119893826198937983299200^{*} \mathrm{u}[4]^{\wedge} 6 * \mathrm{x} 3{ }^{*} \mathrm{y} 0 * \mathrm{x} 1-$ $31698088870094396776800^{*} \mathrm{u}[4]^{\wedge} 7^{*} \mathrm{x} 3^{*} \mathrm{y} 0{ }^{*} \mathrm{x} 2-49347375521419747738800^{*} \mathrm{u}[4]^{\wedge} 7^{*} \mathrm{x} 0{ }^{*} \mathrm{y} 3^{*} \mathrm{x} 2$ $-16031201248876865500800^{*} \mathrm{u}[4]^{\wedge} 5 * \mathrm{y} 0 * \mathrm{y} 3{ }^{*} \mathrm{x} 2+$

$174310678615259216228400^{*} \mathrm{u}[4]^{\wedge} 3^{*} \mathrm{x} 0{ }^{*} \mathrm{y} 3{ }^{*} \mathrm{x} 2+2404172946124378166400^{*} \mathrm{u}[4]^{*} \mathrm{y} 0{ }^{*} \mathrm{y} 3{ }^{*} \mathrm{x} 2-$ $1246569258237651878400^{*} \mathrm{y} 1^{*} \mathrm{y} 3^{*} \mathrm{u}[4]^{\wedge} 7^{*} \mathrm{x} 2+4487081208037044364800^{*} \mathrm{y} 1^{*} \mathrm{y} 3^{*} \mathrm{u}[4]^{\wedge} 5^{*} \mathrm{x} 2$ $+4487081208037044364800^{*} \mathrm{y} 1{ }^{*} \mathrm{y} 3{ }^{*} \mathrm{u}[4]^{\wedge} 3^{*} \mathrm{x} 2-1246569258237651878400^{*} \mathrm{y} 1{ }^{*} \mathrm{y} 3{ }^{*} \mathrm{u}[4]^{*} \mathrm{x} 2$ $269791910343122918400^{*} \mathrm{u}[4]^{\wedge} 7^{*} \mathrm{y} 2^{*} \mathrm{y} 0{ }^{*} \mathrm{x} 2-9036164713779368755200^{*} \mathrm{u}[4]^{\wedge} 5^{*} \mathrm{y} 2^{*} \mathrm{y} 0{ }^{*} \mathrm{x} 2-$ $9036164713779368755200^{*} \mathrm{u}[4]^{\wedge} 3^{*} \mathrm{y} 2 * \mathrm{y} 0 * \mathrm{x} 2-3235493335334612601600^{*} \mathrm{y} 1{ }^{*} \mathrm{u}[4]^{\wedge} 7^{*} \mathrm{y} 0 * \mathrm{x} 1-$ $1479691023253837804800^{*} \mathrm{y} 1^{*} \mathrm{u}[4]^{\wedge} 3^{*} \mathrm{y} 0{ }^{*} \mathrm{x} 1+589398044823556300800^{*} \mathrm{u}[4]^{\wedge} 8^{*} \mathrm{y} 2{ }^{*} \mathrm{y} 3{ }^{*} \mathrm{x} 2+$ $5292190581022112601600^{*} \mathrm{u}[4]^{\wedge} 6^{*} \mathrm{y} 2^{*} \mathrm{y} 3^{*} \mathrm{x} 2-4711635870444400402624^{*} \mathrm{u}[4]^{\wedge} 8^{*} \mathrm{x} 3^{\wedge} 2^{*} \mathrm{x} 2+$ $262970274875361113229774^{*} \mathrm{u}[4]^{\wedge} 5^{*} \mathrm{x} 3^{\wedge} 2^{*} \mathrm{x} 1+$ $873029494601800233756412^{*} \mathrm{u}[4]^{\wedge} 6^{*} \times 3^{\wedge} 2^{*} \mathrm{x} 2+$ $102455159130395102380320^{*} \mathrm{u}[4]^{\wedge} 4^{*} \mathrm{x} 3^{\wedge} 2^{*} \mathrm{x} 2-583345397954875126592092^{*} \mathrm{u}[4]^{\wedge} 2^{*} \mathrm{x} 3{ }^{\wedge} 2^{*} \mathrm{x} 2$ $+310661288725103305069822^{*} \mathrm{x} 0^{\wedge} 2^{*} \mathrm{u}[4]^{\wedge} 7^{*} \mathrm{x} 1-$

$255926340644569072212414^{*} \mathrm{x} 0^{\wedge} 2^{*} \mathrm{u}[4]^{\wedge} 3^{*} \mathrm{x} 1+408696102669433357455982^{*} \mathrm{u}[4]^{*} \mathrm{x} 0{ }^{\wedge} 2^{*} \mathrm{x} 1-$ $463366478560127474353422^{*} \mathrm{u}[4]^{\wedge} 7^{*} \mathrm{x} 3^{\wedge} 2^{*} \mathrm{x} 1+$

$156067093977386686717614^{*} \mathrm{u}[4]^{\wedge} 3^{*} \mathrm{x} 3^{\wedge} 2^{*} \mathrm{x} 1-383040721941571896081582^{*} \mathrm{u}[4]^{*} \mathrm{x} 3^{\wedge} 2^{*} \mathrm{x} 1+$ $7317784803446010316800^{*} \mathrm{u}[4]^{\wedge} 2^{*} \mathrm{y} 1^{*} \mathrm{y} 2^{*} \mathrm{x} 0-6840407029286969049600^{*} \mathrm{u}[4]^{\wedge} 3^{*} \mathrm{y} 1^{*} \mathrm{y} 2^{*} \mathrm{x} 3+$ $3775203587071902492720^{*} \mathrm{x} 2^{*} \mathrm{u}[4]^{\wedge} 8^{*} \mathrm{y} 1^{*} \mathrm{x} 0-321184643146294659911280^{*} \mathrm{x} 1^{*} \mathrm{u}[4]^{\wedge} 5^{*} \mathrm{y} 1^{*} \mathrm{x} 0$ - $29037934060831034145360^{*} \mathrm{x} 1^{*} \mathrm{u}[4]^{\wedge} 6^{*} \mathrm{y} 1 * \mathrm{x} 3-$

$94707266182229158047360^{*} \mathrm{x} 1{ }^{*} \mathrm{u}[4]^{\wedge} 6^{*} \mathrm{y} 2^{*} \mathrm{x} 0+694324500769059886585092^{*} \mathrm{x} 0{ }^{*} \mathrm{u}[4]^{*} \mathrm{x} 3^{\wedge} 2$ $1656926428383907023028998^{*} \mathrm{x} 0^{\wedge} 2^{*} \mathrm{u}[4]^{\wedge} 6 * \mathrm{x} 3-$

$201443153800743691732800^{*} \mathrm{x} 0^{\wedge} 2^{*} \mathrm{u}[4]^{\wedge} 4^{*} \mathrm{x} 3-36619703769999778453440^{*} \mathrm{u}[4]^{\wedge} 5^{*} \mathrm{y} 3^{*} \mathrm{x} 1^{\wedge} 2$ $+12938601868975943415360^{*} \mathrm{u}[4]^{\wedge} 3^{*} \mathrm{y} 3^{*} \mathrm{x} 1^{\wedge} 2+$

$31690138234341298921920^{*} \mathrm{u}[4]^{\wedge} 7^{*} \mathrm{y} 3^{*} \mathrm{x} 1^{\wedge} 2-139233790608873057012960^{*} \mathrm{u}[4]^{\wedge} 2^{*} \mathrm{y} 2^{*} \mathrm{x} 3^{\wedge} 2$ $+34535483262877052851200^{*} \mathrm{u}[4]^{\wedge} 6^{*} \mathrm{y} 1^{\wedge} 2^{*} \mathrm{x} 0-34535483262877052851200^{*} \mathrm{u}[4]^{\wedge} 2^{*} \mathrm{y} 1^{\wedge} 2^{*} \mathrm{x} 0$ - $16833839702634059788800 * \mathrm{y} 0 * \mathrm{u}[4]^{*} \mathrm{y} 2{ }^{*} \mathrm{x} 1+19453141370888165491200 * \mathrm{u}[4]^{\wedge} 7^{*} \mathrm{y} 3 *^{*} 1^{*} \mathrm{x} 1$ - $10429587301835503526400^{*} \mathrm{u}[4]^{\wedge} 5^{*} \mathrm{y} 3^{*} \mathrm{y} 1^{*} \mathrm{x} 1$ -

$10429587301835503526400^{*} \mathrm{u}[4]^{\wedge} 3^{*} \mathrm{y} 3^{*} \mathrm{y} 1^{*} \mathrm{x} 1+19453141370888165491200^{*} \mathrm{u}[4]^{*} \mathrm{y} 3^{*} \mathrm{y} 1{ }^{*} \mathrm{x} 1$ $+18287492306597820633600 * \mathrm{y} 0 * \mathrm{u}[4]^{\wedge} 5^{*} \mathrm{y} 2 * \mathrm{x} 1+$ 
$9615352887762139065600^{*} \mathrm{u}[4]^{\wedge} 8^{*} \mathrm{y} 3^{*} \mathrm{y} 2^{*} \mathrm{x} 1-15163799931725721868800^{*} \mathrm{u}[4]^{\wedge} 6^{*} \mathrm{y} 3^{*} \mathrm{y} 2^{*} \mathrm{x} 1$ $+15163799931725721868800^{*} \mathrm{u}[4]^{\wedge} 2^{*} \mathrm{y} 3^{*} \mathrm{y} 2{ }^{*} \mathrm{x} 1+$ $34885287063860855952960 * \mathrm{u}[4]^{\wedge} 2{ }^{*} \mathrm{y} 3{ }^{*} \mathrm{x} 2{ }^{*} \mathrm{x} 1-33842638385321484922560{ }^{*} \mathrm{y} 0{ }^{*} \mathrm{u}[4]^{*} \mathrm{x} 2{ }^{*} \mathrm{x} 1-$ $4011396122726982313920^{*} \mathrm{u}[4]^{\wedge} 7^{*} \mathrm{y} 3^{*} \mathrm{x} 2^{\wedge} 2+100788637480309390357440^{*} \mathrm{u}[4]^{\wedge} 5^{*} \mathrm{y} 3^{*} \mathrm{x} 2^{\wedge} 2$ $+32363039216800330568640^{*} \mathrm{u}[4]^{\wedge} 3^{*} \mathrm{y} 3^{*} \mathrm{x} 2^{\wedge} 2-$ $516699724916807494890408^{*} \mathrm{x} 1^{*} \mathrm{u}[4]^{\wedge} 7^{*} \mathrm{x} 0 * \mathrm{x} 3-$ $1600568824640666316452664^{*} \mathrm{x} 1^{*} \mathrm{u}[4]^{\wedge} 5^{*} \mathrm{x} 0 * \mathrm{x} 3-$ $931956840553106760734904 * \mathrm{x} 1 * \mathrm{u}[4]^{\wedge} 3^{*} \mathrm{x} 0 * \mathrm{x} 3+$ $228905006250211834745964^{*} \mathrm{u}[4]^{\wedge} 8^{*} \mathrm{x} 2^{*} \mathrm{x} 0^{*} \mathrm{x} 3+$ $570149239879579833330168 * \mathrm{u}[4]^{\wedge} 6{ }^{*} \mathrm{x} 2 * \mathrm{x} 0 * \mathrm{x} 3+$ $566620423059128183124480^{*} \mathrm{u}[4]^{\wedge} 4^{*} \mathrm{x} 2 * \mathrm{x} 0 * \mathrm{x} 3$ $765066573314150568029688^{*} \mathrm{u}[4]^{\wedge} 2^{*} \mathrm{x} 2{ }^{*} \mathrm{x} 0{ }^{*} \mathrm{x} 3-176159006252981653322160{ }^{*} \mathrm{y} 1{ }^{*} \mathrm{u}[4]{ }^{*} \mathrm{x} 3{ }^{\wedge} 2$ $+183150478774606845757680^{*} \mathrm{u}[4]^{*} \mathrm{y} 2^{*} \mathrm{x} 00^{*} \mathrm{x} 3-609625497322946856996648^{*} \mathrm{x} 0^{*} \mathrm{u}[4]^{*} \mathrm{x} 3^{*} \mathrm{x} 1$ $+398298800585482588627224^{*} \mathrm{x} 3^{\wedge} 2^{*} \mathrm{u}[4]^{*} \mathrm{x} 2+2182121811398056320000^{*} \mathrm{u}[4]^{*} \mathrm{y} 1^{\wedge} 2^{*} \mathrm{x} 2-$ $304010708818397443200^{*} \mathrm{y} 0^{*} \mathrm{u}[4]^{*} \mathrm{y} 2^{*} \mathrm{x} 3+390873809080778284966104^{*} \mathrm{u}[4]^{\wedge} 7^{*} \mathrm{x} 2^{*} \mathrm{x} 3{ }^{\wedge} 2-$ $453794530922377054782384^{*} \mathrm{x} 1^{*} \mathrm{u}[4]^{\wedge} 6^{*} \mathrm{x} 0^{\wedge} 2-223631302071428595011232^{*} \mathrm{x} 1^{*} \mathrm{u}[4]^{\wedge} 8^{*} \mathrm{x} 0^{\wedge} 2$ $+31611853833646616347632^{*} \mathrm{x} 1^{*} \mathrm{u}[4]^{\wedge} 8^{*} \mathrm{x} 3^{\wedge} 2+$ $175534456114016122133760^{*} \mathrm{x} 1^{*} \mathrm{u}[4]^{\wedge} 4^{*} \mathrm{x} 0^{\wedge} 2+33197272474252600151184^{*} \mathrm{x} 1^{*} \mathrm{u}[4]^{\wedge} 6^{*} \mathrm{x} 3^{\wedge} 2$ $+248560108789543718004144^{*} \mathrm{x} 1^{*} \mathrm{u}[4]^{\wedge} 2^{*} \mathrm{x} 0^{\wedge} 2-$ $209767316443979342021760^{*} \mathrm{x} 1^{*} \mathrm{u}[4]^{\wedge} 4^{*} \mathrm{x} 3^{\wedge} 2-36459467568276005921832^{*} \mathrm{u}[4]^{\wedge} 5^{*} \mathrm{x} 2^{*} \mathrm{x} 0^{\wedge} 2-$ $15393127687705132610904^{*} \mathrm{u}[4]^{\wedge} 7^{*} \mathrm{x} 2{ }^{*} \mathrm{x} 0{ }^{\wedge} 2-22769073044702239181040{ }^{*} \mathrm{x} 1{ }^{*} \mathrm{u}[4]^{*} \mathrm{y} 2{ }^{*} \mathrm{x} 3-$ $269791910343122918400^{*} \mathrm{y} 0{ }^{*} \mathrm{u}[4]^{*} \mathrm{y} 2^{*} \mathrm{x} 2+3753845339908216860960^{*} \mathrm{y} 1{ }^{*} \mathrm{u}[4]^{\wedge} 8^{*} \mathrm{x} 3{ }^{*} \mathrm{x} 2-$ $105107119833060679847040^{*} \mathrm{u}[4]^{\wedge} 5^{*} \mathrm{y} 2^{*} \mathrm{x} 00^{*} \mathrm{x} 1-5657046035049273335040^{*} \mathrm{u}[4]^{\wedge} 8^{*} \mathrm{y} 2^{*} \mathrm{x} 0{ }^{*} \mathrm{x} 2$ $-171505137962050628388480^{*} \mathrm{u}[4]^{\wedge} 6^{*} \mathrm{y} 2 * \mathrm{x} 0 * \mathrm{x} 2+$ $73864698671534542387200^{*} \mathrm{u}[4]^{\wedge} 4^{*} \mathrm{y} 2^{*} \mathrm{x} 0 * \mathrm{x} 2-407536907154848158550952^{*} \mathrm{u}[4]^{\wedge} 3^{*} \mathrm{x} 2^{*} \mathrm{x} 0 \wedge 2$ - $2114910961636112640000^{*} \mathrm{u}[4]^{\wedge} 2^{*} \mathrm{y} 3^{\wedge} 2^{*} \mathrm{x} 0+6344732884908337920000^{*} \mathrm{y} 0^{\wedge} 2^{*} \mathrm{u}[4]^{\wedge} 5^{*} \mathrm{x} 3+$ $1057455480818056320000^{*} \mathrm{u}[4]^{\wedge} 8^{*} \mathrm{y} 3^{\wedge} 2^{*} \mathrm{x} 0+2114910961636112640000^{*} \mathrm{u}[4]^{\wedge} 6^{*} \mathrm{y} 3^{\wedge} 2^{*} \mathrm{x} 0-$ $12259101840722340495336^{*} \mathrm{u}[4]^{*} \mathrm{x} 2^{\wedge} 3-131127668336196485788758^{*} \mathrm{x} 2^{\wedge} 2^{*} \mathrm{u}[4]^{\wedge} 5^{*} \mathrm{x} 0-$ $66970070972876334606436^{*} \mathrm{x} 22^{\wedge} 2^{*} \mathrm{u}[4]^{\wedge} 6^{*} \mathrm{x} 3-209342780196345260625600^{*} \mathrm{x} 22^{\wedge} 2^{*} \mathrm{u}[4]^{\wedge} 4^{*} \mathrm{x} 3-$ $152244872989893913371164^{*} \mathrm{u}[4]^{\wedge} 2^{*} \mathrm{x} 2^{\wedge} 2^{*} \mathrm{x} 3-92951651785753967238614^{*} \mathrm{x} 1^{\wedge} 2^{*} \mathrm{u}[4]^{\wedge} 7^{*} \mathrm{x} 0-$ $39935313531385166791242^{*} \mathrm{u}[4]^{\wedge} 3^{*} \mathrm{x} 1^{\wedge} 2^{*} \mathrm{x} 0+14187779254024909912186^{*} \mathrm{u}[4]^{*} \mathrm{x} 1^{\wedge} 2^{*} \mathrm{x} 0-$ $42469296085505214694986^{*} \mathrm{u}[4]^{\wedge} 7^{*} \mathrm{x} 2^{\wedge} 2^{*} \mathrm{x} 0-134167533243681763653558^{*} \mathrm{x} 2^{\wedge} 2^{*} \mathrm{u}[4]^{\wedge} 3^{*} \mathrm{x} 0-$ $35636997226565505207786^{*} \mathrm{u}[4]^{*} \mathrm{x} 22^{\wedge} 2^{*} \mathrm{x} 0+6340662535971952849920^{*} \mathrm{x} 0{ }^{*} \mathrm{u}[4]^{\wedge} 8^{*} \mathrm{y} 3^{*} \mathrm{x} 3+$ $54705824621143940201280^{*} \mathrm{y} 2{ }^{*} \mathrm{u}[4]^{*} \mathrm{x} 2{ }^{*} \mathrm{x} 3+41648903971971693927120^{*} \mathrm{u}[4]^{\wedge} 7^{*} \mathrm{x} 2{ }^{*} \mathrm{y} 3{ }^{*} \mathrm{x} 1-$ $35149226098361639153040 * \mathrm{u}[4]^{\wedge} 3^{*} \mathrm{x} 2{ }^{*} \mathrm{y} 3{ }^{*} \mathrm{x} 1+$

$34031092556259059788800^{*} \mathrm{u}[4]^{\wedge} 2^{*} \mathrm{y} 3^{*} \mathrm{y} 1{ }^{*} \mathrm{x} 2+2114910961636112640000^{*} \mathrm{y} 0 * \mathrm{u}[4]^{\wedge} 2^{*} \mathrm{y} 3^{*} \mathrm{x} 3$ $-1057455480818056320000^{*} \mathrm{y} 0^{*} \mathrm{u}[4]^{\wedge} 8^{*} \mathrm{y} 3^{*} \mathrm{x} 3+2114910961636112640000^{*} \mathrm{y} 0^{\wedge} 2^{*} \mathrm{u}[4]^{\wedge} 7^{*} \mathrm{x} 3$ $+6344732884908337920000^{*} \mathrm{y} 0^{\wedge} 2^{*} \mathrm{u}[4]^{\wedge} 3^{*} \mathrm{x} 3+2114910961636112640000^{*} \mathrm{u}[4]^{*} \mathrm{y} 0^{\wedge} 2^{*} \mathrm{x} 3-$ $64834727760974526809760^{*} \mathrm{x} 1^{\wedge} 2^{*} \mathrm{u}[4]^{\wedge} 2^{*} \mathrm{y} 2+$ $224710114777734991514400^{*} \mathrm{x} 3^{*} \mathrm{u}[4]^{\wedge} 3^{*} \mathrm{y} 0^{*} \mathrm{x} 2-$ $144680040392635560880800^{*} \mathrm{u}[4]^{\wedge} 6^{*} \mathrm{x} 3^{*} \mathrm{y} 3^{*} \mathrm{x} 2+$ $142915194381921223140000 * \mathrm{u}[4]^{\wedge} 2^{*} \mathrm{x} 3^{*} \mathrm{y} 3{ }^{*} \mathrm{x} 2+$ $166555944583701786784800^{*} \mathrm{x} 0^{*} \mathrm{u}[4]^{\wedge} 6^{*} \mathrm{y} 0^{*} \mathrm{x} 2-$ $165313038205724893524000^{*} \mathrm{x} 0^{*} \mathrm{u}[4]^{\wedge} 2^{*} \mathrm{y} 0^{*} \mathrm{x} 2-46505598970918745588400^{*} \mathrm{x} 0{ }^{*} \mathrm{u}[4]^{*} \mathrm{y} 3^{*} \mathrm{x} 2-$ $42151118493226749354000^{*} \mathrm{u}[4]^{\wedge} 5^{*} \mathrm{x} 3{ }^{*} \mathrm{y} 3^{*} \mathrm{x} 1+$ 
$108692225423715954906000^{*} \mathrm{x} 0 * \mathrm{u}[4]^{\wedge} 5^{*} \mathrm{y} 0{ }^{*} \mathrm{x} 1+$ $14894046634003371061200 * \mathrm{u}[4]^{\wedge} 8{ }^{*} \mathrm{x} 3{ }^{*} \mathrm{y} 00^{*} \mathrm{x} 1+$ $1218901805527634847651432^{*} \mathrm{u}[4]^{\wedge} 5^{*} \mathrm{x} 2^{*} \mathrm{x} 3^{\wedge} 2-5701689027427826328024^{*} \mathrm{u}[4]^{*} \mathrm{x} 2^{*} \mathrm{x} 00^{\wedge} 2+$ $1607095675279188610224552^{*} \mathrm{u}[4]^{\wedge} 3^{*} \mathrm{x} 2 * \mathrm{x} 3^{\wedge} 2-$ $201738959883937775761920^{*} \mathrm{y} 1^{*} \mathrm{u}[4]^{\wedge} 6^{*} \mathrm{x} 0^{\wedge} 2-264706217793813116160^{*} \mathrm{y} 1^{*} \mathrm{u}[4]^{\wedge} 8^{*} \mathrm{x} 0^{\wedge} 2-$ $8812925434587974919840^{*} \mathrm{y} 1^{*} \mathrm{u}[4]^{\wedge} 8^{*} \mathrm{x} 3^{\wedge} 2+157136156438291463006720^{*} \mathrm{y} 1^{*} \mathrm{u}[4]^{\wedge} 2^{*} \mathrm{x} 00^{\wedge} 2-$ $37590763774646312755200^{*} \mathrm{y} 1^{*} \mathrm{u}[4]^{\wedge} 4^{*} \mathrm{x} 0 \wedge 2+142517711152618368649920^{*} \mathrm{y} 1^{*} \mathrm{u}[4]^{\wedge} 6^{*} \mathrm{x} 3^{\wedge} 2$ - 44541207078465349324800* $1^{*} \mathrm{u}[4]^{\wedge} 4^{*} \mathrm{x} 3^{\wedge} 2-82087018804328884067520^{*} \mathrm{u}[4]^{\wedge} 7^{*} \mathrm{y} 2^{*} \mathrm{x} 0^{\wedge} 2$ $+107442415347022061040960^{*} \mathrm{y} 1^{*} \mathrm{u}[4]^{\wedge} 7^{*} \mathrm{x} 0^{*} \mathrm{x} 3-$ $402101184633382271200320 * \mathrm{y} 1 * \mathrm{u}[4]^{\wedge} 5 * \mathrm{x} 0 * \mathrm{x} 3-$ $389860913392722752915520^{*} \mathrm{y}^{*} \mathrm{u}[4]^{\wedge} 3^{*} \mathrm{x} 0 * \mathrm{x} 3+$ $36178817214587586484320^{*} \mathrm{u}[4]^{\wedge} 8^{*} \mathrm{y} 2^{*} \mathrm{x} 0{ }^{*} \mathrm{x} 3+5110425547932466257600^{*} \mathrm{u}[4]^{\wedge} 8^{*} \mathrm{y} 3^{*} \mathrm{x} 2^{*} \mathrm{x} 0$ $+8204524479548535686400^{*} \mathrm{y} 0 * \mathrm{u}[4]^{\wedge} 8^{*} \mathrm{x} 1^{*} \mathrm{x} 0+521506444419501465600^{*} \mathrm{y} 0 * \mathrm{u}[4]^{\wedge} 4^{*} \mathrm{x} 1^{*} \mathrm{x} 0$ $+229955038619269017600^{*} \mathrm{y} 0^{*} \mathrm{u}[4]^{*} \mathrm{y} 2^{*} \mathrm{x} 0+22301401722823156377600^{*} \mathrm{u}[4]^{\wedge} 7^{*} \mathrm{y} 3^{*} \mathrm{y} 1^{*} \mathrm{x} 0$ $+59892165497469469132800^{*} \mathrm{u}[4]^{\wedge} 5^{*} \mathrm{y} 3^{*} \mathrm{y} 1 * \mathrm{x} 0+$ $1952166772778787302400^{*} \mathrm{u}[4]^{\wedge} 7^{*} \mathrm{y} 3^{*} \mathrm{y} 2^{*} \mathrm{x} 3-1155539352663638092800^{*} \mathrm{u}[4]^{\wedge} 5^{*} \mathrm{y} 3^{*} \mathrm{y} 2{ }^{*} \mathrm{x} 3-$ $1155539352663638092800^{*} \mathrm{u}[4]^{\wedge} 3^{*} \mathrm{y} 3^{*} \mathrm{y} 2^{*} \mathrm{x} 3-9664020690168327854400^{*} \mathrm{u}[4]^{\wedge} 7^{*} \mathrm{y} 3^{*} \mathrm{x} 2{ }^{*} \mathrm{x} 3+$ $21346904889750088601280^{*} \mathrm{x} 1^{\wedge} 2^{*} \mathrm{u}[4]^{*} \mathrm{y} 2-2182121811398056320000^{*} \mathrm{u}[4]^{\wedge} 2^{*} \mathrm{y} 1^{*} \mathrm{y} 2^{*} \mathrm{x} 2-$ $19453141370888165491200^{*} \mathrm{u}[4]^{*} \mathrm{y} 1{ }^{\wedge} 2^{*} \mathrm{x} 3-1952166772778787302400^{*} \mathrm{u}[4]^{*} \mathrm{y} 3^{\wedge} 2^{*} \mathrm{x} 2$; 